\title{
Mass spectrometric investigation of pharmaceuticals in environmental matrices: homogenate analysis
}

\author{
Townsend, Rachel
}

How to cite:

Townsend, Rachel (2019) Mass spectrometric investigation of pharmaceuticals in environmental matrices: homogenate analysis. Doctoral thesis, Swansea University.

http://cronfa.swan.ac.uk/Record/cronfa50205

Use policy:

This item is brought to you by Swansea University. Any person downloading material is agreeing to abide by the terms of the repository licence: copies of full text items may be used or reproduced in any format or medium, without prior permission for personal research or study, educational or non-commercial purposes only. The copyright for any work remains with the original author unless otherwise specified. The full-text must not be sold in any format or medium without the formal permission of the copyright holder. Permission for multiple reproductions should be obtained from the original author.

Authors are personally responsible for adhering to copyright and publisher restrictions when uploading content to the repository.

Please link to the metadata record in the Swansea University repository, Cronfa (link given in the citation reference above.)

http://www.swansea.ac.uk/library/researchsupport/ris-support/ 


\title{
Mass spectrometric investigation of pharmaceuticals in environmental matrices: homogenate analysis.
}

\author{
Submitted to Swansea University in fulfilment \\ of the requirements for the Degree of \\ Doctor of Philosophy
}

By

Rachel Townsend

SWANSEA UNIVERSITY

2019 


\section{Summary}

In the last ten years there has been considerable focus on the impact of pollution on the environment driven by research and government policies. With particular interest in soils and environmental waters there is the need to monitor for a wide range of potential organic pollutants, including pesticides, personal care products and pharmaceuticals. The research focus has shifted to the study of wastewater, which has been largely un-investigated as an environmental matrix, with an aim to detect lower amounts than those achieved with current methods. Current approaches for analysing complex environmental matrices such as soil and wastewater effluent are typically multi-step analyses using a range of procedures and apparatus, resulting in methods that are time and resource consuming, unsuitable for highthroughput analysis. This study has investigated new approaches to monitoring concentrations of commonly used pharmaceuticals and biocides in environmental samples, as detailed by UK Water Industry Research and the Chemical Investigation Programme (CIP). A modified QuEChERS sample preparation method has been developed and tested for the extraction of a selection of pharmaceuticals of interest to CIP and extended to biocides, as newly proposed pollutants following an initial investigation in sludge. These were analysed, with results showing sixteen target analytes of variable lipophilicity/acidity were successfully extracted using the developed protocol. Excellent repeatability within a "control" sample of soil was achieved with a relative standard deviation of $<10 \%$ for the majority of pharmaceuticals and $<15 \%$ for the biocides, with low matrix effects, and recovery values of between 40-75\%. This method was applied to two samples of locally sourced treated sludgecake, two samples of homogenised biota (mussel tissue) and a sample of locally sourced treated effluent as part of a qualitative and quantitation study. A selection of pharmaceuticals and the suite of biocides were quantifiable within each sample matrix. This novel sample preparation method is labour-saving and cost effective, offering an improved approach for multiple sample matrices for high throughput analysis versus current protocols. 


\section{Declarations}

\section{DECLARATION}

This work has not previously been accepted in substance for any degree and is not being concurrently submitted in candidature for any degree.

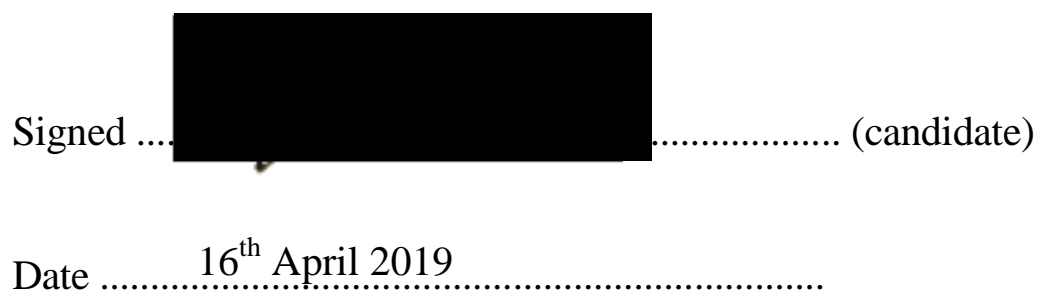

\section{STATEMENT 1}

This thesis is the result of my own investigations, except where otherwise stated. Where correction services have been used, the extent and nature of the correction is clearly marked in a footnote(s). Other sources are acknowledged by footnotes giving explicit references. A bibliography is appended.

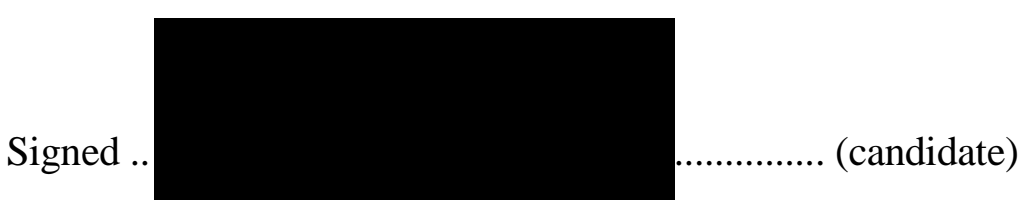

Date $16^{\text {th }}$ April 2019

\section{STATEMENT 2}

I hereby give consent for my thesis, if accepted, to be available for photocopying and for interlibrary loan, and for the title and summary to be made available to outside organisations.

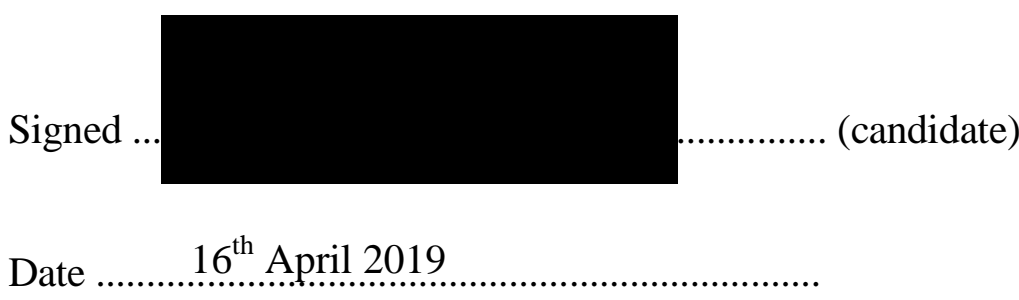




\section{Contents Page}

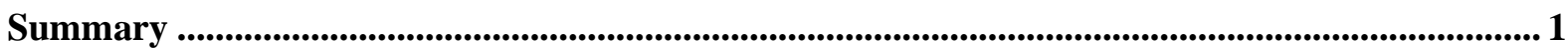

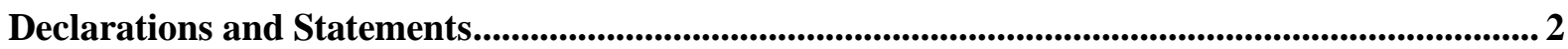

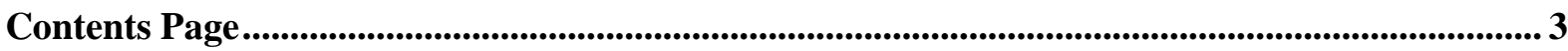

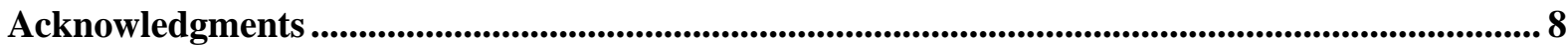

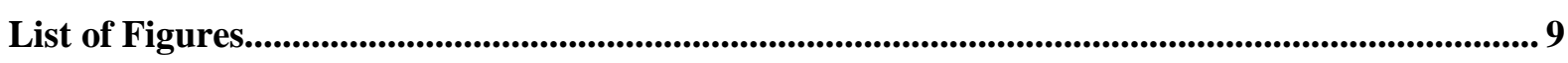

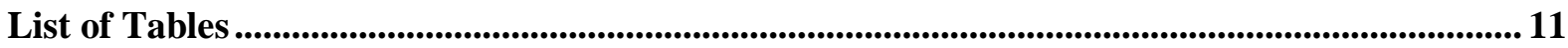

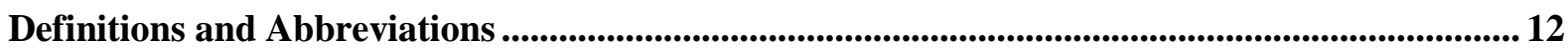

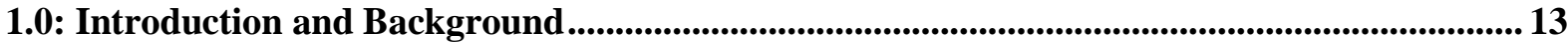

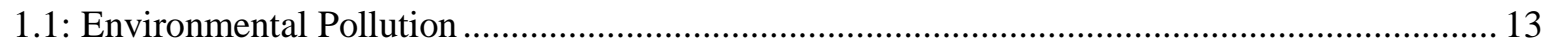

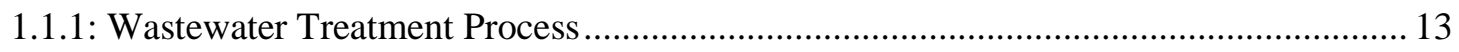

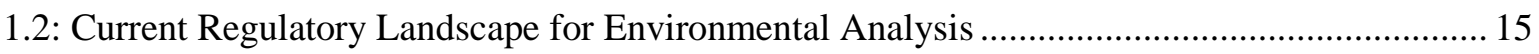

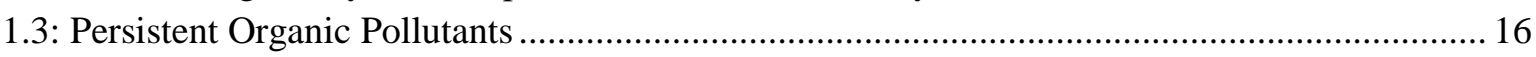

1.3.1: Pharmaceuticals as Persistent Organic Pollutants in the Environment .......................... 17

1.3.2: Biocides as Persistent Organic Pollutants in the Environment ...................................... 18

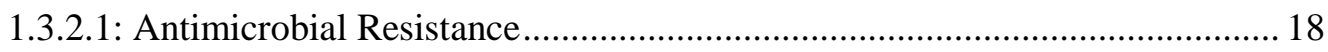

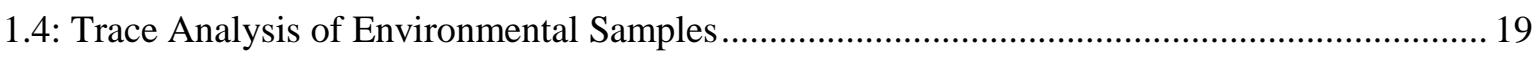

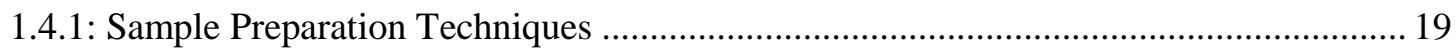

1.4.1.1: Solid-Phase Extraction .................................................................................. 19

1.4.1.2: Quick, Easy, Cheap, Effective, Rugged and Safe (QuEChERS).................. 21

1.4.2: Liquid Chromatography........................................................................................ 22

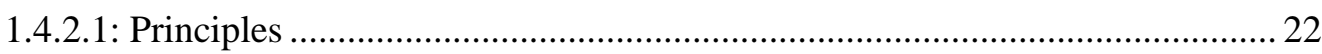

1.4.2.2: Chromatographic Parameters................................................................... 23

1.4.2.3: Column Characteristics for Reversed-Phase Chromatography...................... 25

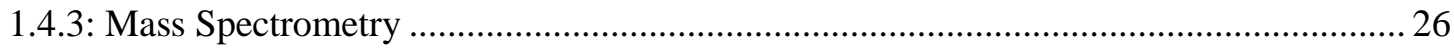

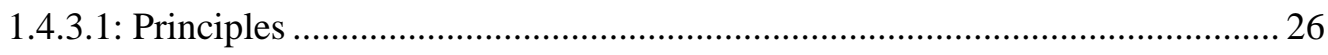

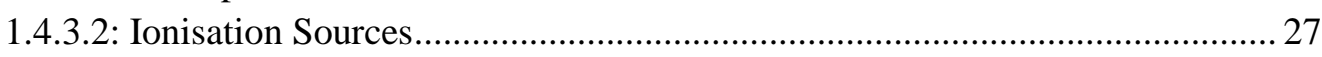

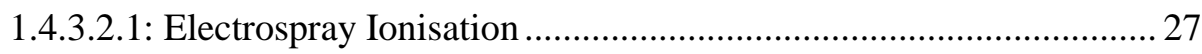

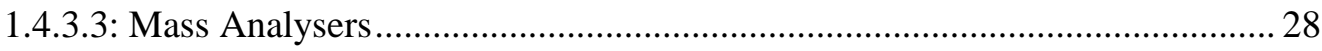

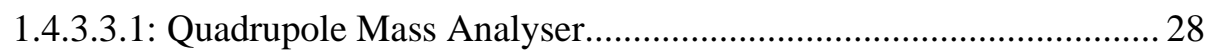

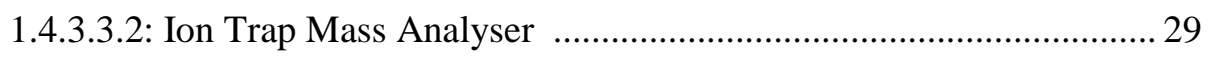

1.4.3.3.3: Orbitrap Mass Analyser ................................................................. 30

1.4.3.3.4: Quadrupole Time-of-Flight Mass Analyser ................................. 31

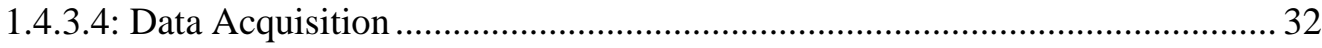

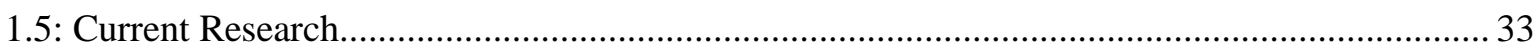

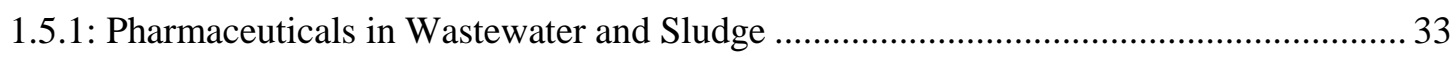

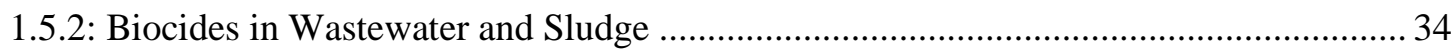

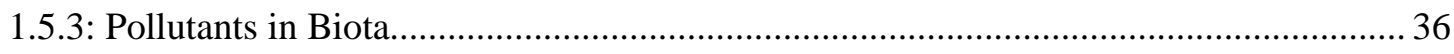

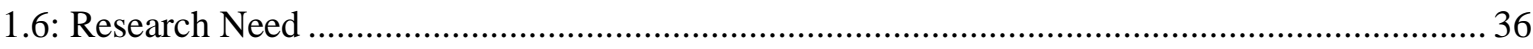




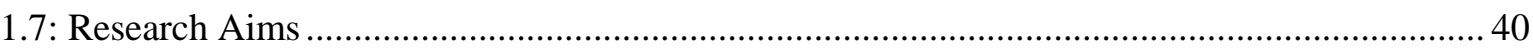

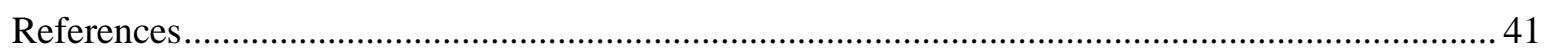

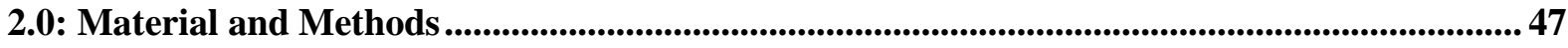

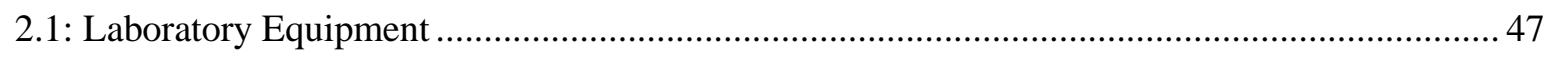

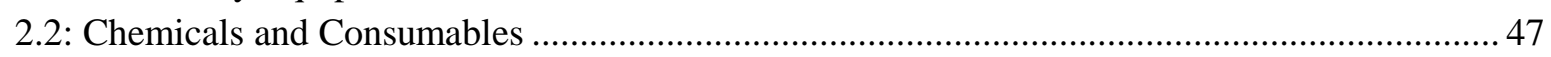

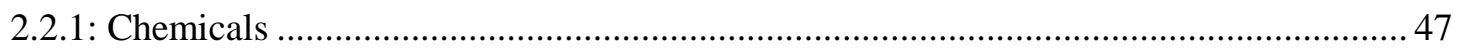

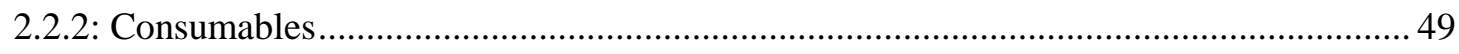

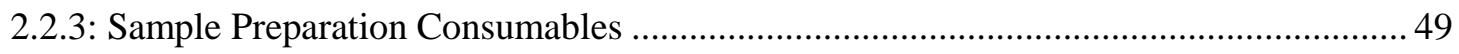

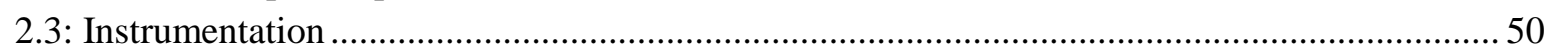

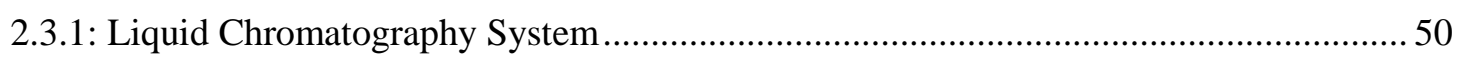

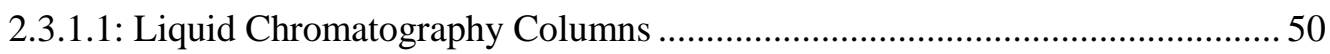

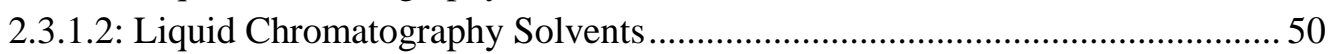

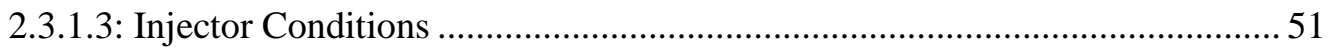

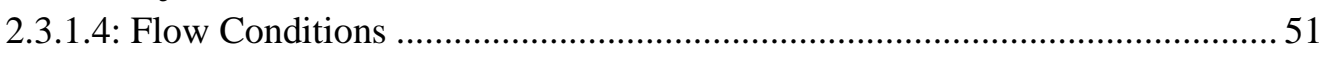

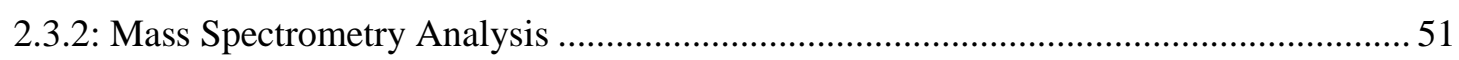

2.3.2.1: Quantitation Method (ZQ4000) .......................................................... 52

2.3.2.2: Pharmaceutical Screen and Biocide Quantitation Method (LCQ) .................. 53

2.3.2.3: Qualitative Screen (LTQ Orbitrap) ................................................................5

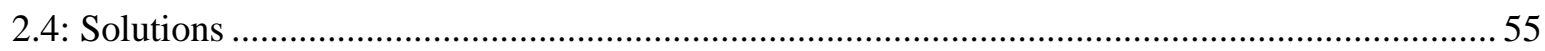

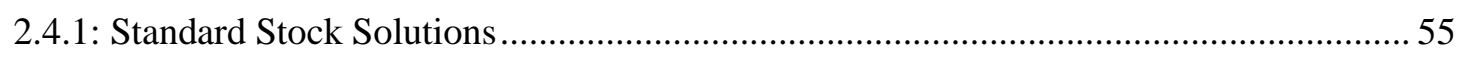

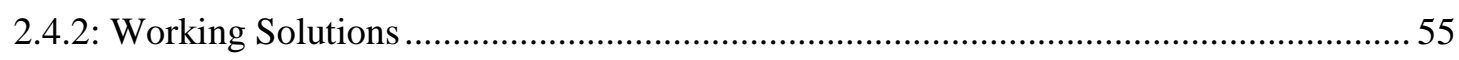

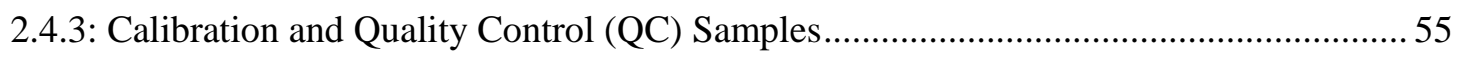

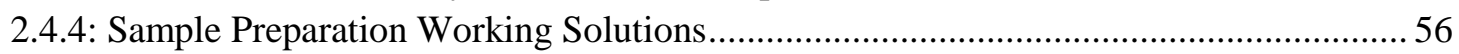

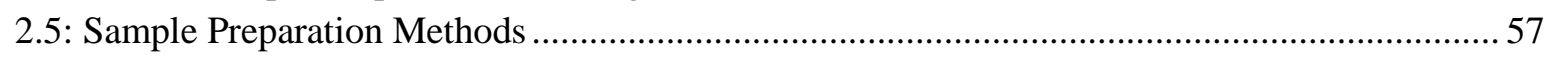

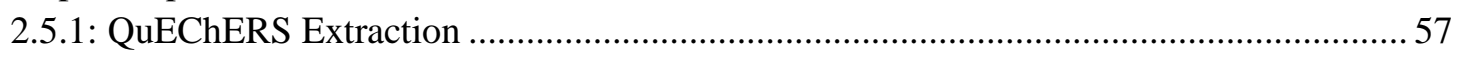

2.5.1.1: Spike Before Extraction Quality Controls ................................................. 58

2.5.1.2: Spike After Extraction Quality Controls ..................................................... 59

2.5.2: Environmental Protection Agency (EPA), Method 1694 Extraction................................ 59

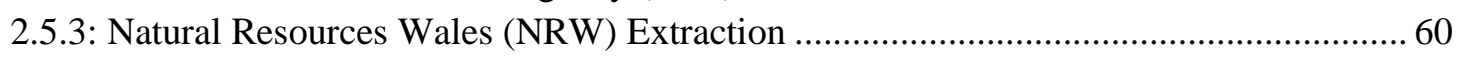

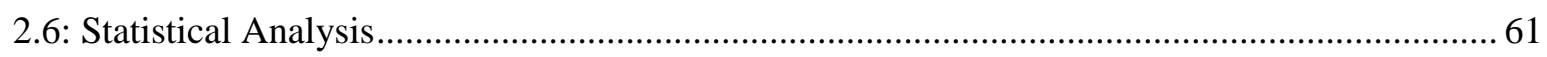

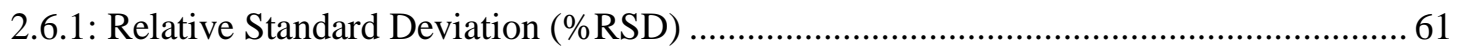

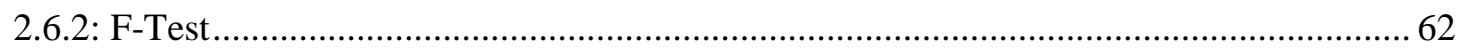

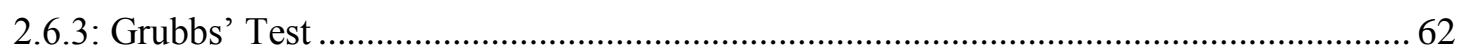

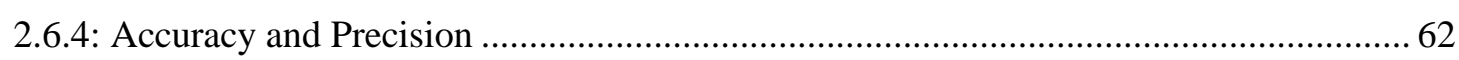

2.6.5: Instrument Detection Limit (IDL) and Instrument Quantitation Limit (IQL) ................ 63

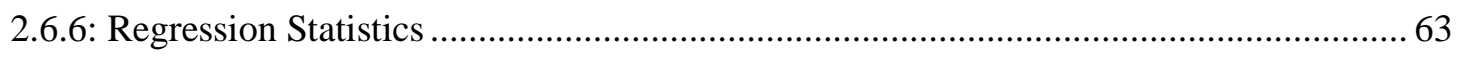

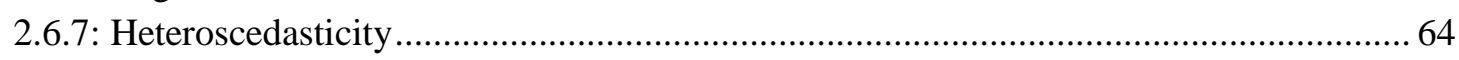

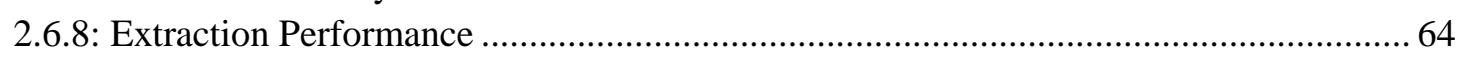

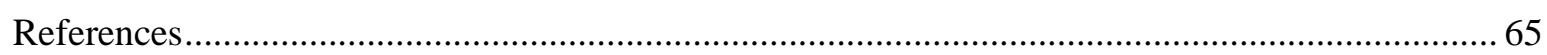

3.0: Liquid Chromatography-Mass Spectrometry Method Development........................................66

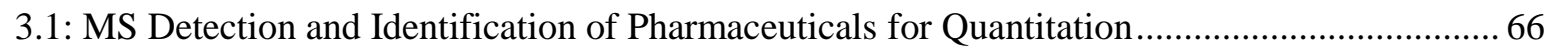

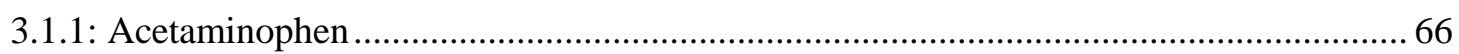

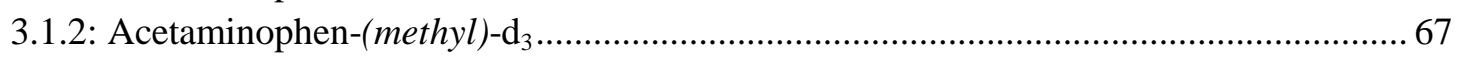

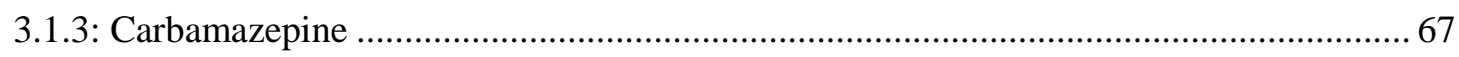

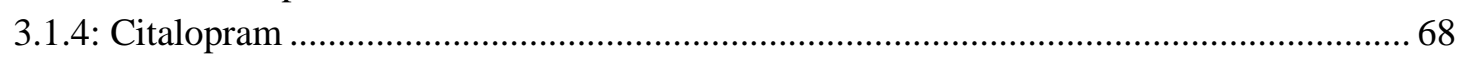

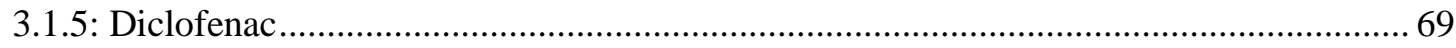




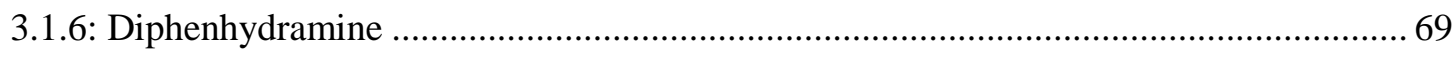

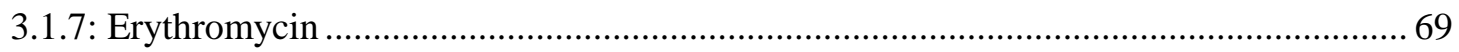

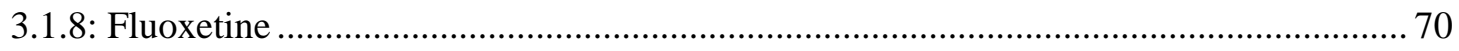

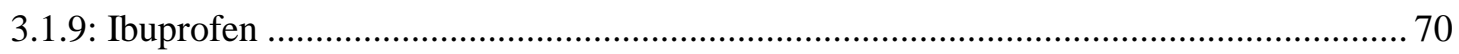

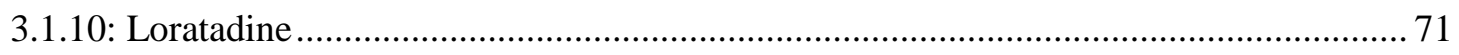

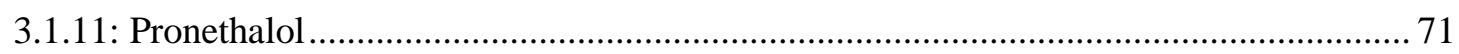

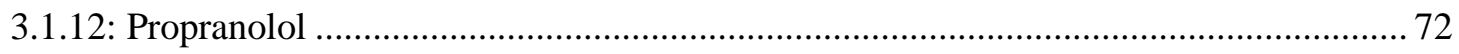

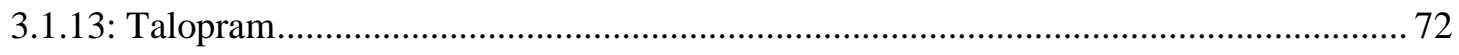

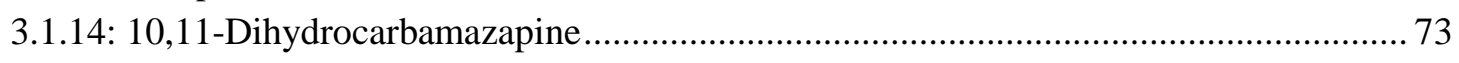

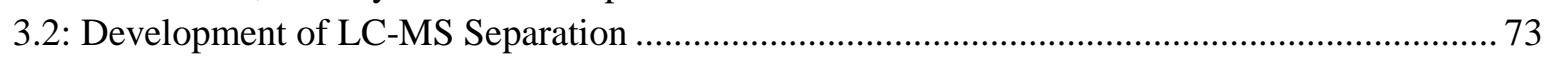

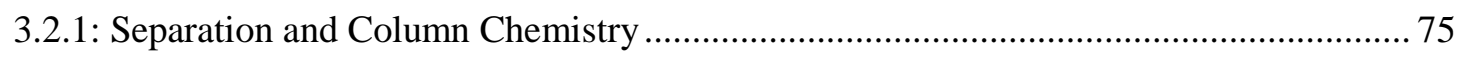

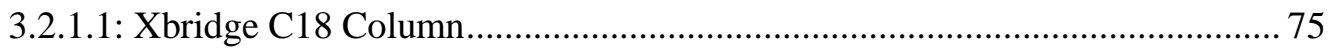

3.2.1.2: Xselect Charged Surface Hybrid (CSH) Column ......................................... 76

3.2.1.3: Xselect High Strength Silica (HSS) T3 Column ......................................... 76

3.2.2: Chromatographic Performance …………….............................................................. 77

3.2.2.1: Comparison of Column Performance ........................................................ 77

3.2.2.1.1: Xbridge C18: Chromatographic Separation Performance .............. 78

3.2.2.1.1.1: Repeatability and Reproducibility ............................... 78

3.2.2.1.2: Xselect HSS T3: Chromatographic Separation Performance ........ 79

3.2.2.1.2.1: Repeatability and Reproducibility ............................... 80

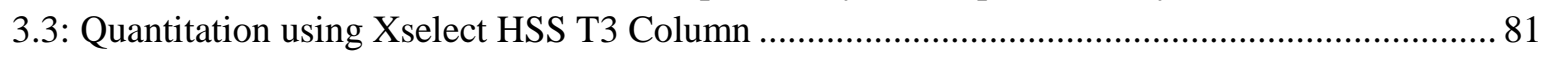

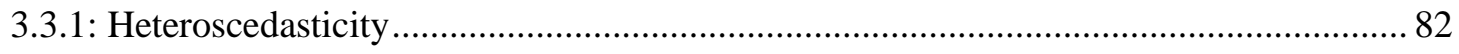

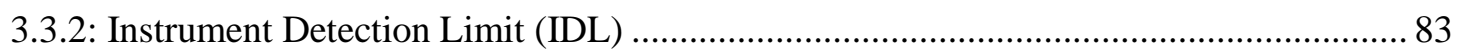

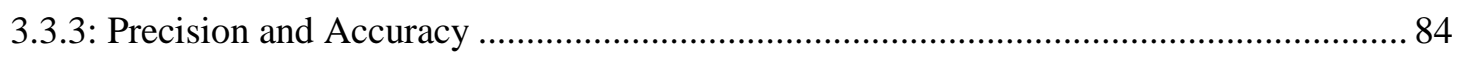

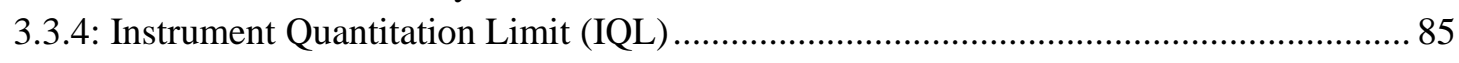

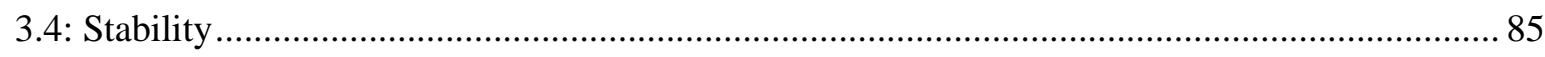

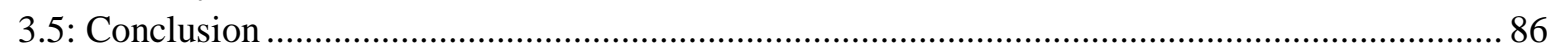

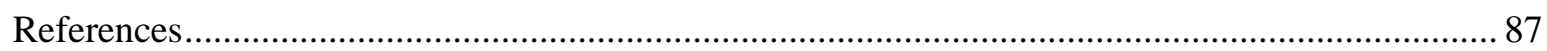

4.0: Liquid Chromatography-Mass Spectrometry Method Development: Biocides ...................... 89

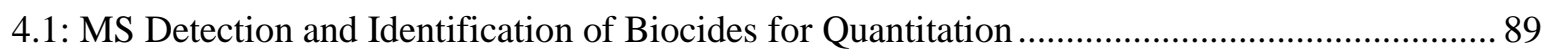

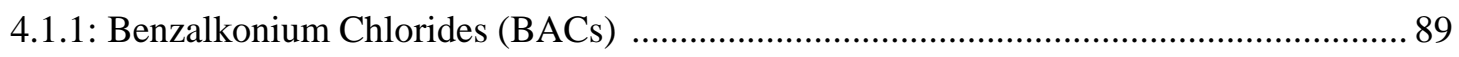

4.1.2: Didecyldimethylammonium Bromide (DDMA) ……............................................... 90

4.1.3: Hexadecyltrimethylammonium Chloride (HDTMA) ................................................ 90

4.2: Semi-Quantitative Method Development for Biocides............................................................. 91

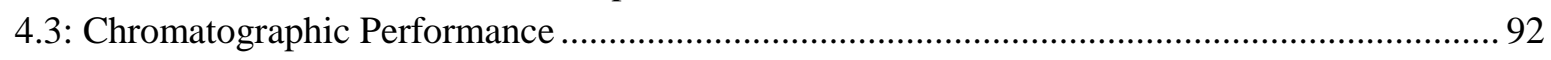

4.3.1: Chromatographic Repeatability and Reproducibility ………..................................... 93

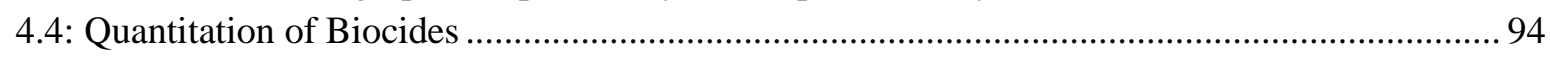

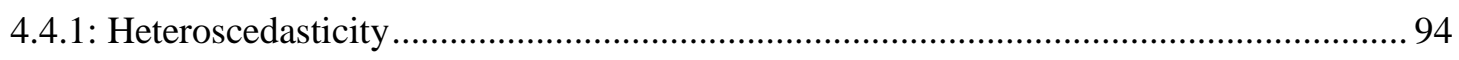

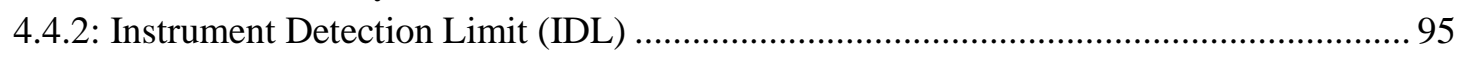

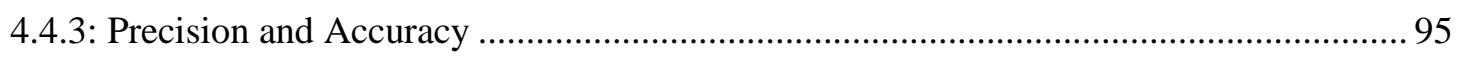

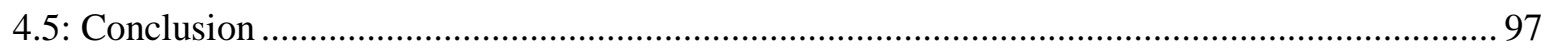

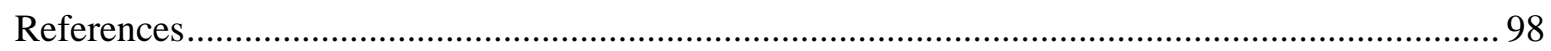

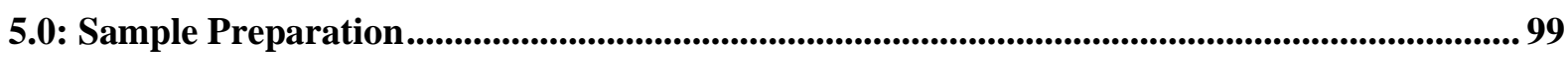

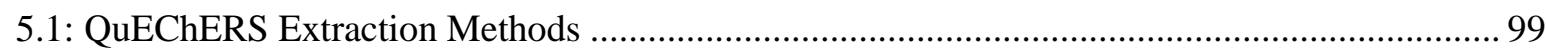

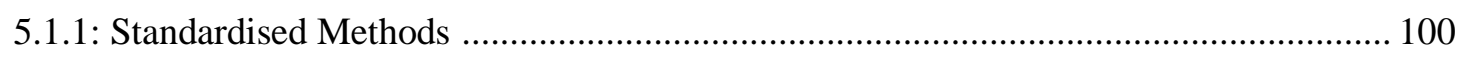

5.1.1.1: Unbuffered QuEChERS Extractions ..................................................... 100 


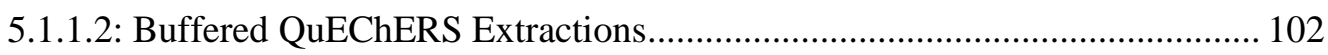

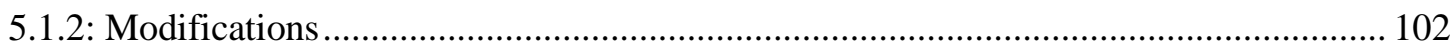

5.1.2.1: Evaluation of Initial Extraction Kit .......................................................... 102

5.1.2.1.1: Mix and Match Approach to Extraction ....................................... 103

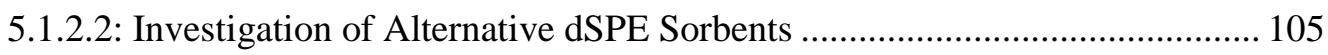

5.1.2.3: Optimisation of Custom QuEChERS Extraction........................................ 106

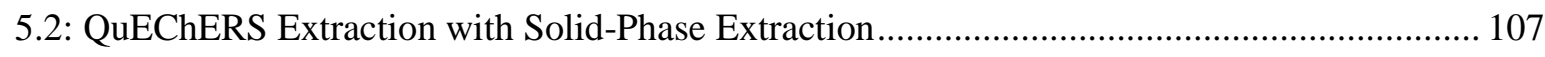

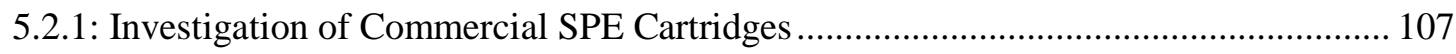

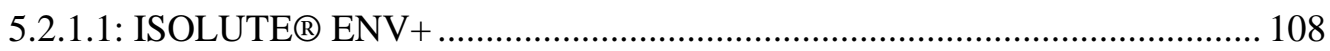

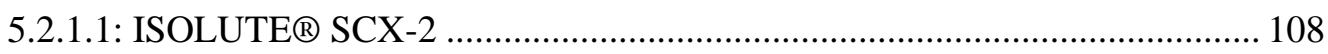

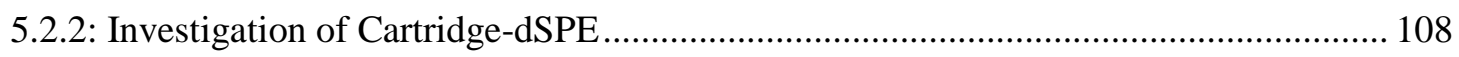

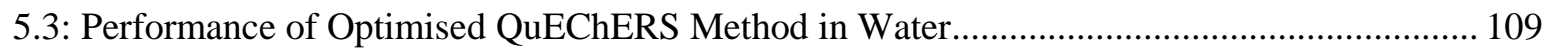

5.4: Performance of Optimised QuEChERS Method in Soil ....................................................... 110

5.4.1: Comparison Study with Industrial Method............................................................... 111

5.5: Application of Optimised QuEChERS Method: Pharmaceuticals and Biocides ...................... 112

5.5.1: Performance of Optimised QuEChERS Method in Water ......................................... 112

5.5.2: Performance of Optimised QuEChERS Method in Soil............................................ 115

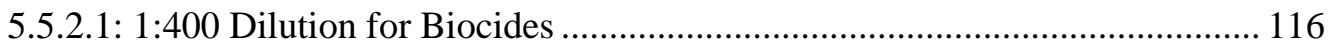

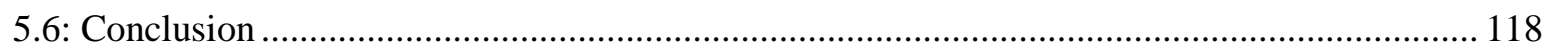

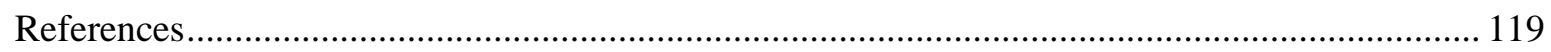

6.0: Method Application: Complex Matrices .......................................................................................... 121

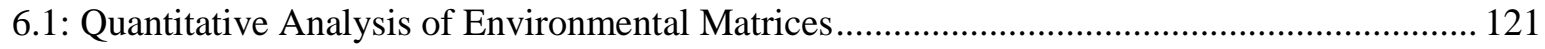

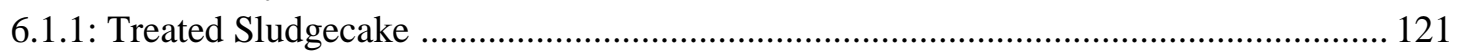

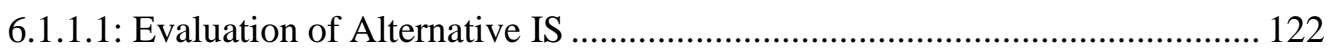

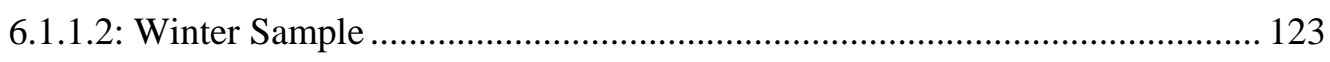

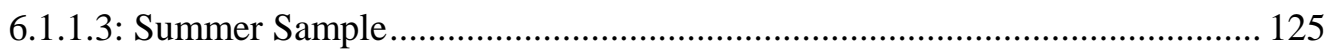

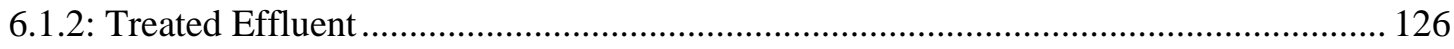

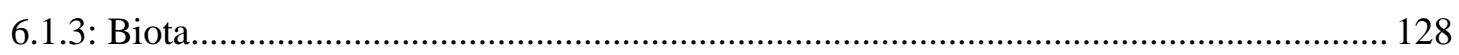

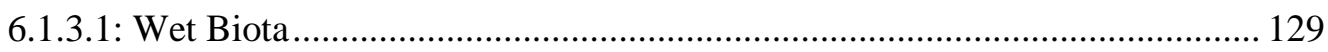

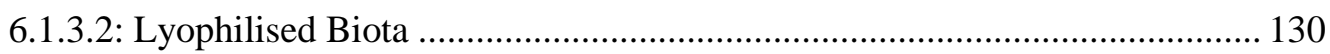

6.1.3.3: Manual Integration of Later Eluting Peaks................................................ 131

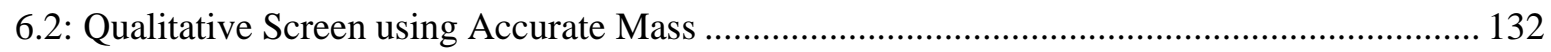

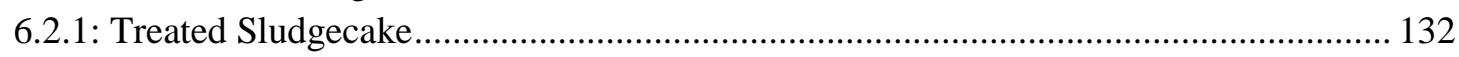

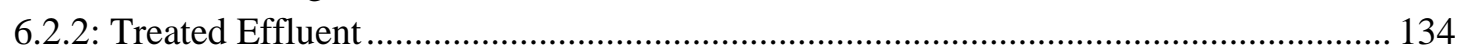

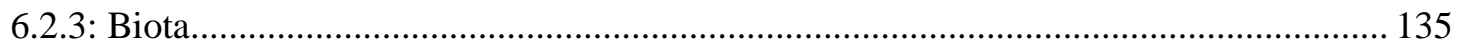

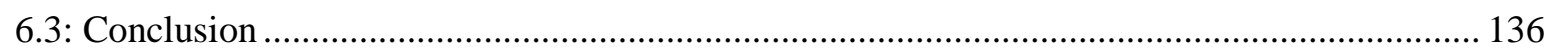

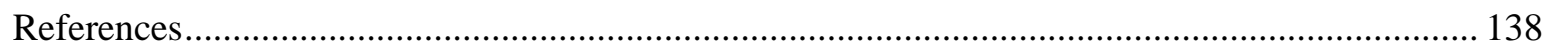

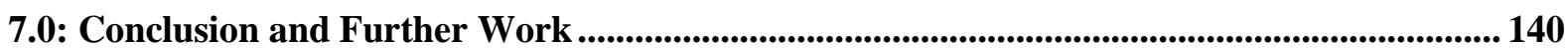

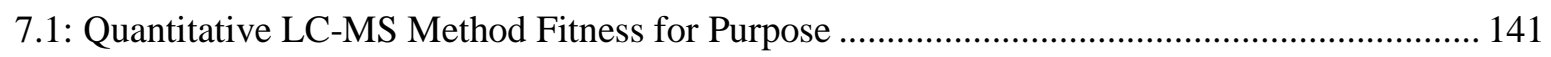

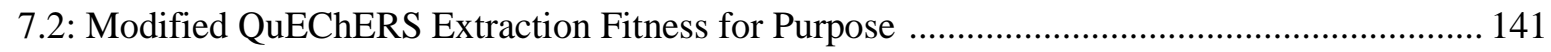

7.3: Qualitative and Quantitative Analysis within Environmental Matrices .................................... 142

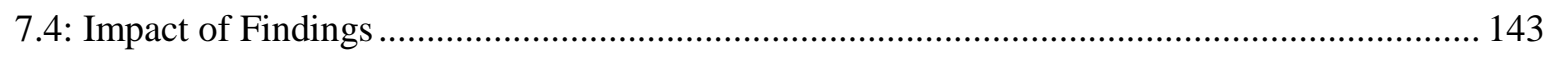

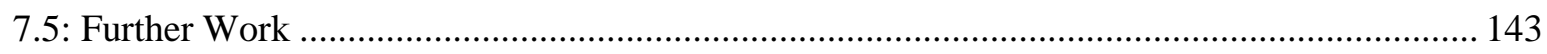

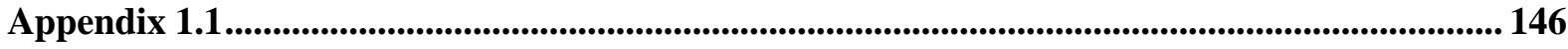




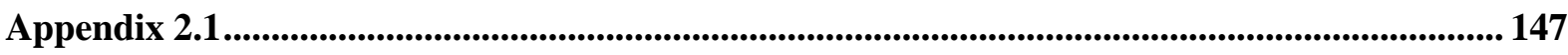

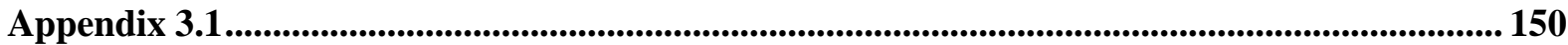

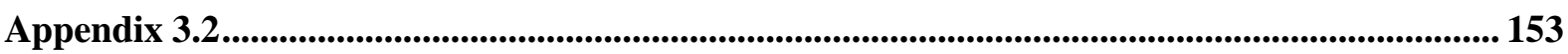

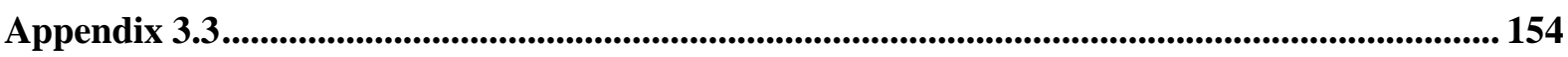

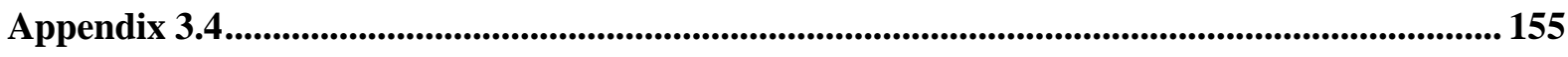

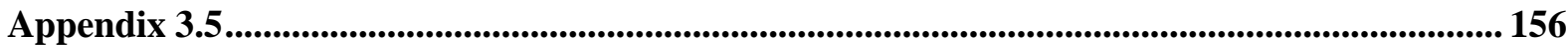

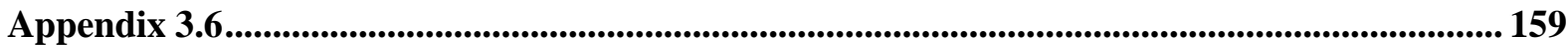

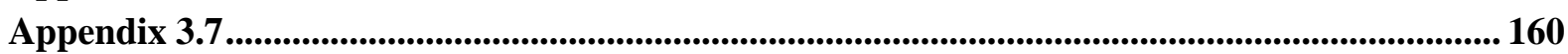

Appendix 4.1 ....................................................................................................................................................... 162

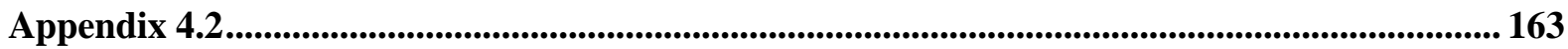

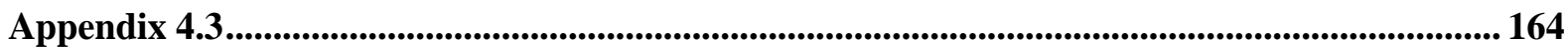

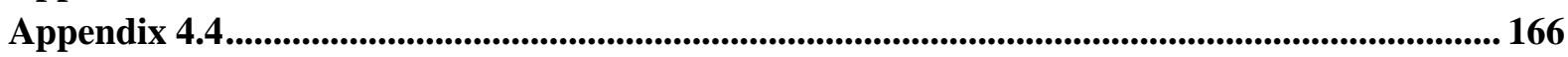

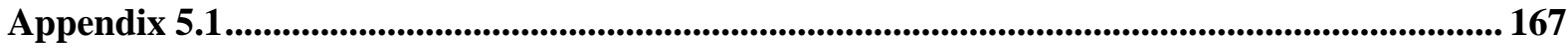

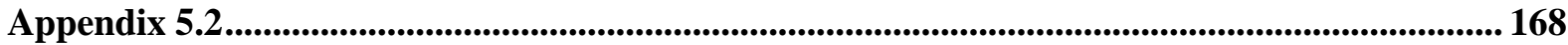

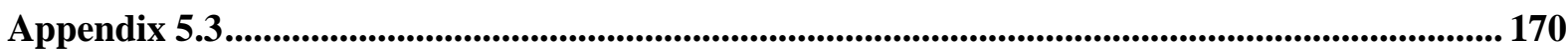

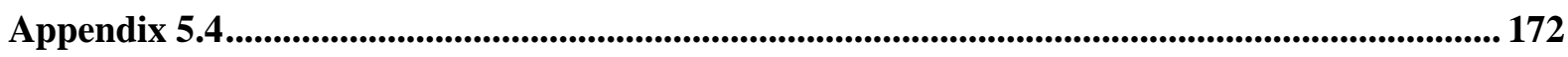

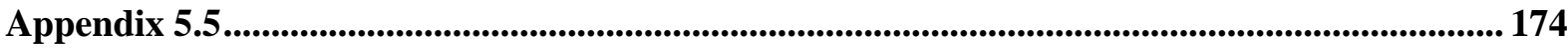

Appendix 6.1

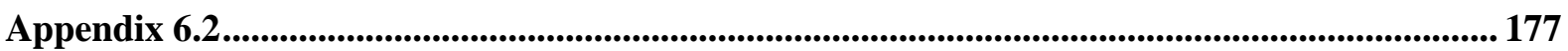




\section{Acknowledgements}

Firstly, I would like to express my sincere gratitude to my project supervisor, Dr Ruth Godfrey, for her continuous and unwavering support of my Ph.D studies. Her patience, motivation, and immense knowledge helped me throughout the entire process and I could not have imagined having a better advisor and mentor.

I would also like to thank the rest of my supervisory team: Professor Gareth Brenton and Dr Geertje van Keulen for their continued encouragement and for their expertise in providing an alternative perspective to some of the numerous challenges I have faced throughout my research. I would also like to thank Dr Claire Desbrow of Biotage for her help with all things sample prep, her knowledge and expertise have been invaluable and I honestly couldn't have done this without her.

My sincere thanks also go to Dr Christopher Williams, Dr Ann Hunter, Dr Mark Wyatt and Miss Maria Moruzzi of the National Mass Spectrometry Facility, who, throughout the years have not only provided me access to their laboratory and research facilities, but also have provided support and encouragement, through good times and bad.

Lastly, I would like to thank my family: my parents, Christopher and Wendy and my sister Claire for all their love and support, and for believing in me, even when I didn't. I'd also like to thank my partner Rob, for his love, encouragement and patience during the last two years. Words cannot wholly express my love and thanks to everyone who has helped me get here today, I will be forever grateful. 


\section{List of Figures}

1.1: $\quad$ Schematic of the Wastewater Treatment Process ................................................................... 14

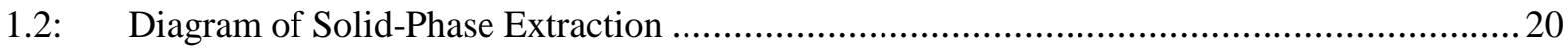

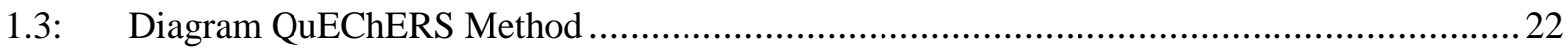

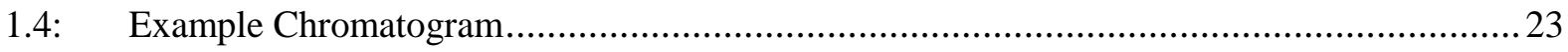

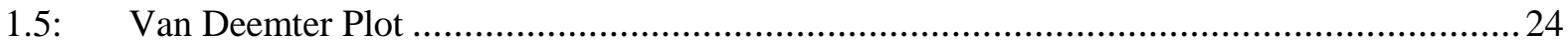

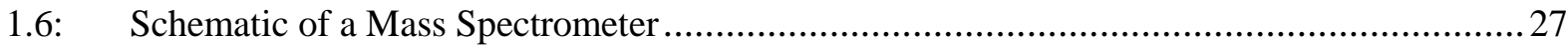

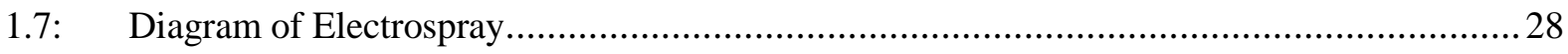

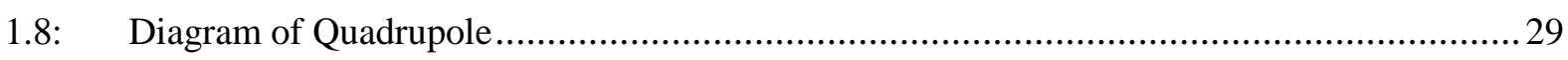

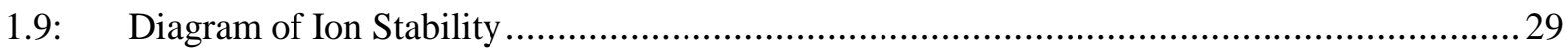

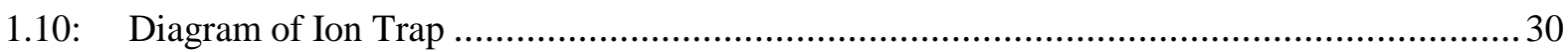

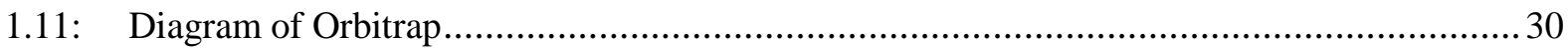

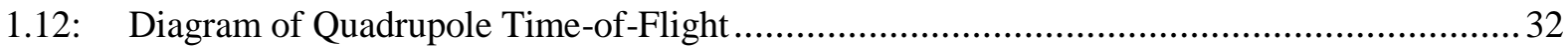

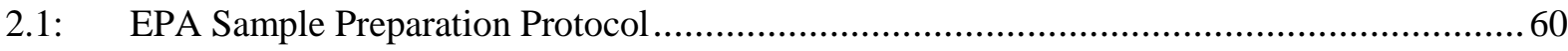

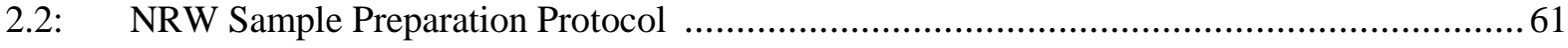

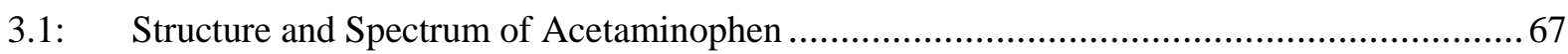

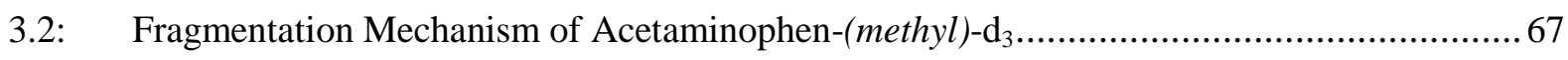

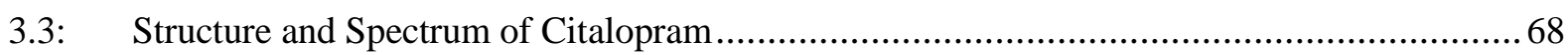

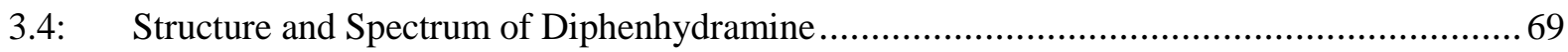

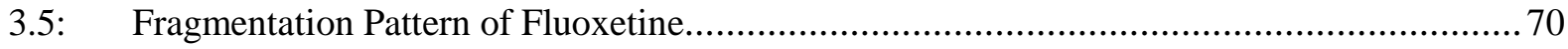

3.6: $\quad$ Structure and Spectrum of Ibuprofen ...................................................................... 71

3.7: $\quad$ Structure and Spectrum of Loratadine …................................................................. 71

3.8: $\quad$ Fragmentation Pattern of Pronethalol …..................................................................... 72

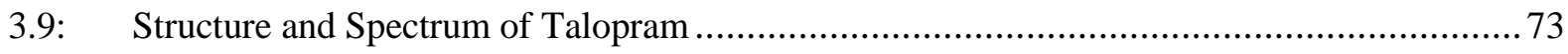

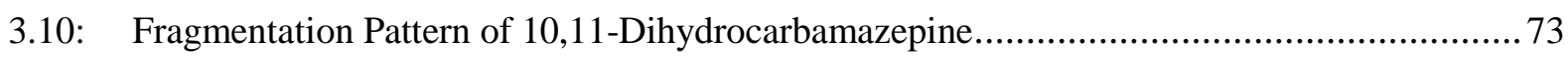

3.11: Chromatograms of Carbamazepine using Xbridge and Xselect HSS T3 Column .................77

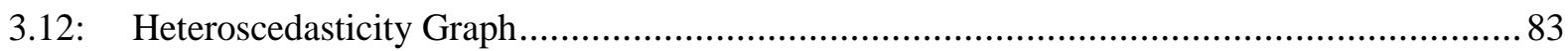

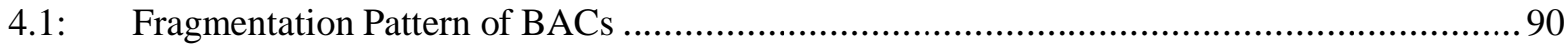

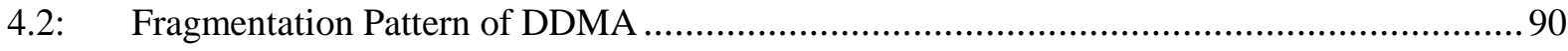

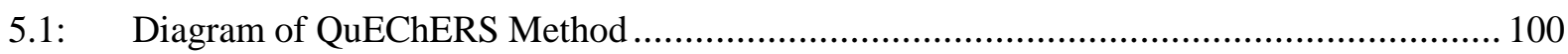

5.2: Chromatograms of Effect of Salt on Propranolol ............................................................ 101

5.3: Chromatograms of Standardised vs. Optimised QuEChERS on Propranolol....................... 104

5.4: Chromatograms of Effect of dSPE on Acetaminophen................................................. 106 
5.5: Graph of Matrix Effects and Recovery of Pharmaceuticals in Water............................... 109

5.6: Graph of Matrix Effects and Recovery of Pharmaceuticals in Soil ................................... 111

5.7: Graph of Matrix Effects and Recovery of Biocides in Water ......................................... 113

5.8: $\quad$ Graph of Matrix Effects and Recovery of Biocides in Water........................................... 114

5.9: $\quad$ Graph of Recovery of Pharmaceuticals with Biocides in Water ...................................... 115

5.10: Graph of Matrix Effects of Pharmaceuticals with Biocides in Soil .................................. 116

5.11: Graph of Matrix Effects and Recovery of Biocides in Soil .......................................... 117

5.12: Graph of Recovery of Pharmaceuticals with Biocides in Soil ....................................... 117

6.1: Graph of Percentage Change in Concentration within Winter and Summer Sludge ............ 126

6.2: Chromatograms for Acetaminophen-(methyl) $-\mathrm{d}_{3}$ in Standard and Wet Biota .................... 127

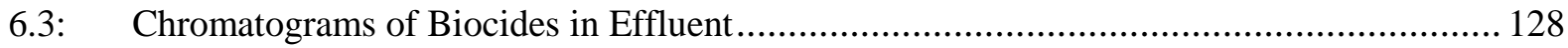

6.4: Chromatograms of Extracted Sludgecake, Wet and Lyophilised Biota ............................. 129

6.5: Extracted Ion Chromatograms and Spectra for Pharmaceuticals in Wet Biota .................. 130

6.6: Extracted Ion Chromatograms and Spectra for Pharmaceuticals in Lyophilised Biota ........ 131

6.7: Qualitative Screen: Peaks of Interest in Effluent............................................................. 134

6.8: Qualitative Screen: Peaks of Interest in Biota.................................................................. 135 


\section{List of Tables}

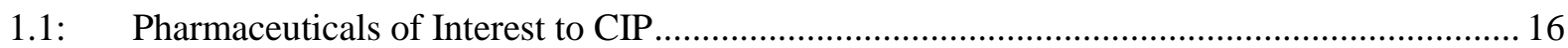

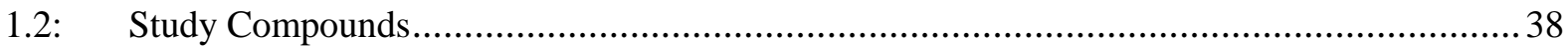

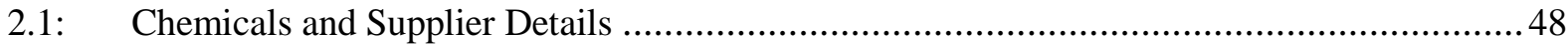

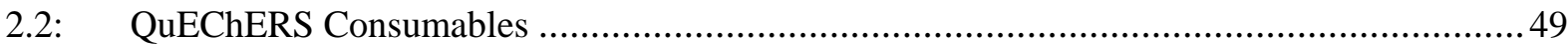

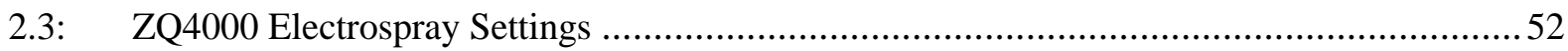

2.4: $\quad$ Optimised Cone Voltages for each Pharmaceutical.........................................................53

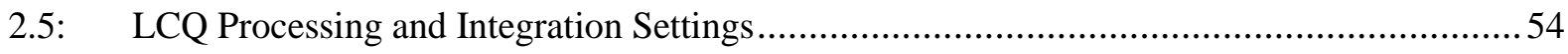

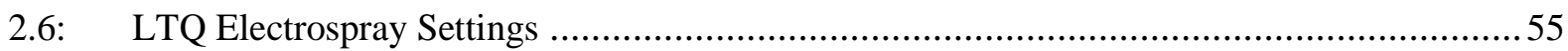

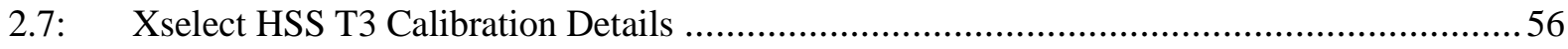

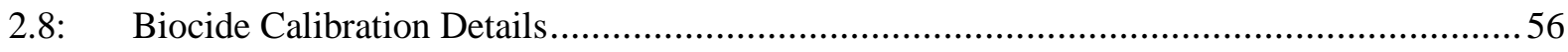

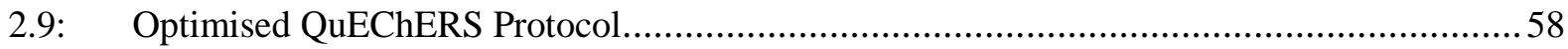

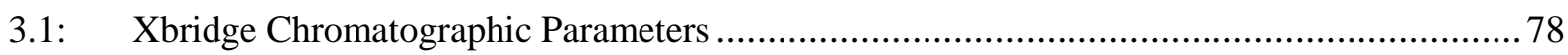

3.2: Xbridge Retention Time Reproducibility for Pharmaceuticals .......................................... 79

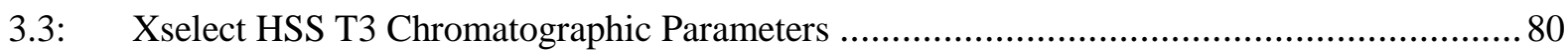

3.4: Xselect HSS T3 Injection Repeatability for Pharmaceuticals......................................... 81

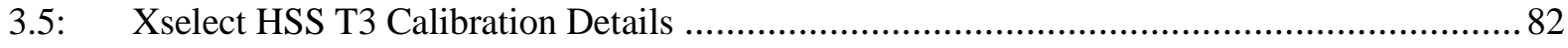

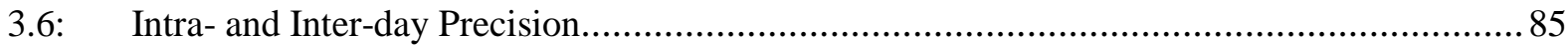

4.1: $\quad$ Mass Spectrometer Parameters for QAC Quantitation ................................................... 92

4.2: Chromatographic Parameters for Biocides ................................................................ 93

4.3: $\quad$ Retention Time Reproducibility for Biocides ............................................................... 93

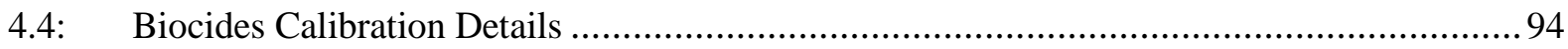

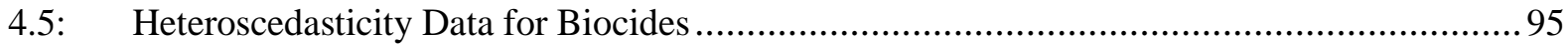

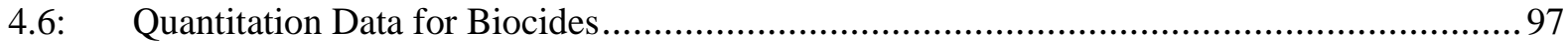

6.1: Quantitation Data for Pharmaceuticals using Alternative Internal Standards...................... 123

6.2: Identification of Pharmaceuticals in Winter Sludge Sample ......................................... 124

6.3: Identification of Biocides in Winter Sludge Sample .................................................... 124

6.4: Identification of Pharmaceuticals in Summer Sludge Sample ........................................ 125

6.5: Identification of Biocides in Summer Sludge Sample ................................................... 126

6.6: Concentrations of Pharmaceuticals in Biota - Manual Integration ................................... 132

6.7: Potential Identification of Peaks of Interest in Sludge Samples....................................... 133

6.8: Potential Identification of Peaks of Interest in Effluent .............................................. 135

6.9: Potential Identification of Peaks of Interest in Biota ..................................................... 136 


\section{Definitions and Abbreviations}

$\begin{array}{clcl}\text { 10,11-DHC } & \text { 10,11-Dihydrocarbamazepine } & m / z & \text { Mass-to-charge ratio } \\ {\text { Acet- }{ }_{3}} & \text { Acetaminophen-(methyl)-d } \mathrm{d}_{3} & \text { MS } & \text { Mass spectrometry } \\ \text { BAC } & \text { Benzalkonium chloride } & \text { PA } & \text { Peak area } \\ \text { CE } & \text { Collision energy } & \text { PCP } & \text { Personal care products } \\ \text { CID } & \text { Collision induced dissociation } & \text { POP } & \text { Persistent organic pollutant } \\ \text { CIP } & \text { Chemical Investigation Programme } & \text { PSA } & \text { Primary secondary amine } \\ \text { CV } & \text { Cone voltage } & \text { QAC } & \text { Quaternary ammonium compound } \\ \text { Da } & \text { Dalton } & \text { QC } & \text { Quality control } \\ \text { DDMA } & \text { Dialkyldimethylammonium chloride } & \text { RRF } & \text { Relative response factor } \\ \text { dSPE } & \text { Dispersive solid-phase extraction } & \text { SAE } & \text { Spike after extraction } \\ \text { EPA } & \text { Environmental Protection Agency } & \text { SBE } & \text { Spike before extraction } \\ \text { EQS } & \text { Environmental Quality Standards } & \text { SIM } & \text { Selected ion monitoring } \\ \text { ESI } & \text { Electrospray ionisation } & \text { SPE } & \text { Solid-phase extraction } \\ \text { EU } & \text { European Union } & \text { SRM } & \text { Selected reaction monitoring } \\ \text { FDA } & \text { Food and Drug Administration } & \text { WFD } & \text { Water Framework Directive } \\ \text { GCD } & \text { Graphitized carbon black } & \% M E & \text { Percentage matrix effects } \\ \text { HDTMA } & \text { Hexadecyltrimethylammonium chloride } & \% \text { RE } & \text { Percentage recovery } \\ \text { IDL } & \text { Instrument detection limit } & \% R E C & \text { Percentage relative error } \\ \text { IQL } & \text { Instrument quantitation limit } & \% R S D & \text { Percentage relative standard deviation } \\ \text { IS } & \text { Internal standard } & & \\ \text { LC } & \text { Liquid chromatography } & & \end{array}$




\section{Chapter 1: Introduction and Background}

In the last twenty years, there has been considerable focus on the impact of pollution on the environment as a result of research and government policies. With particular interest in soils and environmental waters is the need to monitor for a wide range of potential organic pollutants, including pesticides, personal care products (PCP) and pharmaceuticals. For the latter, the research focus has shifted to the study of wastewater, which has been largely uninvestigated as an environmental matrix [1], and the need to detect lower trace concentrations than those achieved with current methods used in the environmental industry $[2,3]$. This study aims to investigate a new method of analysis, suitable for quantifying trace concentrations of compounds of interest to the Chemical Investigation Programme, in environmental wastewater and biota samples, as detailed by current EU regulations.

\section{1: Environmental Pollution}

Environmental pollution is the contamination of the environment by the introduction of any substance (solid, liquid or gas) that can cause damage and harm to the surroundings, humans or other living species. It can occur when these substances are introduced at a faster rate than can be dispersed, diluted, decomposed or recycled. The major kinds of pollution are air pollution, water pollution, and land pollution. When considering environmental pollution, the most prevalent focus is on the use of fossil fuels and the subsequent carbon emissions contributing to the production of smog (the result of fossil fuel combustion combined with sunlight and heat), resulting ground-level ozone which can cause irritation to the respiratory system [4]. However, water pollution is also a major environmental concern and can occur in a number of ways. For example, chemicals in fertilisers used on agricultural land can gradually be washed into surrounding surface and groundwater systems by rainfall. Domestic households, industrial and agricultural practices produce wastewater (sewage), which is treated in a water treatment plant. The resulting treated waste is disposed of into the sea, and can cause pollution of many rivers and other watercourses.

\subsection{1: Wastewater Treatment Process}

According to the Urban Wastewater Directive (91/271/EEC), urban wastewater is defined as "domestic wastewater or the mixture of domestic wastewater with industrial wastewater and/or run-off rain water" [5]. For waste management this is treated by mechanical or biological 
means before being released as effluent from the waste water treatment plant (WWTP) with destinations that may include watercourses or agricultural land. This treatment is designed to reduce the risk of adverse impact to the environment through oxygen depletion of receiving waters, or ecosystem eutrophication by the increase in nitrogen and phosphates [6] is divided into three stages; primary, secondary and tertiary treatment. Primary treatment is concerned with the removal of suspended solids from the raw sewage using screening and sedimentation by gravity techniques, while secondary treatment is typically microbial digestion that encourages aerobic degradation of organic matter to carbon dioxide and water by pumping air throughout the wastewater. The treated water is separated from the residual sludge and is deemed suitable for discharge into the environment [7], while the sludge layer requires further clean-up. This may include liming or dewatering, producing a treated sludge deemed suitable for use as fertiliser for agricultural land, or used as feedstock for energy production [7,8]. A final, less common, tertiary treatment stage involves a disinfection step to destroy any residual micro-organisms through the application of ultraviolet light or chlorination with the resulting effluent discharged back into the water course or subjected to further treatment to produce drinking water [9].

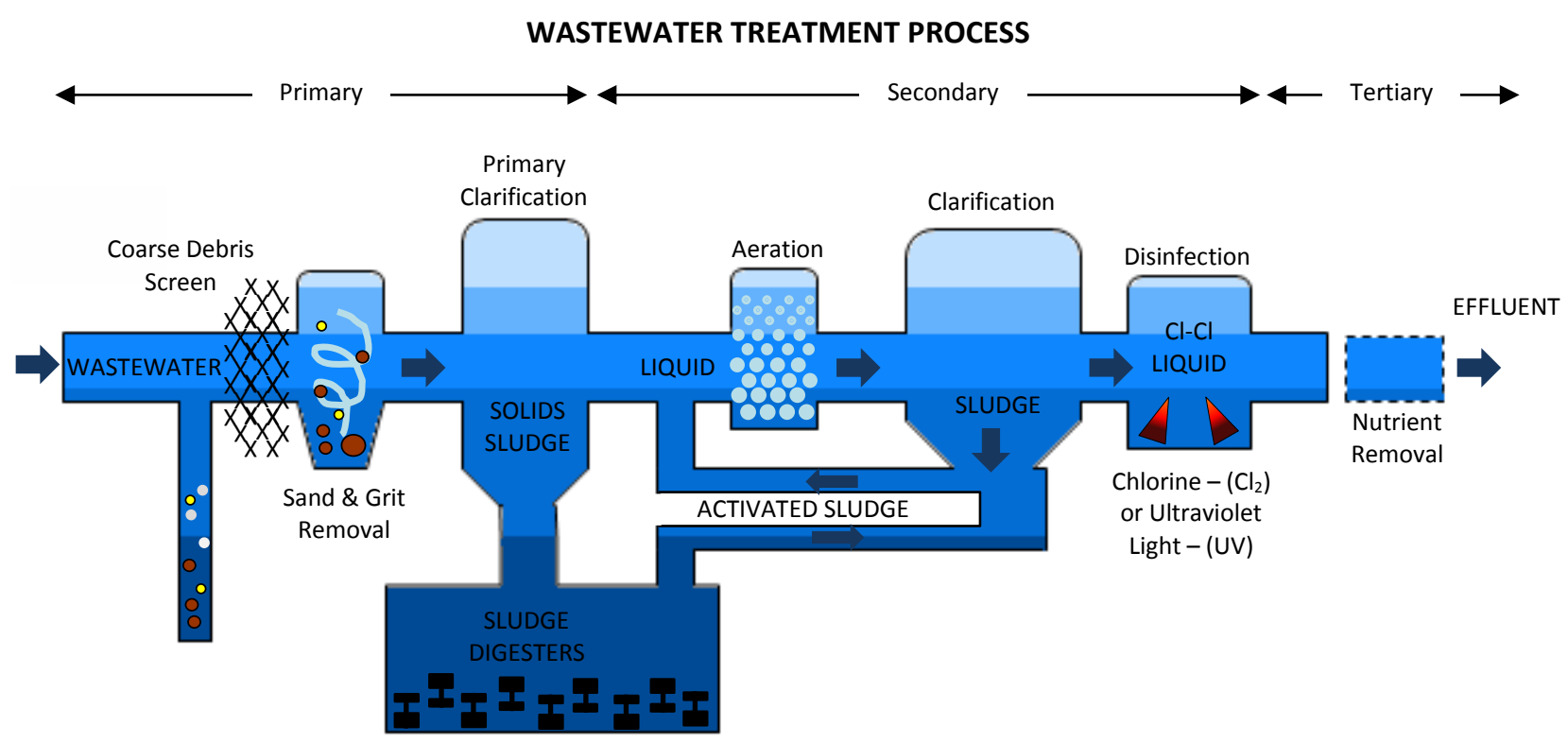

Figure 1.1: A schematic of the wastewater treatment process detailing the primary, secondary and tertiary stages of wastewater treatment, including removal of bio-solids, microbial degradation and disinfection, respectively.

Secondary treated sludge is nutrient-rich, making it a very appropriate fertiliser and this has been the favoured option of disposal since the Urban Wastewater Directive (91/271/EEC) proposed the phasing out of removal of sludge to surface waters in 1991 [5]. Since then, 80\% 
of sewage sludge is now recycled onto agricultural land in accordance with the procedures outlined in the Sludge (Use in Agriculture) Regulations 1989 and the Safe Sludge Matrix guidelines [6,10,11] published in 2001 .

\section{2: Current Regulatory Landscape for Environmental Analysis}

The publication of the Water Framework Directive, 2000/60/EC (WFD) in 2000 [12] was a new era for environmental monitoring. Member states were now obligated to look at the environment with a more holistic approach, considering the impact of environmental pollution on the ecosystem as a whole. This included water courses but also land and organisms living in these catchments such as biota. Information gathered from monitoring programmes following the introduction of this policy led to the development of environmental standards for hazardous substances, the Environmental Quality Standards Directive, 2008/105/EC (EQSD) in 2008. This directive detailed the maximum allowable concentration [13] in water samples before action must be taken for each compound deemed to be a hazardous substance (i.e. heavy metals and pesticides). However, this regulation was specific to water and did not consider wastewater effluent and its by-products, (i.e. sludge) or biota, or their potential impact on environmental pollution. Driven by this legislation the Chemical Investigation Programme (CIP) was established in 2009 [14], as a UK based initiative aiming to understand the prevalence of substances that may be potential pollutants in sewage samples, and to establish quality standards similar to those outlined in the EQSD (2008/105/EC) [13]. This initial CIP study was one of a number that directly fed into the amended environmental Directive, 2013/39/EU, which also encompassed findings from the WFD and EQSD. This legislation adopted a more complete approach to environmental monitoring considering alternative sample matrices and highlighted substances of emerging concern to the environment that are not yet subject to legislation in the form of a "watch list" (predominantly pharmaceuticals) [15]. This "watch list" functions differently to the priority substance list; here the obligation is to monitor the three nominated pharmaceuticals (with a maximum capacity of ten), considered to be hazardous, and gather data to determine the risk of these compounds within the environment. The candidates on this "watch list" may then be replaced or escalated to priority substance status depending on the outcome of the monitoring data. In 2015, a broader UK programme CIP II, was launched to investigate these pharmaceuticals and others identified as a potential environmental concern (see Table 1.1), focussing on their concentrations in environmental samples and again, to establish quality 
standards similar to those outlined within the EQSD, with proposed limits of detection for pharmaceuticals in sewage sludge of $0.1 \mathrm{mg} / \mathrm{kg}$, based upon the findings of the initial CIP study [16].

Table 1.1: The pharmaceuticals and metabolites currently classed as emerging substances within the Chemical Investigation Programme, II.

\begin{tabular}{|c|c|c|}
\hline Pharmaceuticals & Statins and Antifungals & Metabolites \\
\hline Diclofenac & Atorvastatin & 10,11- Epoxycarbamazepine \\
\hline Ibuprofen & Ortho-hydroxyatorvastatin & Norerythromycin \\
\hline Propranolol & Para-hydroxyatorvastatin & Norsertraline \\
\hline Atenolol & Azithromycin & \\
\hline Erythromycin & Clarithromycin & \\
\hline Metformin & Ciprofloxacin & \\
\hline Ranitidine & Benzotriazole & \\
\hline Carbamazepine & Tolyltriazole & \\
\cline { 1 - 2 } Sertraline & \multicolumn{2}{|}{} \\
\cline { 1 - 1 } Fluoxetine & \multicolumn{2}{|}{} \\
\cline { 1 - 1 } Tamoxifen & \multicolumn{1}{|}{}
\end{tabular}

\section{3: Persistent Organic Pollutants}

Persistent organic pollutants (POPs) are chemicals of global concern due to their potential for long-range transport, persistence in the environment (including air, water, soil and sediment), ability to bioaccumulate in ecosystems, as well as their significant negative effects on human health and other living species [17]. Humans are exposed to these chemicals in a variety of ways: mainly via the food chain, but also through the air we breathe. Many commonly used products may contain POPs, which have been added to improve product characteristics, such as flame retardants or surfactants. As a result, POPs can be found almost everywhere on our planet in measurable concentrations [17,18]. Many POPs were widely used during the post war industrial production boom when thousands of synthetic chemicals were introduced into commercial use, many of which proved beneficial in pest and disease control and crop production. Some of the more well-known POPs are pesticides, such as aldrin, dieldrin and dichlorodiphenyltrichloroethane (DDT) and industrial chemicals such as polychlorinated biphenyls (PCBs). However, due to the demands for environmental monitoring programmes, more candidate pollutants are being discovered in recycled waste with a significant potential impact for both environmental and public health, such as pharmaceuticals and other classes of biocides. There have already been global reports of the adverse effects of pharmaceuticals on the animal kingdom. For example, the non-steroidal anti-inflammatory, diclofenac, has 
caused multiple species of vulture in Asia to become critically endangered [19] with the Indian long-billed vulture and red-headed vulture populations decreased by 97-99\% [20]. The female contraceptive pill is another pharmaceutical with longstanding environmental impact; the feminisation of male fish due to expose to this particular hormone has caused a rapid decrease in population over a 2 year monitoring period [21]. Similarly biocides, such as triclosan and glutaraldehyde have been linked to a number of ailments, from skin irritation to breathing disorders, respectively [22,23], and the use of tributyltin (TBT), an antifouling agent, has been shown to have a long-lasting impact on marine eco-systems [24].

\subsection{1: Pharmaceuticals as Persistent Organic Pollutants in the Environment}

There are different classes of pharmaceuticals available for both human and veterinary use. The use of pharmaceuticals has risen year on year due to an ever aging population, the rise of chronic diseases and a general change in lifestyle. In Wales alone, the number of prescription items dispensed in 2016 totalled 80.3 million, a 1.0\% increase from 2015 [25]. Over-thecounter medicines, by comparison, are more difficult to regulate and determine exposure rates. While annual sales are recorded, there is no way to monitor how much is actually used and therefore estimating the potential quantity released into the environment, either as excretion products or by poor disposal of out-dated medication can make targeted analysis challenging. Those commonly administered include "over-the-counter" medicines such as non-steroidal anti-inflammatory drugs (e.g. ibuprofen), antihistamines (e.g. diphenhydramine), or prescription medications such as anticonvulsants (e.g. carbamazepine) and antibiotics (e.g. erythromycin). While drugs may group exert different effects on the body, they share several physiochemical characteristics, such as organic functionalities to encourage lipophilicity and polar groups, such as an alcohol, carboxyl or amino group to allow interaction with the target receptor. The drug lipophilicity is an important consideration for the environmental fate of a drug. This can be estimated by the octanol/water partition coefficient $\left(\log \mathrm{K}_{\mathrm{ow}}\right.$ or $\left.\log \mathrm{P}\right)$, as a measure of affinity to either organic or aqueous conditions, or more importantly for complex environments with sediment, by the solid-specific equilibrium sorption constant $\left(\mathrm{K}_{\mathrm{d}}\right)$, describing the distribution of a compound between sediment/sludge and water [26]. Values of $\mathrm{K}_{\mathrm{d}}$ can range from $10^{5}$ to less than 1 , and it is reported that high values of $\mathrm{K}_{\mathrm{d}}$ and $\log \mathrm{P}$ (usually a value greater than 3 ) are consistent with those compounds that can potentially adsorb to sludge, or bioaccumulate within soil and biota, respectively [27]. 


\subsection{2: Biocides as Persistent Organic Pollutants in the Environment}

There are two main classes of compound that are used to control and destroy bacterial growth, antibiotics and biocides. Antibiotics are typically derived from natural organic compounds and are effective in low concentrations with a specific single cellular target, whereas biocides are typically used against microbes on surfaces or in suspensions, applied at concentrations much greater than the minimum inhibitory concentration, unachievable with in vivo application [28]. The European biocide market amounted to approximately $€ 10-11$ billion, with an annual growth of $4-5 \%$ over the last 15 years, with a predicted increase over the coming years [29]. The uninhibited use of these compounds has led to an increasing concern of environmental exposure as the major disposal route of biocides is via drains and sewage system. It has been estimated that $50 \%$ of these biocides are degraded during the wastewater treatment process with $25 \%$ adsorbed to suspended solids and the remaining $25 \%$ dissolved into the water fraction [30,31]. As the treated wastewater effluents (i.e. solids and liquids) are recycled back into the environment, there is a potential for biocides to bioaccumulate and cause adverse effects on the ecosystem, with studies showing quaternary ammonium compounds in particular, are toxic to aquatic organisms at concentrations of approximately $1 \mathrm{mg} / \mathrm{L}$ [32].

Quaternary ammonium compounds (QACs) are broad spectrum, amphoteric surfactants used heavily in industrial and clinical applications, with increasing use in domestic cleaning products, such as laundry detergents, dishwashing liquids and disinfectants [33], over the last 10 years [34]. These cationic surfactants typically comprise of a positively charged nitrogen atom bonded to four carbon atoms, with at least one alkyl chain and form hygroscopic chloride or bromide salts. As a result of their structure, surfactants show solubility in polar and non-polar liquids and tend to adsorb at the phase interface, reducing the surface tension by disrupting interactions between solvent molecules. Finally, when the total surfactants concentration exceeds the critical micelle concentration, surfactants will aggregate into soluble structures, such as micelles, after which surface tension plateaus [33].

\subsubsection{1: Antimicrobial Resistance}

Quaternary ammonium compounds have been used extensively since the 1930s [34], and while there has been no evidence to show a reduction in effectiveness, studies have shown decreases in susceptibility of repeatedly exposed bacteria, as a result of hyper-expression of certain multi-drug efflux pumps [34,35], such as those associated with qacA-G genes in 
Staphylococcus aureus [36]. These efflux pump proteins are used to expel compounds that are damaging to the microbial cell and are found in both Gram-positive and Gram-negative bacteria. Activation of efflux pumps by biocides can also be effective on antibiotics, thereby increasing cross-resistance [37]. Another mechanism for simultaneous biocide and antibiotic resistance is co-resistance, and this occurs when multiple resistance genes are present [50]. Antibiotic resistance of bacteria can be increased by single-step mutations in the target enzymes or by neutralising enzymes, particularly beta-lactamases [38]. Given these mechanisms for both cross and co-resistance, there is a concern that an increase in multi-drug efflux pumps actively increase the frequency of mutational high-level resistance by allowing a greater proportion of organisms to survive antimicrobial exposure [38].

\subsection{Trace Analysis of Environmental Samples}

Since the implementation of WFD and CIP, there is a much broader range of environmental pollutants that require monitoring. The analysis of different chemistries at trace concentrations is one of the main challenges faced by the environmental industry. For trace quantitation, there first needs to be an effective and robust sample preparation method for the necessary sample clean-up, alongside sensitive and selective instrumentation. Liquid chromatography-mass spectrometry (LC-MS) is the gold-standard for environmental monitoring and trace quantitative analysis of non-volatile species, capable of reaching trace level sensitivity when used in combination with suitable sample preparation methods [39].

\subsection{1: Sample Preparation Techniques}

Sample preparation is an important stage of analytical analysis, especially when working with complex samples, such as biological or environmental matrices. By removing any interferences, such as salts or acids present in the matrix allows better sensitivity and recovery of the target analyte. The most commonly used sample preparation technique for environmental analysis is solid-phase extraction (SPE), however recent studies have shown that the Quick, Easy, Cheap, Effective, Rugged and Safe (QuEChERS) method has been investigated as an alternative to SPE [40,41,42].

\subsubsection{1: Solid-Phase Extraction}

Solid-phase extraction (SPE) is a sample clean-up technique generally used to selectively separate and concentrate a known target analyte within the sample. This preparation method 
has two modes of operation; it can work either by removing matrix interferences from a liquid sample using a solid sorbent (typically in cartridge form), or by retaining the target analyte which can be later eluted from the sorbent using appropriate solvents, also working as an effective concentration step (if the elution volume is less than loading volume). There are several sorbent chemistries available, with typical sorbents being silica based containing alternative functional groups bonded to the silanol surface [43]. Common SPE protocols used for environmental matrices have four main stages (see Figure 1.2):

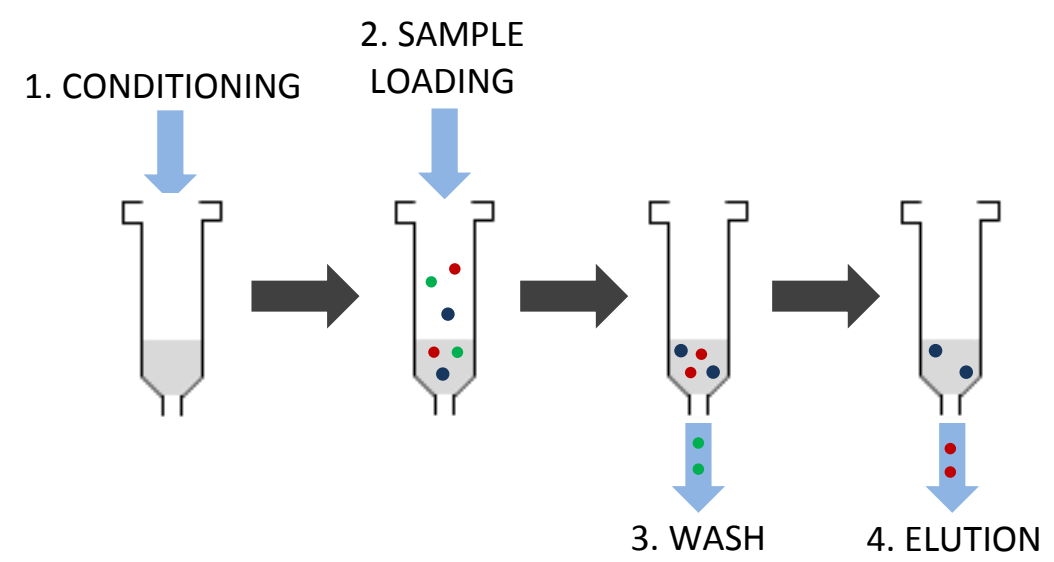

Figure 1.2: A diagram showing how a typical solid-phase extraction (SPE) is carried out. By retaining the sample components on the sorbent, matrix interferences can be selectively eluted before the analytes of interest are eluted for analysis.

1) Column pre-conditioning: Some sorbents require activation to solvate the column ready to interact with the target analyte. This is achieved by rinsing with a solvent of high elution strength, such as methanol or acetonitrile, followed by a rinse stage with the sample solvent, promoting the optimum environment for retention.

2) Sample Loading: The sample is loaded onto the cartridge where the target analytes are retained.

3) Wash: The cartridge is washed to remove interferences off the sorbent. This "wash" eluent can either be discarded, or saved for a secondary SPE procedure to ensure all of the analyte of interest is retained.

4) Elution: A suitable solvent of sufficient elution strength is used to displace the analyte of interest from the sorbent ready for analysis [44].

While SPE offers several advantages as a sample preparation method, including high selectivity and recovery of target analytes with good reproducibility, it can be complex when considering multiple analyte chemistries due to the need for extensive $\mathrm{pH}$ modification and 
despite there being a wide variety of sorbent types/ cartridge size available, the options can be costly. For example, one of the recognised methods for the analysis of complex environmental matrices such as soil and wastewater effluent (Environmental Protection Agency (EPA) Method 1694 [3]) requires the use of $20 \mathrm{~mL}$ Oasis HLB cartridges with $1 \mathrm{~g}$ of sorbent. These cartridges retail for approximately $£ 200$ for 20 , resulting in a cost per sample of approximately $£ 10$, which is undesirable for high throughput analysis.

\subsubsection{2: Quick, Easy, Cheap, Effective, Rugged and Safe (QuEChERS)}

The QuEChERS sample preparation method was developed in 2003 by Anastassiades and Lehotay for the extraction of pesticides from fruit and vegetables [45]. This is a two-step process involving a liquid partition into acetonitrile with the addition of drying agents (typically magnesium sulphate $\left(\mathrm{MgSO}_{4}\right)$ ) for dehydration and salts and buffers to limit polar interferences and facilitate phase transfer, followed by a dispersive solid-phase extraction (dSPE) for further interference removal, using sorbents such as primary-secondary amine (PSA), C18 and graphitised carbon black (GCB), as shown in Figure 1.3. At present, there are three standardised approaches, the original unbuffered method [34], the Association of Analytical Communities (AOAC) method [46] and the European Standard (EN 15662) method [47]. These methods were developed to incorporate a larger number of pesticides from a wider variety of fruit and vegetables, and each differ by the addition of buffers in the extraction kit; sodium acetate for the AOAC method, regulating the $\mathrm{pH}$ of the extraction solution to 3.6-5.6, and a combination of sodium citrate buffers for the EN 15662 method to regulate the $\mathrm{pH}$ of the sample to 3.0-6.2 [48]. Understanding the behaviour of the analyte in acidic and basic conditions using the acid dissociation constant ( $\mathrm{pKa}$ ) can ensure the compound is in a neutral state to achieve maximum recovery into the acetonitrile extraction solvent. Therefore, for samples that are $\mathrm{pH}$ sensitive, recoveries may be improved by using one of the adapted methods. Due to its low cost and resource requirement $[45,49]$, the QuEChERS method has undergone some initial investigation for sediment and soil samples to extract select pharmaceuticals, metabolites and pesticides [50,51,52], providing scope for further development for use with a larger suite of compounds from alternative matrices. 


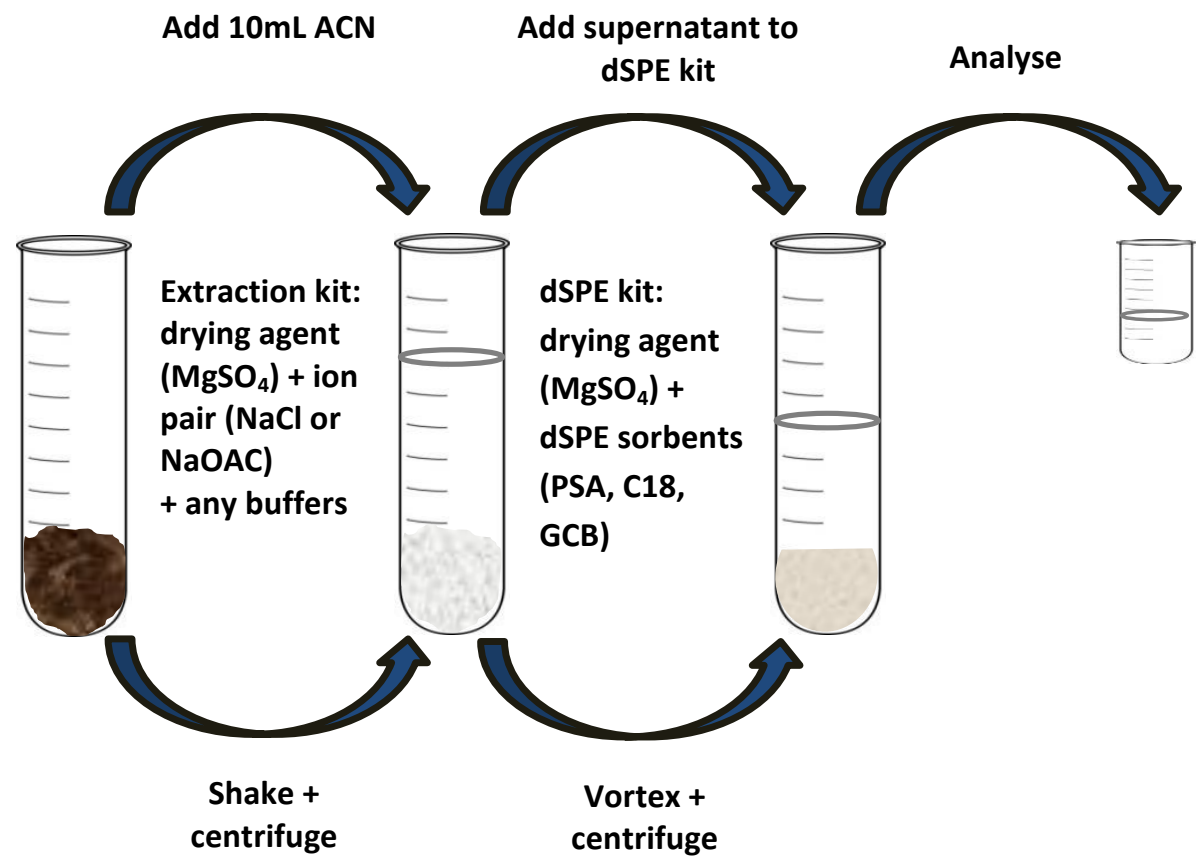

Figure 1.3: A diagram showing how the two-step QuEChERS sample preparation method is performed. Firstly, is the liquid partitioning into the acetonitrile (ACN) solvent, followed by a dispersive-SPE (dSPE) step, before the sample is ready for analysis.

\subsection{2: Liquid Chromatography (LC)}

While sample preparation can assist with the removal of matrix interferences, the final extract may still contain a complex mixture of different compounds. The most widely used separation technique used for quantitative analysis is reversed-phase liquid chromatography, which uses a non-polar (hydrophobic) stationary phase, like a C18 sorbent and a polar (aqueous) mobile phase such as water. Given this, it is considerably more versatile for a broad range of compounds with relatively inexpensive solvents that are safer than those used for normal phase.

\subsubsection{1: Principles}

Reversed-phase LC achieves separation of compounds within a mixture by the adsorption between a non-polar, hydrophobic stationary phase, packed into an analytical column, and a polar mobile phase [53]. The stationary phase consists of a packing material which differs in composition depending on the type of interaction required, for example, for environmental/pharmaceutical analysis a non-polar hydrophobic C18 column is typically used. Mobile phases are typically comprised of an aqueous mixture of water and a miscible polar solvent, such as acetonitrile or methanol, which solubilises the analyte retained on the stationary phase resulting in elution from the column. For compounds with poor retention on 
column, the addition of buffers or weak acids/bases can effectively improve compound retention by adjustment of the $\mathrm{pH}$ of the mobile phase. This adjustment can determine whether the compound of interest exist in the ionised or neutral form, where the ionised species elutes earlier from the column [53]. The mobile phase flow can be operated either isocratically, where there is a fixed mobile phase composition (i.e. 50\% mobile phase A:50\% mobile phase B) throughout the entire run, or using a gradient elution, where the proportion of the organic mobile phase solvent is increased throughout the run. Gradient elution is typically used when high levels of resolution are required for separation of a complex mixture to ensure elution of all compounds of interest, as analytes will be eluted sequentially in order of hydrophobicity.

\subsubsection{2: Chromatographic Parameters}

There are several factors that describe the performance of the chromatographic separation; column efficiency $(\mathrm{N})$, retention factor $(k)$, selectivity $(\alpha)$ and resolution $(\mathrm{R})$ [39].

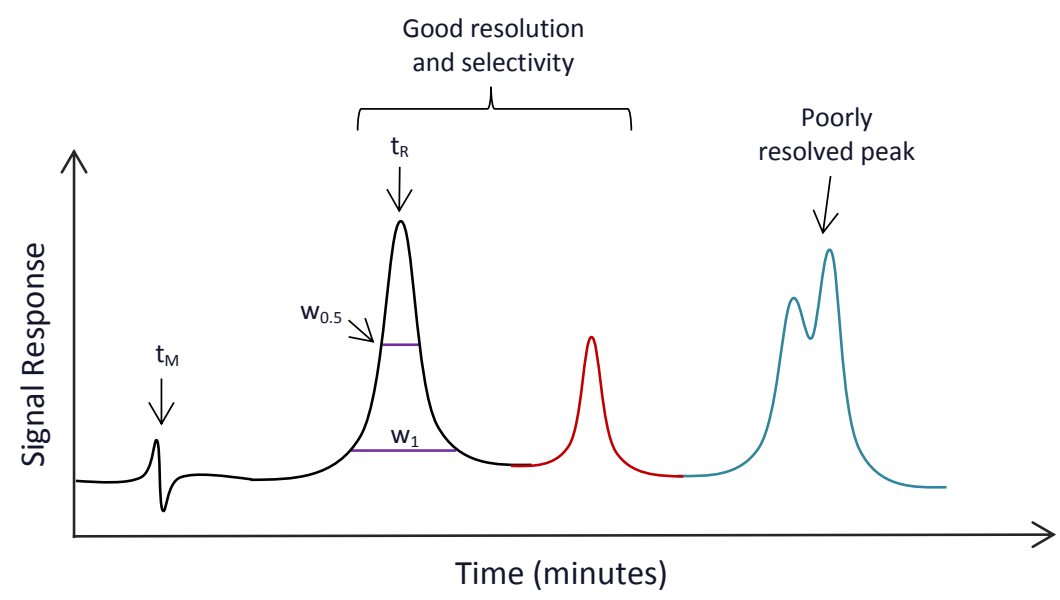

Figure 1.4: An example chromatogram displaying signal response versus time, demonstrating good separation of two retained compounds and an example of a poorly resolved peak, annotated with the following: retention time of the unretained compounds $\left(\mathrm{t}_{\mathrm{M}}\right)$, analytes $\left(\mathrm{t}_{\mathrm{R}}\right)$, and the peak width at the base $(\mathrm{W})$ or at half the maximum $\left(\mathrm{W}_{0.5}\right)$ are used to evaluate chromatographic performance.

1) Column Efficiency (N): This is also referred to as the number of theoretical plates, and can be related to the dispersion of the analyte band through the column. This can be dictated by the flow rate of the mobile phase and the column packing particle size (i.e. the diameter of the supporting silica beads). The smaller the particle size, the shorter the diffusion path length and time for the analyte, therefore decreasing the time spent inside the particle where peak diffusion can occur. 


$$
\mathrm{N}=16\left(\frac{t_{R}}{W}\right)^{2}=5.54\left(\frac{t_{R}}{W_{0.5}}\right)^{2}
$$

The relationship between column flow rate and efficiency is described by the Van Deemter relationship and this helps define the contribution of diffusion, defined by three main terms; eddy diffusion, longitudinal diffusion and mass transfer, shown in Figure 1.5.

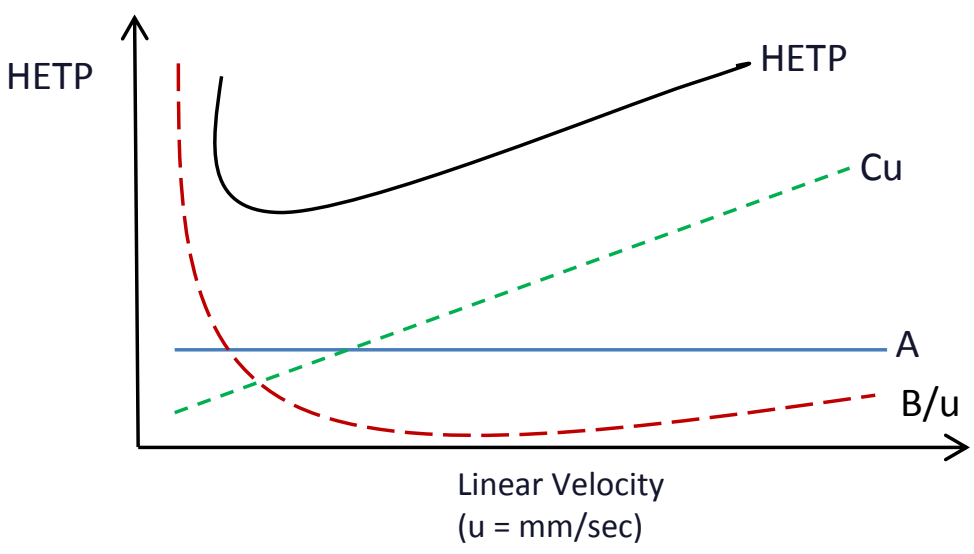

Where:

A - Eddy Diffusion

B - Longitudinal Diffusion

C - Mass Transfer

HETP - Height Equivalent

Theoretical Plate

Figure 1.5: A diagram of the Van Deemter relationship describing the effect of plate height (HETP) with linear velocity and the impact of analyte diffusion within the column.

Eddy diffusion (A) refers to the different paths an analyte in a "band" can take when travelling through the column. The paths available are due to the variation in the particle size of the column packing. These inconsistencies can lead to the analytes travelling through multiple pathways resulting in band broadening producing a broader peak shape therefore reducing the resolution of the separation. However, this can be reduced by using a column with smaller particle size [54].

Longitudinal diffusion (B) is related to the diffusion of the analyte contained in the injection solvent along the axis of flow and typically occurs when the internal volumes with the LC systems are larger than necessary [54]. For example, tubing that is too long, or has a wide internal diameter, or incorrectly connected zero dead volume fittings.

Mass transfer (C) refers to the speed of the mobile phase and the particle size and relates to the interaction of analyte molecules with the internal surface of the stationary phase and their distance of diffusion into and out of the pores of the packing material. As with eddy diffusion, the band broadening effect of mass transfer can be reduced by selecting a column with a smaller particle size. 
2) Retention Factor $(\boldsymbol{k})$ : The retention factor refers to the degree of retention of an analyte on column, and is defined by the time in which the analyte resides in the stationary phases relative to the time it resides in the mobile phase. Compounds with low retention often have varying retention time, making analysis irreproducible, while compounds that have high retention can exhibit peak broadening due to strong retention with the stationary phase. This can be controlled by the polarity of the mobile phase.

$$
k=\frac{\mathrm{t}_{\mathrm{R}}-\mathrm{t}_{\mathrm{M}}}{\mathrm{t}_{\mathrm{M}}}
$$

3) Selectivity $(\boldsymbol{\alpha})$ : Selectivity is the measure of separation of two analytes, and can be controlled by the type of column and mobile phase composition used.

$$
\alpha=\frac{k_{1}}{k_{2}}, k_{2}>k_{1}
$$

4) Resolution $\left(\mathbf{R}_{\mathbf{s}}\right)$ : Resolution refers to the degree to which two compounds are separated. This is determined by the selectivity and column efficiency. Poor resolution can lead to a co-elution of analytes, shown in Figure 1.4.

$$
\begin{gathered}
\mathrm{R}_{\mathrm{s}}=\frac{\sqrt{\mathrm{N}}}{4}\left(\frac{\mathrm{k}}{\mathrm{k}+1}\right)\left(\frac{\alpha-1}{\alpha}\right) \\
\mathrm{R}_{\mathrm{s}}=\frac{2\left(\mathrm{t}_{\mathrm{R}, 2}-\mathrm{t}_{\mathrm{R}, 1}\right)}{\mathrm{W}_{1}+\mathrm{W}_{2}}=\frac{1.18\left(\mathrm{t}_{\mathrm{R}, 2}-\mathrm{t}_{\mathrm{R}, 1}\right)}{\mathrm{W}_{0.5,1}+\mathrm{W}_{0.5,2}}
\end{gathered}
$$

\subsubsection{3: Column Characteristics for Reversed-Phase Chromatography}

Typically columns consist of a non-polar, hydrophobic stationary phase and while C18 bonded silica is the most commonly used column type, a number of modifications are available to improve retention and selectivity of more challenging analytes. This can be achieved by alteration of the silica surface by bridging the silica and C18 chain with ethylene (bridged ethylene hybrid (BEH)), which provides a wider operational $\mathrm{pH}$ range ( $\mathrm{pH} 1-12)$ due to increased chemical stability of hybrid particle, or by the application of a small charge to the surface of the bead, (charged surface hybrid (CSH)) leading to improved performance of basic compounds with acidic, low ionic strength mobile phases (i.e. acetonitrile). 
Endcapping is commonly used to improve chromatography, and refers to the derivatisation of accessible silanol groups in a bonded stationary phase with trimethylsilane (TMS) to prevent peak tailing, common to polar compounds. New technology has been developed to use a trifunctional C18 alkyl phase bonding (T3) compatible with high-aqueous mobile phase conditions and to promote polar compound retention due to low-ligand density, enabling analytes to more readily access the pore structure.

\subsection{3: Mass Spectrometry (MS)}

Mass spectrometers are the most sensitive and selective detector used in conjunction with LC analysis. While LC separates compounds within a mixture by their physico-chemical properties, MS differentiates compounds by mass, specifically their mass-to-charge ratio $(\mathrm{m} / \mathrm{z})$ and provides the capability to identify the species corresponding to each chromatographic peak through its unique mass spectrum.

\subsubsection{1: Principles}

Mass spectrometry is used to analyse the mass of a gaseous ion under vacuum and separate those ions based upon the molecular $\mathrm{m} / \mathrm{z}$, which is plotted against relative abundance as a mass spectrum. A mass spectrometer comprises of four main components, and inlet, and ionisation source, a mass analyser and a detector [55]. The inlet is where the sample is introduced into the mass spectrometer; for example, when coupled with an LC system, the eluent is directly connected from the end of the analytical column into the ionisation source. The ionisation source generates gaseous ions from sample molecules delivered by the inlet. When coupled to an LC system, the most common interfaces are atmospheric pressure chemical ionisation (APCI) or electrospray ionisation (ESI). These sources negate the difficulties in interfacing a liquid phase (i.e. LC) into a gas phase technique (i.e. MS) as the atmospheric pressure source can tolerate flow rates up to $1 \mathrm{~mL} / \mathrm{min}$, typical of LC analysis [55]. The mass analyser is the vacuum chamber in which separation of ions according to their $m / z$ occurs. Similarly with ionisation sources, there are multiple mass analysers to choose from depending on the needs of analysis being performed; quadrupole mass spectrometers are good for quantitation due to their fast scanning capabilities and robust operation, while ion traps are useful for rich qualitative data sets containing multiple stages of fragmentation of an analyte. Finally there is a detector, typically an electron multiplier, which detects the ions and amplifies them into a signal that can be used to produce a mass spectrum. The resulting mass 
spectrum can then be used to determine the presence of isotopes, chemical structure and with high resolution instruments, the accurate mass of the ion.

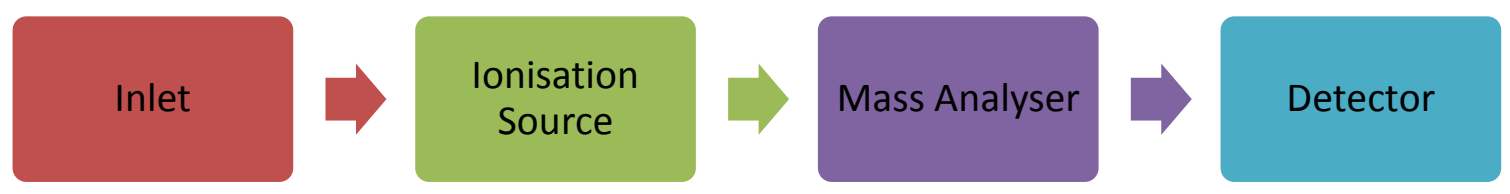

Figure 1.6: Schematic of a basic mass spectrometer showing three main sections; an ionisation source, mass analyser and detector.

\subsubsection{2: Ionisation Sources}

\subsubsection{1: Electrospray Ionisation}

Electrospray is a soft ionisation technique which results in little fragmentation and can be used for both positive and negative ions. It is typically used for the analysis of polar molecules, which, through the use of $\mathrm{pH}$ modifiers, forms characteristic ions such as $[\mathrm{M}+\mathrm{H}]^{+}$, $[\mathrm{M}+\mathrm{Na}]^{+},[\mathrm{M}+\mathrm{Cl}]^{-}$and $[\mathrm{M}-\mathrm{H}]^{-}$. This is achieved by the application of an electric field to a liquid sample passing through a capillary tube, creating a fine spray of highly charged droplets. As the droplets decrease in size due to solvent evaporation through the use of heat and a desolvation gas $\left(\mathrm{N}_{2}\right)$, the charge density on the droplet surface increases causing a deformation of the droplet into a Taylor Cone, eventually releasing many smaller droplets by repetitive Coulombic explosion [56]. This process occurs repeatedly until droplet sizes of 20 $\mathrm{nm}$ [57] are reached, at this stage two theories are proposed; and the ion evaporation model (IEM) and the charge residue model (CRM) [56, 58]. The IEM, proposed by Iribarne and Thomson, suggests that once a droplet with a radius of between 10-20 nm reaches its Rayleigh limit (the maximum amount of charge a liquid droplet can carry before ejecting fine jets of liquid), the electric field on the surface of the charged droplet is high enough that the solvated ions are released from the droplet directly into the gas phase. The CRM, proposed by Dole and Röllgen, states that as the solvent evaporates from the droplet, the decrease in size causes the charge density to exceed the surface tension of the droplet causing Coulombic fission, producing several smaller droplets. Successive fissions result in the formation of nanodroplets that contain a single analyte ion. Electrospray ionisation can be particularly prone to matrix suppression with ionisation occurring through adduct formation. Thus, for adduct formation through proton ionisation will favour molecules with the greatest proton affinity, thereby suppressing molecules with a lower affinity. It is therefore, key that the degree of matrix effects is considered and addressed when using this ionisation technique 
with complex samples and for applications requiring a reliable signal that is representative of a measured amount (i.e. quantitation).

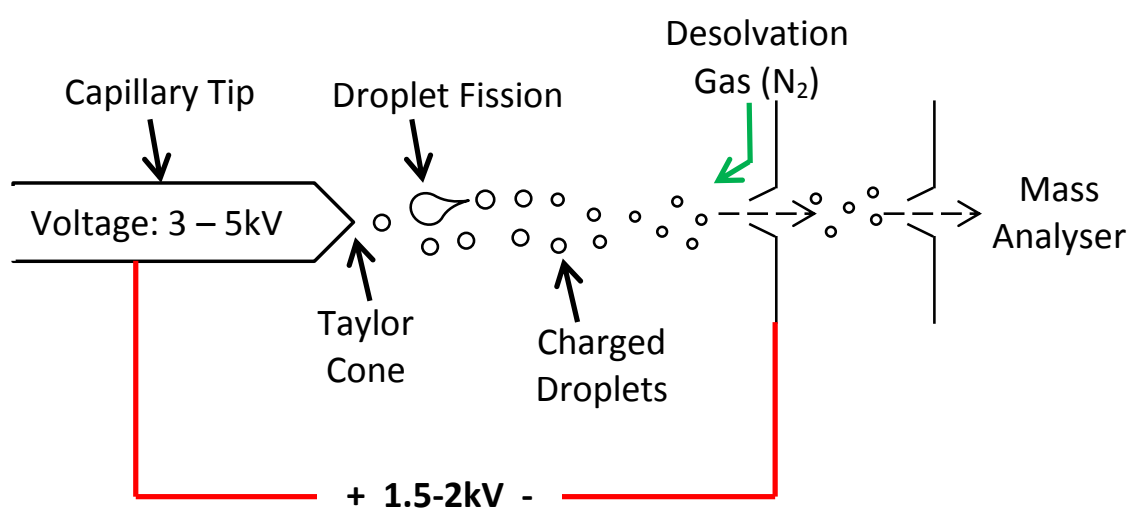

Figure 1.7: Schematic of an electrospray ionisation source showing the nebulised spray from the capillary tip and the fission of liquid droplets forming gaseous ions into the mass analyser.

\subsubsection{3: Mass Analysers}

\subsubsection{1: Quadrupole Mass Analyser}

A quadrupole is a scanning mass analyser consisting of four parallel circular or hyperbolic rods, to which a high-frequency oscillating electric field is applied. Ions are introduced into the analyser from the ionisation source and travel in the z-direction (see Figure 1.8). Ions are then separated according to their $\mathrm{m} / \mathrm{z}$ as a result of alternating a direct-current (DC) and radiofrequency (RF) voltages. When the DC potential is applied to the positive pair of electrodes, ions accelerate towards the centre of the quadrupole. The oscillating voltage of positive and negative charge causes ions to successively be attracted and then repelled from each rod, therefore drawing the ions through the quadrupole. The simultaneous action of these voltages enables a stable trajectory for ions of a certain $\mathrm{m} / \mathrm{z}$, causing them to reach the detector for a given ratio of voltages: other ions have unstable trajectories and will collide with the rods. This allows the operator to scan for a range of $m / z$ values by continuously varying the applied voltage [59]. An alternative, more selective mode includes single ion monitoring (SIM). This requires the quadrupole to be fixed on a specific RF/DC voltage, enabling the stable trajectory of the relevant $\mathrm{m} / \mathrm{z}$. This more specific approach increases the frequency of measurement at the relevant $\mathrm{m} / \mathrm{z}$, subsequently increasing the signal-to-noise ratio and the sensitivity. Therefore, LC-MS using a quadrupole provides a sensitive and selective means of ion detection. 


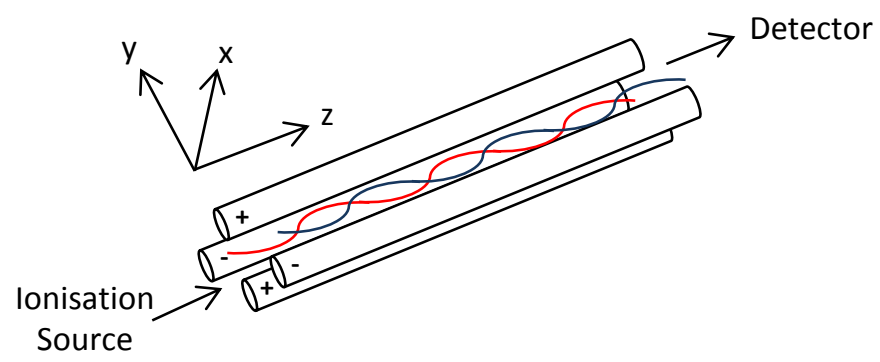

Figure 1.8: Schematic of a single quadrupole mass analyser showing the path of an ion with a stable trajectory through the quadrupole.

\subsubsection{2: Ion Trap Mass Analyser}

The quadrupole ion trap mass analyser consists of three electrodes to confine ions; two endcap electrodes and a central ring electrode. When subjected to an electric field created by a RF voltage, the ions are held inside the trap and take on an oscillating path related to their $m / z$, forming a figure-of-eight shaped trajectory, known as a Lissajous figure [60,61]. A dampening gas, typically helium, is used to stabilise the ion trajectories towards the centre of the trap, preventing any loss of ions through collision or coalescence by removing excess energy through collision. Ion stability is based upon the Mathieu equations which, when simplified shows that the stability of any ion of a given $\mathrm{m} / \mathrm{z}$ depends upon the parameters ' $\mathrm{a}$ ' and ' $\mathrm{q}$ ', relating to DC and RF voltages, respectively, as shown in the following equations:

$$
\begin{aligned}
& \mathrm{q}=\mathrm{k} \frac{\mathrm{V}}{\mathrm{m} / z} \\
& a=0
\end{aligned}
$$

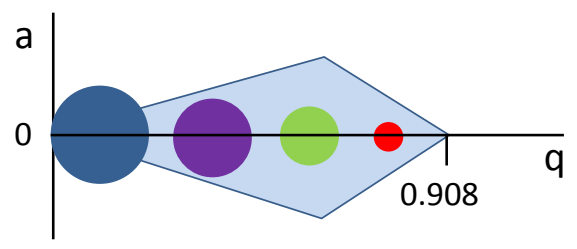

Figure 1.9: A visual representation of the Mathieu equations (also shown) relating to ion stability within an ion trap. 0.908 is the critical point at which the ions would become unstable in both the $\mathrm{x}$ and $\mathrm{y}$ directions.

As ' $a$ ' is constantly set to zero, the 'q' value is proportional to an ion's $m / z$, with smaller ions will having a larger ' $q$ ' value, and therefore be ejected from the trap first. An AC voltage, known as the resonance ejection voltage, is applied to prevent the ions from becoming unstable in both the $\mathrm{x}$ and $\mathrm{y}$ directions, then the instrument ramps the RF amplitude from a low voltage to a high voltage, causing the 'q' values of the ions to increase and the ions start moving towards the edge of the stability diagram and scan out of the trap [62]. 


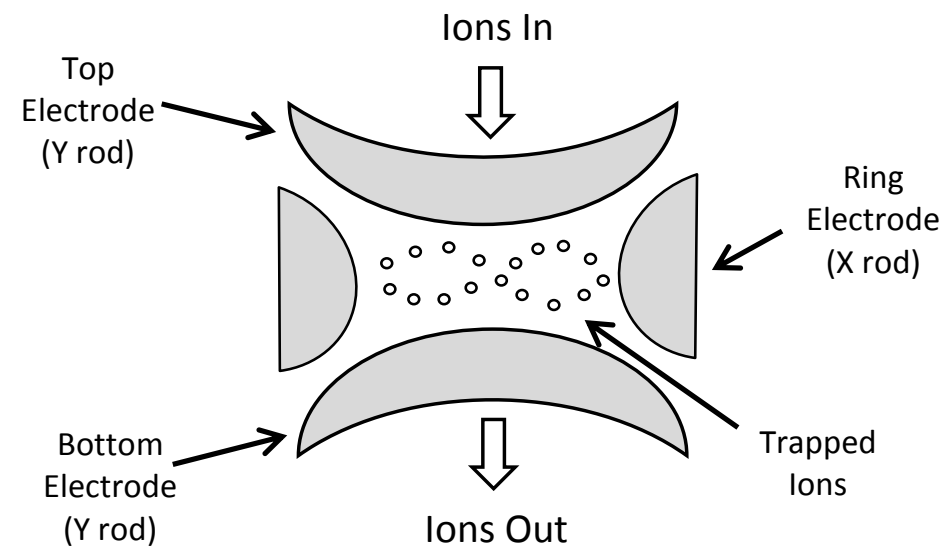

Figure 1.10: Cross-sectional schematic of a quadrupole cubic ion trap mass analyser showing a central ring electrode with a top and bottom end cap electrode, containing ions oscillating in a figure-of-eight orbit.

\subsubsection{3: Orbitrap Mass Analyser}

The Orbitrap is an electrostatic ion trap and consists of two endcap electrodes, and an inner "spindle" shaped electrode. A DC voltage is applied to the spindle electrode, resulting in a high static voltage between the two endcap electrodes. When ion packets enter the Orbitrap, they are trapped by their attraction to the spindle electrode, which, contrasted by their inertia begin to orbit around the inner electrode, oscillating between the two outer electrodes. The ions separate into discrete bands that are determined by their differing masses, and $\mathrm{m} / \mathrm{z}$ measurements are delivered as a function of oscillation frequency using Fourier Transforms (FTs) [63]. The translation of these frequencies into $\mathrm{m} / \mathrm{z}$ values and their amplitudes into intensities relates to the resolution of the mass spectrum obtained, whereby the longer the transient signal is recorded, the higher the resolution. This allows for high resolution measurements of up to 500,000 full width-half maximum, and accurate mass measurements with $<1 \mathrm{ppm}$ mass accuracy.

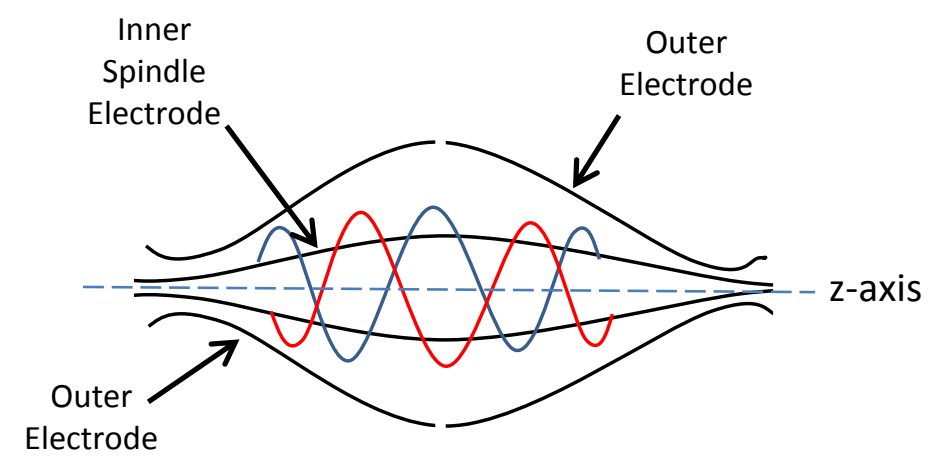

Figure 1.11: Cross-sectional schematic of an Orbitrap mass analyser showing the two outer electrodes and the central "spindle" electrode, containing ions orbiting the central electrode along the z-axis. 


\subsubsection{4: Quadrupole Time-of-Flight Mass Analyser}

To maximise the sensitivity and resolving power of mass spectrometry, hybrid instruments such a quadrupole time-of-flight (Q-ToF) mass spectrometer are commonly used for the acquisition of high resolution and high accuracy data. A Q-ToF comprises of a combination of a quadrupole (and collision cell) and a time-of-flight mass analyser, where ions are separated according to $\mathrm{m} / \mathrm{z}$ derived from the time taken to reach a detector at a known distance in either a linear or reflectron configuration, with the latter being more typical of high resolution analysis due to the increased flight path of the ion.

Typical operation of a Q-ToF involves the introduction of ions into a mass filter, followed by a collision cell in which fragmentation can occur by applying an RF voltage to the rods. This creates an electromagnetic field confining ions above a particular mass to the centre of the rods and collision induced dissociation (CID) occurs using a collision gas, such as argon [64]. Ions then exit the collision cell as an ion beam, and pass through into the ToF analyser and into the ion pulser, which consists of multiple stacked plates to which a high voltage is applied, accelerating the ions through a low pressure flight tube. An electrostatic ion mirror is used to reflect the ions back towards the multi-channel plate detector (MCP) at the top of the flight tube. As all similarly-charged ions have the same kinetic energy, those with low mass show greater velocity and therefore reach the detector first. Since mass (m), charge, and kinetic energy (KE) determine the arrival time of an ion at the detector, the ion's velocity (v) can be represented using the following equation:

$$
\mathrm{v}=\frac{\mathrm{d}}{\mathrm{t}}=\left(\frac{2 \mathrm{KE}}{\mathrm{m}}\right)^{\frac{1}{2}}
$$

Where, $\mathrm{d}$ is the given distance travelled by the ions, $\mathrm{t}$ is the time taken to for the ion to reach the detector, where $t$ depends on the $m / z$ [65]. 


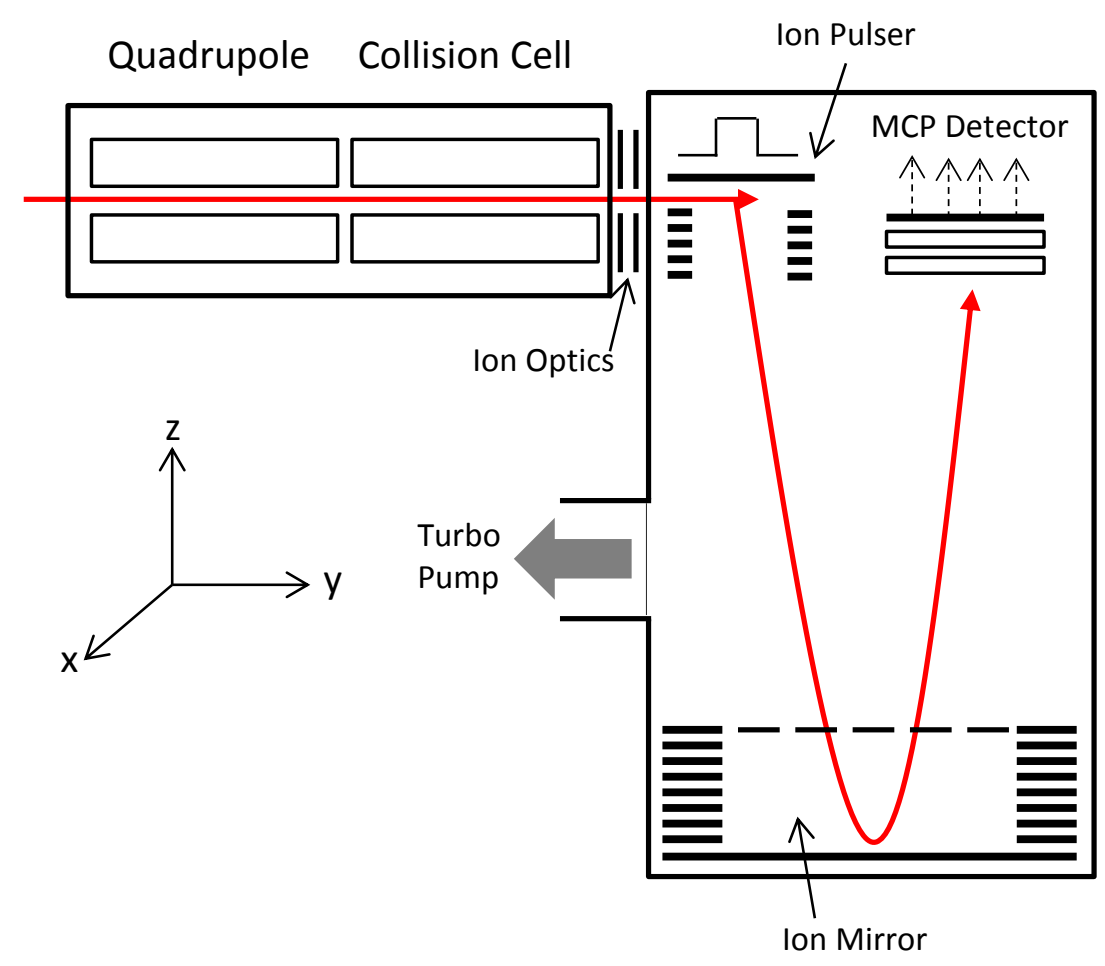

Figure 1.12: Schematic of a typical Q-ToF mass analyser, showing the path of an ion through the quadrupole and collision cell and the flight path when operated in reflectron mode.

\subsubsection{4: Data Acquisition}

To carry out qualitative and quantitative analysis using a mass spectrometer, several scan modes can be applied depending on the needs of the experiment. For qualitative analysis, there are multiple scan types that can be used, for example, a full mass scan, a product ion scan or data dependant analysis. A full mass scan is used to record all ions over a selected mass range, and is useful to aid identification of unknown compounds as it can give information regarding the sample composition [59]. A product ion scan is more selective, focussing on recording all product ions from a single precursor ion $\mathrm{m} / \mathrm{z}$. Similarly a data dependant acquisition (DDA) can be performed, whereby a number of precursor $m / z$ recorded in a survey scan can be selected using predetermined rules and subjected to a second stage of mass selection in an MS/MS analysis, typically achieved by collision induced dissociation (CID) with a neutral species (i.e. helium or argon gas). Quantitative analysis typically requires a more selective approach to data acquisition, whereby a single ion monitoring (SIM) scan, or a selected/multiple reaction monitoring (SRM/MRM) scan is used. A SIM scan is used to measure a single ion's $m / z$ rather than the whole mass range and can result in an enhancement of sensitivity versus a full mass scan, lending itself well to quantitative analysis. An SRM or MRM is used to record a specified reaction pathway of an ion of 
interest, increasing specificity and further enhancement in sensitivity of the measurement versus a SIM or full mass scan.

\section{5: Current Research}

\subsection{1: Pharmaceuticals in Wastewater and Sludge}

The majority of research that has been carried out regarding the detection of pharmaceuticals in the environment involves the analysis of water samples, with very little in the UK focussing on wastewater. The research regarding wastewater typically involves the use of SPE as the sample preparation method with the standard protocol published by the Environment Protection Agency (EPA), Method 1694 [3] also recommending this approach. However, there have been difficulties with the widespread adaption of this method with UK regulatory agencies (e.g. Environmental Agency and Natural Resources Wales) using alternative methods that are laborious, some taking days of preparation. Work carried out inhouse has also shown further challenges with ineffective results for sludge samples collected within the UK. Of the studies carried out in Europe, a range of methods have been used for various pharmaceuticals but not specific for CIP II. In a study carried out by Gracia-Lor et al. [66], 19 samples of effluent wastewater were tested from different WWTP around Spain, and 37 out of 47 pharmaceuticals investigated were detected at least once. The highest concentrations reported were $200 \mu \mathrm{g} / \mathrm{L}$ for acetaminophen and $15 \mu \mathrm{g} / \mathrm{L}$ for ibuprofen, which are both well above the recommended detection limits outlined in CIP II. Similarly for a study carried out by López-Serna et al. [67], the concentrations detected in river water collected downstream from WWTP for non-steroidal anti-inflammatory drugs such as naproxen, ibuprofen and acetaminophen also exceeded these recommended maximum concentrations with 109, 541 and $872 \mathrm{ng} / \mathrm{L}$ reported, respectively.

Another extensive study looked at 81 pharmaceutical residues and some of their metabolites in Spanish surface waters, and both secondary and tertiary wastewater [68]. Again, SPE was used as a sample preparation technique and analysed using UPLC-MS with an ESIquadrupole linear ion trap mass spectrometer. The method was validated to determine the method detection and quantitation limits (MDL and MQL), as well as matrix effects and the recovery, with the latter showing values greater than $50 \%$ for most compounds. However, only selected compounds were investigated for matrix effects by comparing the drug response in matrix versus a solvent based sample. For these compounds it was reported that high levels of ionisation suppression occurred (20-90\%). Despite these deficiencies when the 
method was applied to effluent wastewater after secondary biological treatment 40 of the 81 pharmaceuticals targeted were still detected at concentrations ranging from $\mathrm{ng} / \mathrm{L}$ to low $\mu \mathrm{g} / \mathrm{L}$, well above the MQL of 1-50 ng/L, highlighting that concentrations of pharmaceuticals within wastewater samples could be severely underestimated and further method improvement is needed to assess actual pharmaceutical concentrations.

More recent studies have been carried out to determine the bioaccumulation of pharmaceuticals within wastewater treatment effluents $[40,69,70]$. A study carried out by Kachhawaha et al. [40] investigates the use of a QuEChERS-based extraction process on sewage water from a WWTP in India. A total of six pharmaceuticals were detected, metformin, acetaminophen, atenolol, carbamazepine, methylparaben and triclosan, with concentrations between 0.1-13.4 ng/mL, with the most abundant being acetaminophen and metformin at 6.9 and $13.4 \mathrm{ng} / \mathrm{mL}$, respectively. These concentrations, while less than those observed in studies of sewage sludge, show that pharmaceuticals have the ability to accumulate within aqueous wastewater fractions.

A study carried out by Luque-Muñoz et al. [69] investigated the concentrations of a selection of pharmaceuticals within compost derived from sewage sludge in Spain; a method of recycling that is said to reduce the concentrations of PPCPs over time [69]. Using an saltassisted liquid-liquid extraction method, similar to the salting out step of a QuEChERS extraction, concentrations of ketoprofen, methylparaben, diclofenac and flufenamic acid were reported to be the most abundant at 510, 240, 175 and $128 \mathrm{ng} / \mathrm{g}$, respectively. A study carried out in the Slovak Republic reports similar concentrations of commonly prescribed and illicit drugs from five wastewater treatment plants [70], with the highest concentrations found to be 1300, 800 and $580 \mathrm{ng} / \mathrm{g}$ for fexofenadine, verapamil and citalopram, respectively. Other compounds detected include diclofenac, carbamazepine, acetaminophen, codeine, cannabinol and MDMA (concentrations between 3.3-330 ng/g). These studies further support the need to determine the pharmaceutical content of wastewater samples within the UK, characterising any matrix effects and the absolute recovery of analytes, to ensure accurate quantitation in complex matrices.

\subsection{2: Biocides in Wastewater and Sludge}

Similarly to the pharmaceuticals, there is little research regarding the detection of QACs in wastewater, with no studies based in the UK. Of the studies carried out; one in Austria and one in China, a range of methods have been used, typically sample preparation methods 
involving the use of liquid-based extractions, such as Soxhlet extraction and SPE In a study carried out by Martínez-Carballo et al. [71], 21 samples of river sediment and 6 samples of sludge were tested from different WWTP around Austria using a Soxhlet extraction method, involving $150 \mathrm{~mL}$ of acidified methanol over an 18 hour time period. Of the 12 QACs analysed (alkyl benzyl, dialkyl and trialkyl QACs), benzalkonium salts (BACs) and dialkyldimethylammonium salts (DDACs) were detected in the highest concentrations, with maximum concentrations of 3.6 and $2.1 \mathrm{mg} / \mathrm{kg}$ for BAC-C12 and DDAC-C18 in sediment, respectively. Within the sludge samples, DDAC-C18 was quantified with a mean concentration of $10 \mathrm{mg} / \mathrm{kg}$, however the $\mathrm{BAC}$ compounds and the trialkylammonium salts (ATACs) were also detected, but at lower concentrations of between $0.16-8.4 \mathrm{mg} / \mathrm{kg}$. This confirms the need to study QACs in wastewater samples, and also highlights the need for an alternative sample preparation method, more suited to high-throughput monitoring analysis. Another study carried out in China, looked at 17 QACs within 52 samples of digested sludge collected from WWTP around the country [72]. Samples were extracted using a two-step liquid extraction, first with $10 \mathrm{~mL}$ of methanolic hydrochloric acid then with $10 \mathrm{~mL}$ of chloroform before being passed through an anion exchange resin. Total concentrations were similar to those seen in the Austrian study, with concentrations of ATACs, BACs and DDACs found to be in the range of 0.38-293, 0.09-344 and 0.64-344 mg/kg, respectively.

A more recent study investigated the concentrations of biocides, including BACs, within Swedish sewage sludge and wastewater [73]. Samples of digested sludge and treated effluent were extracted using SPE before LC-MS/MS analysis. Concentrations of biocides within the digested sludge samples were found to be the highest with BAC-C10 to C16 observed in the range of $0.1-35 \mathrm{mg} / \mathrm{kg}$, with the most abundant biocide found to be hexadecyltrimethylammonium bromide (HDTMA) at $79 \mathrm{mg} / \mathrm{kg}$. Surprisingly, each of the biocides studied were detected within the treated effluent samples, despite their preference to adsorb to biosolids. Concentrations were predictably much lower, with BAC-C12 and HDTMA found to be the most abundant at 66 and $72 \mathrm{ng} / \mathrm{L}$, respectively, and concentrations of between 2 and $30 \mathrm{ng} / \mathrm{L}$ were recorded for BAC-C10, C14 and C16.

The high concentrations determined within these studies further highlights the need to determine QAC concentration within UK wastewater to inform WFD. 


\subsection{3: Pollutants in Biota}

A concern arising from studies investigating the detection of pharmaceuticals in water is the potential of drugs to bioaccumulate in biota, specifically filter feeders such as shellfish, and the possibility of drug transfer into the food chain. Research in this area is sparse with only a few publications investigating these concerns within the UK, with no published research concerning the detection of QACs within biota. Of the most notable studies, McEneff et al. [74] in 2013, investigated the bioaccumulation of pharmaceuticals in cooked and uncooked bivalves (blue mussels) collected from a pristine site in west Ireland. The five compounds chosen for the study, diclofenac, mefenamic acid, trimethoprim, carbamazepine and gemfibrozil, were administered to the bivalves by direct injection of a $10 \mathrm{ng}$ pharmaceutical mixture or by daily exposure via spiked artificial seawater. Samples of freeze-dried cooked and uncooked bivalve tissue were homogenised and prepared using a pressurised liquid extraction followed by solid-phase extraction. The results showed that, with the exception of trimethoprim (a basic antibiotic), the concentrations of the pharmaceuticals increased after the bivalves were steam cooked, with the biggest increase from $1.6 \mu \mathrm{g} / \mathrm{g}$ to $89.6 \mu \mathrm{g} / \mathrm{g}$ observed for mefenamic acid. This result indicates the capture of drug within the tissue and correlates with studies of pesticides and heavy metals in other foods. For example, concentrations of the pesticide hexachlorobenzene in meat and fish increased significantly when cooked [75] along with the concentrations of heavy metals when in seafood [76]. This is reportedly due to the loss of water encountered during cooking acting as a concentration step. This effect is an important factor that must be considered when investigating both the amount of "free" pharmaceutical within biota samples and any (eco)-toxicity studies that may inform subsequent environmental and human risk assessments during drug development and environmental impact work. This study highlights the potential of pharmaceuticals to accumulate within biota under controlled conditions however, there is little evidence to determine the level of pharmaceutical contamination within biota exposed to wastewater effluent.

\section{6: Research Need}

The environmental persistence of organic pollutants, such as pharmaceuticals and biocides, is a growing area of research. Until the introduction of the Water Framework Directive (2000/60/EC) and Environmental Quality Standards (Directive 2008/105/EC), the impact of drug emission into the environment through wastewater treatment plants has been largely 
unconfirmed and unrestricted. Preliminary research has shown that compounds with high octanol-water partition coefficient $\left(\mathrm{K}_{\mathrm{ow}} / \log \mathrm{P}\right)$, like many common pharmaceuticals and biocides, are not biodegraded during wastewater treatment and are able to bioaccumulate, adsorbing to soils and sludge. As treated sludge is routinely deposited on land, it is important to understand the extent of any chemical accumulation. Current recognised methods for preparing complex environmental matrices such as soil and wastewater effluent for analysis are typically multi-step procedures using a range of techniques and apparatus, resulting in methods that are time and resource consuming, unsuitable for high-throughput analysis. Focussing on compounds of interest to the Chemical Investigation Programme; a British research initiative concentrating on the monitoring of pollutants in sludge, and those detected in preliminary in-house data (see Appendix 1.1), we propose to develop a suitable sample preparation method for the simultaneous extraction of a selection of pharmaceuticals and biocides, commonly used in a domestic capacity (see Table 1.2) from complex environmental matrices including water, soil, sludge and biota, based upon the QuEChERS (Quick, Easy, Cheap, Effective, Rugged and Safe) methodology. Compounds were chosen according to their lipophilicity (i.e. $\log \mathrm{P}$ ), based on the predication that compounds with a $\log \mathrm{P}$ of around 3 are likely to adsorb to soils and sludge, commonly used over-the-counter medicines [77], and highly prescribed pharmaceuticals, where prescriptions dispensed have increased over successive years [25,78]. Compared to recognised environmental preparative methods for wastewater and solid samples, the QuEChERS approach potentially offers a reduction in preparation time, from hours to 20 minutes per sample and reduced extraction cost, estimated at $63 \%$ for the extraction cartridges alone, therefore, it is prudent to investigate the potential of the QuEChERS method further. We aim to develop a method that offers good recovery of compounds, with minimal matrix interferences, using a reversed-phase liquid chromatography-mass spectrometry (LC-MS) method for multi-residue detection to reliably quantitate these compounds using an internal standard approach. 
Table 1.2: Summary data for the suite of compounds chosen for this study, based upon the compounds of interest to CIP and WFD, and previous in-house data.

\begin{tabular}{|c|c|}
\hline Compounds & Structure \\
\hline $\begin{array}{l}\text { Acetaminophen } \\
\text { Formula: } \mathbf{C}_{8} \mathbf{H}_{9} \mathbf{N O}_{2} \\
\text { pKa: } 10.2 \\
\text { logP: } 0.34 \\
\text { Chemistry: Basic } \\
\end{array}$ & \\
\hline $\begin{array}{l}\text { Citalopram Hydrobromide } \\
\text { Formula: } \mathbf{C}_{20} \mathbf{H}_{21} \mathbf{F N}_{2} \mathbf{O} \\
\text { pKa: } 9.4 \\
\text { logP: } 2.51 \\
\text { Chemistry: Basic }\end{array}$ & \\
\hline $\begin{array}{l}\text { Carbamazepine } \\
\text { Formula: } \mathbf{C}_{15} \mathbf{H}_{12} \mathbf{N}_{2} \mathbf{O} \\
\text { pKa: } 14.3 \\
\text { logP: } 2.67 \\
\text { Chemistry: Basic }\end{array}$ & \\
\hline $\begin{array}{l}\text { Erythromycin } \\
\text { Formula: } \mathbf{C}_{37} \mathbf{H}_{67} \mathbf{N O}_{13} \\
\text { pKa: } 8.6 \\
\text { logP: } 2.83 \\
\text { Chemistry: Basic }\end{array}$ & \\
\hline $\begin{array}{l}\text { Propranolol Hydrochloride } \\
\text { Formula: } \mathbf{C}_{16} \mathbf{H}_{21} \mathbf{N O}_{2} \\
\text { pKa: } 9.5 \\
\text { logP: } 3.1 \\
\text { Chemistry: Basic }\end{array}$ & \\
\hline $\begin{array}{l}\text { Diphenhydramine Hydrochloride } \\
\text { Formula: } \mathbf{C}_{17} \mathbf{H}_{21} \mathbf{N O} \\
\text { pKa: } 8.7 \\
\text { logP: } 3.66 \\
\text { Chemistry: Basic }\end{array}$ & \\
\hline $\begin{array}{l}\text { Ibuprofen } \\
\text { Formula: } \mathbf{C}_{13} \mathbf{H}_{18} \mathbf{O}_{2} \\
\text { pKa: } 4.3 \\
\text { logP: } 3.72 \\
\text { Chemistry: Acidic } \\
\end{array}$ & \\
\hline $\begin{array}{l}\text { Diclofenac Sodium } \\
\text { Formula: } \mathbf{C}_{14} \mathbf{H}_{11} \mathbf{C l}_{2} \mathbf{N O}_{2} \\
\text { pKa: } 4.4 \\
\text { logP: } 4.06 \\
\text { Chemistry: Acidic }\end{array}$ & \\
\hline
\end{tabular}




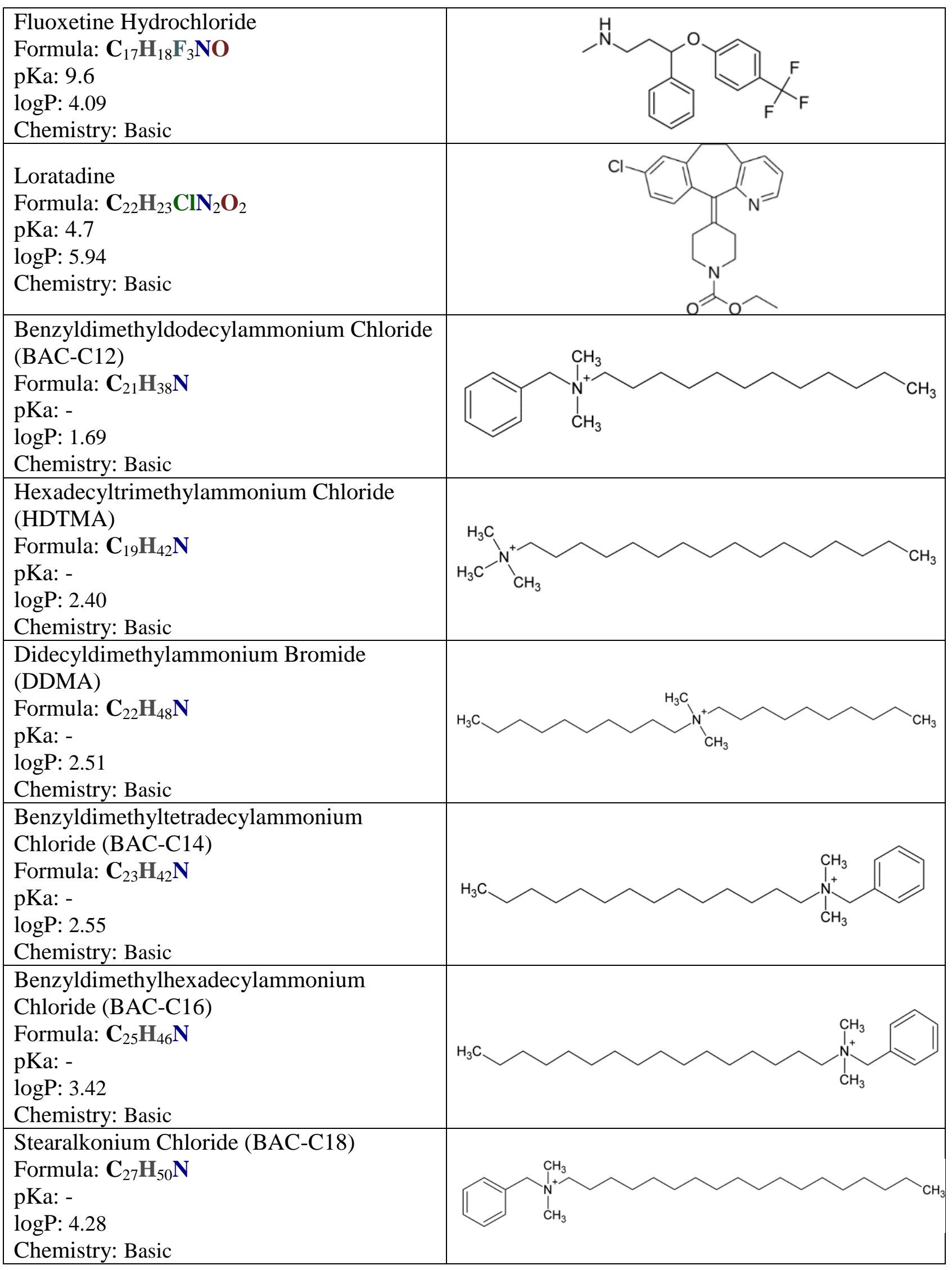




\section{7: Research Aims}

The overall aim of this thesis was to develop a QuEChERS-based sample preparation method suitable for the extraction of acidic and basic compounds, including a selection of pharmaceuticals and biocides of interest to the Chemical Investigation Programme. This development was undertaken to ensure minimal matrix effects and maximum compound recovery were achieved from complex environmental matrices, including wastewater effluent, treated sludgecake and locally sourced biota, with qualitative and quantitative investigations achieved using an LC-MS approach. The specific objectives were to:

1. To develop a liquid chromatography-mass spectrometry (LC-MS) qualitative and quantitative analytical platform for a suite of pharmaceuticals, with a particular focus on highly prescribed and common over-the-counter medicines, and commonly used biocides that may contribute to informing CIP II and WFD.

2. To investigate and benchmark the QuEChERS sample preparation technique for the chosen compounds versus current recognised methods used by regulatory agencies (e.g. EPA and Natural Resources Wales (NRW)) for wastewater treatment samples (i.e. treated effluent and sludgecake) and chosen species of biota (molluscs).

3. Qualitative mass spectrometry investigation of wastewater treatment samples (i.e. treated effluent and sludgecake) and chosen species of biota (molluscs) for compounds of interest to CIP II and WFD.

4. Quantitative mass spectrometry investigation of wastewater treatment samples (i.e. treated effluent and sludgecake) and molluscs for the selected compounds of interest. 


\section{References}

[1] Gómez MJ, Martínez Bueno MJ, Lacorte S, Fernández-Alba AR, Agüera A. Pilot survey monitoring pharmaceuticals and related compounds in a sewage treatment plant located on the Mediterranean coast. Chemosphere. 2007; 66: 993-1002.

[2] Davies J, Schumacher M, Gazzard D. The determination of ofloxacin, oxytetracycline, propranolol, erythromycin and fluoxetine in sludge by liquid chromatography tandem triple quadrupole mass spectrometry using online SPE enrichment. Environment Agency Method of Analysis Suite One - Sludge. 2010: 1-12.

[3] Environmental Protection Agency. Pharmaceuticals and personal care products in water, soil, sediment and biosolids by HPLC/MS/MS. Washington, USA: EPA; 2007. Method 1694.

[4] EPA website: Ozone pollution. Health effects of ozone pollution. Available from: https://www.epa.gov/ozone-pollution/health-effects-ozone-pollution

[5] European Communities Council Directive of 21 May 1991 concerning urban waste water treatment (91/271/EEC). Aberdeen: Official Journal of the European Communities; 1991. Report No.: L135/40.

[6] Department for Environment Food and Rural Affair (DEFRA). Waste water treatment in the United Kingdom - 2012. DEFRA. 2012. Report No.: PB13811.

[7] Water UK. Wastewater treatment and recycling. London: Water UK; 2006.

[8] Thames Water. The sewage treatment process [Internet]. Reading, UK: Thames Water. Available from: http://www.thameswater.co.uk/cycles/accessible/sewage treatment.html

[9] World Bank Organisation. Sanitation, Hygiene and Wastewater Resource Guide - Introduction to wastewater treatment processes [Internet]. Washington, USA: World Bank. Available from: http://water.worldbank.org/shw-resource-guide/infrastructure/menu-technicaloptions/wastewater-treatment

[10] Statutory Instruments. The sludge (use in agriculture) regulation 1989. Public Health, England and Wales and Public Health, Scotland; 1989. Report No.: 1263.

[11] ADAS. The safe sludge matrix: guidelines for the application of sewage sludge to agricultural land. England: ADAS; 2001.

[12] European Union. Directive 2000/60/EC of the European Parliament and of the council of 23 October 2000. Aberdeen: Official Journal of the European Union; 2000. Report No.: L327.

[13] European Union. Directive 2008/105/EC of the European Parliament and of the Council of 16 December 2008. Aberdeen: Official Journal of the European Union; 2008. Report No.: L348/84.

[14] Gardner M. The UKWIR Chemical Investigations Programme - a mid-programme update. London: UK Water Industry Research (UKWIR); 2012. 
[15] European Union. Directive 2013/39/EU of the European Parliament and of the council of 12 August 2013. Aberdeen: Official Journal of the European Union; 2013. Report No.: L226.

[16] UK Water Industry Research. The Chemical Investigations Programme Phase 2, 2015-2020 Introduction. Available from: https://www.ukwir.org/the-chemicals-investigation-programmephase-2,-2015-2020

[17] Department for Environment, Food and Rural Affairs. (DEFRA). National Implementation Plan for the Stockholm Convention on Persistent Organic Pollutants. 2007. DEFRA. 2007. Report No.: PB11971.

[18] World Health Organisation. http://www.who.int/foodsafety/areas_work/chemical-risks/pops/en/

[19] Prakash V, Bishwakarma MC, Chaudhary A, Cuthbert R, Dave R, Kulkarni M, Kumar S, Paudel K, Ranade S, Shringarpure R, Green RE. The population decline of Gyps Vultures in India and Nepal has slowed since veterinary use of diclofenac was banned. PLoS ONE. 2012; 7(11): e49118.

[20] Galligan TH, Amano T, Prakash VM, Kulkarni M, Shringarpure R, Prakash N, Ranade S, Green RE, Cuthbert RJ. Have population declines in Egyptian Vulture and Red-headed Vulture in India slowed since the 2006 ban on veterinary diclofenac? Bird Conservation International. 2014: 1-10.

[21] Kidd KA, Blanchfield PJ, Mills KH, Palace VP, Evans RE, Lazorchak JM, Flick RW. Collapse of a fish population after exposure to a synthetic estrogen. Proceedings of the National Academy of Sciences (PNAS). 2007; 104 (21): 8897-8901.

[22] Anderson SE, Meade BJ. Potential Health Effects Associated with Dermal Exposure to Occupational Chemicals. Environmental Health Insights. 2014; 8 (Suppl. 1): 51-62.

[23] Hahn S, Schneider K, Gartiser S, Heger W, Mangelsdorf I. Consumer exposure to biocides identification of relevant sources and evaluation of possible health effect. Environmental Health, 2010; 9:7.

[24] Schweer C. Biocides - risks and alternatives. Challenges and perspectives regarding the handling of biocides in the EU. PAN Germany. 2010.

[25] Welsh Government, Statistics for Wales, Prescriptions dispensed in the community in Wales, 2016. 24 May 2017, SFR 56/2017.

[26] Scotland and Northern Ireland Forum for Environmental Research (SNIFFER). Methodology for the analysis of selected pharmaceuticals and drugs of abuse in sediments and sludge. Edinburgh; 2009. Report No.: ER09.

[27] Halling-Sorensen B, Nors Nielsen S, Lanzky PF, Ingerslev F, Holten Lutzheft HC, Jorgensen SE. Occurrence, fate and effects of pharmaceutical substances in the environment - a review. Chemosphere, 1998. 36(2): 357-393. 
[28] White DG, McDermott PF. Biocides, drug resistance and microbial evolution. Current Opinion in Microbiology. 2001; 4: 313-317.

[29] Scientific Committee on Emerging and Newly Identified Health Risks. Assessment of the Antibiotic Resistance Effects of Biocides; 2009.

[30] Berna J.L, Ferrer J, Moreno A, Prats D, Ruiz Bevia F. The fate of LAS in the environment. Tenside Surfactants Detergents. 1989; 26: 101-107.

[31] Prats D, Ruiz F, Vazquez B, Rodríguez-Pastor M. Removal of Anionic and Nonionic Surfactants in a Wastewater Treatment Plant with Anaerobic Digestion. A Comparative Study. Water Research. 1997; 31: 1925-1930.

[32] Gray MA, Peake SJ, Farrell AP, Bruch R. Acute Didecyl Dimethyl Ammonium Chloride Toxicity to Larval Lake Sturgeon, Acipenser fulvescens Rafinesque, Walleye Sander vitreus Mitchill, and Northern Pike, Esox lucius Linnaeus. Bulletin of Environmental Contamination and Toxicology. 2005 Nov; 75(5):890-896.

[33] Traverso-Soto JM, González-Mazo E, Lara-Martín PA. Analysis of Surfactants in Environmental Samples by Chromatographic Techniques. In: Chromatography - The Most Versatile Method of Chemical Analysis. InTech; 2012.

[34] McBain AJ, Ledder RG, Moore LE, Catrenich CE, Gilbert P. Effects of QuaternaryAmmonium-Based Formulations on Bacterial Community Dynamics and Antimicrobial Susceptibility. Applied and Environmental Microbiology. 2004; 70(6): 3449-3456.

[35] Littlejohn TG, Paulsen IT, Gillespie MT, Tennent JM, Midgley M, Jones IG, Purewal AS, Skurray RA. Substrate specificity and energetics of antiseptic and disinfectant resistance in Staphylococcus aureus. FEMS Microbiology Letters. 1992; 74:259-265.

[36] Rouch, DA, Cram DS, DiBerardino D, Littlejohn TG, Skurray RA. Efflux-mediated antiseptic resistance gene qacA from Staphylococcusaureus: common ancestry with tetracycline- and sugar-transport proteins. Molecular Microbiology. 1990; 4:2051-2062.

[37] Gerba CP. Quaternary Ammonium Biocides: Efficacy in Application. Applied and Environmental Microbiology. 2015; 81(2):464-469.

[38] Wales AD, Davies RH. Co-Selection of Resistance to Antibiotics, Biocides and Heavy Metals, and its Relevance to Foodborne Pathogens. Antibiotics. 2015; 4: 567-604.

[39] Sargent M. Guide to achieving reliable quantitative LC-MS measurements. RSC Analytical Methods Committee; 2013.

[40] Kachhawaha AS, Nagarnaik1 PM, Jadhav M, Pudale A, Labhasetwar PK, Banerjee K. Optimization of a Modified QuEChERS Method for Multiresidue Analysis of Pharmaceuticals and Personal Care Products in Sewage and Surface Water by LC-MS/MS. Journal of AOAC International. 2017; 100 (3): 592-597. 
[41] Cerqueira MBR, Guilherme JR, Caldas SS, Martins ML, Zanella R, Primel EG. Evaluation of the QuEChERS method for the extraction of pharmaceuticals and personal care products from drinking-water treatment sludge with determination by UPLC-ESI-MS/MS. Chemosphere. 2014; 107: 74-82.

[42] Townsend R, Desbrow C, Godfrey AR. Extraction of over-the-counter pharmaceuticals from water and sludge cake using ISOLUTE® QuEChERS prior to LC/MS analysis. Biotage, 2014. Report No.: AN831 Available from: http://www.biotage.com/news/clean-up-ofpharmaceuticals-in-sludge-cake

[43] Langford A, Dean J, Reed R, Holmes D, Weyers J, Jones A. Practical skills in forensic science. England: Pearson Education Limited; 2005.

[44] Mitra S. Sample preparation techniques in analytical chemistry. New Jersey: John Wiley \& Sons Inc.; 2003.

[45] Anastassiades M, Lehotay SJ, Stajnbaher D, Schenck FJ. Fast and easy multiresidue method employing acetonitrile extraction/partition and "dispersive solid-phase extraction" for the determination of pesticide residues in produce. Journal of AOAC International. 2003; 86 (2): 412-431.

[46] AOAC Official Method 2007.01 Pesticide Residues in Foods by Acetonitrile Extraction and Partitioning with Magnesium Sulfate. AOAC International.

[47] Foods of plant origin - Determination of pesticide residues using GC-MS and/or LC-MS/MS following acetonitrile extraction/ partitioning and cleanup by dispersive SPE - QuEChERS method. Brussels: European Committee for Standardization; 2008. Report No.: BS EN 15662:2008.

[48] Mohan C. Buffers: A guide for the preparation and use of buffers in biological systems. Germany: Calbiochem, EMD Biosciences Inc.; 2003.

[49] Lehotay SJ, de Kok A, Hiemstra M, van Bodegraven P. Validation of a fast and easy method for the determination of residues from 229 pesticides in fruits and vegetables using gas and liquid chromatography and mass spectrometric detection. Journal of AOAC International. 2005; 88: 595-614.

[50] Bragança I, Plácido A, Paíga P, Domingues VF, Delerue-Matos C. QuEChERS: A new sample preparation approach for the determination of ibuprofen and its metabolites in soils. Science of the Total Environment. 2012; 433: 281-289.

[51] Berlioz-Barbier A, Vauchez A, Wiest L, Baudot R, Vulliet E, Cren-Olivé C. Multi-residue analysis of emerging pollutants in sediment using QuEChERS-based extraction followed by LC-MS/MS analysis. Analytical and Bioanalytical Chemistry. 2014 Feb; 406(4):1259-1266. 
[52] Dankyi E, Gordon C, Carboo D, Fomsgaard IS. Quantification of neonicotinoid insecticide residues in soils from cocoa plantations using a QuEChERS extraction procedure and LCMS/MS. Science of The Total Environment. 2014 Nov; 499:276-283.

[53] Boyd RK, Basic C, Bethem RA. Trace quantitative analysis by mass spectrometry. England: John Wiley \& Sons Ltd.; 2008.

[54] Chromacademy. The Theory of HPLC - Band Broadening. Crawford Scientific.

[55] de Hoffmann E, Stroobant V. Mass spectrometry, principles and applications. 3rd Edition. England: John Wiley \& Sons Ltd.; 2011.

[56] Fenn JB, Mann M, Meng CK, Wong SF. Electrospray ionization - principles and practice. Mass Spectrometry Reviews. 1990; 9: 37-70.

[57] Wilm M. Principles of Electrospray Ionization. Molecular Cell Proteomics. 2011; 10(7): Published online $19^{\text {th }}$ May 2011.

[58] Touboul D, Jecklin MC, Zenobi R. Ion internal energy distributions validate the charge residual model for small molecule ion formation by spray methods. Rapid Communications in Mass Spectrometry. 2008; 22: 1062-1068.

[59] Dass C. Fundamentals of Contemporary Mass Spectrometry. 1st Edition. USA: John Wiley \& Sons Ltd.; 2007.

[60] March RE. An introduction to quadrupole ion trap mass spectrometry. Journal of Mass Spectrometry. 1997; 32: 351-369.

[61] Cooks GR, Kaiser RE. Quadrupole ion trap mass spectrometry. Accounts of Chemical Research. 1990; 23 (7): 213-219.

[62] Thermo Scientific. LTQ Orbitrap Operator's Manual. European Training Institute.

[63] Zubarev RA, Makarov A. Orbitrap Mass Spectrometry. Analytical Chemistry. 2013; 85: $5288-5296$.

[64] Chernushevich IV, Loboda AV, Thomson BA. An Introduction to quadrupole-time-of-flight mass spectrometry. Journal of Mass Spectrometry. 2001; 36: 849-865.

[65] Balogh MP. The Mass Spectrometry Primer. Waters Inc.2009.

[66] Gracia-Lor E, Sancho JV, Hernández F. Multi-class determination of around 50 pharmaceuticals, including 26 antibiotics, in environmental and wastewater samples by ultrahigh performance liquid chromatography-tandem mass spectrometry. Journal of Chromatography A. 2011; 1218: 2264-2275.

[67] López-Serna R, Petrović M, Barceló D. Development of a fast instrumental method for the analysis of pharmaceuticals in environmental and wastewaters based on ultra-high performance liquid chromatography (UHPLC)-tandem mass spectrometry (MS/MS). Chemosphere. 2011; 85: 1390-1399. 
[68] Gros M, Rodríguez-Mozaz S, Barceló D. Fast and comprehensive multi-residue analysis of a broad range of human and veterinary pharmaceuticals and some of their metabolites in surface and treated waters by ultra-high-performance liquid chromatography coupled to quadrupolelinear ion trap mass spectrometry. Journal of Chromatography A.2012; 1248: 104-121.

[69] Luque-Muñoz A, Vílchez JL, Zafra-Gómez A. Multiclass method for the determination of pharmaceuticals and personal care products in compost from sewage sludge using ultrasound and salt-assisted liquid-liquid extraction followed by ultrahigh performance liquid chromatography-tandem mass spectrometry analysis. Journal of Chromatography A. 2017; 1507: 72-83.

[70] Ivanová L, Mackulak T, Grabic R, Golovko O, Koba O, Staňová AV, Szabová P, Grenčíková A, Bodík I. Pharmaceuticals and illicit drugs - A new threat to the application of sewage sludge in agriculture. Science of the Total Environment. 2018; 634: 606-615.

[71] Martínez-Carballo E, González-Barreiro C, Sitka A, Kreuzinger N, Scharf S, Gans O. Determination of selected quaternary ammonium compounds by liquid chromatography with mass spectrometry. Part II. Application to sediment and sludge samples in Austria. Environmental Pollution. 2007; 146: 543-547.

[72] Ruan T, Song S, Wang T, Liu R, Lin Y, Jiang G. Identification and composition of emerging quaternary ammonium compounds in municipal sewage sludge in China. Environmental Science and Technology. 2014; 48: 4289-4297.

[73] Östman M, Lindberg RH, Fick J, Björn, E, Tysklind M. Screening of biocides, metals and antibiotics in Swedish sewage sludge and wastewater. Water Research. 2017; 115: 318-328.

[74] McEneff G, Barron L, Kelleher B, Paull B, Quinn B. The determination of pharmaceutical residues in cooked and uncooked marine bivalves using pressurised liquid extraction, solidphase extraction and liquid chromatography-tandem mass spectrometry. Analytical Bioanalytical Chemistry. 2013; 405: 9509-9521.

[75] Perelló G, Martí-Cid R, Castell V, Llobet JM, Domingo JL. Concentrations of polybrominated diphenyl ethers, hexachlorobenzene and polycyclic aromatic hydrocarbons in various foodstuffs before and after cooking. Food and Chemical Toxicology. 2009; 47: 709-715.

[76] Kalogeropoulos N, Karavoltsos S, Sakellari A, Avramidou S, Dassenakis M, Scoullos M. Heavy metals in raw, fried and grilled Mediterranean finfish and shellfish. Food and Chemical Toxicology. 2012; 50: 3702-3708.

[77] Connelly D. The OTC market in Britain in 2015. The Pharmaceutical Journal. 2016; 296: No. 7887.

[78] Welsh Government, Statistics for Wales, Prescriptions dispensed in the community in Wales, 2015. 18 May 2016, SFR 60/2016. 


\section{Chapter 2: Materials and Methods}

\section{1: Laboratory Equipment}

- $10 \mu \mathrm{L}, 100 \mu \mathrm{L}, 1 \mathrm{~mL}, 10 \mathrm{~mL}$ Transferpette air displacement pipettes

- Mettler Toledo EL204 analytical balance (4 decimal places)

- Fisherbrand FB15012 vortex mixer 2x classic

- Eppendorf centrifuge 5810R

- Scanvac Lyophilser

- Techne Sample Concentrator

- SPE Vacuum Box

- Desiccator

\section{2: Chemicals and Consumables}

\subsection{1: Chemicals}

A suite of 10 pharmaceuticals and 6 quaternary ammonium biocides (QACs) of interest to the Chemical Investigation Programme (CIP) were chosen, based on compounds seen in a qualitative screen as part of previous in-house study. In order to be able to quantify the target pharmaceuticals, surrogate internal standards (IS) were sourced. Unfortunately, due to the high cost of isotopically labelled analogues of most of the target compounds, more pragmatic alternatives were sourced. The IS chosen are drugs that did not pass the pre-clinical studies during drug development or not marketed and therefore should have not been emitted into the environment. Talopram hydrochloride and pronethalol hydrochloride are structural analogues of citalopram and propranolol; these were investigated to determine whether they could be used as an IS and for their scope to be broadened to include for citalopram, diphenhydramine, fluoxetine and diclofenac, and propranolol, respectively due to similarities in structure and retention times. For carbamazepine, a synthetic impurity, 10,11-dihydrocarbamazepine, was chosen and due to similar physio-chemical properties in $\log \mathrm{P}$ and structure this was also tested as an IS for erythromycin and loratadine. For acetaminophen, the smallest compound in the suite, a deuterated analogue was sourced as the physio-chemical properties differed significantly from the rest of the suite. For the biocides (BAC-C12-C18, DDMA and HDTMA), a single IS was chosen for all 6 compounds; the deuterated analogue of BAC-C14, benzyldimethyltetradecylammonium chloride- $\mathrm{d}_{7}\left(\right.$ BAC-C14- $\left.\mathrm{d}_{7}\right)$ due to the similarity in molecular structures and retention time. 
The chemicals and standards used are listed in Table 2.1.

Table 2.1: List of chemicals and pharmaceuticals used and their grade i.e. pharmaceutical secondary standard (PSS), CAS number and the supplier details.

\begin{tabular}{|c|c|c|c|}
\hline Chemical & CAS N $\mathbf{N}^{\mathbf{0}}$ & Grade & $\begin{array}{c}\text { Supplier } \\
\text { Fisher Scientific } \\
\text { (Loughborough, } \\
\text { England) }\end{array}$ \\
\hline Acetonitrile & $75-05-8$ & HPLC & \multirow{3}{*}{$\begin{array}{c}\text { Fisher Scientific } \\
\text { (Loughborough, } \\
\text { England) }\end{array}$} \\
\hline Water & $7732-18-5$ & HPLC & \\
\hline Formic acid & $64-18-6$ & $99.44 \%$ & \\
\hline Acetaminophen & $103-90-2$ & PSS & \multirow{9}{*}{$\begin{array}{l}\text { Sigma Aldrich (Dorset, } \\
\text { England) }\end{array}$} \\
\hline Carbamazepine & $298-46-4$ & PSS & \\
\hline Diclofenac & $15307-79-6$ & PSS & \\
\hline Diphenhydramine Hydrochloride & $147-24-0$ & $\geq 98.0 \%$ & \\
\hline Erythromycin & $114-07-8$ & PSS & \\
\hline Fluoxetine Hydrochloride & $56296-78-7$ & PSS & \\
\hline Ibuprofen & $15687-27-1$ & PSS & \\
\hline Loratadine & $79794-75-5$ & PSS & \\
\hline Propranolol Hydrochloride & $318-98-9$ & PSS & \\
\hline Citalopram Hydrobromide & $59729-32-7$ & $>99.0 \%$ & $\begin{array}{l}\text { Tocris (Abingdon, } \\
\text { England) }\end{array}$ \\
\hline $\begin{array}{l}\text { Benzyldimethyldodecylammonium } \\
\text { Chloride (BAC-C12) }\end{array}$ & $139-07-1$ & $>99.0 \%$ & \\
\hline $\begin{array}{l}\text { Benzyldimethyltetradecylammonium } \\
\text { Chloride (BAC-C14) }\end{array}$ & $139-08-2$ & $>99.0 \%$ & \\
\hline $\begin{array}{l}\text { Benzyldimethylhexadecylammonium } \\
\text { Chloride (BAC-C16) }\end{array}$ & $122-08-9$ & $\mathrm{n} / \mathrm{a}$ & Sigma Aldrich \\
\hline $\begin{array}{l}\text { Didecyldimethylammonium } \\
\text { Bromide (DDMA) }\end{array}$ & $2390-68-3$ & $98 \%$ & \\
\hline $\begin{array}{l}\text { Hexadecyltrimethylammonium } \\
\text { Chloride (HDTMA) }\end{array}$ & $112-02-7$ & $>98 \%$ & \\
\hline Stearalkonium chloride (BAC-C18) & $122-19-0$ & $\mathrm{n} / \mathrm{a}$ & $\begin{array}{c}\text { LGC Standards } \\
\text { (Teddington, England) }\end{array}$ \\
\hline \multicolumn{4}{|l|}{ Internal Standards } \\
\hline Acetaminophen-(methyl)- $\mathrm{d}_{3}$ & $60902-28-5$ & $\mathrm{n} / \mathrm{a}$ & \multirow{2}{*}{ Sigma Aldrich } \\
\hline 10,11-Dihydrocarbamazepine & $3564-73-6$ & $99.0 \%$ & \\
\hline Pronethalol Hydrochloride & $51-02-5$ & $>99.0 \%$ & \multirow{2}{*}{ Tocris } \\
\hline Talopram Hydrochloride & 7013-41-4 & $>99.0 \%$ & \\
\hline $\begin{array}{l}\text { Benzyldimethyltetradecylammonium } \\
\text { chloride- } \mathrm{d}_{7}\left(\text { BAC-C14-d } \mathrm{d}_{7}\right)\end{array}$ & $\begin{array}{c}1219178- \\
72-9\end{array}$ & $\mathrm{n} / \mathrm{a}$ & $\begin{array}{c}\text { Toronto Research } \\
\text { Chemicals (Ontario, } \\
\text { Canada) }\end{array}$ \\
\hline
\end{tabular}




\subsection{2: Consumables}

- $2 \mathrm{~mL}$ and $4 \mathrm{~mL}$ amber-glass Chromacol Ltd. vials

- $20 \mathrm{~mL}$ disposable scintillation vials

- Glass Wheaton and Duran bottles

- $15 \mathrm{~mL}$ and $50 \mathrm{~mL}$ Corning Centristar centrifuge tubes

- $20 \mathrm{~mL}$ Oasis HLB cartridges

\subsection{3: Sample Preparation Consumables}

The following sample preparation consumables were supplied by Biotage EU (Uppsala, Sweden) and Biotage GB (Ystrad Mynach, Wales).

- 6 mL ISOLUTE® ENV+ SPE cartridges

- 6 mL ISOLUTE® SCX-2 SPE cartridges

- $3 \mathrm{~mL}, 6 \mathrm{~mL}$ and $10 \mathrm{~mL}$ custom SPE cartridges containing $\mathrm{MgSO}_{4}$ and PSA

- QuEChERS extraction tubes, detailed in Table 2.2 below

Table 2.2: List of QuEChERS kits used in this project and their chemical composition.

\begin{tabular}{|c|c|c|c|c|c|c|c|c|}
\hline & \multicolumn{8}{|c|}{ Composition } \\
\hline $\begin{array}{l}\text { QuEChERS } \\
\text { Consumable }\end{array}$ & $\mathrm{MgSO}_{4}$ & $P S A$ & C18 & $G C B$ & $\begin{array}{c}\mathrm{Na} \\
\text { Acetate }\end{array}$ & $\mathrm{NaCl}$ & $\begin{array}{c}\mathrm{Na} \\
\text { Citrate }\end{array}$ & $\begin{array}{c}\text { Na Citrate } \\
\text { sesqui- } \\
\text { hydrate }\end{array}$ \\
\hline Custom Extraction Tube & $4 \mathrm{~g}$ & & & & $1.5 \mathrm{~g}$ & & & \\
\hline $\begin{array}{c}\text { AOAC Extraction Tube } \\
(\mathrm{Q} 0010-15 \mathrm{~V})\end{array}$ & $6 \mathrm{~g}$ & & & & $1.5 \mathrm{~g}$ & & & \\
\hline $\begin{array}{c}\text { EN Extraction Tube } \\
(\mathrm{Q} 0020-15 \mathrm{~V})\end{array}$ & $4 \mathrm{~g}$ & & & & & $1 \mathrm{~g}$ & $1 \mathrm{~g}$ & $0.5 \mathrm{~g}$ \\
\hline $\begin{array}{l}\text { AOAC Fruit and Vegetable } \\
\text { (F\&V) Kit (Q0030-15V) }\end{array}$ & $1200 \mathrm{mg}$ & $400 \mathrm{mg}$ & & & & & & \\
\hline $\begin{array}{l}\text { EN Fruit and Vegetable } \\
(\mathrm{F} \& \mathrm{~V}) \text { Kit }(\mathrm{Q} 0035-15 \mathrm{~V})\end{array}$ & $900 \mathrm{mg}$ & $150 \mathrm{mg}$ & & & & & & \\
\hline $\begin{array}{c}\text { AOAC Waxed F\&V Kit } \\
(\mathrm{Q} 0050-15 \mathrm{~V})\end{array}$ & $1200 \mathrm{mg}$ & $400 \mathrm{mg}$ & $400 \mathrm{mg}$ & & & & & \\
\hline $\begin{array}{c}\text { EN Waxed F\&V Kit } \\
(\mathrm{Q} 0060-15 \mathrm{~V})\end{array}$ & $900 \mathrm{mg}$ & $150 \mathrm{mg}$ & $150 \mathrm{mg}$ & & & & & \\
\hline $\begin{array}{c}\text { EN Pigmented F\&V Kit } \\
(\mathrm{Q} 0080-15 \mathrm{~V})\end{array}$ & $900 \mathrm{mg}$ & $150 \mathrm{mg}$ & & $15 \mathrm{mg}$ & & & & \\
\hline $\begin{array}{c}\text { EN Highly Pigmented F\&V } \\
\text { Kit (Q0090-15V) }\end{array}$ & $900 \mathrm{mg}$ & $150 \mathrm{mg}$ & & $45 \mathrm{mg}$ & & & & \\
\hline
\end{tabular}




\section{3: Instrumentation}

\subsection{1: Liquid Chromatography System}

Thermo Finnigan LC system consisting of a Micro AS autosampler and MSPump Plus was used throughout this study and interfaced to mass spectrometry for detection. A confirmatory qualitative screen was performed using a Dionex Ulitmate 300, however LC conditions remained the same.

\subsubsection{1: Liquid Chromatography Columns}

Various LC columns were investigated throughout the method development stage, as described below:

- Waters Xbridge C18 column (1.0 x 100 mm ID, $3.5 \mu \mathrm{m})$ Waters Xselect charged surface hybrid (CSH) C18 column (2.1 x $150 \mathrm{~mm}$ ID, $3.5 \mu \mathrm{m})$

- Waters Xselect high strength silica (HSS) T3 column (1.0 x 100 mm ID, $3.5 \mu \mathrm{m})$

A Phenomenex KrudKatcher Ultra 0.5 micron in-line filter was used or the final assessment of these columns in preparation for complex samples. This was chosen in place of a guard cartridge of the same stationary phases due to the unavailability of this product at this column ID.

\subsubsection{2: Liquid Chromatography Solvents}

Various compositions of mobile phases were investigated during method development, including changing the organic modifier and additives. Optimum conditions (good chromatographic peak shape and reproducible chromatography) were observed using the following solutions:

Mobile Phase A: $0.1 \%$ formic acid in water - The mobile phase was prepared by measuring $500 \mathrm{~mL}$ of HPLC grade water into a $1 \mathrm{~L}$ Wheaton bottle. $500 \mu \mathrm{L}$ was removed using a pipette and $500 \mu \mathrm{L}$ of formic acid was added. The solution was mixed thoroughly before use.

Mobile Phase B: $100 \%$ acetonitrile.

Injector Wash: $0.1 \%$ formic acid in a mixture of $75 \%$ water: $25 \%$ acetonitrile $-150 \mathrm{~mL}$ of HPLC grade water and $50 \mathrm{~mL}$ of HPLC grade acetonitrile were mixed together into a $250 \mathrm{~mL}$ 
Duran bottle. $200 \mu \mathrm{L}$ of the solution was removed replaced with the same volume of formic acid. The solution was mixed thoroughly before use.

These were the final conditions used for characterising the LC-MS method and investigating the quantitative performance as no carryover of the compounds was observed and remained the same for both chromatographic columns and both mass spectrometric systems.

\subsubsection{3: Injector Conditions}

The autosampler was maintained at $4^{\circ} \mathrm{C}$ during operation to prevent solvent evaporation. Each sample $(5 \mu \mathrm{L})$ was injected onto the column via a full loop injection $(20 \mu \mathrm{L})$ to ensure an accurate and reproducible injection volume. The injection needle was washed with $1 \mathrm{~mL}$ of wash solution to prevent carryover of target analytes and the syringe flushed with $4.8 \mu \mathrm{L}$ of wash solution, twice the volume of the syringe, as part of the wash programme to ensure optimum performance of the syringe.

\subsubsection{4: Flow Conditions}

A mobile phase flow rate of $50 \mu \mathrm{L} /$ minute was used throughout analysis and used a gradient elution by increasing to $100 \% \mathrm{~B}$ at the rate of 3.4 percent/minute. The elution method also comprised a post-gradient wash and a re-conditioning step to ensure column conditions were reproducible for each injection.

- Gradient elution: Initial 95\% A: $5 \% \mathrm{~B}$, hold for 2 minutes

Linear ramp to $100 \% \mathrm{~B}$ in 28 minutes and hold for 10 minutes

Linear ramp to initial conditions in 1 minute and hold for 10 minutes

Total run time: 51 minutes

\subsection{2: Mass Spectrometry Analysis}

Final quantitation of pharmaceuticals was undertaken using a Waters Micromass ZQ4000 single quadrupole mass spectrometer, equipped with an electrospray ionisation (ESI) source. A simultaneous qualitative pharmaceutical screen and quantitative analysis of biocides was carried out using a Thermo Finnigan LCQ Classic 3D ion trap mass spectrometer with ESI source. The LCQ was operated in positive ionisation mode only. A confirmatory qualitative screen was performed on a Thermo Scientific LTQ Orbitrap XL mass spectrometer to obtain 
accurate mass data to aid identification of any compounds of interest. As with the LCQ, this was operated in positive ionisation mode only.

\subsubsection{1: Quantitation Method (ZQ4000)}

Prior to LC-MS method development the mass spectrometer was calibrated and tuned for the target precursor ion of the pharmaceutical and internal standard. This involved optimising the cone voltage to maximise the precursor ion signal observed without inducing in-source fragmentation. The source and desolvation settings used were in accordance with the manufacturer specifications for a flow rate of $50 \mu \mathrm{L} /$ minute.

Table 2.3: This table shows the Waters ZQ4000 electrospray (ESI) source operation settings used for this study. The cone voltage for each compound was set within the instrument method.

\begin{tabular}{|l|c|c|}
\hline \multicolumn{1}{|c|}{ Setting } & Positive Mode & Negative Mode \\
\hline Capillary Voltage $(\mathrm{kV})$ & 3.50 & 2.81 \\
\hline Cone Voltage $(\mathrm{V})$ & $15($ Variable) & 15 (Variable) \\
\hline RF Lens $(\mathrm{V})$ & 0.5 & 0.5 \\
\hline Source Temperature $\left({ }^{\circ} \mathrm{C}\right)$ & 80 & 120 \\
\hline Desolvation Temperature $\left({ }^{\circ} \mathrm{C}\right)$ & 120 & 100 \\
\hline Desolvation Gas Flow $(\mathrm{L} / \mathrm{hr})$ & 250 & 250 \\
\hline Cone Gas Flow $(\mathrm{L} / \mathrm{hr})$ & 50 & 50 \\
\hline
\end{tabular}

The mass spectrometer was operated in full mass scan mode over a mass-to-charge $(\mathrm{m} / \mathrm{z})$ range of 125-775, and selected ion monitoring (SIM) mode for the relevant precursor masses in positive and negative ionisation conditions. Data was acquired using MassLynx software in positive ion mode and was captured in continuum for a complete profile of the ions. As quantitative LC-MS requires at least 10 data points across the chromatographic peak, the LCMS method was segmented into individual SIM scans, according to each compound's retention time. A full mass scan in positive and negative ionisation mode was recorded over the entire chromatographic run (51 minutes) to generate a comprehensive chromatographic profile for each sample. Data was processed using QuanLynx using a Savitzky-Golay smoothing factor of 1 and a peak threshold of 1.5 and 2.0 for height and area, respectively. As part of the quantitative analysis, manual integration was required; this was performed by using the automatic peak integration function in the chromatogram window within MassLynx software. The peak area was then manually divided by the automated peak area of the extracted ion chromatogram of the corresponding internal standard to generate the relative 
response factor (RRF), which was inputted into the regression equation to determine concentration (see Eq 2.9). Statistical analysis was carried out using Microsoft Excel.

Table 2.4: A summary of the optimised cone voltages used for the SIM scan for each compound. Each compound had a scan time of 0.4 seconds and an inter-scan delay of 0.01 seconds, with the exception of ibuprofen (analysed in negative mode), which had an inter-scan delay of 0.1 seconds.

\begin{tabular}{|l|c|}
\hline Compound & Cone Voltage (V) \\
\hline Acetaminophen & 20 \\
\hline Acetaminophen-(methyl)- $\mathrm{d}_{3}$ & 25 \\
\hline Carbamazepine & 20 \\
\hline Citalopram & 20 \\
\hline Diclofenac & 10 \\
\hline Diphenhydramine & 10 \\
\hline Erythromycin & 10 \\
\hline Fluoxetine & 15 \\
\hline Ibuprofen & 15 \\
\hline Loratadine & 10 \\
\hline Pronethalol & 5 \\
\hline Propranolol & 25 \\
\hline Talopram & 15 \\
\hline 10,11-Dihydrocarbamazepine & 35 \\
\hline
\end{tabular}

\subsubsection{2: Pharmaceutical Screen and Biocide Quantitation Method (LCQ)}

Prior to method development the mass spectrometer was calibrated and tuned using a specific calibration mixture containing caffeine, MRFA and Ultramark to optimise the capillary voltage $(3 \mathrm{~V})$, tube lens offset $(10 \mathrm{~V})$ and source voltage $(4.5 \mathrm{kV})$. The sheath gas flow of 60 and capillary temperature of $200^{\circ} \mathrm{C}$ were set in accordance to the manufacturer's specification for a flow rate of $50 \mu \mathrm{L} /$ minute. The mass spectrometer was operated in full mass scan mode over a $\mathrm{m} / \mathrm{z}$ range of $100-800$, with product ion scans recorded for target pharmaceutical masses and selected reaction monitoring (SRM) mode for the biocides, with the exception of HDTMA which was recorded as a SIM scan, in positive ionisation conditions.

For quantifying the target biocides a pilot study carried out in-house determined the optimal collision energies for each compound, and these parameters were used in this method. Data was acquired using Xcalibur software in positive ion mode and was captured in continuum for a complete profile of the ions. As quantitative LC-MS requires at least 10 data points across the chromatographic peak, the LC-MS method was partitioned into four segments and 
the scan events divided across the segments to minimize the duty cycle. Each segment of the method recorded a full mass scan to generate a comprehensive chromatographic profile for each sample. Data processing was carried out using QualBrowser and QuanBrowser with statistical analysis performed using Microsoft Excel.

Table 2.5: A summary of the processing and integration settings used to quantitate the biocides using QuanBrowser. All data was processed from the SRM/SIM data with peaks integrated providing the signal-tonoise (S/N) is above 3, using ICIS peak detection algorithm, a Savitzky-Golay smoothing function of 3 with a baseline window of 40 and noise factors of 5 and 10 for area and peak, respectively.

\begin{tabular}{|c|c|c|c|c|c|c|}
\hline \multirow[b]{2}{*}{ Compound } & \multirow[b]{2}{*}{$\begin{array}{l}\text { Scan } \\
\text { Type }\end{array}$} & \multirow[b]{2}{*}{ Scan Filter } & \multicolumn{4}{|c|}{ Segments } \\
\hline & & & $\begin{array}{c}1 \\
(0-5 \\
\text { min) }\end{array}$ & $\begin{array}{c}2 \\
(15-20 \\
\text { min) }\end{array}$ & $\begin{array}{c}3 \\
(20-26 \\
\text { min) }\end{array}$ & $\begin{array}{c}4 \\
(26-51 \\
\text { min) }\end{array}$ \\
\hline All & FMS ms & {$[100.00-800.00]$} & $\checkmark$ & $\checkmark$ & $\checkmark$ & $\checkmark$ \\
\hline Acetaminophen & FMS ms2 & $152.00 @ 35.00[50.00-200.00]$ & $\checkmark$ & & & \\
\hline Acet-d $\mathrm{d}_{3}$ & FMS ms2 & 155.00@35.00 [50.00-200.00] & $\checkmark$ & & & \\
\hline Pronethalol & FMS ms2 & $230.00 @ 40.00$ [60.00-250.00] & $\checkmark$ & & & \\
\hline Propranolol & FMS ms2 & $260.00 @ 40.00$ [70.00-275.00] & $\checkmark$ & $\checkmark$ & & \\
\hline Diphenhydramine & FMS ms2 & $256.00 @ 30.00[70.00-275.00]$ & & $\checkmark$ & & \\
\hline Citalopram & FMS ms2 & $325.00 @ 40.00$ [85.00-350.00] & & $\checkmark$ & & \\
\hline Erythromycin & FMS ms2 & $734.00 @ 40.00$ [200.00-750.00] & & $\checkmark$ & & \\
\hline Carbamazepine & FMS ms2 & $237.00 @ 40.00$ [65.00-250.00] & & $\checkmark$ & & \\
\hline $10,11-\mathrm{DHC}$ & FMS ms2 & $239.00 @ 40.00$ [65.00-250.00] & & $\checkmark$ & & \\
\hline Fluoxetine & FMS ms2 & $310.00 @ 40.00$ [85.00-350.00] & & $\checkmark$ & & \\
\hline Talopram & FMS ms2 & $296.00 @ 40.00$ [80.00-325.00] & & $\checkmark$ & & \\
\hline Loratadine & FMS ms2 & $383.00 @ 40.00$ [105.00-400.00] & & $\checkmark$ & $\checkmark$ & \\
\hline Diclofenac & FMS ms2 & $296.00 @ 30.00[80.00-325.00]$ & & & $\checkmark$ & \\
\hline BAC-C12 & SRM ms2 & $304.00 @ 40.00$ [211.00-213.00] & & & $\checkmark$ & $\checkmark$ \\
\hline BAC-C14 & SRM ms2 & $332.00 @ 42.00$ [239.00-241.00] & & & & $\checkmark$ \\
\hline BAC-C16 & SRM ms2 & $360.00 @ 44.00$ [267.00-269.00] & & & & $\checkmark$ \\
\hline BAC-C18 & SRM ms2 & $388.00 @ 48.00$ [295.00-297.00] & & & & $\checkmark$ \\
\hline$\overline{\text { DDMA }}$ & SRM ms2 & $326.00 @ 48.00$ [185.00-187.00] & & & & $\checkmark$ \\
\hline HDTMA & SIM ms & {$[283.00-285.00]$} & & & & $\checkmark$ \\
\hline BAC-C14-d 7 & SRM ms2 & 339.00@40.00 [239.00-241.00] & & & & $\checkmark$ \\
\hline
\end{tabular}

\subsubsection{3: Qualitative Screen (LTQ Orbitrap)}

Similar to the LCQ, this mass spectrometer was tuned and calibrated prior to analysis using a mixture of caffeine, MRFA and Ultramark to optimise the capillary voltage, tube lens offset and source voltage, and an optimal sheath gas flow of 25 and capillary temperature of $275^{\circ} \mathrm{C}$ were used for this analysis. The mass spectrometer was operated in positive ionisation mode and data was recorded using a full mass scan over a $m / z$ range of 100-1000 for 51 minutes, with an additional DDA scan set to fragment the most intense parent ion detected in the pre- 
scan using a fixed collision energy of $40 \mathrm{~V}$ to generate accurate mass data. Data was analysed using QualBrowser in Xcalibur 3.0.

Table 2.6: The optimum electrospray source settings for the LTQ Orbitrap used for the qualitative screen.

\begin{tabular}{|l|c|}
\hline \multicolumn{1}{|c|}{ Setting } & Positive Mode \\
\hline Capillary Voltage $(\mathrm{V})$ & 40 \\
\hline Capillary Temperature $\left({ }^{\circ} \mathrm{C}\right)$ & 275 \\
\hline Tube Lens Offset $(\mathrm{V})$ & 130 \\
\hline Source Voltage $(\mathrm{kV})$ & 3.6 \\
\hline Sheath Gas Flow (arbitrary units) & 25 \\
\hline
\end{tabular}

\section{4: Solutions}

\subsection{1: Standard Stock Solutions}

$1 \mathrm{mg} / \mathrm{mL}$ standard stock solution: $1 \mathrm{mg}$ of material was weighed into an amber glass chromacol vial and $1 \mathrm{~mL}$ of HPLC grade water or acetonitrile was added, followed by vortexing to ensure material was completely dissolved. Where possible, solutions were prepared in water to limit degradation or evaporative effects, with the exception of the biocides, ibuprofen, erythromycin, loratadine, carbamazepine and 10,11dihydrocarbamazepine which were made up in $100 \%$ acetonitrile, due to limited solubility at this concentration.

\subsection{2: Working Solutions}

These were used as a sub-stock for the calibration graph, and made as an analyte mixture by adding an appropriate amount of the stock solutions to a diluent of 50:50 acetonitrile/water. All solutions were vortexed before use.

$1 \mu \mathrm{g} / \mathrm{mL}$ working solution: $1 \mu \mathrm{L}$ of each standard stock solution was dispensed into an amber glass chromacol vial containing the appropriate amount of 50:50 HPLC grade water/HPLC grade acetonitrile solvent mixture. The sample was vortexed before use and an additional mixture prepared for the internal standard working solution.

\subsection{3: Calibration and Quality Control (QC) Samples}

Calibration graphs were produced for each compound using eleven calibration standards of increasing concentration, made using a $1 \mu \mathrm{g} / \mathrm{mL}$ working solution. Each graph was plotted 
using regression statistics as relative response ratio versus concentration, and the ability to quantitate was characterised using the Xselect HSS T3 columns.

Table 2.7: The concentration range and corresponding volume used for each pharmaceutical calibration standard and quality control (QC) standards when analysed using the Xselect H33 T3. SB consisted of 50:50 acetonitrile/water and the S0 contained $100 \mathrm{ng} / \mathrm{mL}$ of internal standard mixture only.

\begin{tabular}{|l|c|c|c|c|c|c|c|c|c|}
\hline \multicolumn{10}{|c|}{ Calibration Standards for Pharmaceutical Analysis using Xselect HSS T3 } \\
\hline Standard & S1 & S2 & S3 & S4 & S5 & S6 & S7 & S8 & S9 \\
\hline Concentration (ng/mL) & 1 & 5 & 10 & 25 & 50 & 100 & 200 & 300 & 400 \\
\hline
\end{tabular}

\begin{tabular}{|l|c|c|c|c|}
\hline \multicolumn{5}{|c|}{ Quality Control (QC) Samples for Pharmaceutical Analysis using Xselect HSS T3 } \\
\hline Standard & V. Low & Low & Mid & High \\
\hline Concentration (ng/mL) & 15 & 25 & 100 & 350 \\
\hline
\end{tabular}

For biocide measurement, an alternative calibration and QC range were used to accommodate the difference in compound sensitivity and expected relative abundance.

Table 2.8: The concentration range and corresponding volume used for each biocide calibration standard and quality control (QC) standards when analysed using the Xselect H33 T3. SB consisted of 50:50 acetonitrile/water and the $\mathrm{S} 0$ contained $20 \mathrm{ng} / \mathrm{mL}$ of internal standard mixture only.

\begin{tabular}{|l|c|c|c|c|c|c|c|c|}
\hline \multicolumn{10}{|c|}{ Calibration Standards for Biocide Analysis using Xselect HSS T3 } \\
\hline Standard & S1 & S2 & S3 & S4 & S5 & S6 & S7 & S8 \\
\hline Concentration $(\mathbf{n g} / \mathbf{m L})$ & 2 & 6 & 10 & 20 & 30 & 50 & 70 & 80 \\
\hline
\end{tabular}

\begin{tabular}{|l|c|c|c|c|}
\hline \multicolumn{5}{|c|}{ Quality Control (QC) Samples for Biocide Analysis using Xselect HSS T3 } \\
\hline Standard & V. Low & Low & Mid & High \\
\hline Concentration (ng/mL) & 8 & 20 & 60 & 80 \\
\hline
\end{tabular}

\subsection{4: Sample Preparation Working Solutions}

Several working solutions were prepared in $2 \mathrm{~mL}$ and $4 \mathrm{~mL}$ amber glass chromacol vials. Each working solution was prepared from a $1 \mu \mathrm{g} / \mathrm{mL}$ dilution of the stock solutions to a concentration of $200 \mathrm{ng} / \mathrm{mL}$ for both the pharmaceuticals and associated IS, and $60 \mathrm{ng} / \mathrm{mL}$ and $20 \mathrm{ng} / \mathrm{mL}$ for the biocides and biocides IS, respectively.

1. Pharmaceuticals working solution (P) - $200 \mathbf{n g} / \mathbf{m L}: 200 \mu \mathrm{L}$ of $1 \mu \mathrm{g} / \mathrm{mL}$ in $800 \mu \mathrm{L}$ of 50:50 acetonitrile/water.

2. Pharmaceutical IS working solution (P-IS) - $200 \mathbf{n g} / \mathbf{m L}: 200 \mu \mathrm{L}$ of $1 \mu \mathrm{g} / \mathrm{mL}$ in $800 \mu \mathrm{L}$ of 50:50 acetonitrile/water.

3. Biocides working solution (B) $-\mathbf{6 0} \mathbf{n g} / \mathbf{m L}$ : $60 \mu \mathrm{L}$ of $1 \mu \mathrm{g} / \mathrm{mL}$ in $940 \mu \mathrm{L}$ of 50:50 acetonitrile/water. 
4. Biocide IS working solution (B-IS) - $20 \mathbf{n g} / \mathbf{m L}$ : $20 \mu \mathrm{L}$ of $1 \mu \mathrm{g} / \mathrm{mL}$ in $980 \mu \mathrm{L}$ of 50:50 acetonitrile/water.

5. QuEChERS spiking solution - Target analytes only: $400 \mu \mathrm{L}$ of $\mathrm{P}+240 \mu \mathrm{L}$ of $\mathrm{B}$ in $1360 \mu \mathrm{L}$ of $50: 50$ acetonitrile/water.

6. QuEChERS spiking solution - IS only: $400 \mu \mathrm{L}$ of P-IS $+80 \mu \mathrm{L}$ of B-IS in $1520 \mu \mathrm{L}$ of 50:50 acetonitrile/water.

The 1:400 dilutions for the extraction of biocides in soil and sludge were made using the 1 $\mathrm{mg} / \mathrm{mL}$ stock solutions.

1. QuEChERS 1:400 Biocides only: $48 \mu \mathrm{L}$ of $1 \mathrm{mg} / \mathrm{mL}$ stock solution for each biocide in $712 \mu \mathrm{L}$ of $50: 50$ acetonitrile/water.

2. QuEChERS 1:400 biocide IS only: $16 \mu \mathrm{L}$ of $1 \mathrm{mg} / \mathrm{mL}$ stock solution for each biocide in $984 \mu \mathrm{L}$ of 50:50 acetonitrile/water.

\section{5: Sample Preparation Method}

The sample preparation method used in this study was a based upon the QuEChERS protocol. The method was assessed using spike before extraction (SBE) quality controls, which are spiked with analyte and IS prior to extraction and spike after extraction (SAE) quality controls, spiked with analyte and IS after the extraction. These samples were used to determine the matrix effects and recovery of each compound, as described by Matuszewski et al. [1].

\subsection{1: QuEChERS Extraction}

For method development, QuEChERS extractions were performed in triplicate to obtain precision data for matrix effect and recovery measurements. Various modifications were made (as described in Chapter 5) to the basic QuEChERS workflow as detailed below. 
Table 2.9: A summary of the optimised QuEChERS sample preparation protocol used throughout this study. The modifications made to the method are detailed in Chapter 5.

\begin{tabular}{|c|c|}
\hline \multicolumn{2}{|c|}{$\begin{array}{l}\text { Initial samples made up in water before testing with } 2.5 \mathrm{~g} \text { of soil, sludge or } \\
\text { homogenised biota samples. }\end{array}$} \\
\hline $\begin{array}{c}\text { SBE: } \\
3.5 \mathrm{~mL} \mathrm{H}_{2} \mathrm{O} \\
+500 \mu \mathrm{L} \text { Drug }+ \text { Internal Standards } \\
\end{array}$ & $\begin{array}{c}\text { SAE: } \\
3.5 \mathrm{~mL} \mathrm{H} \mathrm{H}_{2} \mathrm{O} \\
+500 \mu \mathrm{L} 50: 50 \mathrm{ACN} / \mathrm{H}_{2} \mathrm{O} \\
\end{array}$ \\
\hline \multicolumn{2}{|c|}{$\downarrow$} \\
\hline \multicolumn{2}{|c|}{$\begin{array}{l}+10 \mathrm{ml} \text { ACN }+ \text { QuEChERS Extraction Mixture } \\
\left.\text { (4 } \mathrm{g} \mathrm{MgSO}_{4}+1.5 \mathrm{~g} \mathrm{NaOAc}\right) \\
\text { Shake for } 1 \text { minute } \\
\text { Centrifuge @ } 4000 \mathrm{rpm} \text { for } 5 \text { minutes }\left(21^{\circ} \mathrm{C}\right)\end{array}$} \\
\hline \multicolumn{2}{|c|}{$\downarrow$} \\
\hline \multicolumn{2}{|c|}{$\begin{array}{l}\text { Take extract supernatant (upper organic layer) and add to d-SPE tube } \\
\text { EN Fruit and Vegetable d-SPE } \\
(900 \mathrm{mg} \mathrm{MgSO}+150 \mathrm{mg} \text { PSA }) \\
\text { Vortex for } 1 \text { minute } \\
\text { Centrifuge @ } 4000 \text { rpm for } 5 \text { minutes }\end{array}$} \\
\hline \multicolumn{2}{|c|}{$\downarrow$} \\
\hline \multicolumn{2}{|c|}{ Pre-concentration by evaporation under Nitrogen and reconstitution } \\
\hline \multicolumn{2}{|c|}{$\downarrow$} \\
\hline $\begin{array}{l}\text { Reconstitute in } 500 \mu \mathrm{L} \text { of } \\
50: 50 \mathrm{ACN} / \mathrm{H}_{2} \mathrm{O}\end{array}$ & $\begin{array}{l}\text { Reconstitute in } 500 \mu \mathrm{L} \text { of } \\
\text { Drug + Internal Standards }\end{array}$ \\
\hline \multicolumn{2}{|c|}{$\boldsymbol{V}$} \\
\hline \multicolumn{2}{|c|}{$\begin{array}{c}\text { Vortex for } 1 \text { minute } \\
\text { Transfer to a LC chromacol vial for analysis }\end{array}$} \\
\hline
\end{tabular}

\subsubsection{1: Spike Before Extraction Quality Controls}

All SBE samples were prepared in $50 \mathrm{~mL}$ centrifuge tube.

1) For aqueous extractions: $250 \mu \mathrm{L}$ of separate analyte and IS QuEChERS spiking solutions was spiked into $3.5 \mathrm{~mL}$ of water before the QuEChERS extraction protocol was performed.

2) For control soil extractions: $2.5 \mathrm{~g}$ of soil was weighed into a $50 \mathrm{~mL}$ centrifuge tube, before the addition of $3.5 \mathrm{~mL}$ of water. $250 \mu \mathrm{L}$ of separate analyte and IS QuEChERS spiking solutions was then spiked into the sample before the QuEChERS extraction protocol was performed. 


\subsubsection{2: Spike After Extraction Quality Controls}

All SAE samples were prepared in $50 \mathrm{~mL}$ centrifuge tube.

1) For aqueous extractions: Before extraction, $500 \mu \mathrm{L}$ of $50: 50$ acetonitrile/water was spiked into $3.5 \mathrm{~mL}$ of water, replicating the composition of the working solutions. After extraction, the samples were reconstituted in $250 \mu \mathrm{L}$ of separate analyte and IS QuEChERS spiking solutions and vortexed before analysis.

2) For control soil extractions: Before extraction, $500 \mu \mathrm{L}$ of 50:50 acetonitrile/water and $3.5 \mathrm{~mL}$ of water was spiked into $2.5 \mathrm{~g}$ of soil, replicating the composition of the working solutions. After extraction, the samples were reconstituted in $250 \mu \mathrm{L}$ of separate analyte and IS QuEChERS spiking solutions and vortexed before analysis.

3) For the effluent, sludge and biota quantitative samples: IS working solutions only were used during these extractions, whereby the sample was reconstituted in $250 \mu \mathrm{L}$ spike of IS spiking solution and $250 \mu \mathrm{L}$ of 50:50 acetonitrile/water.

\subsection{2: Environmental Protection Agency (EPA), Method 1694 Extraction}

The current method for the extraction of pharmaceuticals from environmental matrices is the United States Environmental Protection Agency (EPA) Method 1694: Pharmaceuticals and Personal Care Products in Water, Soil, Sediment, and Biosolids by HPLC/MS/MS [2]. This is a two-part extraction procedure comprised of an extensive liquid extraction protocol dependant on analyte chemistry, before SPE using HLB cartridges. The SPE section of the method was tested using a water sample spiked with drug and IS before extraction to determine the matrix effects and recovery of the target pharmaceuticals within this study, for comparison with the optimised QuEChERS method. 


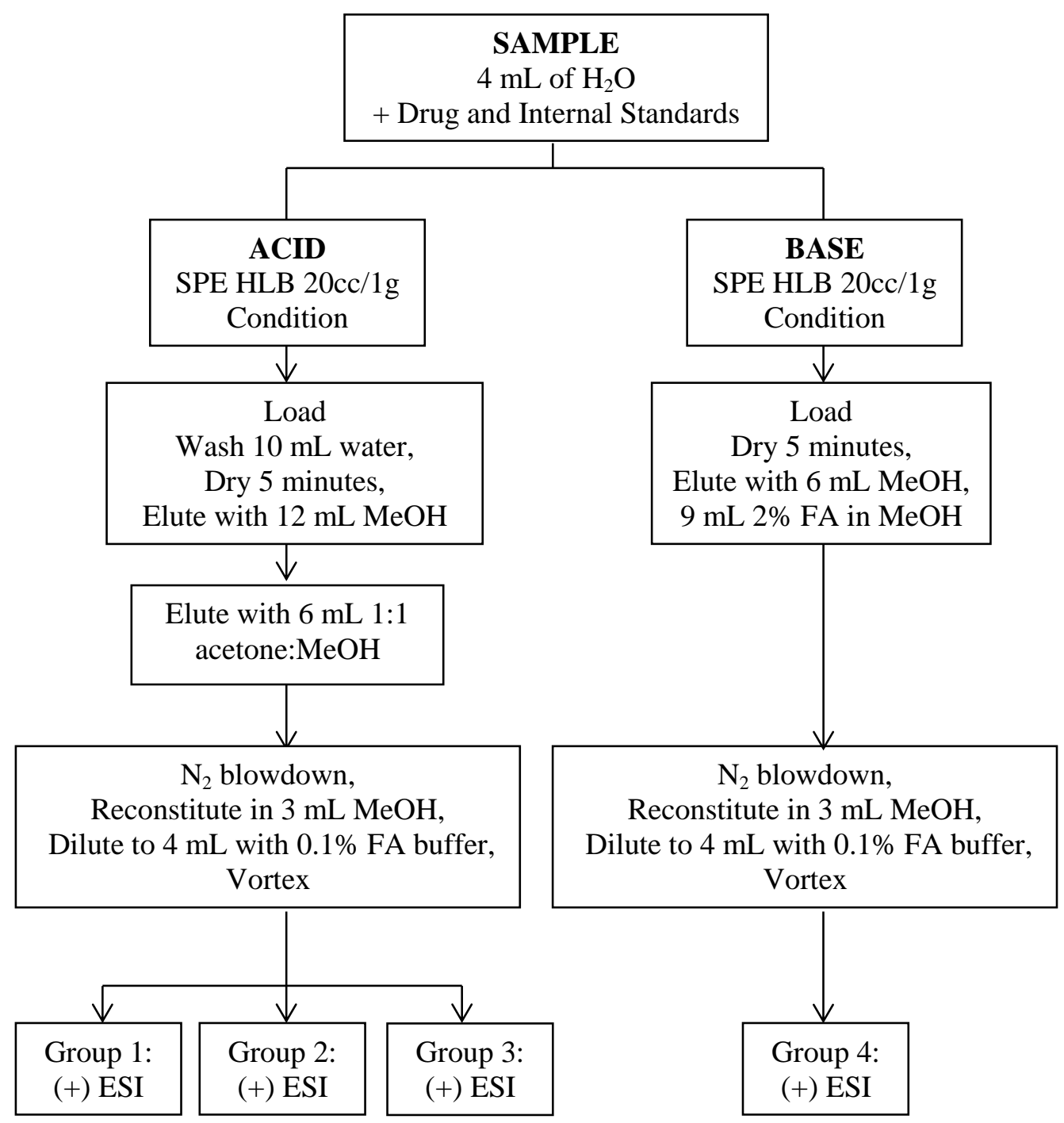

Figure 2.1: The SPE protocol for the EPA Method 1694 detailing the extraction for acidic and basic compounds.

\subsection{3: Natural Resources Wales (NRW) Extraction}

Due to varied performance of EPA method 1694 adaptations have been made by other regulatory agencies, such as Natural Resources Wales, a local environmental monitoring agency that have developed a method for the extraction of a selection of pharmaceuticals from sludgecake [3]. This method was investigated using a soil sample spiked with both drug and IS before extraction to characterise matrix effects and recovery of the target pharmaceuticals and to benchmark the extraction of target compounds from sludgecake against the optimised QuEChERS protocol. 


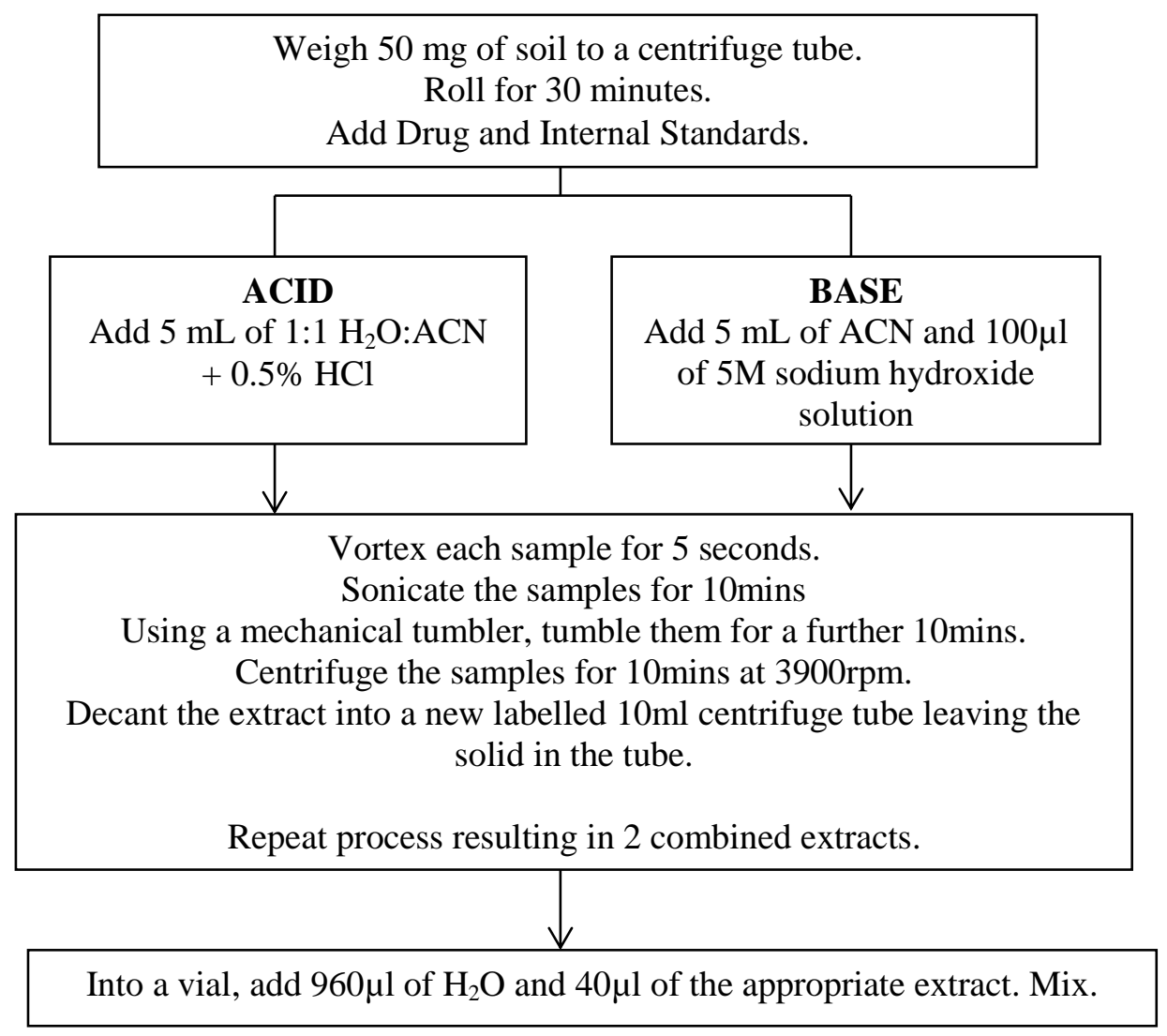

Figure 2.2: The NRW method for the extraction of pharmaceuticals from sludgecake adapted for the analysis of a spiked soil sample, detailing the extraction for acidic and basic compounds.

\section{6: Statistical Analysis}

A number of statistical tests were used to analyse the LC-MS data. These will be described and explained below.

\subsection{1: Relative Standard Deviation (\% RSD)}

Chromatographic repeatability was determined by calculating the relative standard deviation (\%RSD) of the retention time of each drug and IS. This is a measure of the relative error of the method and is the ratio between the mean and the standard deviation of a data set [4]. A value of less than $5 \%$ indicates that the chromatography is repeatable between multiple injections that are performed sequentially.

$$
\begin{aligned}
& \text { Relative Standard Deviation }(\% \mathrm{RSD})=100 \mathrm{~s} / \overline{\mathrm{x}} \\
& \text { Where: } \mathrm{s}=\text { standard deviation } \\
& \bar{x}=\text { mean }
\end{aligned}
$$




\subsection{2: F-Test}

Reproducibility was also determined using sequential injections of the same standard over two days. The variance $\left(\mathrm{s}^{2}\right)$ for each compound for both data sets are statistically analysed using a two-tailed F-test to determine whether a significant difference is observed. The F-test is calculated with the larger variance as the numerator, and the result compared to a critical value. If the result is below the critical value, then there are no significant differences between the data sets, and therefore the chromatography shows good reproducibility over a given time period.

$$
\mathrm{F}=\frac{\mathrm{s}_{1}^{2}}{\mathrm{~s}_{2}^{2}}
$$

\subsection{3: Grubbs’ Test}

A Grubbs' Test was used to determine whether outliers were present within the calculated concentrations for the QC samples. This statistical test compares the deviation of the suspect value from the sample mean, which is divided by the standard deviation of the sample [4]. If the calculated value of $G$ is greater than the critical value, the suspect value in question is rejected as an outlier, and can therefore be left out of the accuracy and precision calculations for the QC set.

$$
\text { Grubbs' Test }(G)=\frac{\mid \text { suspect value }-\bar{x} \mid}{\mathrm{s}}
$$

\subsection{4: Accuracy and Precision}

Accuracy and precision of the calculated concentration of the QC samples were assessed to determine the feasibility of the method for quantitation. Both inter- and intra-day precision was determined using three independent calibration data sets to establish the reliability of the method to measure concentration over multiple experiments. The acceptance criteria used to define good accuracy and precision are $<20 \%$ at the limit of quantitation and $<15 \%$ for the remaining QCs. These figures of merit were determined using the following formulas:

$$
\begin{gathered}
\text { Accuracy }(\%)=\left[\frac{\text { Measured concentration-theoretical concentration }}{\text { Theoretical concentration }}\right] \times 100 \\
\text { Precision }(\%)=\left[\frac{\text { Standard deviation of measured concentration }}{\text { Mean of measured concentration }}\right] \times 100
\end{gathered}
$$




\subsection{5: Instrument Detection Limit (IDL) and Instrument Quantitation Limit (IQL)}

The instrument detection limit (IDL) to determine the lowest discernible signal was calculated using two different methods, statistically and empirically using the following formulas:

$$
\mathrm{IDL}=\frac{3.3 \times \text { Standard error } \text { intercept }_{\text {in }}}{\text { Slope }}
$$

IDL $=3 \times$ Standard deviation of the concentration of the blank

The instrument quantitation limit (IQL) was also determined using two different methods; empirically using the formula below and then confirmed with lowest QC to have good accuracy and precision $(<20 \%)$.

$$
\mathrm{IQL}=10 \mathrm{x} \text { Standard deviation of the concentration of the blank }
$$

\subsection{6: Regression Statistics}

Regression statistics were calculated manually as a weighted regression functions were assessed. The relative response factor for the compounds of interest was determined, and used to form the calibration equation $(y=m x+c)$. This was derived from the following equations, detailed by Almeida et al. [5], where $x$ and $y$ are the RRF and theoretical concentrations of each replicate calibration measurement, respectively, and $\mathrm{w}$ is the weighting factor chosen (i.e. linear $=1$ and weighted $=1 / x$ ).

$$
\begin{aligned}
& \text { Relative Response Factor }(\mathrm{RRF})=\frac{\text { Peak area of analyte }}{\text { Peak area of internal standard }} \\
& \qquad \begin{array}{l}
a=\frac{\sum w_{i} \cdot \sum w_{i} x_{i} y_{i}-\sum w_{i} x_{i} \cdot \sum w_{i} y_{i}}{\sum w_{i} \cdot \sum w_{i} x_{i}^{2}-\left(\sum w_{i} x_{i}\right)^{2}} \\
a=\frac{\sum w_{i} x_{i}^{2} \cdot \sum w_{i} y_{i}-\sum w_{i} x_{i} \cdot \sum w_{i} x_{i} y_{i}}{\sum w_{i} \cdot \sum w_{i} x_{i}^{2}-\left(\sum w_{i} x_{i}\right)^{2}} \\
r=\frac{\sum w_{i} \cdot \sum w_{i} x_{i} y_{i}-\sum w_{i} x_{i} \cdot \sum w_{i} y_{i}}{\sqrt{\sum w_{i} \cdot \sum w_{i} x_{i}^{2}-\left(\sum w_{i} x_{i}\right)^{2}} \cdot \sqrt{\sum w_{i} \cdot \sum w_{i} y_{i}^{2}-\left(\sum w_{i} y_{i}\right)^{2}}}
\end{array}
\end{aligned}
$$




\subsection{7: Heteroscedasticity}

The heteroscedasticity of the data was assessed using the recommendations detailed by Almeida et al. [5]. An F-test of the RRF of the S1 and S9 calibration standards determined whether the variance was statistically different between the high and low end of the calibration line and if the calculated F value exceed F critical $(2,2 ; 0.95=19.00)[4]$, then the response was deemed heteroscedastic. The use of different weighting factors was then tested by the percentage relative error (\%RE) calculated for the replicate measurement of each calibration standard and the weighting factor that gives the smallest sum of absolute relative errors is considered the most appropriate.

$$
\text { Relative Error }(\% \mathrm{RE})=\frac{\text { Calculated value of } \mathrm{x}-\text { Theoretical value of } \mathrm{x}}{\text { Theoretical value of } \mathrm{x}} \times 100
$$

\subsection{8: Extraction Performance}

The performance of the QuEChERS extraction was assessed using the method set out by Matuszewski et al. [1] using the following formulas:

$$
\begin{aligned}
& \text { Matrix Effects }(\%)=\frac{\text { Peak area of spike after extraction }}{\text { Peak area of standard }} \times 100 \\
& \text { Recovery }(\%)=\frac{\text { Peak area of spike before extraction }}{\text { Peak area of spike after extraction }} \times 100
\end{aligned}
$$

Once calculated, these percentages were applied to the peak area of the target analytes detected within the effluent, sludgecake and biota samples to determine the "true" concentration. 


\section{References}

[1] Matuszewski BK Constanzer ML, Chavez-Eng CM. Strategies for the assessment of matrix effect in quantitative bioanalytical methods based on HPLC - MS/MS. Analytical Chemistry. 2003; 75 (13): 3019-3030.

[2] Environmental Protection Agency. Pharmaceuticals and personal care products in water, soil, sediment and biosolids by HPLC/MS/MS. Washington, USA: EPA; 2007. Method 1694.

[3] Davies J, Schumacher M, Gazzard D. The determination of ofloxacin, oxytetracycline, propranolol, erythromycin and fluoxetine in sludge by liquid chromatography tandem triple quadrupole mass spectrometry using online SPE enrichment. Environment Agency Method of Analysis Suite One - Sludge. 2010: 1-12

[4] Miller JN, Miller JC. Statistics and chemometrics for analytical chemistry. Fourth Edition. London: Prentice Hall; 2000.

[5] Almeida AM, Castel-Branco MM, Falcao AC. Linear regression for calibration lines revisited: weighting schemes for bioanalytical methods. Journal of Chromatography B. 2002; 774(2):215-222. 


\section{Chapter 3: Liquid Chromatography-Mass Spectrometry Method Development}

\section{1: MS Detection and Identification of Pharmaceuticals for Quantitation}

The first aspect of LC-MS method development is to ascertain the selectivity of detection, whether the compounds can be detected without interference. This often requires separation or analysis of "pure" samples to determine the precursor and characteristic fragment ions that may be used to qualify the presence of the precursor compound before online LC-MS analysis. Standard solutions were therefore analysed by electrospray ionisation-mass spectrometry (ESI-MS) by both positive and negative ionisation modes as the suite of pharmaceuticals comprised of acidic and basic drugs. Each compound was infused directly into the mass spectrometer at a concentration of approximately $10 \mathrm{pmol} / \mu \mathrm{L}$. The ability to obtain fragmentation data by tandem mass spectrometry (MS/MS) is not available on a single quadrupole mass spectrometer operating with a soft ionisation source such as ESI, however, the instrument chosen for this work does enable some enhancement of selectivity with compound fragmentation by in-source fragmentation. This is achieved by adjusting the voltage within the ESI source (i.e. cone voltage) which excites the precursor ion, causing it to fragment. Increasing the cone voltage sequentially from $5-35 \mathrm{~V}$ was shown to be sufficient to induce fragmentation providing further information to help identify the compound (i.e. a "qualifier" or fragment product ion). The signal-to-noise $(\mathrm{S} / \mathrm{N})$ was calculated over 10 scans using a background signal and the peak intensities of the target compound and any observed product ions to determine which cone voltage $(\mathrm{CV})$ gave the best response for the target compound.

\subsection{1: Acetaminophen}

Acetaminophen has the molecular formula $\mathrm{C}_{8} \mathrm{H}_{9} \mathrm{NO}_{2}$ and a monoisoptopic mass of $151 \mathrm{Da}$. When infused as a standard solution an ion consistent with the protonated molecule ion $[\mathrm{M}+\mathrm{H}]^{+}$is observed at $\mathrm{m} / \mathrm{z}$ 152. Given the elemental composition, the only isotope pattern seen is the ${ }^{13} \mathrm{C}$ as expected at a $1.1 \%$ height of the total number of carbon atoms, i.e. $\mathrm{C}_{8}=$ $8.8 \%{ }^{13} \mathrm{C}$. The fragmentation of acetaminophen in literature reports that the most common product ion is $m / z 110$ [1], and this is observed with increasing cone voltage. This particular fragmentation pattern is the result of a molecular rearrangement of two hydrogen atoms [2] 
and a loss of methanal. This mechanism is confirmed when introducing a deuterated methyl group to the structure as shown in Figure 3.2.

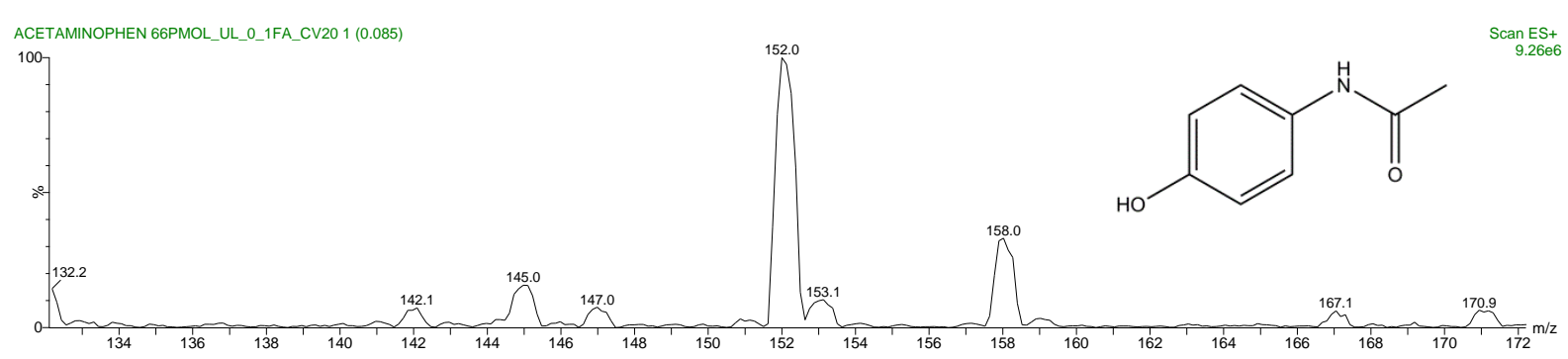

Figure 3.1: The structure of acetaminophen, with the full mass spectrum showing the precursor ion observed at $\mathrm{m} / \mathrm{z} 152$ and the optimum cone voltage (CV) determined by the direct infusion experiments.

\subsection{2: Acetaminophen-(methyl)-d 3}

Similar to acetaminophen (section 3.1.1), acetaminophen-(methyl)- $\mathrm{d}_{3}$ has the molecular formula $\mathrm{C}_{8} \mathrm{H}_{6} \mathrm{D}_{3} \mathrm{NO}_{2}$, where deuterium replace three hydrogen atoms in the methyl group bonded to the carbonyl of the aliphatic section of the structure . This addition results in an increase in mass with the protonated molecule $[\mathrm{M}+\mathrm{H}]^{+}$being observed at $\mathrm{m} / \mathrm{z} 155$. The fragmentation pattern for this compound confirms the hydrogen rearrangement observed with acetaminophen, where the fragment ion showing evidence of only one of the deuterium atoms remaining giving an overall $\mathrm{m} / \mathrm{z} 111$, and the loss of a bi-deuterated methanal neutral molecule of $44 \mathrm{Da}$.

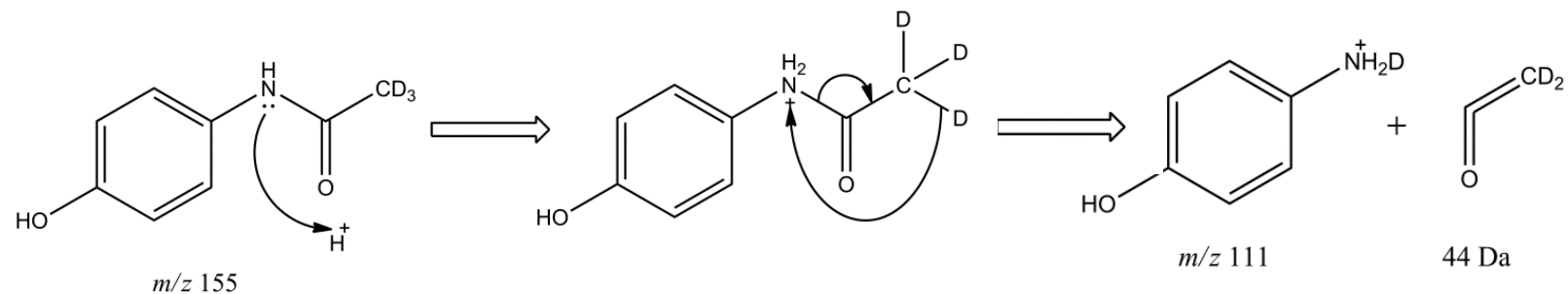

Figure 3.2: The fragmentation mechanism for acetaminophen-(methyl)- $\mathrm{d}_{3}$. The mechanism for acetaminophen would be the same, with the rearrangement of one hydrogen atom from the methyl group to the nitrogen.

\subsection{3: Carbamazepine}

The molecular formula for carbamazepine is $\mathrm{C}_{15} \mathrm{H}_{12} \mathrm{~N}_{2} \mathrm{O}$, which gives a monoisotopic mass of $236 \mathrm{Da}$. The precursor ion observed during direct infusion corresponded to the protonated molecule $[\mathrm{M}+\mathrm{H}]^{+}$at $m / z$ 237. With increasing CV fragmentation was observed at $\mathrm{m} / \mathrm{z} 194$. This fragment ion is consistent with the literature and corresponds with the neutral loss of the 
carbamoyl (CHNO) group [3]. The isotopic peak for ${ }^{13} \mathrm{C}$ can be seen within the spectrum, which is consistent with the $1.1 \%$ height of the total number of carbon atoms (16.5\%), shown in Appendix 3.1.

\subsection{4: Citalopram}

The standard reference material for citalopram is available as a hydrobromide salt $\left(\mathrm{C}_{20} \mathrm{H}_{21} \mathrm{FN}_{2} \mathrm{O}\right.$.HBr$)$, with a monoisotopic mass of $405 \mathrm{Da}$. The precursor ion observed corresponded to the protonated structure of the loss of the hydrobromide salt, $\mathrm{m} / \mathrm{z} 325$ [M$\mathrm{HBr}+\mathrm{H}]^{+}$. This is confirmed in the spectrum by the lack of the distinctive isotope pattern for bromine with data showing evidence of ${ }^{13} \mathrm{C}$ isotope only. The main fragment seen for citalopram was $m / z, 262$, this is proposed to involve the loss of the $\mathrm{C}_{2} \mathrm{H}_{6} \mathrm{~N}$ "tail", and the rearrangement of the carbon chain to form a five carbon ring which results in the loss of the oxygen atom [4].

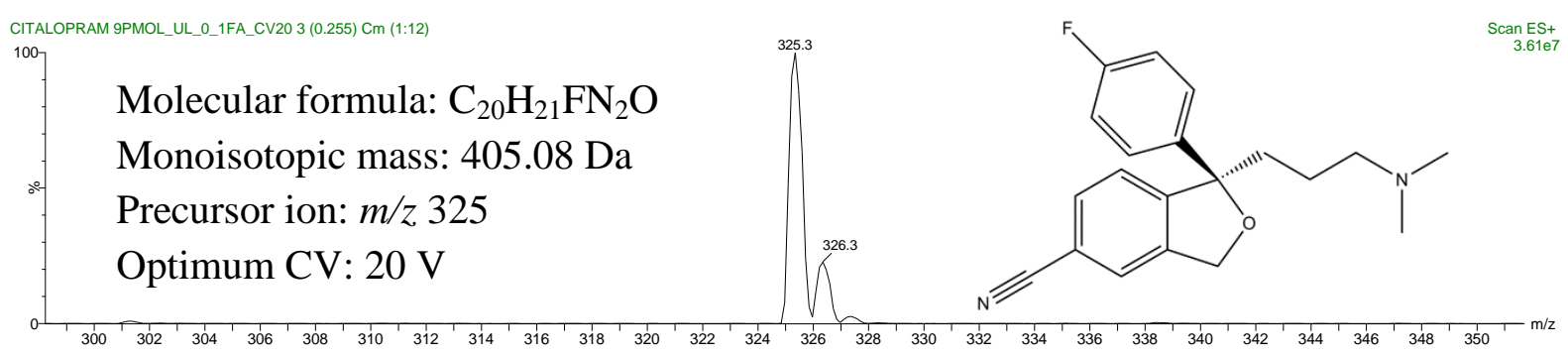

Figure 3.3: The structure of citalopram, with the full mass spectrum showing the precursor ion observed at $m / z$ 325 and the optimum cone voltage $(\mathrm{CV})$ determined by the direct infusion experiments.

\subsection{5: Diclofenac}

Diclofenac is an acidic analyte of pKa 4.4 and is suited to analysis by negative ionisation mode, with the loss of sodium resulting in a negative charge on the adjacent oxygen atom. However, literature and previous in-house studies have showed that it is possible to detect diclofenac in positive ionisation mode with an acidified solution. This is consistent with a substitution of the sodium to a hydrogen atom and the addition of a proton to the nitrogen atom to form ammonium, giving the structure an overall positive charge. While the ZQ4000 mass spectrometer used for this study is capable of running in positive and negative ionisation mode, it was found diclofenac has better signal intensity in positive mode and the precursor ion conditions were tuned and characterised accordingly in this mode. The standard reference material is available as diclofenac sodium salt $\left(\mathrm{C}_{14} \mathrm{H}_{10} \mathrm{Cl}_{2} \mathrm{NNaO}_{2}\right)$ with a monoisotopic mass of $318 \mathrm{Da}$. A precursor ion consistent with the loss of sodium from the 
structure $\left([\mathrm{M}-\mathrm{Na}+\mathrm{H}]^{+}\right)$was observed at $m / z$ 296. Diclofenac has a distinctive isotope pattern due to the presence of two chlorine atoms within the structure; as expected ions consistent with chlorine isotopes at a ratio of $3: 1$ for ${ }^{35} \mathrm{Cl} /{ }^{37} \mathrm{Cl}$, two base units apart [2] were observed at $m / z 298$ and 300. The fragmentation observed were similar to that described in the literature $[5,6]$ with product ions at $\mathrm{m} / \mathrm{z} 278$ and 250 , consistent with the loss of water and the carboxylic acid group, respectively.

\subsection{6: Diphenhydramine}

As with citalopram and diclofenac, the standard reference material for diphenhydramine is available as a hydrochloride salt $\left(\mathrm{C}_{17} \mathrm{H}_{21} \mathrm{NO} . \mathrm{HCl}\right)$. The observed precursor ion is consistent with the loss of the salt $[\mathrm{M}-\mathrm{HCl}+\mathrm{H}]^{+}$, exhibiting an ion at $m / z, 256$, and this did not appear to contain a chlorine isotope pattern, confirming this assumption. Following application of the CV diphenhydramine appears to generate a single product ion at $m / z .167$, indicative of a loss of $89 \mathrm{Da}$, corresponding to the carbon chain from the carbonyl bond [7].

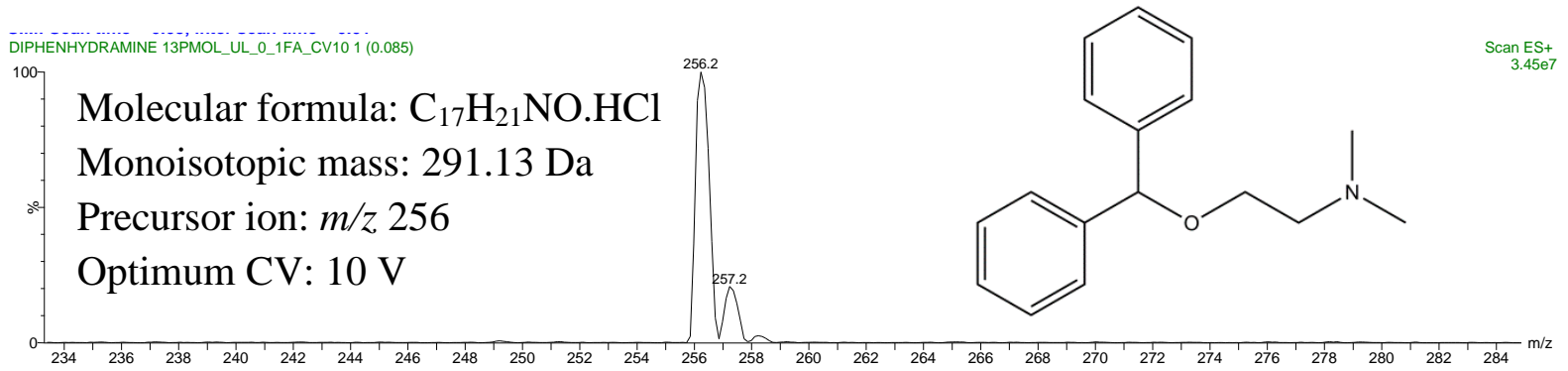

Figure 3.4: The structure of diphenhydramine, with the full mass spectrum showing the precursor ion observed at $m / z 256$ and the optimum cone voltage (CV) determined by the direct infusion experiments.

\subsection{7: Erythromycin}

Erythromycin is a macrolide antibiotic, containing several ring structures resulting in the molecular formula of $\mathrm{C}_{37} \mathrm{H}_{67} \mathrm{NO}_{13}$. The base peak observed during direct infusion at $\mathrm{m} / \mathrm{z} 716$ corresponded to the potential loss of water from this structure and initial characterisation was carried out using this ion. However when injected on column, $\mathrm{m} / \mathrm{z} 716$ was not observed, rather a single peak at $\mathrm{m} / z, 734$ was recorded; the protonated molecular ion $[\mathrm{M}+\mathrm{H}]^{+}$. This is likely due to the increased concentration of acid found in the mobile phase, causing protonation. Given this, $\mathrm{m} / \mathrm{z}, 734$ was assessed in terms of selectivity and adopted as the precursor species for quantitation. The main product ion observed for erythromycin is at $\mathrm{m} / \mathrm{z}$ 
558. This is the same product ion for both $m / z, 734$ and 716, indicative of the sequential loss of water (resulting in the $m / z 716$ ion) and the cladinose sugar ring [8].

\subsection{8: Fluoxetine}

Fluoxetine is supplied as a hydrochloride salt, with the molecular formula $\mathrm{C}_{17} \mathrm{H}_{18} \mathrm{~F}_{3} \mathrm{NO}$. $\mathrm{HCl}$ and monoisotopic weight of $345 \mathrm{Da}$. The loss of $\mathrm{HCl}$ and protonation of the remaining structure gives rise to a precursor ion at $m / z 310$, consistent with $[\mathrm{M}-\mathrm{HCl}+\mathrm{H}]^{+}$. This is clearly observed, along with a product ion at $\mathrm{m} / \mathrm{z}, 148$ when the cone voltage is increased to $15 \mathrm{~V}$, corresponding to the neutral loss of $\mathrm{C}_{7} \mathrm{H}_{5} \mathrm{~F}_{3} \mathrm{O}$ [4] as shown in Figure 3.4. Unlike the other common halogen atoms (i.e. chlorine and bromine) fluorine is a monoisotopic element and therefore does not give an isotope pattern. Therefore, the only isotope expected for fluoxetine is ${ }^{13} \mathrm{C}$, which, and is clearly observed at approximately $18.7 \%$ height of the base peak.<smiles>[Z]COc1ccc(C(F)(F)F)cc1</smiles>

$m / z 310$

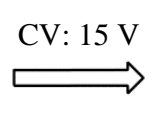

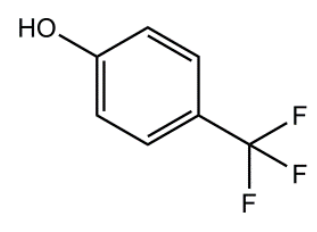

$162 \mathrm{Da}$

Figure 3.5: The fragmentation pattern for fluoxetine showing the precursor ion at $\mathrm{m} / \mathrm{z} 310$ and the proposed fragmentation to produce the observed product ion at $m / z 148$, resulting from the neutral loss of $\mathrm{C}_{7} \mathrm{H}_{5} \mathrm{~F}_{3} \mathrm{O}$.

\subsection{9: Ibuprofen}

Ibuprofen is typically analysed in negative mode, due to the lack of basic groups within the structure and the acidic $\mathrm{pKa}$ of 4.3 . Ionisation occurs through the loss of a proton from the carboxylic acid group resulting in a negatively charged ion. However, for this study, ibuprofen was analysed in both positive and negative mode to see if a signal could be observed similar to diclofenac. The analysis showed negative ionisation mode offered the best sensitivity with the data obtained consistent with a precursor ion of $[\mathrm{M}-\mathrm{H}]^{-}$at $m / z 205$. During direct infusion a product ion was observed at $m / z 161$, corresponding to a potential loss of the carboxylic acid group, supported by the literature [9]. 


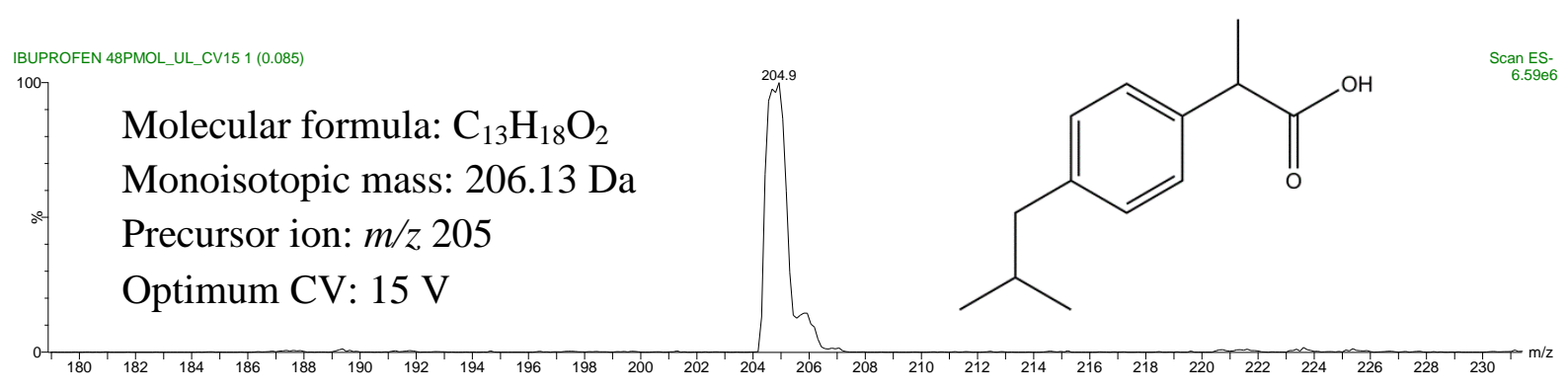

Figure 3.6: The structure of ibuprofen, with the mass spectrum recorded in negative ion mode showing the precursor ion observed at $\mathrm{m} / \mathrm{z}, 205$ and the optimum cone voltage (CV) determined by the direct infusion experiments.

\subsubsection{0: Loratadine}

Loratadine is an antihistamine, with a molecular formula of $\mathrm{C}_{22} \mathrm{H}_{23} \mathrm{~N}_{2} \mathrm{O}_{2} \mathrm{Cl}$ and an ion consistent with a protonated precursor species was observed at $m / z$ 383. From analyses carried out, the protonated molecular ion $[\mathrm{M}+\mathrm{H}]^{+}$at $m / z, 383$ appeared very stable, and insource fragmentation required a high cone voltage of $50 \mathrm{~V}$ to generate a product ion at $\mathrm{m} / \mathrm{z}$ 337. This neutral loss of $46 \mathrm{Da}$ corresponds to the loss of ethanol $\left(\mathrm{C}_{2} \mathrm{H}_{6} \mathrm{O}\right)$ from the bottom of the piperidine ring [10]. There is also a distinctive isotope pattern observed for loratadine consistent with chlorine atoms; a peak at +1 and $+2 \mathrm{~m} / \mathrm{z}$ units of $24.2 \%$ and approximately $40 \%$ of the base peak, indicative of ${ }^{13} \mathrm{C}$ and ${ }^{37} \mathrm{Cl}$ isotopes, respectively (see Figure 3.7).

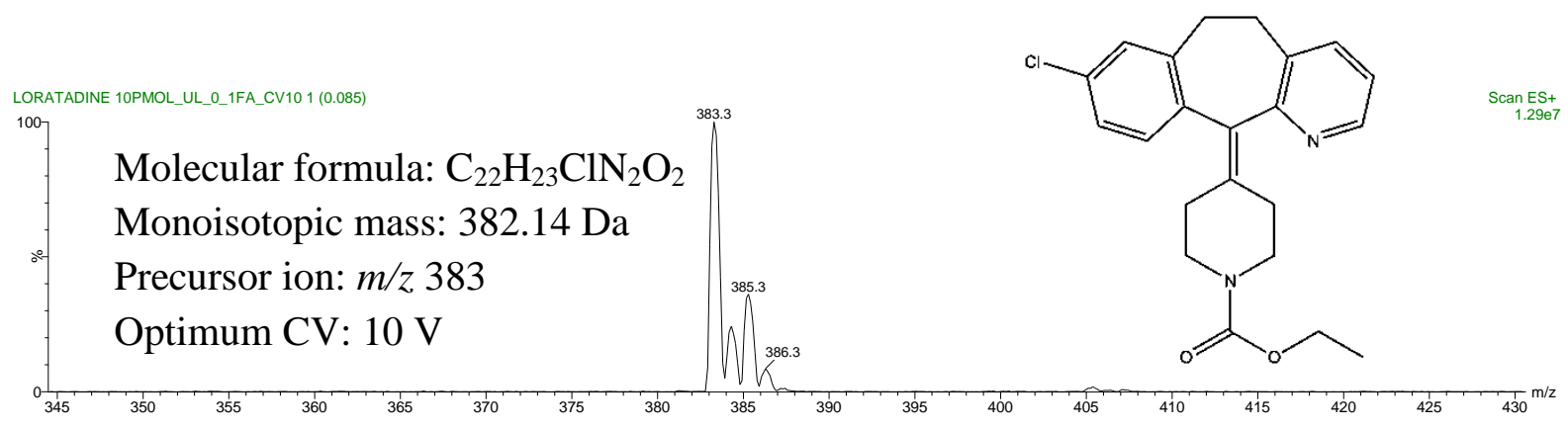

Figure 3.7: The structure of loratadine, with the full mass spectrum showing the precursor ion observed at $m / z$ 383 , with the isotope patterns for ${ }^{13} \mathrm{C}$ and ${ }^{37} \mathrm{Cl}$ and the optimum cone voltage (CV) determined by the direct infusion experiments.

\subsubsection{1: Pronethalol}

Pronethalol is an analogue of propranolol, withdrawn from the clinical market due to its carcinogenicity in mice [11] and is currently sold as a standard in its hydrochloride form. Given this, little is known regarding the fragmentation of pronethalol and so fragmentation mechanisms can only be proposed and not confirmed with corresponding literature. The molecular formula of pronethalol is $\mathrm{C}_{15} \mathrm{H}_{19} \mathrm{NO} . \mathrm{HCl}$, and a precursor ion, observed in positive 
ion mode, at $\mathrm{m} / z 230$ consistent with $[\mathrm{M}-\mathrm{HCl}+\mathrm{H}]^{+}$was recorded. When the cone voltage was increased, another ion at $\mathrm{m} / \mathrm{z} 170$ was observed with increasing intensity; this neutral loss of $60 \mathrm{Da}$ is indicative of a loss of $\mathrm{C}_{3} \mathrm{H}_{8} \mathrm{O}$, resulting from a proposed structural rearrangement of the carbon chain forming an unstable morpholine ring and subsequent fragmentation.<smiles>C=CC(C)NCC(O)c1ccc2ccccc2c1</smiles>

$m / z 230$<smiles>N=CCc1ccc2ccccc2c1</smiles>

$m / z 170$

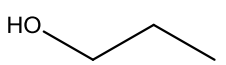

$60 \mathrm{Da}$

Figure 3.8: The fragmentation pattern for pronethalol showing the precursor ion at $\mathrm{m} / \mathrm{z} 230$ and the proposed fragmentation to produce the observed product ion at $m / z, 170$, resulting from the neutral loss of $\mathrm{C}_{3} \mathrm{H}_{8} \mathrm{O}$.

\subsubsection{2: Propranolol}

The standard reference material, propranolol hydrochloride has a molecular formula of $\mathrm{C}_{16} \mathrm{H}_{21} \mathrm{NO}_{2} \cdot \mathrm{HCl}$ and is known to be lost during ionisation with electrospray to form the protonated precursor of $[\mathrm{M}-\mathrm{HCl}+\mathrm{H}]^{+}$at $m / z 260$. This was apparent in the full mass scan and evidence of structural fragmentation was observed with increasing cone voltage with the product ion typically seen for propranolol in studies using collision induced dissociation [12] at $\mathrm{m} / \mathrm{z}$ 183. This product ion is believed to form due to the neutral loss of propylamine $\left(\mathrm{C}_{3} \mathrm{H}_{9} \mathrm{~N}\right)$ and water [13] from the aliphatic part of the structure.

\subsubsection{3: Talopram}

Talopram was initially discovered in 1971 , however it was not commercialised due to a number of suicide attempts during clinical trials [14] and has meant that few available studies have characterised talopram by mass spectrometry. The standard reference material is supplied as a hydrochloride salt, with the molecular formula $\mathrm{C}_{20} \mathrm{H}_{25} \mathrm{NO} . \mathrm{HCl}$ and again, appears to generate a precursor ion consistent with the loss of salt, $[\mathrm{M}-\mathrm{HCl}+\mathrm{H}]^{+}$at $m / z 296$. To assess the MS selectivity of talopram and diclofenac (of sample precursor $\mathrm{m} / \mathrm{z}$ ) the cone voltage was ramped for compound fragmentation. Unfortunately talopram also appeared to show the same or similar fragmentation as diclofenac (loss of water) with an ion observed at $\mathrm{m} / \mathrm{z}$ 278, indicating that chromatographic selectivity and separation would be key in distinguishing these compounds. 


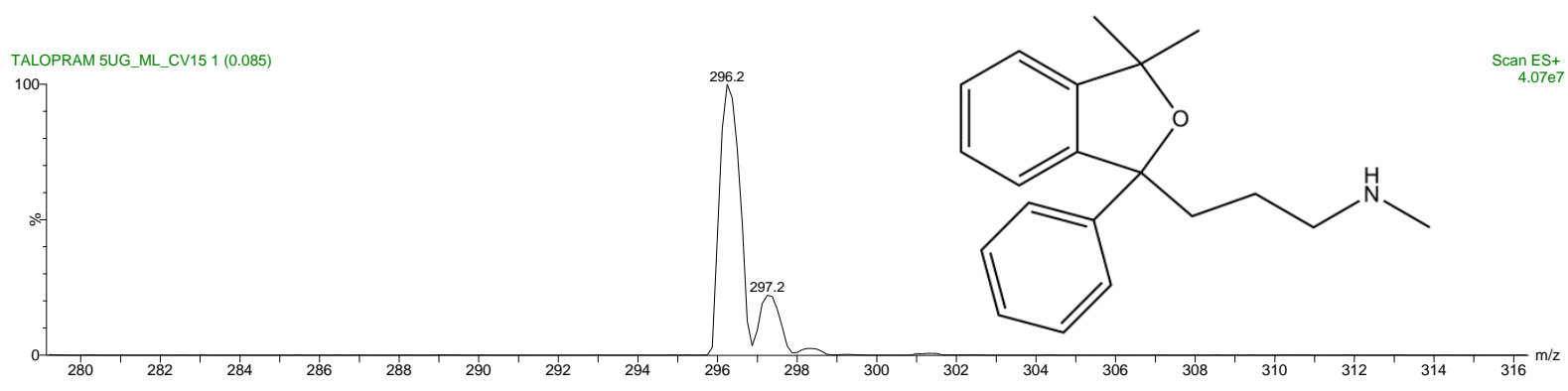

Figure 3.9: The structure of talopram, with the mass spectrum recorded in positive ion mode showing the precursor ion observed at $\mathrm{m} / \mathrm{z}, 205$ and the optimum cone voltage (CV) determined by the direct infusion experiments.

\subsubsection{4: 10,11-Dihydrocarbamazapine}

10,11-Dihydrocarbamazapine is a synthetic impurity of carbamazepine, with a molecular formula of $\mathrm{C}_{15} \mathrm{H}_{14} \mathrm{~N}_{2} \mathrm{O}$. The structural difference between 10,11-DHC and carbamazepine is the absence of the carbon-carbon double bond within the seven-membered ring, and an additional two hydrogen atoms at this position in 10,11-DHC. The precursor ion observed was the protonated molecule consistent with a $[\mathrm{M}+\mathrm{H}]^{+}$, at $m / z$ 239. With increasing cone voltage very little fragmentation was observed with the only product ions identifiable at $\mathrm{m} / \mathrm{z}$ 222 and $\mathrm{m} / \mathrm{z}, 194$ at cone voltage $35 \mathrm{~V}$ and $50 \mathrm{~V}$, respectively. These product ions corresponded to the potential loss of the ammonia $\left[\mathrm{NH}_{3}\right]$ from the amide functional group, and the loss of the entire amide group, leaving a positive charge on the nitrogen atom at the bottom of the seven membered ring, similar to the product ion observed for carbamazepine.<smiles>NC(=O)N1c2ccccc2CCc2ccccc21</smiles>

Figure 3.10: The fragmentation pattern for 10,11-dihydrocarbamazepine showing the precursor ion at $\mathrm{m} / z, 239$ and the proposed fragmentation to produce the observed product ion at $\mathrm{m} / \mathrm{z} 222$, resulting from the neutral loss of $\mathrm{NH}_{3}$ and the product ion at $\mathrm{m} / \mathrm{z} 194$, resulting from the neutral loss of $\mathrm{CH}_{3} \mathrm{NO}$.

\section{2: Development of LC-MS Separation}

The chromatographic separation of the suite of pharmaceutical compounds was characterised using both full mass scan data and individual single ion monitoring (SIM) scans to compare changes in the sensitivity of the analysis for quantitation. For example, the full mass scan can also act as a screen for later analyses using more complex matrices, and is able to capture 
isotope and adduct data for each compound, which can be used to help identify the compounds alongside chromatographic retention time. However, given the significant amounts of data being captured under these conditions a full mass scan doesn't typically provide the most sensitive acquisition approach unlike SIM. The SIM acquisition can focus on a particular $\mathrm{m} / \mathrm{z}$ ensuring greater signal accumulation over the duration of the chromatographic peak and greater sensitivity. This may be improved further by segmenting the chromatographic method to only record the SIM scan for the specific chromatographic peak however, this can result in false negative results by "missing" the peak for data capture by a retention time shift often encountered with complex samples.

As ibuprofen and diclofenac are normally analysed in negative ion mode, a full mass scan in both positive and negative mode were recorded. Within the chromatogram the compounds were identified by precursor $\mathrm{m} / \mathrm{z}$ as a mixture and showed good chromatographic resolution, with the exception of acetaminophen and acetaminophen-(methyl)- $\mathrm{d}_{3}$; this is unsurprising since the latter is a deuterated analogue but is capable of being distinguished due to differing precursor $m / z$. For accurate integration of the peak area and therefore quantitation of compounds, sufficient numbers of mass spectra should be recorded within the chromatographic peak. This was initially investigated by determining the data points generated when the SIM scans were grouped according to ionisation mode, however by separating out the SIM scans; having one for each compound improved the selectivity and sensitivity, with most notable improvement being observed for diclofenac. Diclofenac was analysed in both positive and negative mode to determine which achieved better sensitivity. By separating out the SIM scans it was clear that better sensitivity was seen for diclofenac in positive mode $(\mathrm{m} / \mathrm{z} 296)$ with a tenfold increase in signal intensity, therefore the SIM scan for diclofenac in negative was removed in further studies.

After segmenting the method, adjustments were made to the scan time and the inter-scan delay for the SIM scans. These two parameters combined relate to the duty cycle of the instrument, which is the overall time it takes the mass analyser to ramp the RF to DC voltage and emit the ions into the detector. The quadrupole mass analyser has potential for improved quantitation with the ability to quickly scan ions, resulting in a short duty cycle, and a greater number of mass spectra generated per second. Decreasing the inter-scan delay from the default 0.3 seconds to 0.01 seconds resulted in a small increase in the number of data points across the chromatographic peak, with the exception of ibuprofen in negative mode. Under these conditions the peak for ibuprofen disappeared, therefore an alternative inter-scan delay 
setting for the ibuprofen SIM scan was investigated, with a decrease to 0.1 seconds proving successful. Further gains in scan time were also achieved by reducing the scan time for the full mass scan from 0.8 seconds to 0.5 seconds; this resulted in an increased number of data points across the peak, however there was still less than 10 per peak. This was still considered insufficient for quantitation and therefore the mass scale recorded was reduced. As the smallest $\mathrm{m} / \mathrm{z}$ of interest is 152 and the biggest being $\mathrm{m} / \mathrm{z} 734$, a range of 125-775 Da was chosen and the scan time was further reduced to 0.4 seconds. As ibuprofen is the only compound being analysed in negative mode, and elutes at approximately 23 minutes, the time scale for which the negative full mass scan was recorded was reduced to incorporate this data and the wash section of the method to check for carryover on column. These changes finally resulted in 10-16 relevant SIM spectra to be captured for each compound for quantitation.

\subsection{1: Separation and Column Chemistry}

The mobile phases used comprised of $0.1 \%$ formic acid in water (A) and $100 \%$ acetonitrile (B), as these conditions had been used in the literature $[15,16]$ and in-house for the separation of pharmaceuticals. The initial LC method used a 31 minute linear ramp, starting at $95 \%$ A:5\% B with appropriate wash and conditioning phases. A number of different column chemistries were investigated to evaluate which provided the best retention of the range of chemistries (acidic and basic) within the suite.

\subsubsection{1: Xbridge C18 Column}

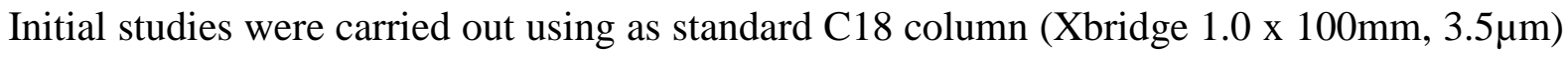
as this is the traditional separation platform for reversed-phase chromatography. The Xbridge column comprises of $\mathrm{C} 18$ chains that are bonded to the silica particle using additional bonding i.e. an ethylene-bridge hybrid particle. This is designed to increase the robustness of the column for more polar solvent conditions, and reduce any secondary interactions not captured with endcapping that may adversely affect the retention of polar compounds. Unfortunately, acetaminophen showed little retention on this column and eluted during the solvent front of the chromatographic run, resulting in an inability to accurately distinguish it from other un-retained matrix interferences. Also, ibuprofen did not appear to be retained on this column too and was not seen in negative mode using this column type. Therefore to increase retention several changes to the mobile phase composition including acidifying this acidic analyte by using $0.1 \%$ formic acid in mobile phase $\mathrm{B}$, and altering the gradient run to $99.5 \% \mathrm{~A}: 0.5 \% \mathrm{~B}$, to enhance the polarity of the starting conditions to capture acetaminophen 
were investigated. Unfortunately, neither change had a positive impact on the retention of these compounds so alternative mobile phase additives were investigated. For example, ammonium formate has been used as a mobile phase buffer for the analysis of polar pharmaceuticals [17] with the aim regulating the $\mathrm{pH}$ of the chromatographic conditions to a range of 8.2-10.2 [18], stabilising the more basic (and polar) target analytes, ensuring maximum retention on column. While these conditions resulted in improved retention of acetaminophen, the chromatography was not stable with peak retention times shifting for successive runs, therefore was not investigated further and alternative column chemistries considered.

\subsubsection{2: Xselect Charged Surface Hybrid (CSH) Column}

The Xselect $\mathrm{CSH}$ column was investigated as an alternative to the Xbridge C18 as the permanently charged bead surface of the Xselect is designed to increase the retention of basic compounds. As the dimensions of this particular column differed slightly from the Xbridge; a $2.1 \times 150 \mathrm{~mm}, 3.5 \mu \mathrm{m}$ column, the mobile phase flow rate was altered to accommodate the wider bore of this column. However the data showed that there was no improvement on the retention of acetaminophen, with elution occurring within the solvent front therefore this column was not investigated further.

\subsubsection{3: Xselect High Strength Silica (HSS) T3 Column}

The Xselect HSS T3 column is designed to improve retention of polar compounds as a result of higher silanophilicity/hydrophobicity of the $100 \%$ silica particles and was evaluated for any improvement of the retention of acetaminophen A $1.0 \times 100 \mathrm{~mm}, 3.5 \mu \mathrm{m}$ column equipped with a KrudKatcher in-line filter was investigated using the same mobile phase composition as used with the Xbridge C18 (0.1\% formic acid in water, and $100 \%$ acetonitrile). While acetaminophen showed improved retention and chromatography with these mobile phases, this was also apparent for the remaining pharmaceuticals with improved chromatography and peak shape observed, and less background signal from co-elution of interferences at retention times observed for target compounds that elute in the middle of the gradient (i.e. carbamazepine) as shown in Figure 3.11. The detection of ibuprofen was also improved using this column, with a signal consistent with this pharmaceutical observed in negative mode at low concentrations. 


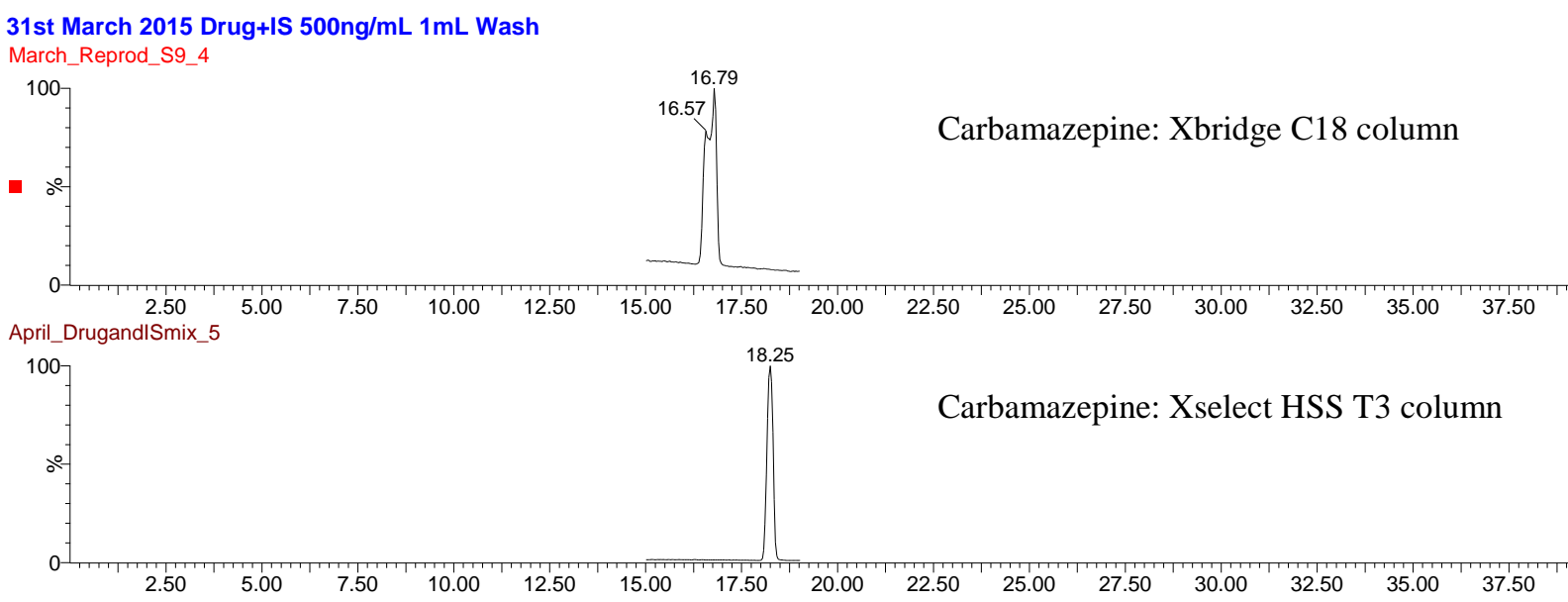

Figure 3.11: Chromatograms showing carbamazepine at a concentration of $500 \mathrm{ng} / \mathrm{mL}$ recorded on both the Xbridge and Xselect HSS T3 columns. As can be seen, the resolution and peak shape of the compound is much improved using the Xselect HSS T3 column.

\subsection{2: Chromatographic Performance}

Once separation of compounds had been achieved with the sufficient number of data points for quantitation, the LC-MS method was characterised by determining the presence of any carryover of the compounds, assessing the chromatographic repeatability and reproducibility. The initial composition of the wash solution was a 50:50\% mixture of acetonitrile and water, as used in previous in-house work. For the compounds that were un-retained, it was challenging to characterise carryover; with difficulties in discerning these analytes from the solvent front. Various wash solutions were investigated, including more aqueous mixtures, acidification of the solution, alternative solvents (i.e. methanol) as well as various wash volumes to mitigate carryover and evaluate if the wash solution influences retention, given its use as a flush solvent in the injection programme. A mixture of 25\%: $75 \%$ acetonitrile and water respectively, acidified with $0.1 \%$ formic acid and a wash volume of $1 \mathrm{~mL}$ proved to be the optimum injection wash conditions with no detectable carryover following multiple injections of a high concentration standard.

\subsubsection{1: Comparison of Column Performance}

Chromatographic performance of both the Xbridge C18 and the Xselect HSS T3 columns was evaluated by analysing repeat injections of a high concentration standard to characterise the column performance, repeatability and reproducibility to determine which column technology was most appropriate for the suite of pharmaceuticals. This was achieved by comparing the retention factor, selectivity factor, resolution and efficiency for each compound on both chromatographic columns, detailed in Tables 3.1 and 3.3. 


\subsubsection{1: Xbridge C18 Column: Chromatographic Separation Performance}

The retention factor $(\mathrm{k}-\mathrm{Eq}$ 1.2) for each of the eleven compounds detected using the Xbridge C18 column was greater than the ideal $(2<\mathrm{k}<10)$, demonstrating good retention efficiency. The column efficiency ( $\mathrm{N}-\mathrm{Eq} 1.1)$, relating to the number of theoretical plates is high for each compound, indicating the LC method is suitable for analysis. The selectivity factor ( $\alpha-\mathrm{Eq} 1.3)$ and resolution (Rs - Eq 1.4) for seven out of the eleven compounds fall below the acceptable value of $\alpha>1.1$ and $\mathrm{Rs}>1.5$ (equivalent to $99.7 \%$ resolved [19]), indicating separation and resolution of compounds is poor using this chromatographic column. As each compound within this method is detected using an individual SIM scan, evaluation of retention time reproducibility should indicate whether the poor separation and resolution would be problematic for this analysis, as if the retention time is not reproducible it could suggest interference with other compounds.

Table 3.1: Chromatographic performance for separation of pharmaceuticals and internal standards for the Xbridge C18 column. Retention times $\left(t_{R}\right)$ and figures of merit are given in order of elution. Values $\alpha$ and Rs are shown as selectivity and resolution from the successive compound (e.g. pronethalol from propranolol).

\begin{tabular}{|l|c|c|c|c|c|}
\hline Compound & $\begin{array}{c}\text { Mean } \\
\text { Retention } \\
\text { Time (RT) }\end{array}$ & $\begin{array}{c}\text { Retention } \\
\text { Factor } \\
(\mathbf{k})\end{array}$ & $\begin{array}{c}\text { Selectivity } \\
\text { Factor }(\boldsymbol{\alpha})\end{array}$ & $\begin{array}{c}\text { Efficiency } \\
(\mathbf{N})\end{array}$ & $\begin{array}{c}\text { Resolution } \\
(\mathbf{R s )}\end{array}$ \\
\hline Pronethalol & 13.32 & 8.3 & 1.2 & 40377.4 & 8.5 \\
\hline Propranolol & 15.23 & 9.7 & 1.1 & 108086.5 & 4.5 \\
\hline Diphenhydramine & 16.05 & 10.2 & 1.0 & 120087.7 & 0.3 \\
\hline Erythromycin & 16.11 & 10.3 & 1.0 & 77765.1 & 1.1 \\
\hline Citalopram & 16.36 & 10.5 & 1.0 & 106490.9 & 1.2 \\
\hline Carbamazepine & 16.87 & 10.8 & 1.0 & 11039.8 & 0.3 \\
\hline 10,11-DHC & 17.05 & 10.9 & 1.0 & 13530.7 & 1.0 \\
\hline Talopram & 17.43 & 11.2 & 1.1 & 131824.9 & 5.9 \\
\hline Fluoxetine & 18.56 & 12.0 & 1.0 & 152102.4 & 0.9 \\
\hline Loratadine & 18.72 & 12.1 & 1.2 & 146862.6 & 21.2 \\
\hline Diclofenac & 22.95 & 15.1 & & 202563.5 & \\
\hline
\end{tabular}

\subsection{1: XBridge C18Column: Chromatographic Repeatability and Reproducibility}

Chromatographic repeatability is "the closeness of agreement between independent results obtained with the same method on identical test material, under the same conditions (same operator, same apparatus, same laboratory and after short intervals of time)" [20]. This was determined by calculating the relative standard deviation (\%RSD) of the peak areas of multiple injections of the same sample on two separate days. The \%RSD of the adjusted 
retention times were calculated for each compound on both days and were statistically analysed as a measure of reproducibility using a two-tailed F-test. For the 8 compounds and 3 internal standards analysed using the Xbridge column, data showed little variation in retention times per day, with \%RSD less than $2 \%$. However, when analysed using an F-test significant difference was observed between day one and day two, demonstrated by a decrease in variation of retention time for day two to less than $1 \%$, indicating an improvement in chromatographic stability (see Table 3.2). In preparation for quantitation, the variability in peak area was also characterised to assess the repeatability of the autosampler. The results showed there was between $5-16 \%$ variation between the multiple injections, with pronethalol and erythromycin showing the greatest variation at $12.23 \%$ and $15.70 \%$ respectively. This data is shown in full in Appendix 3.2.

Table 3.2: Table shows the summary of reproducibility data obtained using the Xbridge C18 column. The relative standard deviation (\%RSD) values for each compound are shown for both data sets and the F-test value calculated showing that while the variation is significantly different over the two days, the chromatography is deemed reproducible.

\begin{tabular}{|c|c|c|c|}
\cline { 2 - 4 } \multicolumn{1}{c|}{} & \multicolumn{2}{c|}{ Adjusted Retention Time } & $\begin{array}{c}\text { Critical Value: } \\
\mathbf{F}_{(\mathbf{9 , 5})} \mathbf{6 . 6 8 1}\end{array}$ \\
\hline Compound & $\begin{array}{c}\text { \% RSD Day 1 } \\
(\mathbf{n = 1 0 )}\end{array}$ & $\begin{array}{c}\text { \%RSD Day 2 } \\
(\mathbf{n = 6 )}\end{array}$ & F-Test \\
\hline Pronethalol & 1.68 & 0.35 & 23.23 \\
\hline Propranolol & 0.93 & 0.24 & 15.35 \\
\hline Diphenhydramine & 0.82 & 0.17 & 24.33 \\
\hline Citalopram & 0.71 & 0.17 & 16.61 \\
\hline Erythromycin & 0.79 & 0.23 & 11.69 \\
\hline Carbamazepine & 0.77 & 0.19 & 16.99 \\
\hline 10,11-DHC & 0.46 & 0.13 & 11.71 \\
\hline Fluoxetine & 0.47 & 0.05 & 80.00 \\
\hline Talopram & 0.60 & 0.15 & 16.67 \\
\hline Loratadine & 0.57 & 0.15 & 14.34 \\
\hline Diclofenac & 0.32 & 0.12 & 7.20 \\
\hline
\end{tabular}

\subsubsection{2: Xselect HSS T3 Column: Chromatographic Separation Performance}

The retention factor $(\mathrm{k}-\mathrm{Eq} 1.2)$ for each compound was greater than the ideal $(2<\mathrm{k}<10)$, demonstrating good retention efficiency, with the exception of acetaminophen and the deuterated homolog, acetaminophen-(methyl)- $\mathrm{d}_{3}$, which had a $\mathrm{k}$ of 0.4 , showing that these compounds eluted close to the solvent front. Although these values are above the recommended values, the column efficiency $(\mathrm{N}-\mathrm{Eq} 1.1)$ is high, indicating the LC method is 
appropriate. The selectivity factor ( $\alpha-\mathrm{Eq} 1.3)$ and resolution ( $\mathrm{Rs}-\mathrm{Eq} 1.4)$ for the majority of the compounds was good, with $\alpha>1$ and Rs $>1.5$ (equivalent to 99.7\% resolved [19]), indicating sufficient separation of compounds. Although select pharmaceuticals show poor selectivity and resolution (e.g. acetaminophen-(methyl)- $\mathrm{d}_{3}$, erythromycin and carbamazepine), the analysis should be unaffected as each compound is detected using individual scan filters (i.e. SIM scans), providing retention time is reproducible.

Table 3.3: Chromatographic performance for separation of pharmaceuticals and internal standards for the Xselect HSS T3 column. Retention times $\left(t_{R}\right)$ and figures of merit are given in order of elution. Values $\alpha$ and Rs are shown as selectivity and resolution from the successive compound (e.g. acetaminophen-(methyl)- $\mathrm{d}_{3}$ from acetaminophen).

\begin{tabular}{|c|c|c|c|c|c|}
\hline Compound & $\begin{array}{c}\text { Mean } \\
\text { Retention } \\
\text { Time }\left(t_{\mathbf{R}}\right)\end{array}$ & $\begin{array}{l}\text { Retention } \\
\text { Factor (k) }\end{array}$ & $\begin{array}{l}\text { Selectivity } \\
\text { Factor }(\alpha)\end{array}$ & Efficiency (N) & $\begin{array}{l}\text { Resolution } \\
\text { (Rs) }\end{array}$ \\
\hline Acet- $d_{3}$ & 1.56 & 0.4 & 1.0 & 1276.0 & 0.0 \\
\hline Acetaminophen & 1.56 & 0.4 & 28.7 & 1481.2 & 63.3 \\
\hline Pronethalol & 13.93 & 11.5 & 1.1 & 58941.5 & 7.7 \\
\hline Propranolol & 15.61 & 13.0 & 1.1 & 89836.1 & 2.7 \\
\hline Erythromycin & 16.23 & 14.0 & 1.0 & 108328.6 & 1.0 \\
\hline Diphenhydramine & 16.47 & 13.7 & 1.0 & 92035.6 & 1.6 \\
\hline Citalopram & 16.80 & 13.5 & 1.1 & 64870.0 & 4.4 \\
\hline Talopram & 17.69 & 15.2 & 1.0 & 28427.5 & 1.3 \\
\hline Carbamazepine & 18.11 & 15.3 & 1.0 & 31743.1 & 0.4 \\
\hline 10,11-DHC & 18.26 & 14.8 & 1.1 & 122223.0 & 1.7 \\
\hline Fluoxetine & 18.76 & 15.8 & 1.0 & 147767.6 & 2.3 \\
\hline Loratadine & 19.25 & 16.2 & 1.3 & 122672.8 & 24.6 \\
\hline Diclofenac & 24.67 & 21.1 & 1.0 & 198512.1 & 1.6 \\
\hline Ibuprofen & 25.01 & 21.4 & & 222667.1 & \\
\hline
\end{tabular}

\subsection{1: Xselect HSS T3Column: Chromatographic Repeatability and Reproducibility}

The retention and injection repeatability and reproducibility was assessed for the pharmaceuticals retained using this column. The suite of pharmaceuticals now comprised of 10 compounds, with the inclusion of acetaminophen and ibuprofen, and an additional internal standard, acetaminophen-(methyl) $-\mathrm{d}_{3}$. Once the SIM transitions were assessed to ensure a sufficient number of data points were still achieved upon addition of three extra SIM scans and a full mass scan in negative mode, carryover, chromatographic repeatability and reproducibility could be confirmed and characterised, respectively The \%RSD for the adjusted retention times of all compounds, on both days was repeatable showing a \%RSD 
less than 5\%, over multiple injections. The method also showed improved chromatography for all compounds with time apart from acetaminophen and acetaminophen-(methyl)- $\mathrm{d}_{3}$, which increased from $2 \%$ to $5 \%$. Despite increased \%RSD for these compounds, the F-test showed that there was no significant difference between the variances, therefore the chromatography and method was deemed reproducible, and the full data set is shown in Appendix 3.3. As with the Xbridge C18 column, injection repeatability was established by calculating \%RSD of the peak areas of repeat injections of a single high concentration standard, at $400 \mathrm{ng} / \mathrm{mL}$, on a single day and compared to determine reproducibility using the F-test statistical test. The data shows there is less than $7 \%$ variability between the repeat injections on day one and less than $5 \%$ on day two. This decrease in variation of peak area suggested that an overall improvement occurred, and given the ability to detect additional compounds, the Xselect HSS T3 column was chosen and characterisation of the method for quantitation was carried out.

Table 3.4: Table shows the summary of injection repeatability data obtained using the Xselect HSS T3 column. The relative standard deviation (\%RSD) values for each compound are shown for both obtained on both days and the F-test value calculated showing that the variation shown over two separate days isn't significant.

\begin{tabular}{|c|c|c|c|c|}
\cline { 2 - 5 } \multicolumn{1}{c|}{} & \multicolumn{2}{c|}{ Peak Area } & $\mathbf{F}_{\mathbf{( 9 , 5 )}} \mathbf{6 . 6 8 1}$ & $\mathbf{F}_{(\mathbf{5 , 9})} \mathbf{4 . 4 8 4}$ \\
\hline Compound & $\begin{array}{c}\text { \%RSD Day 1 } \\
(\mathbf{n = 1 0})\end{array}$ & $\begin{array}{c}\text { \% RSD Day } \mathbf{2} \\
(\mathbf{n = 6})\end{array}$ & F-Test & F-Test \\
\hline Acet-d $_{3}$ & 6.85 & 5.03 & 1.94 & \\
\hline Acetaminophen & 1.38 & 1.16 & 1.54 & \\
\hline Pronethalol & 2.33 & 1.13 & 4.39 & \\
\hline Propranolol & 1.34 & 0.77 & 3.08 & \\
\hline Diphenhydramine & 1.80 & 1.65 & 1.15 & \\
\hline Citalopram & 1.59 & 1.85 & & 1.29 \\
\hline Erythromycin & 1.34 & 1.08 & 1.74 & \\
\hline Carbamazepine & 4.52 & 1.59 & 9.19 & \\
\hline 10,11-DHC & 1.86 & 0.55 & 11.75 & \\
\hline Fluoxetine & 1.45 & 1.01 & & 2.20 \\
\hline Talopram & 2.15 & 2.99 & & 1.87 \\
\hline Loratadine & 3.26 & 1.40 & 6.25 & \\
\hline Diclofenac & 1.38 & 0.70 & & 4.22 \\
\hline Ibuprofen & 4.87 & 2.69 & 2.79 & \\
\hline
\end{tabular}

\section{3: Quantitation using Xselect HSS T3 Column}

To characterise the ability of an analytical method for performing reliable quantitation the construction of a calibration graph comprising of a series of standards prepared over range of 
concentrations is required. For mass spectrometry methods, internal standards are employed to normalise the analyte signal and account for fluctuations of sensitivity caused by matrix interference. Therefore, internal standards should be as chemically similar to the target analyte requiring quantitation as possible and will require evaluation given structural analogues are being used here. A concentration range of 1-400 ng/mL was chosen based upon previous data, with screen samples showing this range as fit-for-purpose, and initial sensitivity tests performed by injecting serial dilutions of standard solutions during the direct infusion stage.

Table 3.5: The table shows the concentration of each calibration standard used for the characterisation of quantitation experiments using the Xselect HSS T3 column.

\begin{tabular}{|l|c|c|c|c|c|c|c|c|c|}
\hline Standard & S1 & S2 & S3 & S4 & S5 & S6 & S7 & S8 & S9 \\
\hline Concentration (ng/mL) & 1 & 5 & 10 & 25 & 50 & 100 & 200 & 300 & 400 \\
\hline
\end{tabular}

This range garnered good linearity for all pharmaceuticals studied, with $\mathrm{R}^{2}>0.99$, which demonstrates a good agreement between the relative response and concentration, supporting the use of these internal standards for the suite of pharmaceuticals.

\subsection{1: Heteroscedasticity}

Reliability of quantitative results depends upon the quality of the derived calibration graph. Regression statistics are typically used to determine the concentration of unknown samples, and the data processing should be chosen according to the characteristics of the data and measurement process. For example, different detection methods will have inherent errors in their measurement and will differ depending on the amount of signal present. This is assessed by the percentage relative error ( $\% \mathrm{RE})$, comparing the calculated concentration obtained from the regression equation with the theoretical concentration, and plotting on a graph. This process characterises the error and enables a correction to be made (i.e. weighting factor) to provide more representative data without "skewing". The correction or weighting factor is chosen according to that which gives the narrowest band of \% RE along the concentration axis [21]. Data of this type is classed as heteroscedastic, with homoscedastic data being the term used to describe equal variances across the analytical data set. For mass spectrometric data these conditions are not often met; the deviations observed for the higher concentrations in the dynamic range influence, or weight the regression line causing inaccuracy in measuring 
the lower concentrations and is typically corrected by using a weighted regression function such as $1 / \mathrm{x}$ or $1 / \mathrm{x}^{2}$.

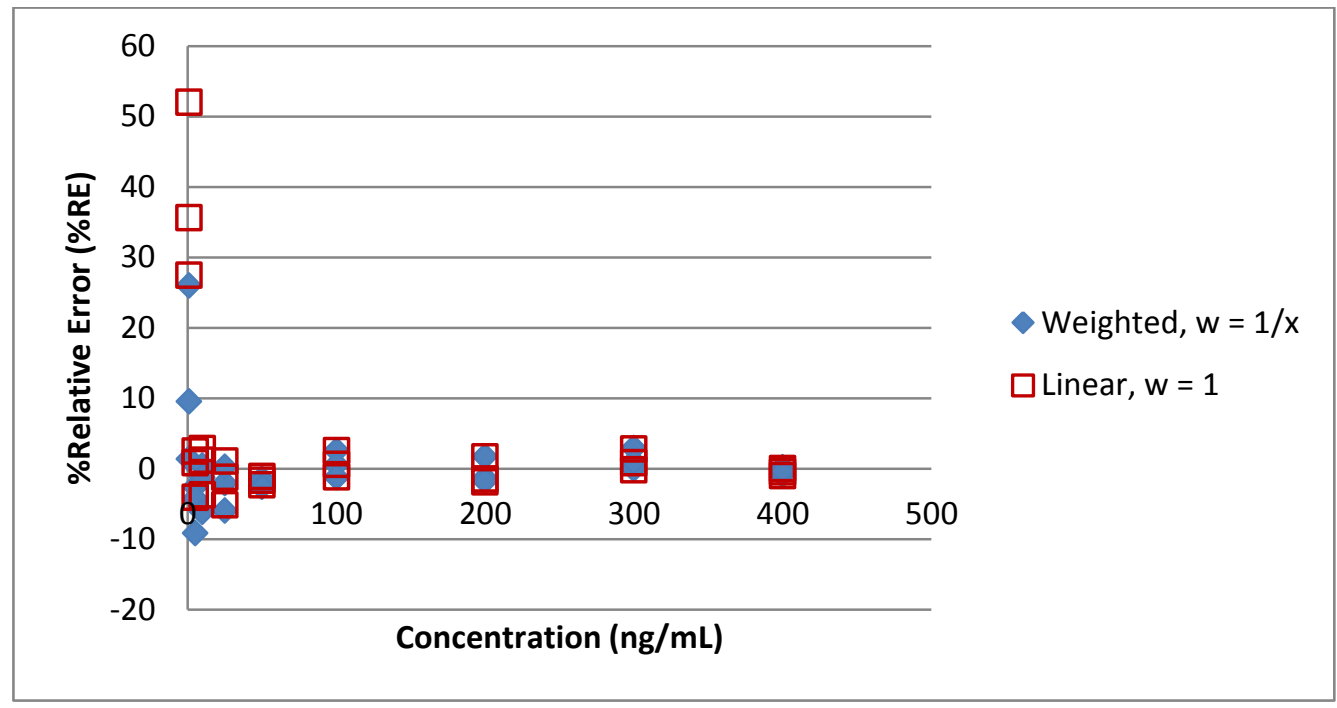

Figure 3.12: An example percentage relative error (\%RE) versus concentration graph for fluoxetine, showing the spread of data for linear regression statistics $(\square)$, and weighted 1/x regression data ( $\diamond)$. As shown, the data for $1 / \mathrm{x}$ shows less variability over the entire concentration range.

The heteroscedasticity of data obtained using the T3 column was assessed, and showed a heteroscedastic data set which improved significantly with a smaller, more equal variance for all concentrations following application of a weighted regression function of $1 / x$, clearly shown in Figure 3.12.

\subsection{2: Instrument Detection Limit (IDL)}

There are many ways to statistically determine the instrument detection limit (IDL) of a compound. For method evaluation two different methods were chosen; a statistical determination, as described by Miller and Crowther (Eq. 2.6) [22] and an empirical method using an internal standard blank, S0 (Eq. 2.7), as described in the NS30 document, A Manual on Analytical Quality Control for the Water Industry [23]. When calculated statistically, the IDL values for the compounds in the study were between $5-30 \mathrm{ng} / \mathrm{mL}$. This did not correlate with the data obtained with chromatographic peaks observed with signal-to-noise $(\mathrm{S} / \mathrm{N})$ of approximately $3: 1$ at the lower end of the concentration range $(1 \mathrm{ng} / \mathrm{mL})$ for all compounds. Using the empirical determination of NS30, values of less the $1 \mathrm{ng} / \mathrm{mL}$ were determined, with the exception of acetaminophen $(5.9 \mathrm{ng} / \mathrm{mL})$. This difference in these calculated values may be due to the heteroscedasticity of the data and will require testing. For example, Miller and 
Miller state that transposing the standard error of regression $\left(\mathrm{S}_{\mathrm{y} / \mathrm{x}}\right)$ required for the statistical determination of the IDL, for the standard deviation of the blank $\left(S_{B}\right)$ can be used for homoscedastic data to make determining the IDL more manageable [24]. However, given the noted variation in the calibration data this approximation may not be appropriate and determining IDL using a blank sample may be more representative of the IDL and these values are far more aligned with the $\mathrm{S} / \mathrm{N}$ estimates of $3: 1$, therefore the empirical data was considered a more appropriate description of method performance.

\subsection{3: Precision and Accuracy}

To assess precision and accuracy, rigorous acceptance criteria that are used within the pharmaceutical industry whereby accuracy and precision are determined by quality controls (QCs) at key concentrations required for the measured concentration were adopted. It is generally accepted that the QC samples should be "within $15 \%$ of the actual concentration except at the limit of quantitation (LOQ) where it may not exceed 20\% accuracy" [25]. The precision and accuracy of the method for quantitation was determined using QC samples at four different concentrations $(15,25,100$ and $350 \mathrm{ng} / \mathrm{mL})$ which were prepared and analysed in triplicate. Using the regression equation generated in the QuanLynx software, the samples were analysed to determine the concentration present in each sample. Both inter- and intraday precision was determined using the percentage relative standard deviation (\%RSD) of the calculated concentration, and was necessary to characterise the ability of the analytical method to reliably measure an individual compound. For the QCs chosen for this analysis, the intra-day precision was less than $10 \%$ for each compound, and inter-day precision values determined over three data sets were shown to be less than $11 \%$, indicating that this method and these chromatographic conditions are suitable for reliably measuring concentration within this given range over multiple injections and over different days (see Table 3.6).

The accuracy of each replicate was determined by calculating the percentage difference between the calculated concentration and the actual spiked concentration. The QCs showed a good degree of accuracy at all concentrations for each compound, with the results falling below $15 \%$. These results comply with the acceptance criteria used, supporting the indication that the method is capable of quantitating low concentrations of pharmaceuticals $(15 \mathrm{ng} / \mathrm{mL})$ using surrogate internal standards to an appropriate degree of accuracy. 
Table 3.6: The table shows the intra- and inter-day precision values determined for the chosen analytical method. Inter-day precision was determined over three data sets.

\begin{tabular}{|c|c|c|c|c|c|}
\hline \multirow[b]{2}{*}{ Compound } & \multirow[b]{2}{*}{$\begin{array}{c}\text { Precision } \\
(\% \text { RSD })\end{array}$} & \multicolumn{4}{|c|}{$Q C s$} \\
\hline & & $\begin{array}{c}\mathrm{QC1} \\
(15 \mathrm{ng} / \mathrm{mL})\end{array}$ & $\begin{array}{c}\mathrm{QC2} \\
(25 \mathrm{ng} / \mathrm{mL})\end{array}$ & $\begin{array}{c}\text { QC3 } \\
(100 \mathrm{ng} / \mathrm{mL})\end{array}$ & $\begin{array}{c}\text { QC4 } \\
(350 \mathrm{ng} / \mathrm{mL})\end{array}$ \\
\hline \multirow{2}{*}{ Acetaminophen } & Intra-Day & 9.48 & 3.54 & 2.75 & 4.51 \\
\hline & Inter-Day & 4.40 & 3.85 & 0.74 & 2.87 \\
\hline \multirow{2}{*}{ Propranolol } & Intra-Day & 1.75 & 1.78 & 1.51 & 1.19 \\
\hline & Inter-Day & 2.80 & 3.43 & 3.17 & 1.63 \\
\hline \multirow{2}{*}{ Diphenhydramine } & Intra-Day & 3.20 & 1.70 & 1.18 & 0.35 \\
\hline & Inter-Day & 3.51 & 4.25 & 2.58 & 3.62 \\
\hline \multirow{2}{*}{ Citalopram } & Intra-Day & 2.59 & 2.84 & 2.06 & 0.86 \\
\hline & Inter-Day & 6.26 & 3.87 & 2.61 & 2.23 \\
\hline \multirow{2}{*}{ Erythromycin } & Intra-Day & 3.48 & 6.23 & 2.88 & 2.23 \\
\hline & Inter-Day & 11.32 & 11.74 & 6.36 & 4.27 \\
\hline \multirow{2}{*}{ Carbamazepine } & Intra-Day & 1.80 & 2.45 & 1.61 & 1.00 \\
\hline & Inter-Day & 4.09 & 4.79 & 3.52 & 1.02 \\
\hline \multirow{2}{*}{ Fluoxetine } & Intra-Day & 3.28 & 4.49 & 4.50 & 1.13 \\
\hline & Inter-Day & 9.47 & 8.74 & 5.51 & 4.08 \\
\hline \multirow{2}{*}{ Loratadine } & Intra-Day & 2.15 & 2.56 & 3.02 & 2.28 \\
\hline & Inter-Day & 8.44 & 7.83 & 5.95 & 3.32 \\
\hline \multirow{2}{*}{ Diclofenac } & Intra-Day & 8.58 & 10.50 & 4.42 & 0.80 \\
\hline & Inter-Day & 6.27 & 10.58 & 6.70 & 3.92 \\
\hline
\end{tabular}

\subsection{4: Instrument Quantitation Limit (IQL)}

The analytical method was further characterised to establish the instrument quantitation limit (IQL). Like the IDL values, the IQL may be determined by two different approaches; by assessing the lowest concentration QC giving a good degree of precision and accuracy $(<20 \%)$ and statistically using the standard deviation of the blank (Eq 2.8). Empirically the IQL was determined to be $15 \mathrm{ng} / \mathrm{mL}$ however, while this provides a more robust approach it may not assess what the lowest point may be as per the statistical method. Following the statistical approach often used in environmental analysis [26] (Eq.2.8), the resulting values suggest this may be significantly lower as expected however, this approach often shows poor correlation to what occurs in practice and empirical measurements will be used as a gauge of method performance.

\section{4: Stability}

The stability of the pharmaceutical stock solutions was investigated to establish how long a stock solution can stored under set conditions before the concentration of the compound 
changes significantly. The stability was assessed by preparing a set of fresh stock solutions, from which a series of calibration standards and QCs were made (t0) and compared to calibration standards and QCs prepared from 1 month old stock solutions (t28). The percentage change of the calculated concentrations for the QCs was determined and the statistical results obtained showed that six of the compounds were stable in solution within the freezer for 1 month, with a percentage change in concentration of less than $15 \%$. Propranolol, erythromycin and loratadine showed a greater percentage change, with values exceeding 25, 17 and $20 \%$, respectively. A t-test was performed on all of the compounds to determine whether the calculated concentrations were significantly different between $\mathrm{t} 0$ and t28, with results confirming the difference for propranolol and loratadine, but also showing a significant difference for citalopram ( $\%$ change in concentration $\leq 15 \%)$, with a $t$-value that exceeded t-critical (3.75 at $98 \%$ confidence). As there are no recommendations set out by the FDA guidelines for characterising stability, and a general acceptance criteria similar to that used for accuracy and precision (i.e. $<15 \%$ considered acceptable) was deemed inappropriate by the t-test results for citalopram, fresh stock solutions would be prepared before any quantitative analysis to ensure accurate results.

\section{5: Conclusion}

Liquid chromatography-mass spectrometry is considered the gold standard for analytical methods, with reversed-phase C18 methods being the predominant conditions used. The analytical method developed in this study was tested for a suite of 10 compounds, with 1 deuterated internal standard and 3 surrogate internal standards to determine their feasibility for quantitation, and evaluated in terms of stability. Data obtained shows that alternative column chemistry, the HSS T3 column, is more suited to the wide range of pharmaceuticals chosen than a standard C18 column. With these optimised chromatographic conditions, the method has a good level of sensitivity, sufficient for the concentrations of pharmaceuticals seen in previous in-house studies (1-400 $\mathrm{ng} / \mathrm{mL})$. The method also exhibits good precision and accuracy for quantitation, with values of less than $10 \%$ and $15 \%$, respectively, within the necessary measurement range. 


\section{References}

[1] Gilpin RK, Zhou W. Studies of the thermal degradation of acetaminophen using a conventional HPLC approach and electrospray ionization - mass spectrometry. Journal of Chromatographic Science. 2004; 42: 15-20.

[2] McLafferty FW, Turecek F. Interpretation of mass spectra. $4^{\text {th }}$ Edition. USA: University Science Books; 1993.

[3] Xiu-Sheng Miao and Chris D. Metcalfe, Determination of Carbamazepine and Its Metabolites in Aqueous Samples Using Liquid Chromatography-Electrospray Tandem Mass Spectrometry, Analytical Chemistry. 2003, 75, 3731-3738

[4] Smyth FW, Leslie JC, McClean S, Hannigan B, McKenna HP, Doherty B, Joyce C, O'Kane E. W. The characterisation of selected antidepressant drugs using electrospray ionisation with ion trap mass spectrometry and with quadrupole time-of-flight mass spectrometry and their determination by high performance liquid chromatography/electrospray ionisation tandem mass spectrometry. Rapid Communications in Mass Spectrometry. 2006; 20: 1637-1642.

[5] Galmier M, Bouchon B, Medelmont J, Mercier F, Pilotaz F, Lartigue C. Identification of degradation products of diclofenac by electrospray ion trap mass spectrometry. Journal of Pharmaceutical and Biomedical Analysis. 2005; 38: 790-796.

[6] Russell P, Steinberg P, Blackburn ML, Cho D, Heather R. Rapid Quantitative and Confirmational Screening for Drugs in Race Horse Urine by ESI-LC-MS/MS and $\mathrm{MS}^{3}$. USA: Thermo Fisher Scientific; 2007. Application Note: 350.

[7] Ferrer I, Heine CE, Thurman EM. Combination of LC/TOF-MS and LC/Ion Trap MS/MS for the identification of diphenhydramine in sediment samples. Analytical Chemistry. 2004; 76 (5): 1437-1444.

[8] Crowe MC, Brodbelt JS, Goolsby BJ, Hergenrother P. Characterization of Erythromycin Analogs by Collisional Activated Dissociation and Infrared Multiphoton Dissociation in a Quadrupole Ion Trap. American Society for Mass Spectrometry. 2002; 13: 630-649.

[9] Petrović M, Gonzalez S, Barceló D. Analysis and removal of emerging contaminants in wastewater and drinking water. Trends in Analytical Chemistry. 2003; 22 (10): 685-696.

[10] Salem II, Idrees J, Al Tamimi JI. Determination of loratadine in human plasma by liquid chromatography electrospray ionization ion-trap tandem mass spectrometry. Journal of Pharmaceutical and Biomedical Analysis. 2004; 34: 141-151.

[11] Stapleton MP. Sir James Black and propranolol. The role of the basic sciences in the history of cardiovascular pharmacology. Texas Heart Institute Journal.1997; 24 (4): 336-42. 
[12] Johnson RD, Lewis RJ. Quantitation of atenolol, metoprolol, and propranolol in postmortem human fluid and tissue specimens via LC/APCI-MS. Forensic Science International. 2006; 156: 106-117.

[13] Upthagrove AL, Hackett M, Nelson WL. Fragmentation Pathways of Selectively Labeled Propranolol Using Electrospray Ionization on an Ion Trap Mass Spectrometer and Comparison with Ions Formed by Electron Impact. Rapid Communications in Mass Spectrometry. 1999; 13: 534-541.

[14] Healy D. Let Them Eat Prozac: The Unhealthy Relationship Between the Pharmaceutical Industry and Depression (Medicine, Culture, and History). USA: NYU Press; 2006.

[15] Gómez MJ, Petrović M, Fernández-Alba AR, Barceló D. Determination of pharmaceuticals of various therapeutic classes by solid-phase extraction and liquid chromatography-tandem mass spectrometry analysis in hospital effluent wastewaters. Journal of Chromatography A. 2006; 1114: 224-233.

[16] La Farré M, Ferrer I, Ginebreda A, Figueras M, Olivella L, Tirapu L, Vilanova M, Barceló D. Determination of drugs in surface water and wastewater samples by liquid chromatographymass spectrometry: methods and preliminary results including toxicity studies with Vibrio fischeri. Journal of Chromatography A. 2001; 938: 187-197.

[17] Waite M, Jenkins T, McConville P. Developing a highly sensitive for the analysis of a series of $\beta$-blockers by UPLC with fluorescence and photodiode array detection. USA: Waters Corporation; 2010.

[18] McMaster MC. LC/MS: A Practical User’s Guide. USA: John Wiley \& Sons; 2005.

[19] Willoughby R, Sheehan E, Mitrovich S. A global view of LC/MS. 1st Edition. USA: Global View Publishing; 1998.

[20] IUPAC Gold Book. Available from: http://goldbook.iupac.org/L03540.html

[21] Almeida AM, Castel-Branco MM, Falcão AC. Linear regression for calibration lines revisited: weighting schemes for bioanalytical methods. Journal of Chromatography B. 2002; 774: 215.

[22] Miller JM, Crowther JB. Analytical Chemistry in a GMP Environment: A practical guide. USA: John Wiley \& Sons, Inc.; 2000.

[23] Cheeseman RV, Wilson AL. NS30 - A Manual on Analytical Quality Control for the Water Industry. Revised by: Gardner MJ. Water Research Centre; 1989.

[24] Miller JN, Miller JC. Statistics and chemometrics for analytical chemistry. Fourth Edition. London: Prentice Hall; 2000.

[25] Food and Drug Administration, Guidance for Industry: Bioanalytical Method Validation. 2001.

[26] Ripp J. Analytical Detection Limit Guidance \& Laboratory Guide for Determining Method Detection Limits. USA: Wisconsin Department of Natural Resources Laboratory Certification Program; 1996. 


\section{Chapter 4: Liquid Chromatography-Mass Spectrometry Method Development: Biocides}

\section{1: MS Detection and Identification of Biocides for Quantitation}

Given the significantly higher signal intensity of the proposed biocides in comparison to the suspected pharmaceuticals within this initial screen, an alternative ion trap platform was used to quantify biocides and undertake a more informative screen. This would not require the more challenging sensitivity of pharmaceuticals and therefore further method development was undertaken. Analysis of "pure" samples to determine the precursor and characteristic fragment ions before online LC-MS analysis is typically performed by infusion of standard solutions. Fragmentation data was obtained previously in-house by tandem mass spectrometry (MS/MS) using collision induced dissociation (CID), whereby the molecular ion is dissociated as a result of interaction with a target neutral species (helium or nitrogen) due to the conversion of part of the translational energy of the ion to internal energy within the ion during collision [1]. To confirm the precursor ions and ensure the collision energies $(\% \mathrm{CE})$ for the generation of qualifying product ions were correct for this method, a product ion scan was performed for each compound.

\subsection{1: Benzalkonium Chlorides (BACs)}

A group of four BAC compounds were studied, BAC-C12, BAC-C14, BAC-C16 and BACC18, with a deuterated BAC-C14 used as the internal standard for the suite. These compounds are available as halide salts, typically hydrochloride and are comprised of an ammonium group attached to both a benzene head group and an aliphatic carbon chain of increasing length by an addition of a methylene group, $\left(\mathrm{CH}_{2}\right)_{2}$. The precursor ions observed are consistent with the loss of the chloride ion, forming the $[\mathrm{M}-\mathrm{Cl}]^{+}$ion. The mass decrease observed within the product ion scan for each BAC compound was consistent with a loss of $92 \mathrm{Da}$, likely to be the methylbenzene head group (see Figure 4.1), leaving the aliphatic amine chain. This is confirmed by the fragmentation observed for the IS, whereby the deuterated methylbenzene is lost (99 Da), leaving the same product ion as seen with BACC14 at $m / z 240$. 


$$
\mathrm{R}=\mathrm{C}_{\mathrm{n}} \mathrm{H}_{2 \mathrm{n}+1}, \mathrm{n}=10,12,14,16
$$

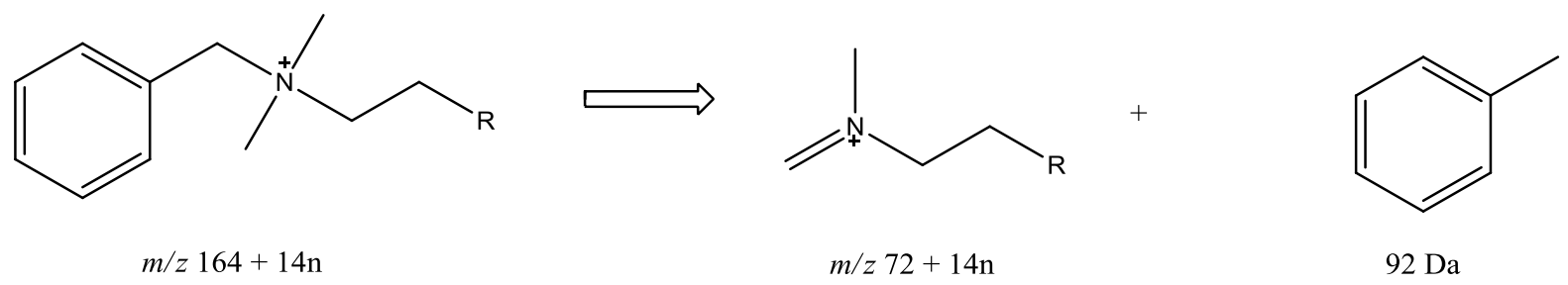

Figure 4.1: The proposed fragmentation of the BAC compounds, showing the loss of the methylbenzene ring leaving the amine chain.

\subsection{2: Didecyldimethylammonium Bromide (DDMA)}

Similarly to the BAC compounds, DDMA is supplied as a hydrogen bromide salt, and is comprised of two aliphatic carbon chains either side of a nitrogen atom, with the molecular formula $\mathrm{C}_{22} \mathrm{H}_{48} \mathrm{~N} . \mathrm{HBr}$ and monoisotopic weight of $406 \mathrm{Da}$. The loss of $\mathrm{Br}$ gives rise to a precursor ion at $m / z, 326$, consistent with $[\mathrm{M}-\mathrm{Br}]^{+}$. The product ion observed at $\mathrm{m} / \mathrm{z} 186$ (see Appendix 4.1 for spectrum), corresponds to the loss of one of the carbon chains $\left(\mathrm{C}_{10} \mathrm{H}_{20}\right)$, leaving the positively charged amine chain.

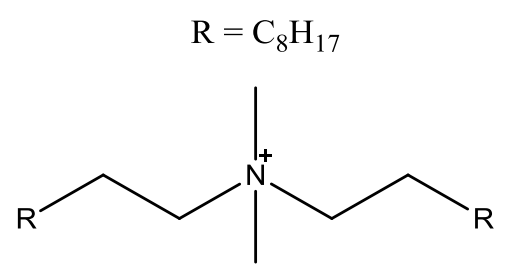

$m / z 326$

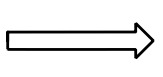

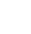

$$
m / z 186
$$<smiles>[R]C=C</smiles>

$140 \mathrm{Da}$

Figure 4.2: The proposed fragmentation of DDMA, showing the loss of one of the carbon chains leaving the amine chain.

\subsection{3: Hexadecyltrimethylammonium Chloride (HDTMA)}

The standard reference material is available as a hydrochloride salt, with a molecular formula of $\mathrm{C}_{19} \mathrm{H}_{42} \mathrm{~N} . \mathrm{HBr}$ and monoisotopic mass of $319 \mathrm{Da}$. The loss of the $\mathrm{Cl}$ gives a precursor ion at $\mathrm{m} / \mathrm{z}$ 284. Unlike the other biocides, HDTMA does not produce any stable product ions, likely a result of the structure which is an amine head group bonded to an aliphatic carbon chain $\left(\mathrm{C}_{16} \mathrm{H}_{33}\right)$; the structure of the product ions of the BACs and DDMA. 


\section{2: Semi-Quantitative Method Development for Biocides}

To develop this dual method the original LC method detailed in Chapter 3 was expanded to include the biocides and a standard mixture containing these and the pharmaceuticals were used. The SIM scans used for the pharmaceuticals within the original ZQ4000 method were replaced with product ion scans, with the aim of supporting positive identification of compounds within complex samples and distinguishing any near co-eluting species of the same precursor $\mathrm{m} / \mathrm{z}$. Pleasingly, the fragmentation patterns observed with the ion trap were the same as those seen by in-source CID on the ZQ4000, with the exception of erythromycin, where the product ion seen was at $m / z 576$, rather than $m / z 558$, corresponding with the loss of the cladinose sugar [2]. This is important to establish to ensure the data can be translated between the two platforms. Given the pharmaceuticals eluted within the first 24 minutes of the chromatographic gradient and the qualitative purpose of their analysis, the method was developed without the need to obtain a relatively high number of data points across the chromatographic, however the segmentation of the mass spectrometric method was devised to ensure a minimum of 5 data points were recorded for the targeted product ion scans. As in the initial screen the biocides were observed to elute at $82-100 \%$ acetonitrile (mobile phase B) after the pharmaceuticals, between 24 and 31 minutes. Given this chromatographic separation, this section of the method was dedicated to the quantitation of the biocides with data acquisition by selected reaction monitoring (SRM), with the exception of HDTMA; this required a SIM scan due to a lack of suitable fragmentation ions (see Table 4.1). A slightly bigger mass range of $\mathrm{m} / \mathrm{z}, 100-800$ was chosen for this method compared with the ZQ4000 method. This was achievable, without detriment to the data points across the peak due to the omission of the negative ionisation mode scans, and was used to ensure detection of all of the target precursor and product ion $\mathrm{m} / \mathrm{z}$ for qualitative and quantitative analysis. A minimum of 10 data points across the chromatographic peak was required for the accurate quantitation of the QACs, and was achieved by altering the number of microscans for both the full mass scan and the SRM/SIM scans. A microscan is one mass analysis (i.e ion injection, storage/scan-out of ions) followed by ion detection, and the time required to capture all the microscans in the mass scan refers to the duty cycle. By using a low number of averaged microscans, the duty cycle is reduced, resulting in an increase in mass scans across the chromatographic peak. When the method was tested using 1 microscan for the full mass scan and 1 for the SRM/SIM scan, 10 data points were recorded across the chromatographic peak for each QAC. 
Table 4.1: Summary of the mass spectrometer method parameters for the quantitative method for biocide analysis including the observed retention times, scan type, precursor and product ions and the associated collision energy used for each biocide.

\begin{tabular}{|l|c|c|c|c|c|}
\hline Compound & $\begin{array}{c}\text { Retention } \\
\text { Time } \\
\text { (minutes) }\end{array}$ & $\begin{array}{c}\text { Scan } \\
\text { Type }\end{array}$ & $\begin{array}{c}\text { Precursor } \\
\text { Ion }(\boldsymbol{m} / \mathbf{z})\end{array}$ & $\begin{array}{c}\text { Product } \\
\text { Ion }(\boldsymbol{m} / \mathbf{z})\end{array}$ & $\begin{array}{c}\text { Collision } \\
\text { Energy } \\
(\boldsymbol{\%})\end{array}$ \\
\hline BAC-C12 & 24.2 & SRM & 304 & 212 & 40 \\
\hline BAC-C14 & 26.4 & SRM & 332 & 240 & 42 \\
\hline BAC-C16 & 28.7 & SRM & 360 & 268 & 44 \\
\hline BAC-C18 & 30.9 & SRM & 388 & 296 & 48 \\
\hline DDMA & 27.6 & SRM & 326 & 186 & 48 \\
\hline HDTMA & 26.3 & SIM & 284 & n/a & n/a \\
\hline BAC-C14-d & 26.3 & SRM & 339 & 240 & 40 \\
\hline
\end{tabular}

\section{3: Chromatographic Performance}

Similar to the ZQ4000 method, this combined method was tested for selectivity and carryover by assessing whether there was any observed signal attributable to the compounds at high concentrations after multiple injections. This method used the same wash solution as the ZQ4000 analysis (i.e. $1 \mathrm{~mL}$ of $0.1 \%$ formic acid in a mixture of $75 \%: 25 \%$ water and acetonitrile, respectively), which proved sufficient despite the biocides typically eluting at a higher organic gradient, as no carryover was observed. The retention factor ( $\mathrm{k}-\mathrm{Eq} 1.2)$ for each of the seven compounds was greater than the ideal $(2<\mathrm{k}<10)$, demonstrating good retention efficiency. The column efficiency ( $\mathrm{N}-\mathrm{Eq}$ 1.1) calculated was also high for each compound, indicating the LC method is suitable for analysis of these compounds. The selectivity factor ( $\alpha-\mathrm{Eq} 1.3)$ and resolution ( $\mathrm{Rs}-\mathrm{Eq} 1.4)$ for the majority of the compounds fall below the acceptable value of $\alpha>1.1$ and two compounds, HDTMA and BAC-C14-d7 fall below the acceptable value of $\mathrm{Rs}>1.5$ (equivalent to $99.7 \%$ resolved [3]), indicating separation and resolution of compounds is poor using this chromatographic column. However, as the compounds will be detected using individual SRM/SIM scans, the analysis should not be affected by the poor separation and resolution provided there is no matrix suppression and the retention time is reproducible. 
Table 4.2: Chromatographic performance for separation of biocides and internal standard for the Xselect HSS T3 column. Retention times $\left(t_{R}\right)$ and figures of merit are given in order of elution. Values $\alpha$ and Rs are shown as selectivity and resolution from the successive compound (e.g. BAC-C12 from HDTMA).

\begin{tabular}{|l|c|c|c|c|c|}
\hline \multicolumn{1}{|c|}{ Compound } & $\begin{array}{c}\text { Mean Retention } \\
\text { Time }\left(\mathbf{t}_{\mathbf{R}}\right)\end{array}$ & $\begin{array}{c}\text { Retention } \\
\text { Factor }(\mathbf{k})\end{array}$ & $\begin{array}{c}\text { Selectivity } \\
\text { Factor }(\boldsymbol{\alpha})\end{array}$ & $\begin{array}{c}\text { Efficiency } \\
(\mathbf{N})\end{array}$ & $\begin{array}{c}\text { Resolution } \\
(\mathbf{R s})\end{array}$ \\
\hline BAC-C12 & 24.20 & 21.2 & 1.1 & 249567.0 & 9.9 \\
\hline HDTMA & 26.31 & 23.2 & 1.0 & 204257.6 & 0.3 \\
\hline BAC-C14-d7 & 26.38 & 23.2 & 1.0 & 191212.2 & 0.1 \\
\hline BAC-C14 & 26.42 & 23.3 & 1.0 & 142017.5 & 4.9 \\
\hline DDMA & 27.61 & 24.4 & 1.0 & 279045.5 & 4.8 \\
\hline BAC-C16 & 28.71 & 25.4 & 1.1 & 202966.3 & 8.8 \\
\hline BAC-C18 & 30.97 & 27.5 & & 226947.4 & \\
\hline
\end{tabular}

\subsection{1: Chromatographic Repeatability and Reproducibility}

As with the quantitative pharmaceutical method described in Chapter 3, the chromatographic repeatability and reproducibility was determined for the QAC compounds through multiple sample injections $\left(\mathrm{n}_{1}=10, \mathrm{n}_{2}=7\right)$. The data showed repeatable, stable chromatography over prolonged use, with \%RSDs $<0.3 \%$ for all compounds on both days of analysis. While fluctuations in \%RSD were observed between day one and day two, the F-test showed that there was no significant difference between the variances, indicating the chromatographic method was reproducible and stable between different days of analysis (see Table 4.2).

Injection repeatability was also determined to understand the error between multiple sample injections for these compounds on a single day; the \%RSD of the peak areas showed there was $<12 \%$ variability, indicating little fluctuation of the ionised signal under these chromatographic conditions.

Table 4.3: Table shows the summary of reproducibility data for the biocide compounds. The relative standard deviation (\%RSD) values for each compound are shown for both data sets and the F-test value calculated showing that the variation is not significantly different over the two days.

\begin{tabular}{|l|c|c|c|c|}
\cline { 2 - 5 } \multicolumn{1}{c|}{} & \multicolumn{2}{c|}{ Adjusted Retention Time } & $\begin{array}{c}\mathbf{F}_{(\mathbf{9 , 6})} \\
\mathbf{5 . 5 2 3}\end{array}$ & $\begin{array}{c}\mathbf{F}_{(\mathbf{6 , 9})} \\
\mathbf{4 . 3 2 0}\end{array}$ \\
\hline m/z & $\begin{array}{c}\text { \%CV Day 1 } \\
\mathbf{n = 1 0}\end{array}$ & $\begin{array}{c}\text { \%CV Day 2 } \\
\mathbf{n = 7}\end{array}$ & F-Test & F-Test \\
\hline BAC-C12 & 0.23 & 0.24 & & 1.13 \\
\hline BAC-C14 & 0.13 & 0.19 & & 1.99 \\
\hline BAC-C16 & 0.17 & 0.23 & & 1.81 \\
\hline BAC-C18 & 0.18 & 0.16 & 1.23 & \\
\hline DDMA & 0.19 & 0.19 & & 1.00 \\
\hline HDTMA & 0.16 & 0.17 & & 1.11 \\
\hline BAC-C14-d & 0.21 & 0.21 & 1.01 & \\
\hline
\end{tabular}




\section{4: Quantitation of Biocides}

A calibration experiment was performed to characterise the ability of the analytical method for reliable quantitation. A series of standards prepared to a range of different concentrations were used to construct a calibration graph for each compound. A concentration range of 2-80 $\mathrm{ng} / \mathrm{mL}$ was chosen based upon sensitivity tests performed by injecting serial dilutions of the standard solutions. A single internal standard, a deuterated analogue of BAC-C14 (BACC14- $\mathrm{d}_{7}$ ) was used for calibration at a concentration of $20 \mathrm{ng} / \mathrm{mL}$.

Table 4.4: The table shows the concentration of each calibration standard used for the characterisation of quantitation experiments for the biocides.

\begin{tabular}{|l|c|c|c|c|c|c|c|c|}
\hline Standard & S1 & S2 & S3 & S4 & S5 & S6 & S7 & S8 \\
\hline Concentration (ng/mL) & 2 & 6 & 10 & 20 & 30 & 50 & 70 & 80 \\
\hline
\end{tabular}

Following application of regression statistics this range showed sufficient linearity for all biocides, with $\mathrm{R}^{2}>0.97$, with the exception of BAC-C18, which had an $\mathrm{R}^{2}=0.96$ due to a decrease in peak area for one of the replicate injections of the highest calibration standard; when omitted, the $\mathrm{R}^{2}$ increased to 0.98 . These values support the use of the chosen internal standard for the suite of biocides. A series of QC samples, at four concentrations within the calibration range (i.e. $8,20,60$ and $80 \mathrm{ng} / \mathrm{mL}$ ), were used to evaluate the performance of method.

\subsection{1: Heteroscedasticity}

A test of heteroscedasticity was performed to determine the most appropriate weighting function for this type of analytical data. Comparison of percentage relative error calculated for both equal and $1 / \mathrm{x}$ weighted regression functions showed that while the $1 / \mathrm{x}$ weighting was more suited BAC-C12 and HDTMA, equal weighted linear regression was more suited to the rest of the suite. 
Table 4.5: Summary of the $\%$ relative error calculated to determine heteroscedasticity. Equal weighted linear and $1 / x$ weighted regression functions were compared, with linear showing less variation for the majority of the biocides.

\begin{tabular}{|l|c|c|}
\hline \multirow{2}{*}{ Biocide } & \multicolumn{2}{|c|}{ \% Relative Error } \\
\cline { 2 - 3 } & Linear & 1/x Weighting \\
\hline BAC-C12 & -4967.10 & 387.38 \\
\hline BAC-C14 & -4231.48 & -40293.33 \\
\hline BAC-C16 & -3212.08 & -5171.27 \\
\hline BAC-C18 & -2951.02 & -5144.40 \\
\hline DDMA & -4456.42 & 5898.27 \\
\hline HDTMA & -4798.25 & 1554.78 \\
\hline
\end{tabular}

As the heteroscedasticity test was inconclusive, a comparison was made between the calibration data obtained using both linear and $1 / x$ weighted regression, taking into consideration the linearity, precision and accuracy calculated for each compound. These results showed that the linearity was comparable between the two regression functions, however, while the overall accuracy for each replicate QC was slightly better with the 1/x regression, the precision values showed improvement with the linear weighted regression, therefore the linear regression function was chosen for further statistical analysis of the calibration data (summary found in Appendix 4.2).

\subsection{2: Instrument Detection Limit (IDL)}

As with the pharmaceuticals, the IDL for each biocide was determined empirically using an internal standard blank, S0, as described in the NS30 document [4], as it was considered a more representative description of the method performance. When calculated statistically, the IDL values for the compounds in the study were between $11-20 \mathrm{ng} / \mathrm{mL}$. This did not correlate with the data obtained, with chromatographic peaks observed with signal-to-noise $(\mathrm{S} / \mathrm{N})$ of approximately $3: 1$ at the lower end of the concentration range for all compounds. Using the empirical determination of NS30, values of less the $2 \mathrm{ng} / \mathrm{mL}$ were determined.

\subsection{3: Precision and Accuracy}

The precision of each biocide was assessed by establishing the \%RSD of the calculated concentrations for the five replicate QCs at each concentration. As with the pharmaceutical quantitative method, the acceptance guideline criteria used to assess precision was set at $15 \%$ RSD of the actual concentration, except at the lowest concentration where this was less than 
$20 \%$ RSD [5]. The precision and accuracy of the method for quantitation was determined using five replicate QC samples at four different concentrations $(8,20,16$ and $80 \mathrm{ng} / \mathrm{mL})$, with the intra-day percentage precision for each biocide equal to or less than $24 \%$. These values are higher than those observed for the pharmaceutical method but this could be due to the poorer regression value from the alternative mass analyser employed. The larger percentage values (highlighted in blue in Table 4.6) were analysed using the Grubbs' Test to determine whether they could be statistically omitted as outlying results. Two values were classed as outliers, the third replicate of QC2 $(20 \mathrm{ng} / \mathrm{mL})$ for BAC-C16 and the fifth replicate of $\mathrm{QC} 1(8 \mathrm{ng} / \mathrm{mL})$ for BAC-C18, therefore these replicates were omitted and the corresponding accuracy and precision value decreased to $22.18 \%$ and $7.23 \%$, respectively. The omission of the third replicate of QC2 did negatively affect the accuracy value, increasing the mean percentage value from -3.67 to -13.09 , but was still within the acceptance criteria. Inter-day precision was determined using three, independent data sets and percentage values were found to be less than $20 \%$ across the four concentrations (see Appendix 4.4). Although the mean precision for QC2 for the majority of the compounds was greater than $15 \%$, NS30 states that total error of $0.2 \mathrm{x}$ (where $\mathrm{x}$ denotes the actual concentration in the sample) is acceptable [6], and the QCs above and below in terms of concentration are within the acceptance criteria used to assess the pharmaceuticals, highlighting that values may be quantified in this range.

The accuracy of each replicate was determined by calculating the percentage difference between the calculated concentration and the actual spiked concentration. The QCs showed a good degree of accuracy at all concentrations for each compound, with the exception of HDTMA at QC1, which exceeds the acceptance criteria due to a high replicate injection, however the successive QC results fall below 15\%. These results comply with the acceptance criteria used, supporting the indication that the method is capable of quantitating biocides within the range of $8-80 \mathrm{ng} / \mathrm{mL}$ ) using a single surrogate internal standard to an appropriate degree of accuracy. 
Table 4.6: Summary of the linear weighted quantitative data including linearity $\left(\mathrm{R}^{2}\right)$, instrument detection limit $(\mathrm{IDL} \pm \mathrm{SD})$, mean percentage accuracy and precision of quality control sample (QC) concentration for each biocide. The values in red were subjected to the Grubbs' test and were found to be outliers. The amended accuracy and precision values for QC1 are 22.18 and 14.11, and for QC2 -13.09 and 7.23, respectively.

\begin{tabular}{|c|c|c|c|c|c|c|c|c|c|c|}
\hline \multirow{3}{*}{ Biocide } & \multirow{3}{*}{$\begin{array}{c}\text { Linearity } \\
\left(\mathbf{R}^{2}\right)\end{array}$} & \multirow{3}{*}{$\begin{array}{c}\text { IDL } \\
(\mathrm{ng} / \mathrm{mL})\end{array}$} & \multicolumn{8}{|c|}{ QCs } \\
\hline & & & \multicolumn{4}{|c|}{ Mean Accuracy (\%) } & \multicolumn{4}{|c|}{ Mean Precision (\%) } \\
\hline & & & QC1 & QC2 & QC3 & QC4 & QC1 & QC2 & QC3 & QC4 \\
\hline BAC-C12 & 843 & $0.30 \pm 0.10$ & 3.74 & -7.48 & -11.11 & -4.63 & 13.90 & 16.31 & 17.62 & 9.47 \\
\hline BAC-C14 & 0.9854 & $0.27 \pm 0.09$ & 8.85 & -1.97 & 1.12 & -4.64 & 9.76 & 15.96 & 20.09 & 13.27 \\
\hline $\mathrm{BAC}-\mathrm{C} 16$ & 0.9694 & $0.19 \pm 0.06$ & 17.99 & -12.42 & -3.67 & -9.86 & 17.25 & 22.60 & 13.71 & 5.09 \\
\hline BAC-C18 & 0.9604 & $0.76 \pm 0.25$ & 29.04 & -13.99 & -0.48 & -6.86 & 16.58 & 19.12 & 15.05 & 1.43 \\
\hline DDMA & 0.9794 & $0.99 \pm 0.33$ & 18.47 & -6.68 & -2.50 & -8.23 & 8.12 & 24.75 & 10.33 & 10.76 \\
\hline HDTMA & 0.9729 & $1.79 \pm 0.60$ & 26.67 & -5.43 & 0.50 & -4.29 & 7.30 & 13.60 & 10.95 & 7.23 \\
\hline
\end{tabular}

\section{5: Conclusion}

As part of the analytical method development, a second method for the combined qualitative and quantitative analysis of pharmaceuticals and biocides, respectively was investigated. A suite of six biocides and one deuterated internal standard was tested to determine their feasibility for quantitation. The calibration performance of this method was limited, with the $\mathrm{R}^{2}$ values determined to be $>0.97$. Typical $\mathrm{R}^{2}$ values desirable for quantitation are $>0.99$, therefore this method would not meet the strict criteria of a full method validation. However, for the purpose of this "proof-of-concept" study, the method was deemed suitable. While the precision and accuracy was varied at $24 \%$ and $25 \% \mathrm{RSD}$, respectively, exceed the acceptance criteria used for the pharmaceutical method, possibly attributable to the alternative mass analyser used, the method was deemed fit for purpose based upon the NS30 guidelines for environmental analysis [6] and the data obtained using the HSS T3 column shows that the method has a good level of sensitivity for the suite of biocides, sufficient for the concentrations seen in previous in-house studies $(2-80 \mathrm{ng} / \mathrm{mL})$. 


\section{References}

[1] IUPAC Gold Book. Available from: https://goldbook.iupac.org/html/C/C01167.html

[2] Crowe MC, Brodbelt JS. Characterization of Erythromycin Analogs by Collisional Activated Dissociation and Infrared Multiphoton Dissociation in a Quadrupole Ion Trap. American Society for Mass Spectrometry. 2002; 13: 630-649.

[3] Willoughby R, Sheehan E, Mitrovich S. A global view of LC/MS. 1st Edition. USA: Global View Publishing; 1998.

[4] Cheeseman RV, Wilson AL. NS30 - A Manual on Analytical Quality Control for the Water Industry. Revised by: Gardner MJ. Water Research Centre; 1989.

[5] Food and Drug Administration, Guidance for Industry: Bioanalytical Method Validation. 2001.

[6] Cheeseman RV, Wilson AL. NS30 - A Manual on Analytical Quality Control for the Water Industry. Revised by: Gardner MJ. Water Research Centre; 1989. 


\section{Chapter 5: Sample Preparation Method Development}

Pharmaceuticals are suspected persistent organic pollutants and the detection of these compounds within matrices such as wastewater fractions is problematic due to the sample complexity and the trace amounts of pharmaceutical to be measured. There are various methods adopted by industry to analyse complex environmental matrices that include soil and wastewater effluent, such as the United States Environmental Protection Agency (EPA) Method 1694: Pharmaceuticals and Personal Care Products in Water, Soil, Sediment, and Biosolids by HPLC/MS/MS, published in December 2007 [1]. This is an extensive, multistep analysis of persistent organic pollutants within aqueous and solid matrices, involving solid-liquid extraction, evaporation and $\mathrm{pH}$ adjustment before separation into acidic and basic SPE fractions for further clean-up before LC-MS/MS analysis. The multitude of procedures required to carry out this method are time consuming and laborious, increasing operational costs and making this unsuitable for high-throughput analysis. Also, the differing performance of this protocol has led to other regulatory agencies and stakeholders in the wastewater sector to develop their own protocols. These can also be lengthy, involving solvent-based extraction and dilution, coupled with on-line SPE before analysis by LCMS/MS [2], with separate protocols required for the other sample types (i.e. effluent and biota). As a result, methods to extract and quantitate these compounds from this type of sample matrix are necessary and of use to the Chemical Investigation Programme. Pilot inhouse data generated from a feasibility study using a standardised QuEChERS method (EN Method [3]) has shown potential in extracting pharmaceuticals [4] and biocides present from locally sourced, treated sludgecake. This feasibility study was limited in scope, without characterisation and optimisation of the protocol in understanding the efficacy and breadth of the extraction. Given the many advantages of the QuEChERS method this approach was investigated further with the aim of resolving some of the issues associated with the recognised (current) sample preparation methods.

\section{1: QuEChERS Extraction Methods}

This is a two-step sample preparation method, initially developed for the extraction of pesticides in fruit and vegetables. As the QuEChERS method has been designed to be a rough-and-ready approach to sample clean-up, it may be easily modified for high-throughput 
analyses, targeting the removal of specific classes of matrix interference to enable the detection of a broad range of sample constituents.

\subsection{1: Standardised Methods}

As discussed in Chapter 1, there are three standardised methods developed by Michelangelo Anastassiades and Steven Lehotay; the original, unbuffered method [5], the European Standards (EN) method (citrate buffers) [3] and the AOAC International method (acetate buffers) [6]. All three methods were initially investigated to determine the effect of buffers on the broad range of chemistries being studied in this project and if a product could be purchased "as is" without the need for further development. This initial testing was primarily concerned with the repeatable recovery of the pharmaceuticals given they represented the full range of polar organic pollutants (acid and base). The extractions would be evaluated under "ideal" conditions, using water, to enable the best chance of detecting any changes of recovery and whether the extraction itself contributed to any matrix interferences.

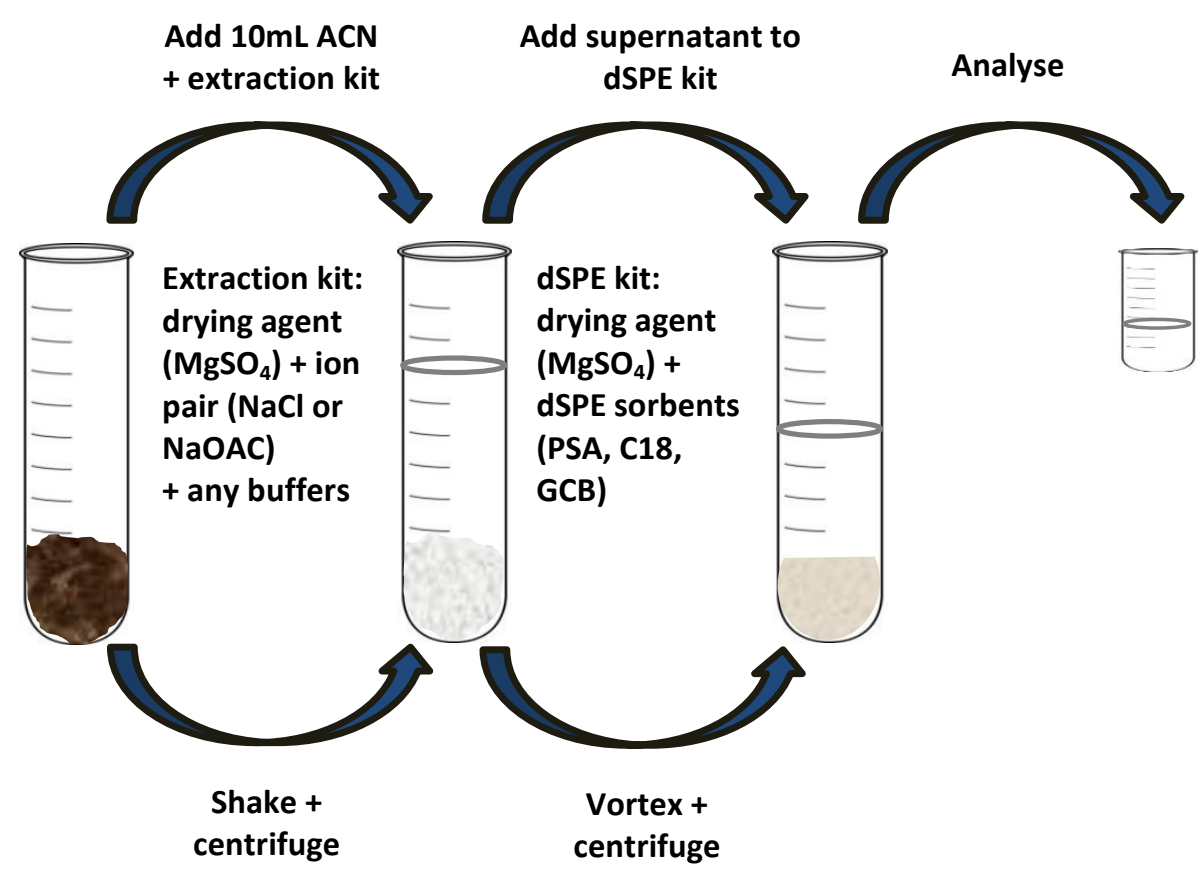

Figure 5.1: Schematic of the QuEChERS extraction procedure, including all components used in the standardised methods.

\subsubsection{1: Unbuffered QuEChERS Extractions}

The unbuffered experiment was conducted using extraction kits weighed following the specifications detailed in the original QuEChERS method [5], i.e. $4 \mathrm{~g}$ of magnesium sulphate 
and $1 \mathrm{~g}$ of sodium chloride and also an unbuffered AOAC method, i.e. $6 \mathrm{~g}$ of magnesium sulphate only. Using the same volumes as previous in-house studies, an initial spike volume of $500 \mu \mathrm{L}$ was evaporated to dryness before the addition of $4 \mathrm{~mL}$ of water, to ensure the amount of acetonitrile in the spike did not alter the chemistry of the water sample matrix, and affecting the efficiency of the extraction. The determination of matrix effects and recovery for each compound followed the method detailed in Matuszewski et al. [7], comparing the peak area of the target compound in a "spike before" and "spike after" extraction sample to provide the recovery measurement, and the peak area of the "spike after" extraction compared to a standard to determine matrix effects. The results of the original unbuffered experiment showed that while the recovery of each target compound was good (typically 30$75 \%$ ), for the majority of compounds eluting in the middle of the chromatographic run (14 20 minutes), peak broadening was observed (see Figure 5.2), which exaggerated the recovery measurement. The matrix effects measurements calculated were varied; the compounds affected by peak broadening showed matrix effects of approximately $250-310 \%$ and significant signal enhancement, while the remaining compounds were around 97-160\%.
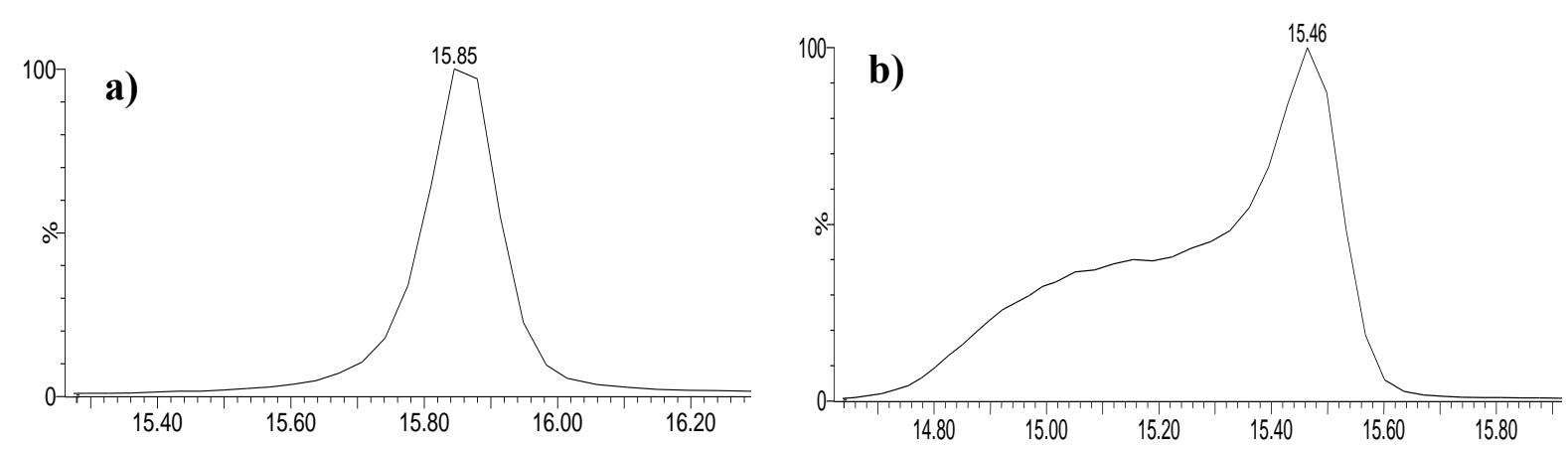

Figure 5.2: Chromatograms of propranolol to show the effect of $\mathrm{NaCl}$ on peak broadening; a) propranolol standard b) propranolol after extraction using the standardised EN QuEChERS method.

The results of the unbuffered AOAC experiment showed no alteration to the chromatographic peak shape, therefore the negative effect on chromatography observed was deduced to be due to the presence of $\mathrm{NaCl}$ in the $\mathrm{EN}$ extraction kit. The results of this experiment showed the matrix effects measurements were good, ranging between 95-140\%, indicating slight ionisation enhancement for some compounds, however, precision was poor with \%RSD of $20-36 \%(n=3)$, indicating that the measurements are not reproducible under these conditions. The recovery for the target compounds was also poor, with percentages between $0-40 \%$ $(\% \mathrm{RSD} \leq 20)$, which could be due to the lack of buffer in the extraction kit. 


\subsubsection{2: Buffered QuEChERS Extractions}

To determine whether the poor recovery of target pharmaceuticals and the change in chromatographic peak shape was a due to the lack of buffer, the experiment was repeated using commercially available buffered extraction kits. The results showed that the buffers in both methods (sodium acetate in AOAC method and sodium citrate in the EN method) had little improvement on the results of the unbuffered experiment; the chromatographic peak shape still showed peak broadening using the EN method, and the recovery was still poor using the AOAC method. Given these results it was assessed that the buffer was not the overriding factor in the limited performance and chromatographic aberrations observed.

\subsection{2: Modifications}

To investigate if the efficiency and performance of the protocol could be improved, specifically the chromatography, matrix effects and recovery measurements, a number of modifications to the unbuffered QuEChERS method were tested. Firstly, the initial evaporation step was removed to reduce the time taken to carry out the protocol for more high-throughput applications. However, to do this a smaller, more concentrated spike volume was required to minimise the influence of the "spike solvent"; reducing the $500 \mu \mathrm{L}$ spike to $40 \mu \mathrm{L}$, to give the same concentration of $100 \mathrm{ng} / \mathrm{mL}$ would mean the amount of acetonitrile in the spike would be negligible, and was suitable for direct addition into the water sample matrix for subsequent modification experiments.

\subsubsection{1: Evaluation of Initial Extraction Kit}

From the results of the initial unbuffered experiment, it was shown that extractions involving $1 \mathrm{~g}$ of sodium chloride had a negative effect on the chromatographic peak shape of some of the pharmaceuticals. Therefore, the impact of salt was investigated by comparing extractions using half the original amount of salt $(0.5 \mathrm{~g})$ and no salt, leaving just $4 \mathrm{~g}$ of magnesium sulphate within the initial extraction tube. Interestingly, the results without salt improved; the chromatographic peak shape was observed as a more Gaussian peak shape indicating a good degree of retention on column. However, the matrix effects and recovery of the target pharmaceuticals observed were poor $(\% \mathrm{ME} \leq 66 \%$, indicating ionisation suppression, and $\%$ REC $\approx 40 \%$ ) therefore the method needed further adjustment. In an attempt to improve recovery without compromising the chromatography, alternative ion pairs to sodium chloride were investigated. 
There have been a number of studies within the literature that describe modifications to the ion pair $[8,9]$ including the addition of disodium-EDTA. This was investigated to help reduce potential loss from the initial extraction step. However, this led to resulting extracts that required extended time to evaporate to dryness (several hours for $6 \mathrm{~mL}$ extract) as part of the solvent exchange step for LC-MS analysis. The results of this experiment also showed a formation of an immiscible layer after centrifugation, potentially caused by the displacement of the sodium ions with magnesium from the $\mathrm{MgSO}_{4}$, forming magnesium-EDTA. To assess this theory sodium-EDTA was used in conjunction with sodium sulphate $\left(\mathrm{Na}_{2} \mathrm{SO}_{4}\right)$ as an alternative to $\mathrm{MgSO}_{4}$ as described in Chuang et al. [8]. However $\mathrm{Na}_{2} \mathrm{SO}_{4}$ appeared to have little impact as a drying agent, failing to remove the water from the sample tube after centrifugation, and was therefore not investigated further.

\subsubsection{1: Mix and Match Approach to Extraction}

One of the key aspects of creating a modified QuEChERS method is to ensure that it could be a cost effect sample preparation method, easily transferrable to a high-throughput industrial laboratory. After identifying other potential ion pairs, such as magnesium chloride or magnesium-EDTA, it was decided that complicating the extraction kit would potential make the method unattractive to future users, therefore keeping to the chemical ingredients of the kit may offer the greatest potential in meeting this aim. From the initial experiments involving the AOAC and EN methods, results showed that of the initial extraction kits:

1) $\mathrm{AOAC}$ method ( $6 \mathrm{~g} \mathrm{MgSO}_{4}$ and $\left.1.5 \mathrm{~g} \mathrm{NaOAc}\right)$ had poorest recovery.

2) $\mathrm{EN}$ method $\left(4 \mathrm{~g} \mathrm{MgSO}_{4}, 1 \mathrm{~g} \mathrm{NaCl}, 1 \mathrm{~g}\right.$ sodium citrate and $0.5 \mathrm{~g}$ sodium citrate sesquihydrate) had high matrix effects (signal enhancement) and poor chromatographic peak shape.

Using these results, a "mix and match" approach was devised; using the AOAC extraction tube with the dSPE kit following the EN method specification (900 $\mathrm{mg} \mathrm{MgSO}_{4}$ and $150 \mathrm{mg}$ PSA), as this formulation contained less $\mathrm{MgSO}_{4}$ and PSA, reducing the potential loss of compounds during the dSPE step by binding to the PSA sorbent. This method showed a positive impact on previous methods; chromatographic peak shape improved for those compounds previously displaying peak broadening (see Figure 5.3) and challenging analytes, previously showing poor sensitivity (ibuprofen) was detected above the background noise. 

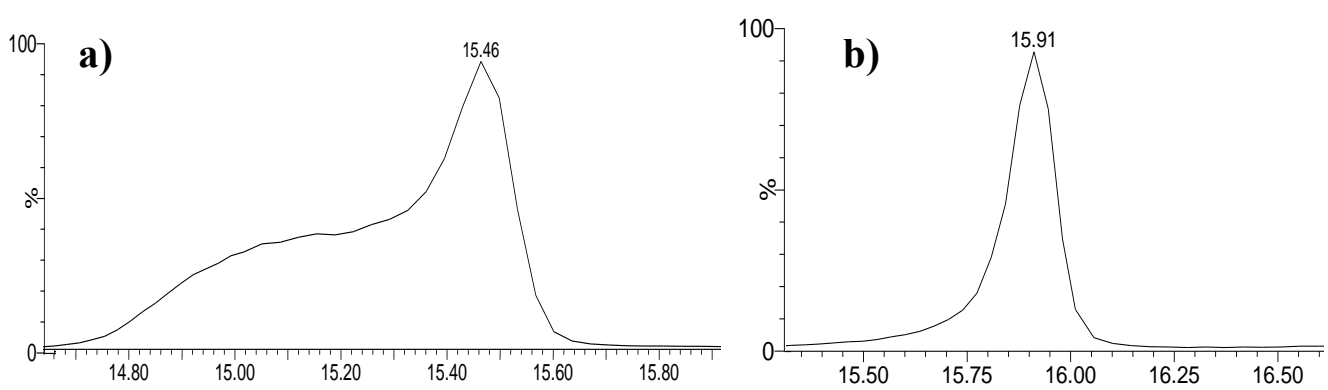

Figure 5.3: Chromatograms of propranolol under different extraction conditions to show the effect of $\mathrm{NaCl}$ on peak broadening; a) propranolol after extraction using the standardised EN QuEChERS method and b) the "mix and match" approach to QuEChERS extraction.

Despite the improvement in chromatographic peak shape, the mix and match method still showed significant matrix effects, with the majority of compounds exhibiting approximately 50-60\% ionisation suppression, assumed to be a result of the co-extraction of the QuEChERS kits. To minimise matrix effects a number of modifications were investigated including attempting to reduce the amount of polar co-extractives carried through within the acetonitrile supernatant by altering the amount of $\mathrm{MgSO}_{4}$ in the initial extraction and dSPE kits, and adding acetic acid to the extract before dSPE as described in Caldas et al. [9].

As diclofenac and ibuprofen were recovered using the AOAC method, but not with the EN method, the first approach involved increasing the amount of $\mathrm{MgSO}_{4}$ within the EN dSPE kit by $300 \mathrm{mg}$ to reflect the amount of drying agent in the AOAC method. The result showed improved repeatability for the matrix effects, with $\% \mathrm{RSD} \leq 10 \%$ however, there was no change in $\% \mathrm{ME}$ or $\% \mathrm{REC}$, with calculated average results of $52 \%$ and $44 \%$, respectively. As expected diclofenac and ibuprofen continued to be extracted using this method, while the $\% \mathrm{ME}$ remained unchanged at 40 and $42 \%$ respectively, the recovery for both compounds did increase slightly, with \%REC at $4 \%$ for diclofenac and $28 \%$ for ibuprofen. Despite the improvement to the acidic compounds, this extraction performance was less successful for the other compounds and therefore was not investigated further.

The second approach involved the addition of $1.0 \%$ acetic acid after the first centrifugation step, with the aim of increasing the recovery of the more acidic compounds. The volume added was determined by the amount of supernatant obtained from the initial extraction, for example, if $7 \mathrm{~mL}$ of supernatant was carried through to dSPE, $70 \mu \mathrm{L}$ of acetic acid was added. Similar to the previous approach, the matrix effects showed significant signal suppression, with an average of $48 \%$, however, the recovery of the compounds improved, particularly for the acidic compounds, diclofenac and ibuprofen, increasing to $20 \%$ and $30 \%$, respectively. The recovery of the basic compounds also increased slightly, with an average 
recovery of 53\%. These positive results showed that this method had potential to extract all pharmaceuticals in the suite.

The third approach involved the reduction of $\mathrm{MgSO}_{4}$ from the AOAC extraction kit to $4 \mathrm{~g}$ (as found in the EN kit) to determine whether loss of compound was occurring at this initial step due to this drying agent. This resulted in an increase in recovery of the basic compounds, with \%RECs of between $75-95 \%$. The matrix effects also improved, with an increasing percentage to an average of $53 \%(\% \mathrm{RSD}<10)$. As the second approach, with the addition of acetic acid gave the best recovery for the acidic compounds, a combined method of $4 \mathrm{~g}$ of $\mathrm{MgSO}_{4}+1.5 \mathrm{~g} \mathrm{NaOAc}$, with the addition of $1.0 \%$ acetic acid was also investigated, with the aim of assessing if good recovery of both the basic and acidic compounds could be acheived. However, this combination of extraction conditions caused an adverse effect on the chromatography, similar to that seen with the EN extraction method, so this method was not investigated further. Therefore, given the results of these modification experiments the third

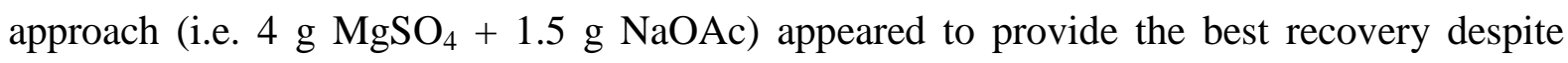
significant ionisation suppression being observed and was chosen for further study.

\subsubsection{2: Investigation of Alternative dSPE Sorbents}

Standardised QuEChERS methods include a variety of dSPE sorbents depending upon the composition of the sample matrix. For the initial method development, the dSPE step comprised of PSA and $\mathrm{MgSO}_{4}$, however for more complex samples such as soils and sludge, dSPE that includes C18 (used for removal of lipids) and GCB (used for removal of pigments/planar compounds) are readily available to tailor the extraction. Given these materials are capable of hydrophobic bonding it is likely that these may also remove the target pharmaceuticals as well as the interference, resulting in a decrease in recovery. To assess whether the inclusion of these sorbents had a positive effect on the matrix effects and recovery of pharmaceuticals these were investigated with the modified initial extraction it. The evaluation indicated that the presence of C18 adversely affected the chromatography of acetaminophen; usually a single peak at 1.56-1.60 minutes, however after extraction, an additional peak was seen, affecting the selectivity of the SIM transition (see Figure 5.4). A further drawback to this extraction was as expected, a decrease in recovery, despite relatively constant matrix effects being observed for each compound. This is understandable given most of these compounds (apart from acetaminophen and ibuprofen) showed significant retention on C18, albeit as an LC column. 


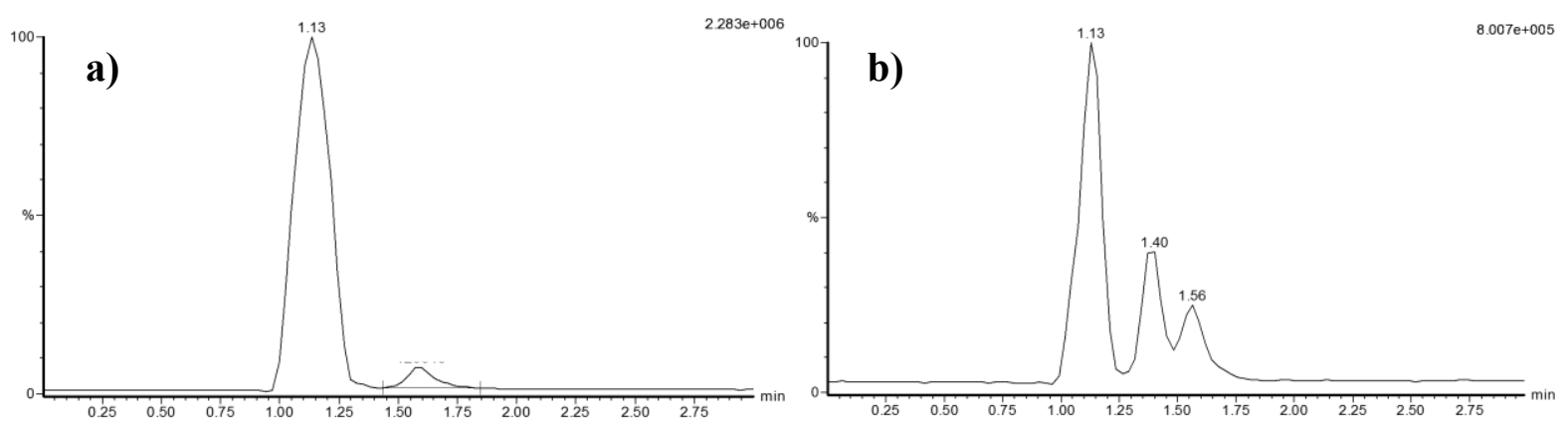

Figure 5.4: Chromatograms of acetaminophen using multiple dSPE extraction methods to show the effect of different sorbents; a) single peak seen using dSPE containing PSA and $\mathrm{MgSO}_{4}$ only and b) split peaks seen using dSPE containing PSA, $\mathrm{MgSO}_{4}$ and C18.

However, the dSPE containing PSA, $\mathrm{MgSO}_{4}$ and GCB (pigmented) showed improved matrix effects and recovery results (comparable to the PSA only dSPE) over the extraction using C18 (see Appendix 5.3). This indicates that the interaction with the target pharmaceuticals is less, and equivalent to the PSA sorbent. Although the matrix effects were still low, this equivalent recovery is a positive as the inclusion of GCB could be key in extracting some of the interferences expected when analysing more complex matrices such as fulvic acids within soil/sludge. However for further investigations into improving matrix effects, the dSPE kit containing PSA only was chosen to ensure comparability between previous experiments.

\subsubsection{3: Optimisation of Custom QuEChERS Extraction}

The modification of the QuEChERS extraction has led to the development of a repeatable method with apparent recovery (albeit to differing amounts) of all analytes however, a significant amount of ionisation suppression was observed in each experiment. This is important to address as the matrix effects can influence the recovery measurement. To investigate ways to improve the matrix effects, the modified extraction that provided the highest recoveries (i.e. $4 \mathrm{~g} \mathrm{MgSO}_{4}+1.5 \mathrm{~g} \mathrm{NaOAc}$ ), was used. As changes to the QuEChERS protocol had been investigated, focus shifted to the initial pharmaceutical spike. Previous experiments have used small volumes $(20 \mu \mathrm{L})$ of highly concentrated pharmaceutical and IS mixtures $(1 \mu \mathrm{g} / \mathrm{mL})$ spiked into the water sample before extraction. It was considered whether this high concentration mixture was contributing to the matrix suppression with the pharmaceuticals influencing the signals observed and suppressing each other. Therefore a less concentrated spike was investigated, both as a spike of a separate analyte and IS mixture and, as the concentration of the pharmaceutical and IS spikes were the same (100 ng/mL), a more efficient, single standard mixture, with a total spike volume of $500 \mu \mathrm{L}$. Sadly, the latter 
approach gave an overall poor result; \% ME improved versus the $40 \mu \mathrm{L}$ spike, but ionisation enhancement was observed with values of $83-198 \%$, and poor repeatability of $32-132 \%$ RSD The single spike also resulted in poor peak shape for pronethalol and carbamazepine, confirming that the analytes can influence each other's observed signal and the combination of the pharmaceuticals and IS suite in a single mixture is not compatible with the extraction. The experiment was therefore repeated using separate mixtures of pharmaceuticals and IS, ensuring the same starting concentrations $(100 \mathrm{ng} / \mathrm{mL})$, with an initial spike volume of 250 $\mu \mathrm{L}$ of each, resulting in $500 \mu \mathrm{L}$ volume overall. This proved critical reducing the matrix effects significantly with \%ME between $95-125 \%$ for all compounds, with the exception of acetaminophen-(methyl) $-\mathrm{d}_{3}$ which had an enhanced signal of $164 \%$ ME, possibly due to its elution near the solvent front. This approach also showed excellent repeatability, with \%RSD $\leq 20 \%$ for all compounds and was selected as the optimised QuEChERS extraction for further investigation, and will be referred to henceforth as the modified QuEChERS method.

\section{2: QuEChERS Extraction with Solid-Phase Extraction}

As the sample preparation methods adopted by industry all incorporate traditional solid-phase extraction (SPE) using cartridges/columns, it was proposed whether a mix of QuEChERS extraction and cartridge SPE could provide improved sample clean-up than the modified QuEChERS method as analyte extraction would be more targeted. To determine this, a variety of different SPE cartridges were investigated for analyte recovery.

\subsection{1: Investigation of Commercial SPE Cartridges}

Cartridge SPE is a highly versatile extraction process capable of operating to both selectively extract (retain) the target analytes for enhanced selectivity and high recovery or alternatively, to specifically extract a certain type of interference to provide a sample extract that is complete as possible for screening (i.e. dSPE approach). Given this, the choice of sorbent (and elution solvent) is particularly important to meet the objective of the extraction. For the pharmaceutical extraction, both approaches were tested using commercial SPE cartridges, supplied by Biotage; ISOLUTE® ENV+ and ISOLUTE® SCX-2, with the aim of assessing retention and removal of salt to reduce the matrix enhancement observed, respectively. 


\subsubsection{1: ISOLUTE® ENV+}

ISOLUTE® ENV+ is a hyper crosslinked hydroxylated polystyrene-divinylbenzene copolymer, designed to extract polar analytes from water samples. As these cartridges were tested in place of the dSPE step in the QuEChERS protocol, an additional evaporation and solvent exchange step was needed after the initial extraction step, to have an aqueous loading solution to ensure analyte retention on the hydrophobic sorbent. After loading the sample onto the cartridge, analytes were eluted using acetonitrile and, as with the QuEChERS extractions were evaporated to dryness for solvent exchange into the mobile phases (50:50 water/acetonitrile). However, it was found that the solvent evaporation took considerably longer than expected (approximately 4 hours for $4 \mathrm{~mL}$ ), possibly due to co-extraction of water from the cartridge. Given these time implications, it was thought that this procedure would not be suitable for high throughput analyses and was not investigated further.

\subsubsection{2: ISOLUTE® $S C X-2$}

ISOLUTE® SCX-2 is a strong cation exchange sorbent with minimal non-polar character, used to extract basic analytes from aqueous samples. These cartridges were also tested with the environmental matrix in mind, as a potential method to retain interferences common to soil and sludge [10-12] for removal. The QuEChERS extraction supernatant was loaded onto the cartridge and the eluent collected for analysis. However, it was found that the interaction between the sorbent and the suite of pharmaceuticals was too strong as each one was retained on the cartridge, so was not investigated further.

\subsection{2: Investigation of Cartridge-dSPE}

Following on from the "mix and match" approach in the development of the QuEChERS protocol we also tested the efficacy of cartridges filled with the QuEChERS dSPE material. This initially considered a $3 \mathrm{~mL}$ cartridge filled with PSA and $\mathrm{MgSO}_{4}$ however, was deemed impractical as the sorbent filled the majority of the cartridge, leaving space to add approximately $1 \mathrm{~mL}$ of supernatant. This meant that it took an overly lengthy period of time for the supernatant to permeate the sorbent, further exacerbated by the typical volume of supernatant collected from the QuEChERS extraction $(7-8 \mathrm{~mL})$. The results showed that the pharmaceuticals also showed poor recovery, possibly due poor solvent recovery and other cartridge sizes were considered. A $6 \mathrm{~mL}$ and a $15 \mathrm{~mL}$ cartridge were then investigated and both worked well with all pharmaceuticals extracted. When compared with the optimised 
QuEChERS with dSPE method, the results obtained for the $15 \mathrm{~mL}$ cartridge were more competitive to those achieved using dSPE. For example, despite an increase in matrix enhancement over the entire suite (91-149\% ME) the \%REC was generally slightly lower the target compounds, apart from carbamazepine, 10,11-dihydrocarbamazepine and loratadine, which in fact increased by up to $17 \%$. These comparable results show that this method is viable for use within a laboratory set-up to undertaken routine, automated SPE. However, for the purpose of this study, the dSPE method was chosen for further investigation due to the overall better performance of traditional dSPE.

\section{3: Performance of Optimised QuEChERS Method in Water}

The modified QuEChERS method (4 $\mathrm{g}$ of $\mathrm{MgSO}_{4}$ with $1.5 \mathrm{~g} \mathrm{NaOAc}$ ), with dSPE containing $\mathrm{MgSO}_{4}$ and PSA showed minimal, repeatable matrix effects with results approximately $100 \%$ ME (see Figure 5.5), with $\%$ RSD $\leq 16 \%$ for all compounds. These results provide confidence that the recovery measurements for each target compound are a true representation of the extraction efficiency enabling a more accurate measurement of the amounts qualified in the target sample. When recovery was assessed the majority of the pharmaceuticals showed excellent repeatability with \%RSD $<15 \%$, apart from acetaminophen, which showed greater variability than the other compounds, with a \%REC of $92 \%$ and a $\% \mathrm{CV}$ of $20 \%$. This compound eluted very near the solvent front and this could have resulted in the variability of the signal.

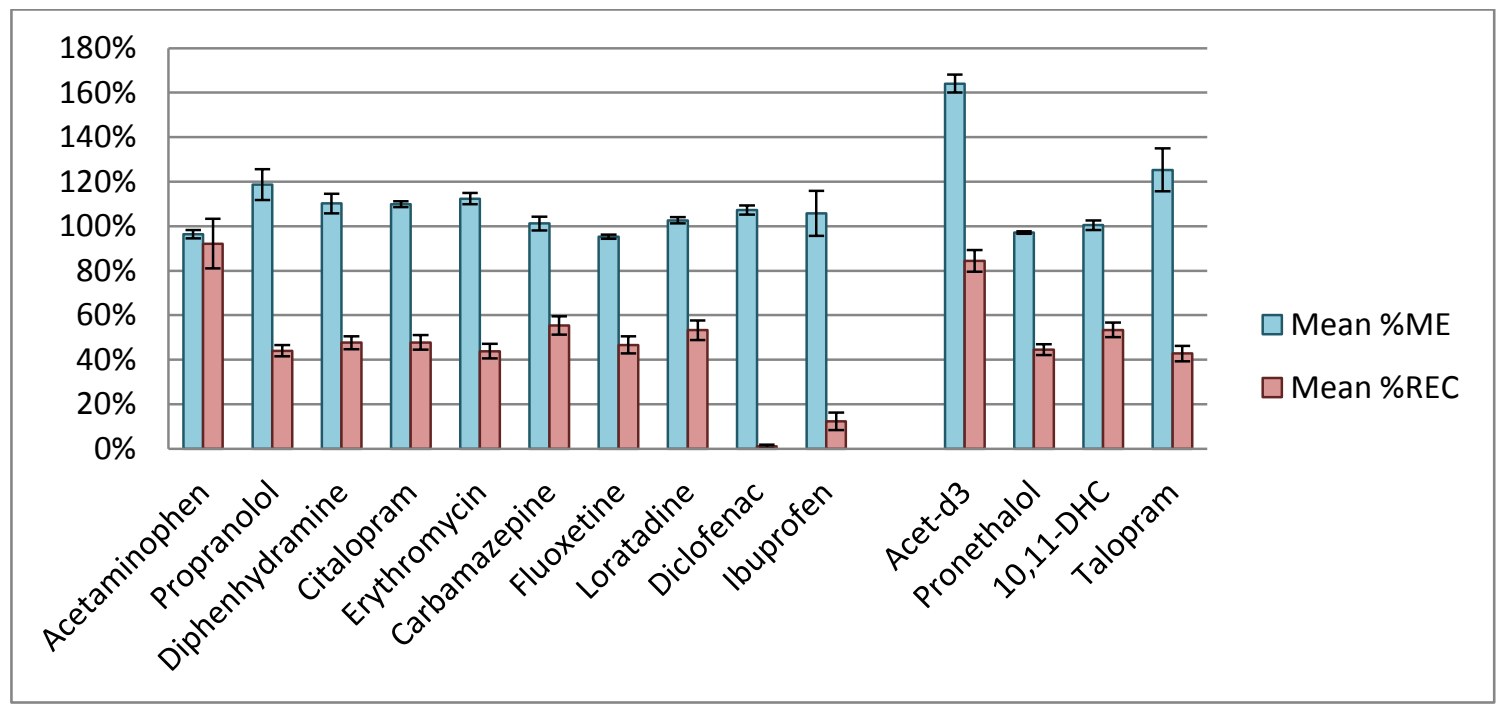

Figure 5.5: A summary of the percentage matrix effects and recovery for the entire suite of pharmaceuticals extracted from "ideal" water matrix using the modified QuEChERS extraction method. 
However, from a closer inspection of the data it appears that the method shows a more favourable recovery for basic compounds with typical recoveries between $42-55 \%$, while the acidic compounds showing poorer precision and recovery with a \%RD of 124 and 55\% and a \%REC of 1.1 and $12.4 \%$ and for diclofenac and ibuprofen respectively (see Figure 5.5). A potential cause of the poor recovery of acidic compounds may be the presence of primary secondary amine (PSA) within the QuEChERS dSPE kit as this sorbent has a permanent positive charge that could retain these compounds during this extraction step. One way to mitigate this may be to alter the dSPE material, opting for an anion exchange sorbent, rather than PSA, however, this is likely to affect the basic compounds and could result in further matrix suppression with complex matrices, such as sludge that have a high fulvic and humic acid content. Based on this premise the method was not optimised further on this sample type but applied to a more complex soil matrix.

\section{4: Performance of Optimised QuEChERS Method in Soil}

The modified QuEChERS extraction method was tested using locally sourced garden soil as a control matrix for treated sludgecake. The soil was fortified with pharmaceutical and IS mixtures to determine the \% ME and \%REC in a more complex sample matrix. As expected, the $\% \mathrm{ME}$ for each compound altered slightly versus the "ideal" water samples, with highly repeatable results for all compounds (8\% RSD) and the majority of compounds ranging from 90-155\% ME, with a median value of $107 \%$. The higher \% ME observed were for the compounds that eluted in the middle of the chromatographic gradient (propranolol, diphenhydramine, citalopram and erythromycin at 139\%, $150 \% 155 \% 131 \% \mathrm{ME}$, respectively), and is likely to be a result of co-elution of interferences (in particular, salts and heavy metals [10]) within the soil with a significant number of peaks apparent in the full mass scan chromatogram. 


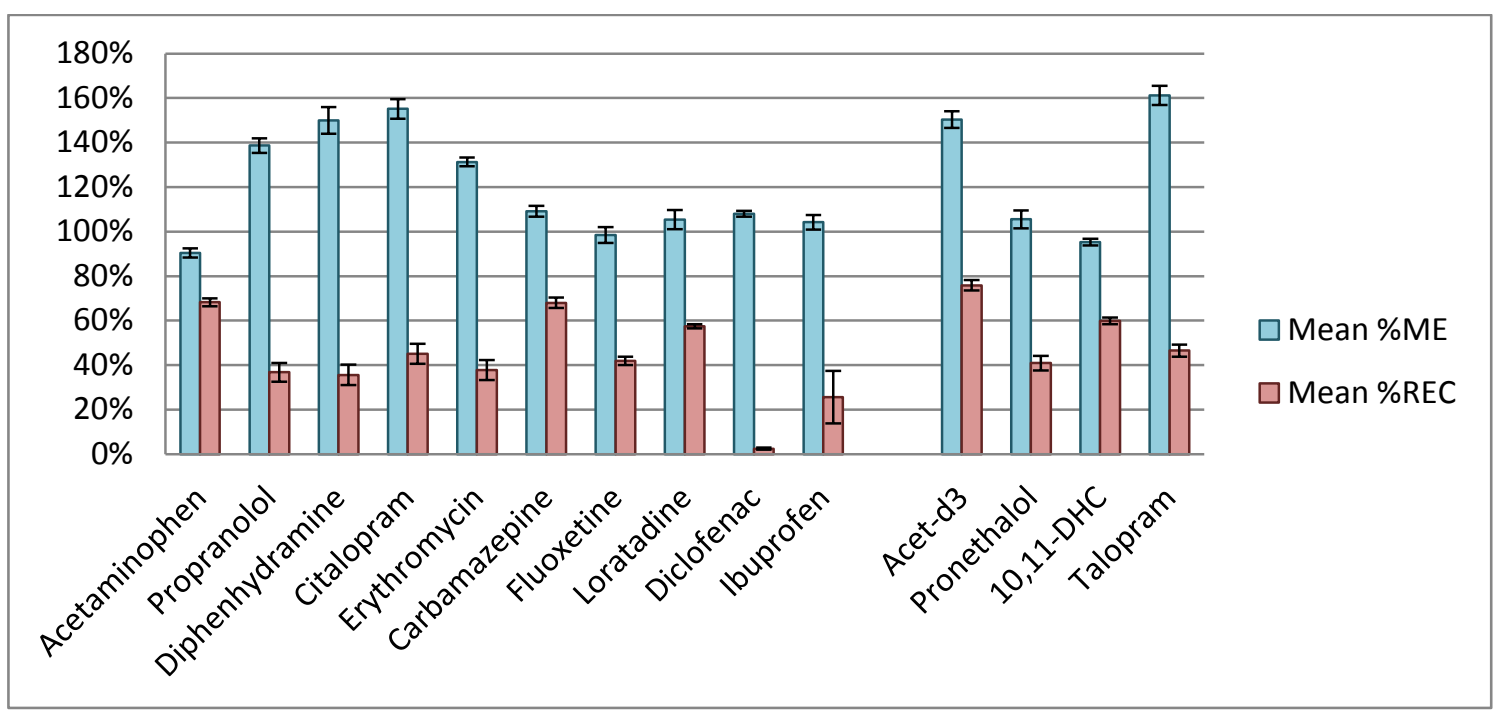

Figure 5.6: A summary of the percentage matrix effects and recovery for the entire suite of pharmaceuticals extracted from fortified soil matrix using the modified QuEChERS extraction method.

The IS, talopram exhibited the highest amount of matrix enhancement, with a \%ME of $161 \%$. As with the solvent sample, recovery of diclofenac and ibuprofen was poor at $2 \%$ and $25 \%$ respectively, further supporting the idea that retention of compound on the dSPE sorbent occurred. However, more pleasingly only a slight decrease in analyte recovery (35-75\%) and precision $(\% \mathrm{RSD} \leq 22 \%)$ was seen across the remaining suite of pharmaceuticals which, given the increase in \%ME would potentially indicate that recovery is in fact a little higher than these values.

\subsubsection{Comparison Study with Industrial Method}

To benchmark the modified QuEChERS method this was compared to the recognised methods used within environmental monitoring. Fortified soil samples were taken through the modified QuEChERS protocol and the method used by Natural Resources Wales (NRW) [2] (initially developed for use on treated sludgecake) and analysed. Although the NRW method specifically targets a small selection of the pharmaceuticals within this study (propranolol, erythromycin and fluoxetine), identical spiking solutions were used for both methods (i.e. $250 \mu \mathrm{L}$ of $200 \mathrm{ng} / \mathrm{mL}$ pharmaceutical and IS mixtures). The sample preparation involves repeated solvent washings of $50 \mathrm{mg}$ of starting material (soil), which is collected in one vial to give an acidic and a basic supernatant. In both fractions, not one of the compounds spiked at the start of the extraction were detected; potentially lost during sample dilution, thus demonstrating that the modified QuEChERS method developed is a more successful method for the extraction of pharmaceuticals from soil. 
The EPA Method (1694) for the analysis of water, soil, sediment, and biosolids [1] is a multistep analysis involving solid-liquid extraction, evaporation and $\mathrm{pH}$ adjustment before separation into acidic and basic SPE fractions for further clean-up before LC-MS/MS analysis. The latter stage of this method was evaluated using a spiked water sample to test the $\% \mathrm{ME}$ and \%REC of the Oasis HLB SPE cartridge for the target compounds. Pleasingly, all pharmaceuticals were detected in both the acidic and the basic fractions however, the results showed significant ionisation suppression, with the median $\% \mathrm{ME}$ of $39 \%(\% \mathrm{RSD} \leq 10)$. The recovery values showed poor reproducibility, with \%RSD of 10-129\% (median of 46\%), further demonstrating that the modified QuEChERS method is more successful for the extraction of pharmaceuticals and also offers a labour-saving and cost effective approach for high throughput analysis versus current protocols. It is estimated that extraction costs can be reduced by $>60 \%$ solely from the extraction cartridges and further still with analyst time saved, from hours to approximately 20 minutes per sample.

\section{5: Application of Optimised QuEChERS Method: Pharmaceuticals and Biocides}

The optimised QuEChERS method was evaluated for the combined extraction of pharmaceuticals and biocides, to replicate the extraction conditions anticipated within sludge samples. An in-house pilot study showed the presence of biocides in high abundance within sludgecake samples, therefore any effect on matrix suppression or enhancement and recovery of pharmaceuticals due to the presence of the biocides needed to be established, in addition to the effectiveness of this optimised method on the extraction of biocides for quantitation.

\subsection{1: Performance of Optimised QuEChERS Method in Water}

The optimised QuEChERS sample preparation method was tested in water to establish whether it could be used for the simultaneous extraction of pharmaceuticals and biocides as the presence of biocides within sludge samples could be of interest to CIP. Previous tests with QuEChERS have shown that the biocides can be carried through the extraction process; therefore it needs to be established what, if any, effects were observed by the presence of both compound classes (i.e. matrix interference/reduction in recovery) to ensure the accurate quantitation of the target analyte in more complex matrices. The QuEChERS method was carried out using a spiking mixture of the pharmaceuticals and biocides, as described in Section 2.4.4 and analysed using both analytical methods. While the \%RSD for each 
pharmaceutical was calculated to be $15 \%$, indicating good reliability of the data, the results indicate that the matrix effect for each pharmaceutical was impacted by the presence of biocide within the extract, despite their later elution off the column, with the majority exhibiting a slight degree of enhancement (median value increasing from $106 \%$ to $109 \%$ ). The exceptions to this are acetaminophen and erythromycin as both showed slight suppression compared to the pharmaceutical only extraction at $90 \%$ and $109 \%$ respectively, which could lead to a slight underestimation of concentration within sludgecake if $\% \mathrm{ME}$ is not taken into account. Acetaminophen-(methyl $)-\mathrm{d}_{3}$, pronethalol and loratadine however, appeared to be unaffected by the biocides, with matrix effect remaining the same at $164 \%$, $97 \%$ and $102 \%$ respectively. The greatest difference observed was for ibuprofen, the only compound analysed in negative ion mode; the \% ME increased from $105 \%$ to $173 \%$.

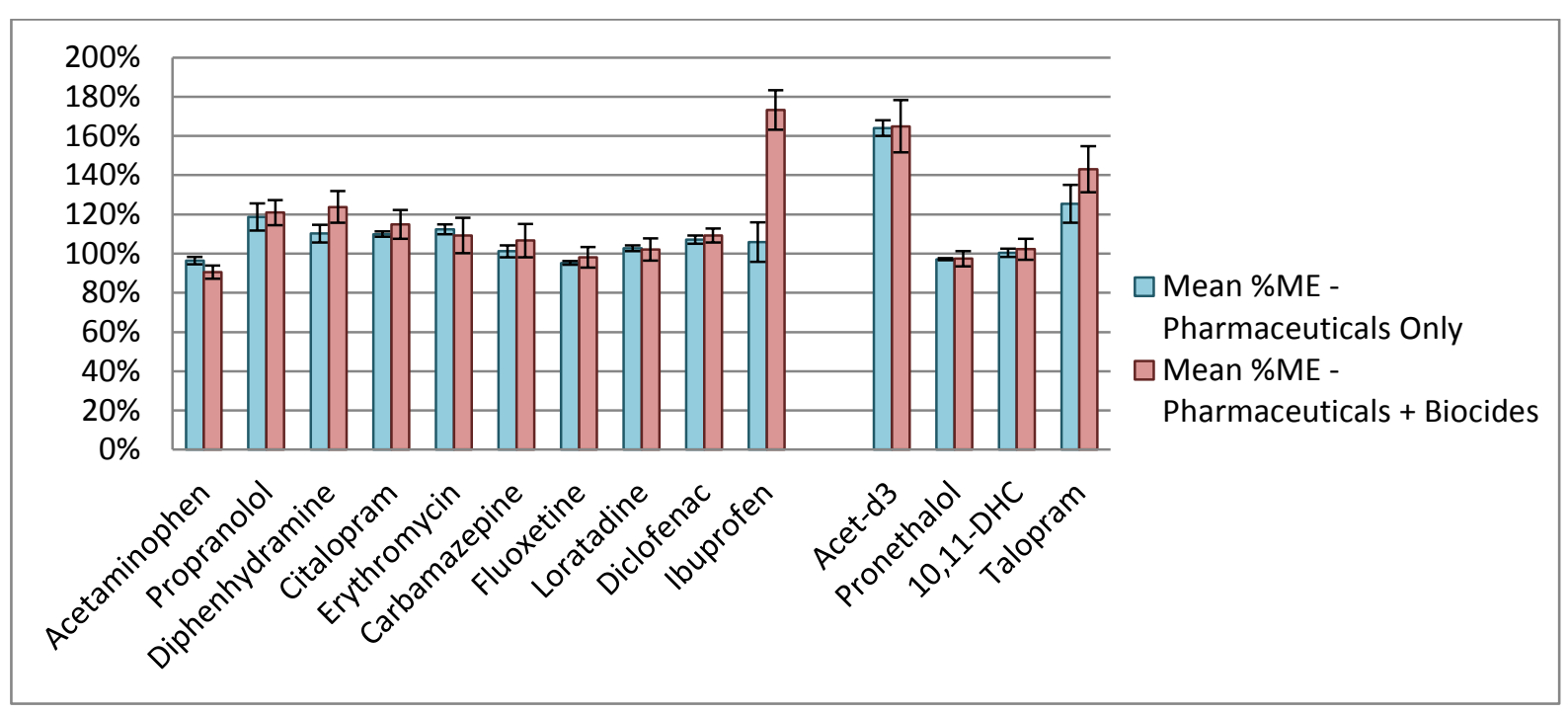

Figure 5.7: A comparison summary of the percentage matrix effects for the entire suite of pharmaceuticals in the presence of biocides, extracted from "ideal" water matrix using the modified QuEChERS extraction method.

The suite of biocides showed repeatable matrix effects $(\% \mathrm{RSD} \leq 16 \%$, except DDMA where $\%$ RSD $=25 \%$ ), with marginal impact of the signal with a median value of $99 \%$. As the biocides are permanently charged salts, they are less prone to competitive ionisation through protonation (i.e. as with the pharmaceuticals), therefore the minimal matrix effects observed is expected. The highest matrix effect observed was for HDTMA at 124\%; this may be due to co-eluting species as HDTMA elutes off column similar retention time as BAC-C14 and the IS BAC-C14- $\mathrm{d}_{7}$, and from close inspection of the data, a large peak was recorded within the SIM window for HDTMA at 35 minutes. This peak had a similar $m / z$, at 282 and as this peak is not observed within the QC samples, it is likely that this interference originated from the 
QuEChERS extraction sorbents. These results provide confidence that the recovery measurements for each compound are a true representation of the extraction efficiency.

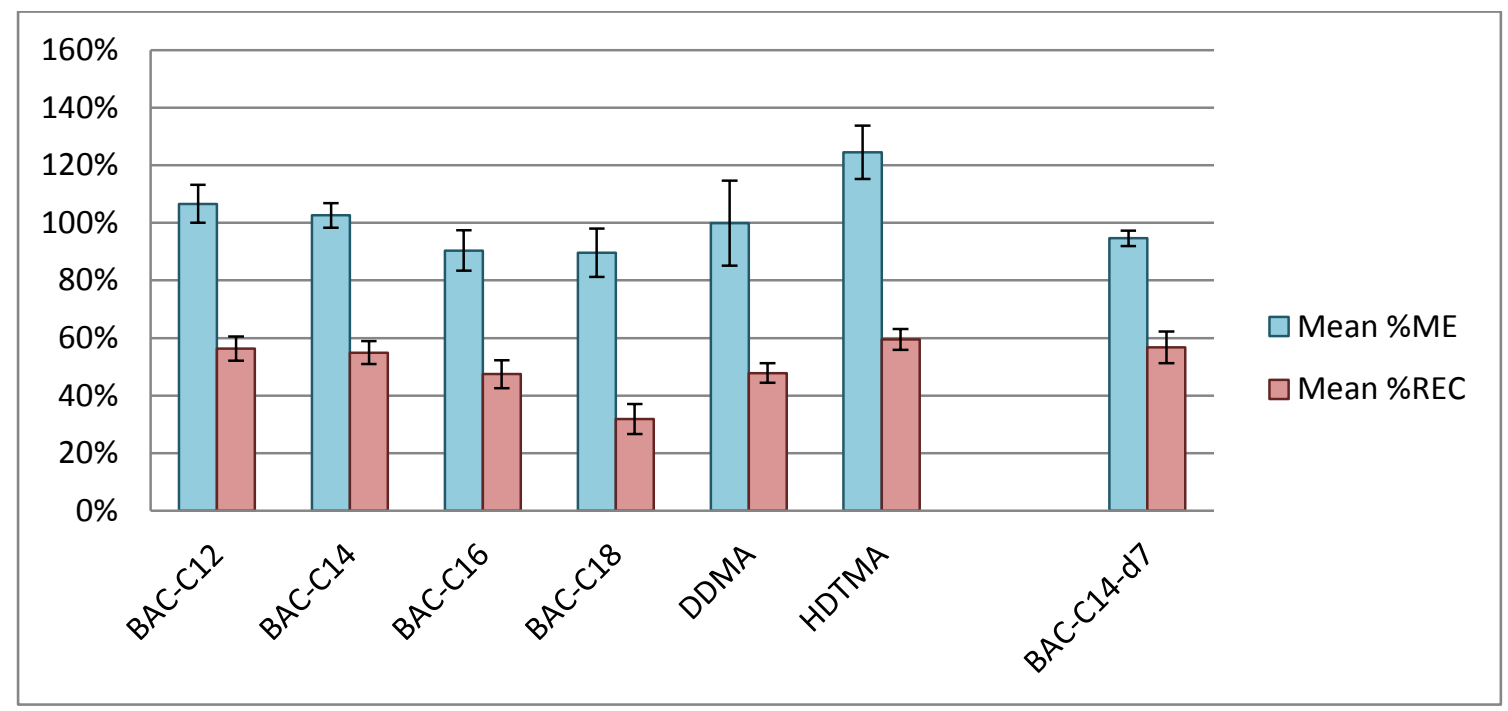

Figure 5.8: A summary of the percentage matrix effects and recovery for the entire suite of biocides extracted from "ideal" water matrix using the modified QuEChERS extraction method.

The recovery measurements were also assessed, and while the biocides had good, repeatable recovery $(\% \mathrm{RSD} \leq 17 \%$, except BAC-C18 where $\% \mathrm{RSD}=28 \%)$ of approximately $50 \%$ for each compound (i.e. $31 \%$ for BAC-C18 at the lowest, and HDTMA at 59\% as the highest), all of the pharmaceuticals saw a decrease in recovery, with the exception of the acidic compounds, diclofenac and ibuprofen. The increase seen for these compounds could be explained by the increase presence of free chloride/bromide ions from the biocides competing for the free active sites on the dSPE material, resulting in less compound retention. 


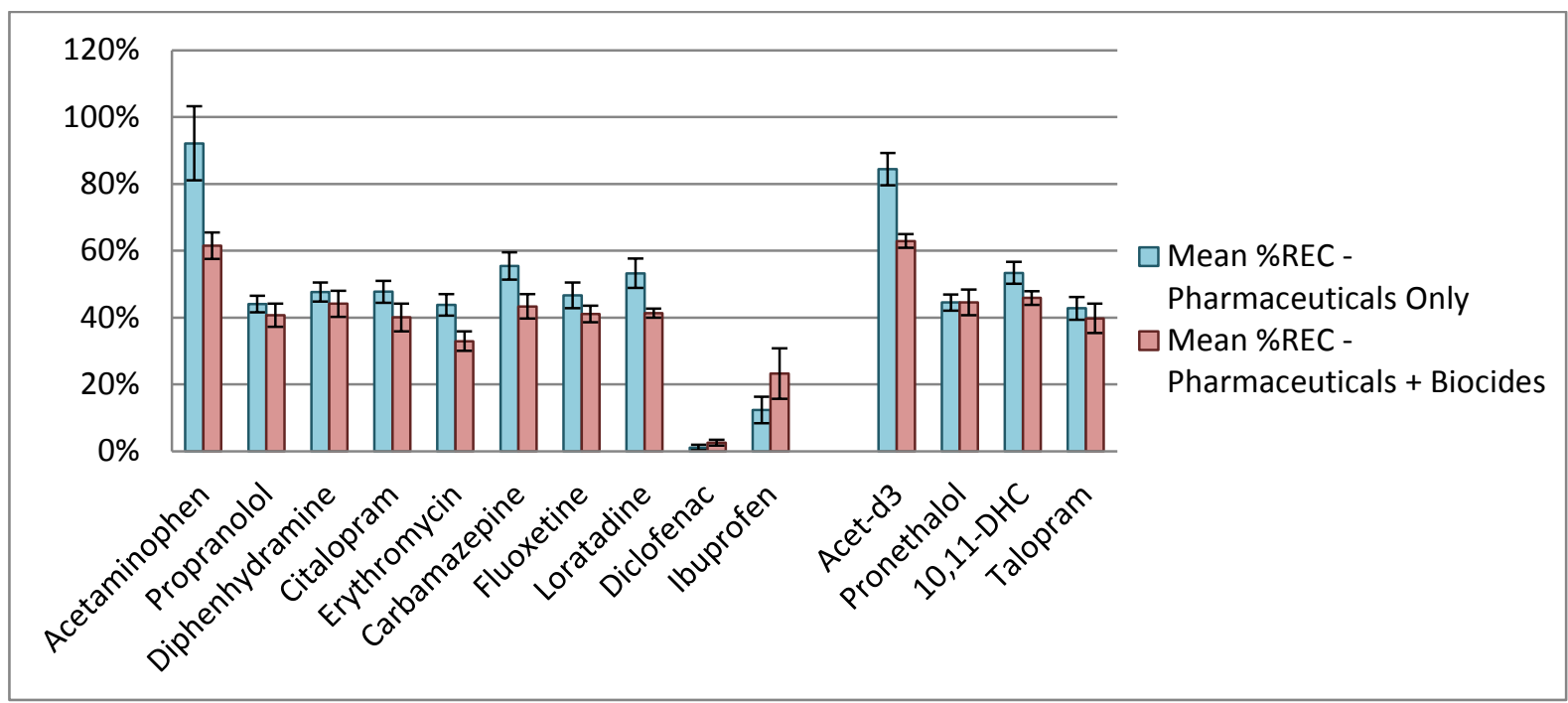

Figure 5.9: A comparison summary of the percentage recovery for the entire suite of pharmaceuticals in the presence of biocides, extracted from "ideal" water matrix using the modified QuEChERS extraction method.

\subsection{2: Performance of Optimised QuEChERS Method in Soil}

Similar to the pharmaceutical only study detailed in Section5.4, the QuEChERS sample preparation method was evaluated for the extraction of pharmaceuticals and biocides using locally-sourced garden soil as a control matrix. Testing this sample matrix with the biocides is particularly useful to determine the method's ability in monitoring for these compounds for antimicrobial resistance studies. When comparing the matrix effects for the pharmaceutical only study and this extraction, a decrease in enhancement was seen (see Appendix 5.5). For example, the pharmaceutical only study showed a \%ME of $90-155 \%$ (median value of $107 \%$ and \%RSD <8\%), with the highest \%ME observed for propranolol, diphenhydramine, citalopram and erythromycin $(139 \%, 150 \% 155 \% 131 \%$, respectively), all of which elute between 15-17 minutes. However, when the biocides were included within the spiking mixture, these measurements decreased to $125 \%, 133 \% 136 \%, 123 \%$ respectively. The $\% \mathrm{ME}$ for this extraction was between $91-141 \%$, with a median value calculated to be $112 \%$ and $\%$ RSD $\leq 10 \%$, with the exception of erythromycin and ibuprofen, with $\%$ RSD of $22 \%$ and $26 \%$, respectively. 


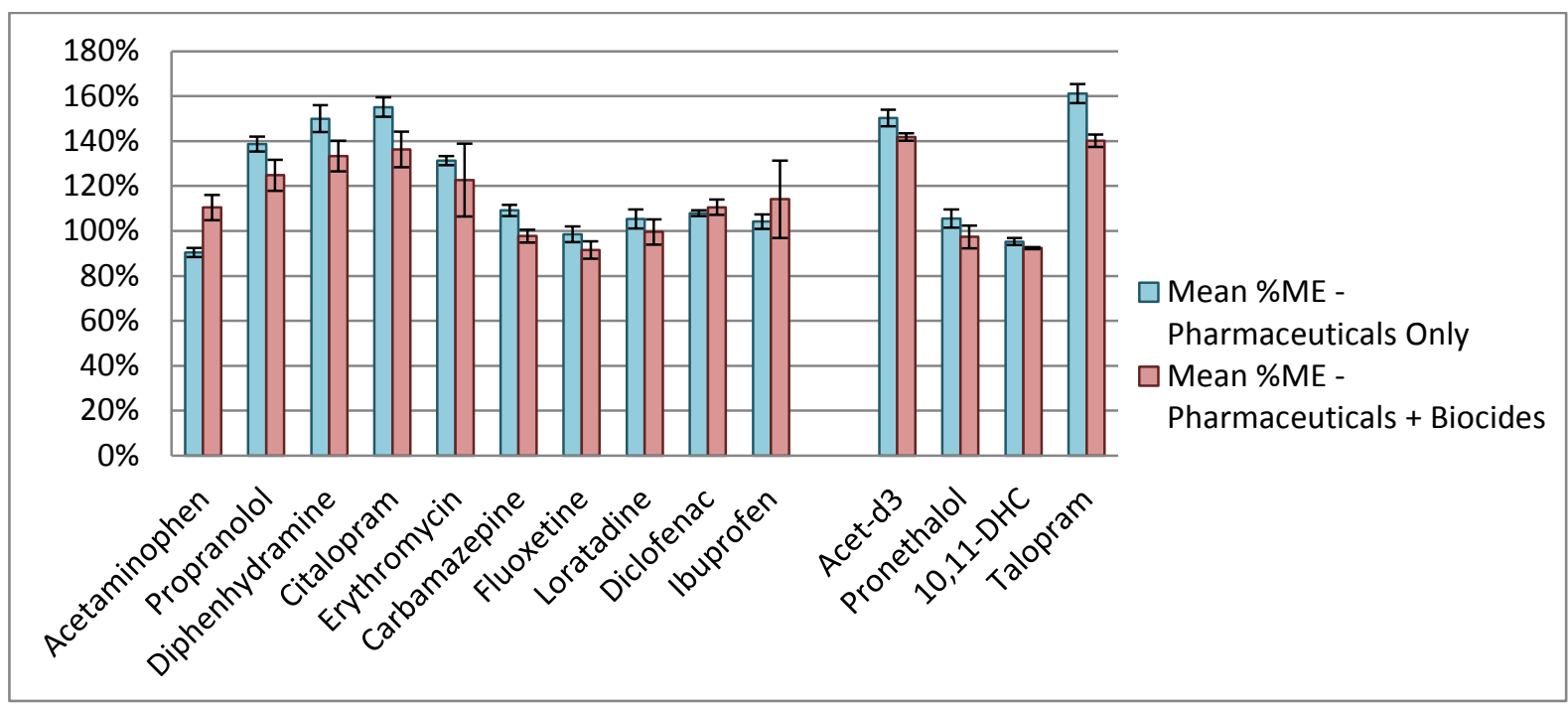

Figure 5.10: A comparison summary of the percentage matrix effects for the entire suite of pharmaceuticals in the presence of biocides, extracted from fortified soil matrix using the modified QuEChERS extraction method.

The majority of the pharmaceuticals saw a slight increase in recovery, with a range of 39$100 \%(\% \mathrm{RSD} \leq 17 \%)$, giving a median of $54 \%$ versus the pharmaceutical only extraction. The exception to this was the acidic compounds, diclofenac and ibuprofen, where a decrease in $\%$ REC were observed, as well as high \%RSD at $52 \%$ and $36 \%$ respectively, indicating that the extraction of these compounds from soil and more complex matrices may not be repeatable, with a significant risk of underestimating concentrations of these target analytes within the environment.

\subsubsection{1: 1:400 Dilution for Biocides}

From a previous in-house study, it was observed that biocides were detected in high abundance within treated sludgecake, therefore a dilution factor was added to ensure the extracts could be quantified within the concentration range used using the ion trap platform. A $2.5 \mathrm{~g}$ sample of soil was fortified with the spiking solution and taken through the QuEChERS protocol, reconstituted as standard before being diluted to 1:400 with a 50:50 acetonitrile/water mixture. The suite of biocides showed a slight increase in matrix effects versus the water extraction, with a range of $108-115 \%$, giving a median value of $111 \%$. While a high percentage of matrix enhancement was seen for HDTMA in water (124\%), this enhancement was reduced when extracted from soil (111\%) and may be due to the dilution of the sample as this is a common method used to reduce matrix effects within samples. For example, dilution enables the reduction of a less detectable level of co-eluting species that are causing enhancement. 


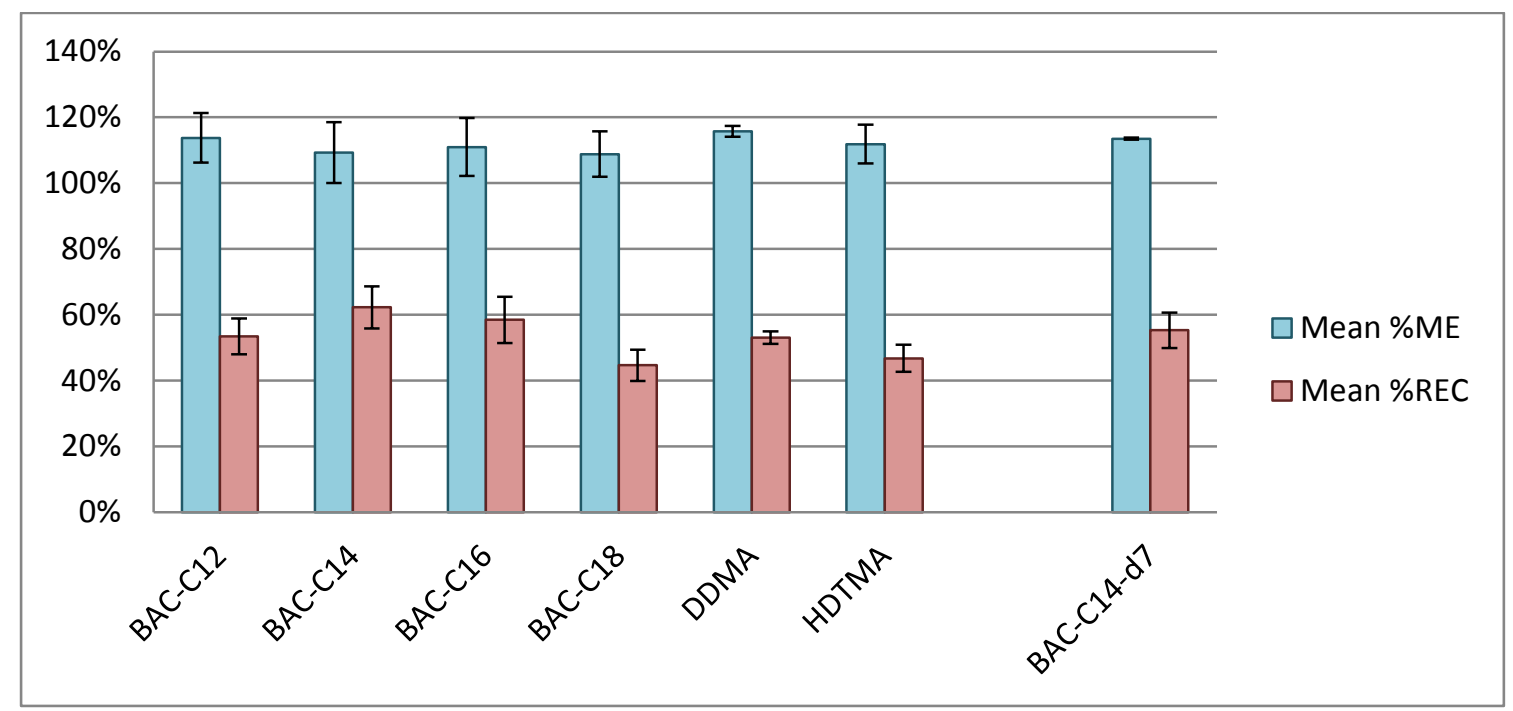

Figure 5.11: A summary of the percentage matrix effects and recovery for the entire suite of biocides extracted from fortified soil matrix using the modified QuEChERS extraction method.

The recovery measurements observed in the soil experiment were similar to those seen within water; the biocides showed repeatable recovery (\%RSD $\leq 20 \%)$ with $\%$ REC remaining around $50 \%$ for each compound (i.e. $44 \%$ for BAC-C18 at the lowest, and BAC-C14 at $62 \%$ as the highest).

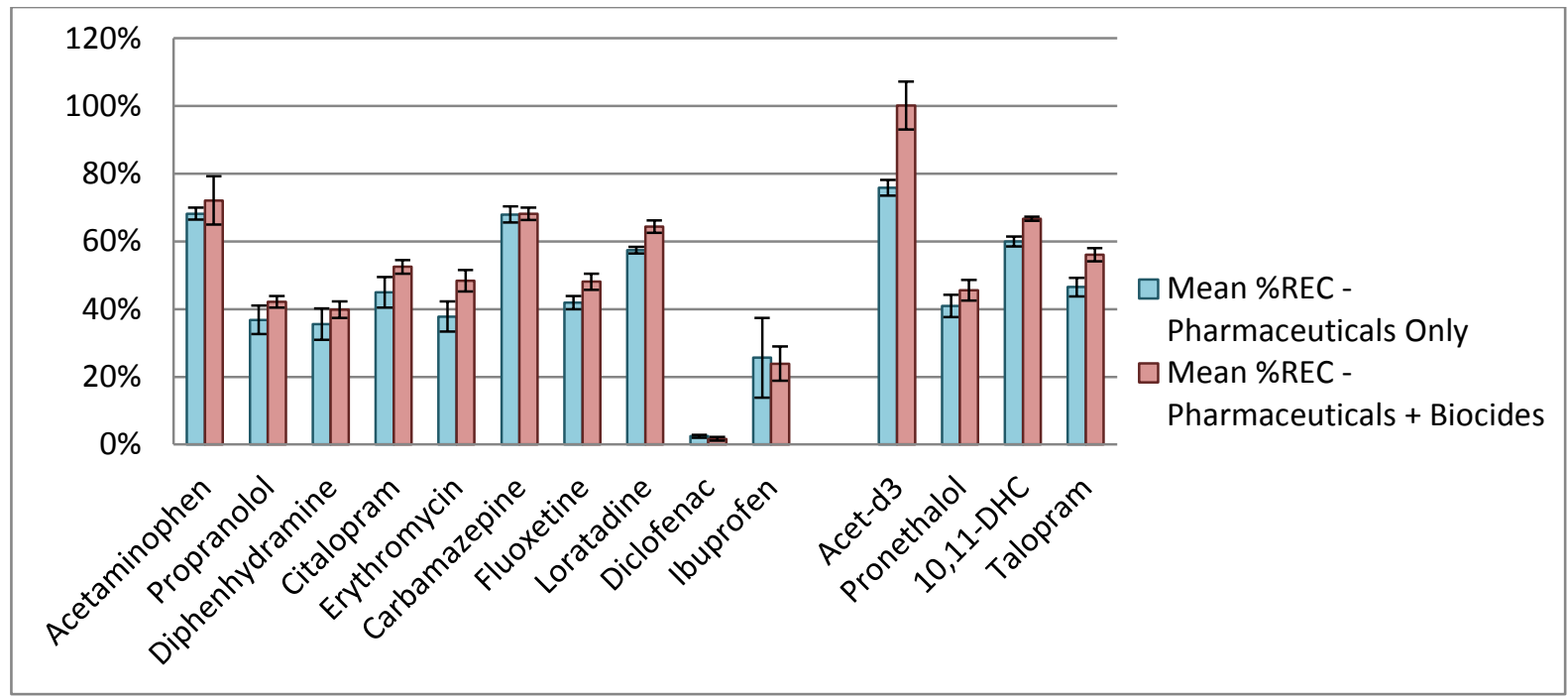

Figure 5.12: A comparison summary of the percentage recovery for the entire suite of pharmaceuticals in the presence of biocides, extracted from fortified soil matrix using the modified QuEChERS extraction method. 


\section{6: Conclusion}

Environmental pollution is a growing concern, with current research methods laborious, timeconsuming and often ineffective at sufficiently preparing complex matrices for extraction for potential compounds of interest. The aim of this research is to develop an alternative sample preparation method that is quick and simple to carry out, while providing sufficient sample clean-up for reproducible matrix elimination and recovery of target pharmaceuticals with little to no matrix effects. A modification to the QuEChERS sample preparation method for pharmaceutical extraction was investigated, which has successfully been applied to water and fortified soil samples, with the aim of future use for monitoring contamination in locally sourced treated sludgecake. Results showed that this method can be used to extract pharmaceuticals quickly from both water and soil with excellent repeatability and minimal matrix effects, providing confidence that the recovery measurements observed are an accurate representation of process (extraction) efficiency. While recovery of certain pharmaceuticals is lower than expected, this particular QuEChERS method works well for more basic compounds found on the CIP II list and could be a cost-effective alternative to the current industry recognised methods for monitoring the sites of waste deposition. This method has also shown potential for extracting these analytes from samples of high lipophilicity/organic content and could offer a more timely protocol for screening wastewater treatment processes to inform the CIP programme and future environmental policy. 


\section{References}

[1] Environmental Protection Agency. Pharmaceuticals and personal care products in water, soil, sediment and biosolids by HPLC/MS/MS. Washington, USA: EPA; 2007. Method 1694.

[2] Davies J, Schumacher M, Gazzard D. The determination of ofloxacin, oxytetracycline, propranolol, erythromycin and fluoxetine in sludge by liquid chromatography tandem triple quadrupole mass spectrometry using online SPE enrichment. Environment Agency Method of Analysis Suite One - Sludge. 2010: 1-12

[3] Anastassiades M, Scherbaum E, Taşdelen B, Štajnbaher D. Recent Developments in QuEChERS Methodology for Pesticide Multiresidue Analysis. In: Ohkawa H, Miyagawa H, Lee P W. editors. Pesticide Chemistry: Crop Protection, Public Health, Environmental Safety. Germany: Wiley-VCH; 2007.

[4] Townsend R, Desbrow C, Godfrey AR. Extraction of over-the-counter pharmaceuticals from water and sludge cake using ISOLUTE® QuEChERS prior to LC/MS analysis. Biotage, 2014. Report No.: AN831 Available from: http://www.biotage.com/news/clean-up-ofpharmaceuticals-in-sludge-cake

[5] Anastassiades M, Lehotay SJ. Fast and easy multiresidue method employing acetonitrile extraction/partition and "dispersive solid-phase extraction" for the determination of pesticide residues in produce. Journal of AOAC International. 2003; 86 (2): 412-431.

[6] Lehotay SJ. Determination of Pesticide Residues in Foods by Acetonitrile Extraction and Partitioning with Magnesium Sulfate: Collaborative Study. Journal of AOAC International. 2007; 90(2) 485- 520.

[7] Matuszewski BK Constanzer ML, Chavez-Eng CM. Strategies for the assessment of matrix effect in quantitative bioanalytical methods based on HPLC - MS/MS. Analytical Chemistry. 2003; 75 (13): 3019-3030.

[8] Chuang Y, Zhang Y, Wei Zhang W, Stephen A. Boyd SA, Li H. Comparison of accelerated solvent extraction and quick, easy, cheap, effective, rugged and safe method for extraction and determination of pharmaceuticals in vegetables. Journal of Chromatography A. 2015; 1404:1-9.

[9] Caldas SS, Bolzan CM, Cerquiera MB, Tomasini D, Furlong EB, Fagundes C, Primel EG. Evaluation of a Modified QuEChERS Extraction of Multiple Classes of Pesticides from a Rice Paddy Soil by LC-APCI-MS/MS. Journal of Agricultural and Food Chemistry. 2011; 59: 11918-11926.

[10] Giller KE, Witter E, McGrath SP. Heavy metals and soil microbes. Soil Biology \& Biochemistry. 2009; 41: 2031-2037.

[11] Adani F, Tambone F. Long-term effect of sewage sludge application on soil humic acids. Chemosphere. 2005; 60: 1214-1221. 
[12] Réveillé V, Mansuy L, Jardé E, Garnier-Sillama E. Characterisation of sewage sludge-derived organic matter: lipids and humic acids. Organic Geochemistry. 2003; 34: 615-627. 


\section{Chapter 6: Method Application: Complex Matrices}

Following positive results of the proof-of-concept work extracting pharmaceuticals from solid samples (i.e. soil) using the modified QuEChERS sample preparation method, the method was applied on other environmental samples; locally-sourced wastewater effluent, treated sludgecake and homogenised mussel tissue. These sample types were chosen to meet the demand of the amended Environmental Quality Standards Directive (EQSD) [1], where Member States should aim to collect data for priority substances specifically sediment and biota for a reliable long-term evaluation of the accumulation of these substances [1].

\section{1: Quantitative Analysis of Environmental Matrices}

A quantitative study was conducted for both pharmaceuticals and biocides using a dual mass spectrometry approach; an ion-trap and a quadrupole platform due to the different sensitivities required for the target pharmaceuticals and biocides. The samples were extracted simultaneously to ensure consistent conditions and analysed with a calibration line and full set of QCs. Blank samples of each of the matrices were extracted using the optimised QuEChERS method to determine selectivity by ensuring the SIM/SRM scans for the internal standard are free of signal to ensure accurate quantitation can be carried out and screen for evidence of other pollutants. Solvent blanks were also run in between each sample type to ensure there was no carryover from the extracted matrices. A three-pronged approach to identification was adopted to determine the presence of target compounds within these samples, using the known retention times, the $m / z$ of precursor and any known product ions for each compound.

\subsection{1: Treated Sludgecake}

Two samples of sludgecake were collected from a wastewater treatment plant in South Wales at different times of year; a summer and a winter sample. These were chosen to preliminarily establish if the compounds of interest were detected at amounts influenced by perceived usage, i.e. the sample collected in summer contained higher quantities of pharmaceuticals such as antihistamines due to increased use, whereas the sample collected in winter contained more painkillers and NSAIDS from a greater use of cold and flu medication. The samples were analysed following extraction using the optimised preparation method, and searched for 
the target compounds then compared between sample types for any difference between the content and/or quantities of the compounds found.

The lipophilicity (i.e. $\log \mathrm{P}$ ) of the pharmaceuticals chosen suggest that the majority are likely to adsorb to sludge [2], with values of around 3 and above (see Appendix 2.1). Low $\log P$ values usually indicate that compounds are more likely to be hydrophilic, and therefore be present within aqueous samples. However, citalopram, carbamazepine and erythromycin have $\log \mathrm{P}$ values of $2.51,2.67$ and 2.83 respectively, indicating that the distribution of these compounds could be less distinct. Based upon the data provided by Berthod et al. [3] these lipophilicity constants could suggest a potential distribution between sludge, and the corresponding aqueous sample. Similarly for the biocides, the $\log \mathrm{P}$ values vary ranging from 1.69 for BAC-C12 to 4.28 for BAC-C18, increasing with the addition of carbon atoms. For the aliphatic analogues DDMA and HDTMA, both have a $\operatorname{low} \log \mathrm{P}$ of 2.51 and 2.40 respectively, however previous in-house data has shown that these biocides do adsorb to sludgecake.

Blank extracted samples were used to determine selectivity of the internal standards to ensure accurate quantitation can be carried out. Unfortunately, selectivity could not be achieved for acetaminophen-(methyl)- $\mathrm{d}_{3}(\mathrm{~m} / \mathrm{z}, 155)$ and talopram $(\mathrm{m} / \mathrm{z} 296)$ within the winter sample with background response observed at the same retention time as the standard, with the former possibly attributable to polar interferences near the solvent front, therefore the quantitation of acetaminophen, diphenhydramine, citalopram, fluoxetine and diclofenac was not able to be accurately assessed using these ISs. Similarly to the winter sample, the summer sample also showed limited selectivity for the selection of ISs with a background response for acetaminophen-(methyl) $-\mathrm{d}_{3}(\mathrm{~m} / \mathrm{z}, 155)$ and pronethalol $(\mathrm{m} / \mathrm{z}, 230)$. Given these observations the use of an alternative IS was investigated to ensure some quantitative measurement could be achieved.

\subsubsection{1: Evaluation of Alternative IS}

The calibration parameters were tested with alternate internal standards available within the relevant samples, i.e. using pronethalol for quantifying diphenhydramine, citalopram, fluoxetine and diclofenac, and talopram for propranolol, using the ZQ4000. The calibration data showed good linearity for each compound, with $\mathrm{R}^{2}$ values equal to 0.99 . The accuracy and precision were also tested using QC samples at multiple concentrations (i.e. 15, 25, 100, $350 \mathrm{ng} / \mathrm{mL}$ ) to assess the robustness of the method. Good precision and accuracy for each of 
the five pharmaceuticals tested, with results $<9 \%$ and $\leq 11 \%$ RSD respectively, confirming the usability of the alternative ISs for quantifying the relevant compounds within the sludgecake samples. The IDL values observed using these alternative ISs were equivalent to those determined in Chapter 3, with determined values $<2 \mathrm{ng} / \mathrm{mL}$.

Table 6.1: Summary of the $1 / x$ weighted quantitative data including linearity $\left(\mathrm{R}^{2}\right)$, instrument detection limit (IDL), mean percentage accuracy and precision of quality control sample (QC) concentration for a selection of pharmaceuticals using alternate internal standards (IS).

\begin{tabular}{|c|c|c|c|c|c|c|c|c|c|c|c|}
\hline \multirow{3}{*}{ Compound } & \multirow{3}{*}{$\begin{array}{l}\text { Internal } \\
\text { Standard } \\
\text { (IS) }\end{array}$} & \multirow{3}{*}{$\begin{array}{c}\text { Linearity } \\
\left(\mathbf{R}^{2}\right)\end{array}$} & \multirow{3}{*}{$\begin{array}{c}\text { IDL } \\
(\mathbf{n g} / \mathbf{m L})\end{array}$} & \multicolumn{8}{|c|}{ QCs } \\
\hline & & & & \multicolumn{4}{|c|}{ Mean Accuracy (\%) } & \multicolumn{4}{|c|}{ Mean Precision (\%) } \\
\hline & & & & QC1 & QC2 & QC3 & QC4 & QC1 & QC2 & QC3 & QC4 \\
\hline Propranolol & Talopram & 0.9993 & $0.20 \pm 0.1$ & 2.07 & 4.13 & 3.38 & 3.11 & 2.19 & 3.15 & 1.70 & 1.36 \\
\hline Diphenhydramine & Pronethalol & 0.9990 & $1.26 \pm 0.4$ & 0.63 & 0.90 & -2.28 & -1.37 & 1.74 & 2.39 & 2.10 & 1.86 \\
\hline Citalopram & Pronethalol & 0.9976 & $0.34 \pm 0.1$ & 11.92 & 11.90 & 4.26 & -3.96 & 1.62 & 1.25 & 1.07 & 2.21 \\
\hline Fluoxetine & Pronethalol & 0.9984 & $0.49 \pm 0.2$ & -2.74 & -4.63 & -5.54 & -1.09 & 0.43 & 2.86 & 4.60 & 3.06 \\
\hline Diclofenac & Pronethalol & 0.9981 & $1.61 \pm 0.5$ & -3.06 & 0.50 & -1.75 & 0.68 & 6.51 & 8.79 & 1.85 & 2.68 \\
\hline
\end{tabular}

\subsubsection{2: Winter Sample}

Of the ten pharmaceuticals in the suite, six were observed by both techniques (see Table 6.2). To confirm the presence of a precursor ion, the signal to noise ratio of the extracted ion chromatogram was calculated to establish if the signal observed was above a ratio of 3:1 (i.e. the IDL) [4]. Of the six pharmaceuticals detected; propranolol, diphenhydramine, citalopram, carbamazepine, loratadine and diclofenac, two compounds were quantifiable using the alternate IS calibration and these results confirm previous studies of pharmaceuticals within wastewater sludge, with concentrations of citalopram being reported between $60-300 \mathrm{ng} / \mathrm{g}$ [5,6,7], with reports of concentrations determined as high as $1000 \mathrm{ng} / \mathrm{g}$, within Europe [5]. Fluoxetine was also detected within the ZQ4000 data, however as only precursor ion data was observed, identification and determined concentration are tentative without further, confirmatory data. Although the concentration of fluoxetine is an estimation, the measurement is slightly higher than concentration reported within the literature with a previous study reporting approximately $200 \mathrm{ng} / \mathrm{g}$ within treated sludge [5]. 
Table 6.2: A summary of the identification factors used for the qualitative screen in the sample of sludgecake collected in winter. The retention time observed were the same over both platforms. *Fluoxetine and ibuprofen were detected within the ZQ4000 data only. **The main ion seen for diclofenac within the product ion scan on the LCQ was the precursor ion at $m / z$ 296, rather than the product ion at $m / z 277$.

\begin{tabular}{|l|c|c|c|c|}
\hline \multicolumn{1}{|c|}{ Compound } & $\begin{array}{c}\text { Retention } \\
\text { Time } \\
\text { (minutes) }\end{array}$ & $\begin{array}{c}\text { Signal: } \\
\text { Noise } \\
(\mathbf{S} / \mathbf{N})\end{array}$ & $\begin{array}{c}\text { Present in } \\
\text { both } \\
\text { techniques? }\end{array}$ & $\begin{array}{c}\text { Concentration } \\
(\boldsymbol{\mu g} / \mathbf{k g})\end{array}$ \\
\hline Propranolol & 15.8 & 7 & $\checkmark$ & - \\
\hline Diphenhydramine & 16.6 & 7 & $\checkmark$ & - \\
\hline Citalopram & 16.9 & 29 & $\checkmark$ & $995.3 \pm 126.7$ \\
\hline Carbamazepine & 18.1 & 3 & $\checkmark$ & - \\
\hline Fluoxetine $*$ & 18.7 & 10 & $\mathbf{x}$ & $319.5 \pm 43.6$ \\
\hline Loratadine & 19.2 & 3 & $\checkmark$ & - \\
\hline Diclofenac $* *$ & 24.4 & 3 & $\checkmark$ & - \\
\hline Ibuprofen $*$ & 23.8 & 5 & $\mathbf{x}$ & \\
\hline
\end{tabular}

Due to the high abundance of the biocides within sludgecake, a 1:400 dilution of the sludgecake extract was required before analysis on the LCQ (as described for the 1:400 soil extraction experiment in Section 5.2.2.1) with signal saturation also observed with ZQ4000 analysis as expected with its higher sensitivity (see Appendix 6.1). All six of the biocides were confirmed within the sludge sample, having a signal to noise greater than 3 . Of these, BAC-C12, BAC-C14, BAC-C18, HDTMA and DDMA appeared most abundant with concentrations exceeding beyond the recommendation of CIP II in milligram amounts.

Table 6.3: A summary of the identification factors used for the qualitative screen in the 1:400 dilution of the winter sample of sludgecake. The retention time observed matched those recorded for the standard sample. All of the compounds have a $\mathrm{S} / \mathrm{N}$ greater than 3 and therefore can be discerned from the background signal.

\begin{tabular}{|l|c|c|c|}
\hline Compound & $\begin{array}{c}\text { Retention } \\
\text { Time } \\
\text { (minutes) }\end{array}$ & $\begin{array}{c}\text { Signal: Noise } \\
\text { (S/N) }\end{array}$ & $\begin{array}{c}\text { Concentration } \\
\text { (mg/kg) }\end{array}$ \\
\hline BAC-C12 & 24.1 & 46 & $44.5 \pm 0.9$ \\
\hline BAC-C14 & 26.4 & 34 & $18.6 \pm 0.1$ \\
\hline BAC-C16 & 28.7 & 5 & - \\
\hline BAC-C18 & 30.9 & 178 & $5.5 \pm 0.9$ \\
\hline DDMA & 27.5 & 30 & $21.3 \pm 3.6$ \\
\hline HDTMA & 26.2 & 34 & $24.6 \pm 4.6$ \\
\hline
\end{tabular}

These concentrations are determined by legislation outlined in European Directive (2013/39/EU), stating the required limit of detection for emerging compounds, (including pharmaceuticals) detected in sludge samples should not exceed $0.1 \mu \mathrm{g} / \mathrm{kg}$. Importantly, these 
results are consistent with reports that concentrations of QACs between 10-50 mg/L are considered microbicidal [8], assuming that $1 \mathrm{~L}=1 \mathrm{~kg}$. Therefore, the use of this sludgecake as an agricultural fertiliser may be important in considering the impact of this pollutant, specifically in leading to an increased selective pressure towards antibiotic resistance. Given this potential link, this data suggests that QACs should be viewed as an emerging compound of interest, and the remit of CIP to be extended beyond pharmaceuticals.

\subsubsection{3: Summer Sample}

Similarly to the winter sample, three of the pharmaceuticals were detected within the summer sample and confirmed using the product ion scans of the LCQ data (see Table 6.4). However, these compounds were observed at levels that were unable to be quantified, as the $\mathrm{S} / \mathrm{N}$ determined for each compound detected was less than 10 .

Although the precursor ion for diclofenac was observed at the correct retention time within the confirmatory screen, there was insufficient product ion signal for confirmation. This is not surprising given diclofenac provided the lowest $\mathrm{S} / \mathrm{N}$ precursor ion of those detected.

Table 6.4: A summary of the identification factors used for the qualitative screen in the sample of sludgecake collected in summer. The retention time of the standard was taken from the top calibration standard $(400 \mathrm{ng} / \mathrm{mL})$ analysed on the same day as the sludge sample.

\begin{tabular}{|l|c|c|c|c|}
\hline Compound & $\begin{array}{c}\text { Retention } \\
\text { Time } \\
(\text { minutes) }\end{array}$ & $\begin{array}{c}\text { Signal: } \\
\text { Noise } \\
(\mathbf{S} / \mathbf{N})\end{array}$ & $\begin{array}{c}\text { Present in } \\
\text { both } \\
\text { techniques? }\end{array}$ & $\begin{array}{c}\text { Concentration } \\
(\boldsymbol{\mu g} / \mathbf{k g})\end{array}$ \\
\hline Propranolol & 15.9 & 3 & $\checkmark$ & - \\
\hline Carbamazepine & 18.1 & 5 & $\checkmark$ & - \\
\hline Loratadine & 20.5 & 5 & $\checkmark$ & - \\
\hline Diclofenac $*$ & 24.4 & 3 & $\checkmark$ & - \\
\hline Ibuprofen** & 23.7 & 6 & & \\
\hline
\end{tabular}

While the difference between these samples did not meet the assumption that the contents would reflect the season as more antihistamine medications, such as diphenhydramine and loratadine, were detected in higher amounts within the winter samples, it does prove that the method works for multiple samples collected at different time points throughout the year.

As with the winter sludgecake, all six of the biocides of interest were detected within the summer sludgecake samples with the concentration range of $2.7-70.4 \mathrm{mg} / \mathrm{kg}$. 
Table 6.5: A summary of the identification factors used for the qualitative screen in the 1:400 dilution of the summer sample of sludgecake. The retention time observed matched those recorded for the standard sample. All of the compounds have a $\mathrm{S} / \mathrm{N}$ greater than 3 and therefore can be discerned from the background signal.

\begin{tabular}{|l|c|c|c|}
\hline Compound & $\begin{array}{c}\text { Retention } \\
\text { Time } \\
\text { (minutes) }\end{array}$ & $\begin{array}{c}\text { Signal: Noise } \\
\text { (S/N) }\end{array}$ & $\begin{array}{c}\text { Concentration } \\
(\mathbf{m g} / \mathbf{k g})\end{array}$ \\
\hline BAC-C12 & 24.1 & 295 & $70.4 \pm 25.9$ \\
\hline BAC-C14 & 26.4 & 162 & $32.1 \pm 12.1$ \\
\hline BAC-C16 & 28.7 & 30 & $2.7 \pm 0.4$ \\
\hline BAC-C18 & 31.0 & 13 & $2.8 \pm 0.5$ \\
\hline DDMA & 27.5 & 183 & $26.8 \pm 6.4$ \\
\hline HDTMA & 26.3 & 109 & $41.4 \pm 14.7$ \\
\hline
\end{tabular}

Again, high concentrations of biocides were observed at milligram level within the summer sludgecake sample (see Table 6.5), however these were significantly higher than those recorded within the winter sample, with a mean percentage change of $47 \%$, except for BACC18 (see Figure 6.1), suggesting the usage of disinfectants change between different seasons potentially due to the use of BACs as an algaecide for swimming pools [9].

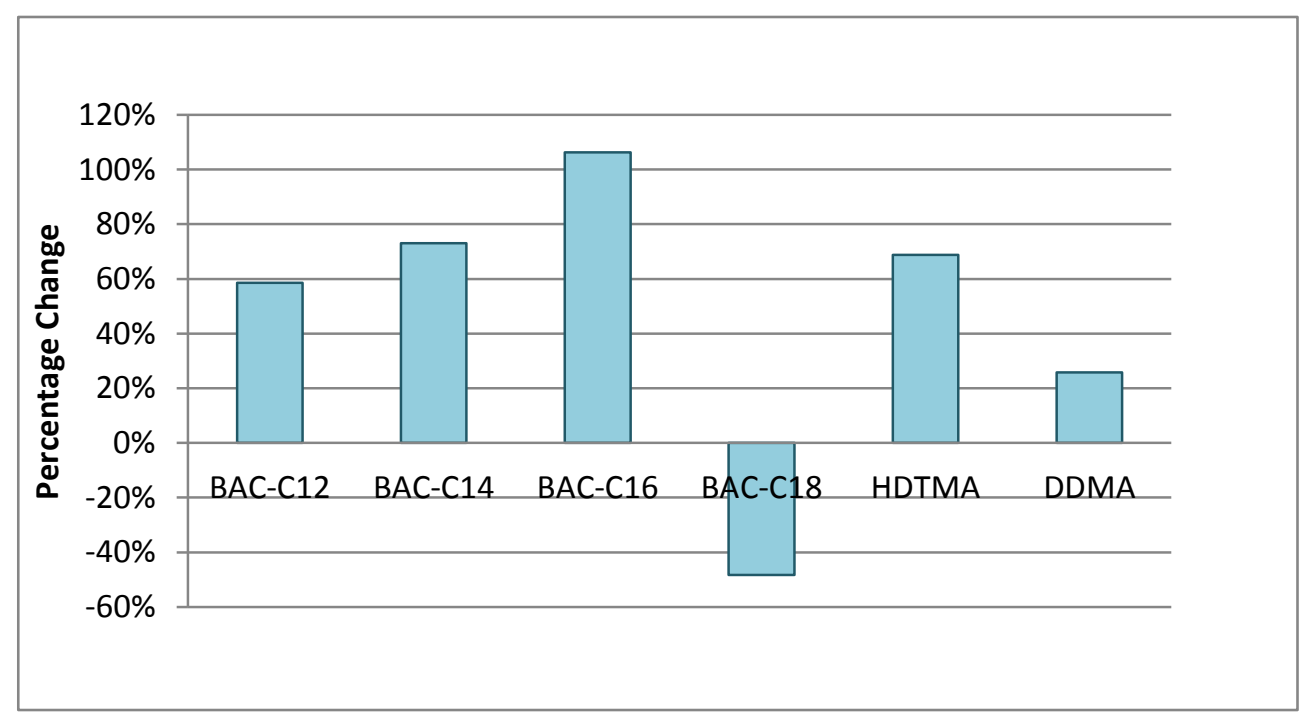

Figure 6.1: A bar chart showing the percentage change in calculated concentration of the biocides between the samples of sludgecake collected during the winter and summer.

\subsection{2: Treated Effluent}

To meet the legislation outlined within the Water Framework Directive (2000/60/EC), detailing the requirement that all UK watercourses should be monitored and have a good status [10], the QuEChERS method was applied to a complimentary sample of treated 
effluent collected from the same wastewater treatment plant as the sludgecake, to determine what CIP compounds could be observed within a liquid wastewater fraction. Again a background response was measured at the transition and retention time for acetaminophen(methyl)- $\mathrm{d}_{3}$, therefore it was not used for quantitation.

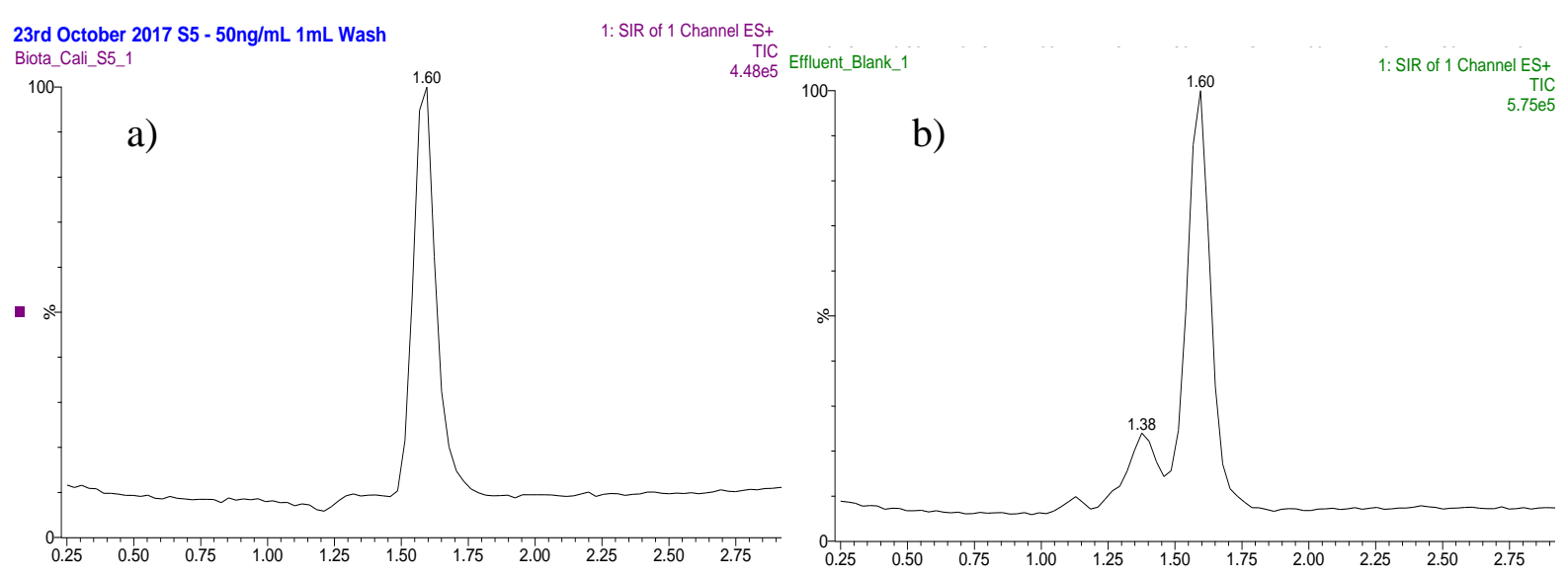

Figure 6.2: The total ion chromatograms (TIC) for a) a standard of acetaminophen-(methyl)- $\mathrm{d}_{3}$ compared to b) an extracted blank sample of treated effluent showing the background signal recorded at the SIM scan for acetaminophen-(methyl)- $\mathrm{d}_{3}$. As there is no selectivity quantitation of acetaminophen will not be possible.

Based on the CIP selection criteria of lipophilic substances $(\log \mathrm{P}>3)$ it was anticipated that these would not be observed within the effluent sample as they would be more likely to adsorb to sludge. Precursor ions indicative of propranolol, citalopram and carbamazepine were observed within this sample; these compounds have $\log \mathrm{P}$ values $\leq 3$, indicating slight lipophilicity [11], meaning that while they are observed in sludgecake, they are also likely to be seen within the water fraction. However, despite precursor ions being observed within the SIM scan on the ZQ4000, the signal intensity of these "peaks" was low $\left(\times 10^{4}\right)$ and had a S/N $<3$, therefore any positive detection/identification would be inaccurate.

The data has shown that biocides adsorb to sludgecake and are detected in high abundance however, the behaviour of biocides in other wastewater fractions, such as effluent, is more complicated to predict. The $\log \mathrm{P}$ values indicate that they are hydrophilic and should be detected within effluent, potentially due to decrease in concentration of "free" surfactant within the liquid due to the formation of micelles once the surfactant concentrations are above the critical micelle concentration (CMC) [12]. This behaviour is indicated by the data, as the suite of biocides were observed at significantly lower concentrations (see Figure 6.3) with BAC-C12 and BAC-C16 quantifiable above the IDL at 1.1 and $1.6 \mathrm{ng} / \mathrm{mL}$ respectively. These values are slightly lower than those reported in the literature, with values of 
approximately $0.062 \mathrm{mg} / \mathrm{L}$ (i.e. $62 \mathrm{ng} / \mathrm{mL}$ ) quantified in sewage effluent in Croatia [13], however, this could be due to usage, including its possible use as a dewatering agent during the wastewater process within this Member State.

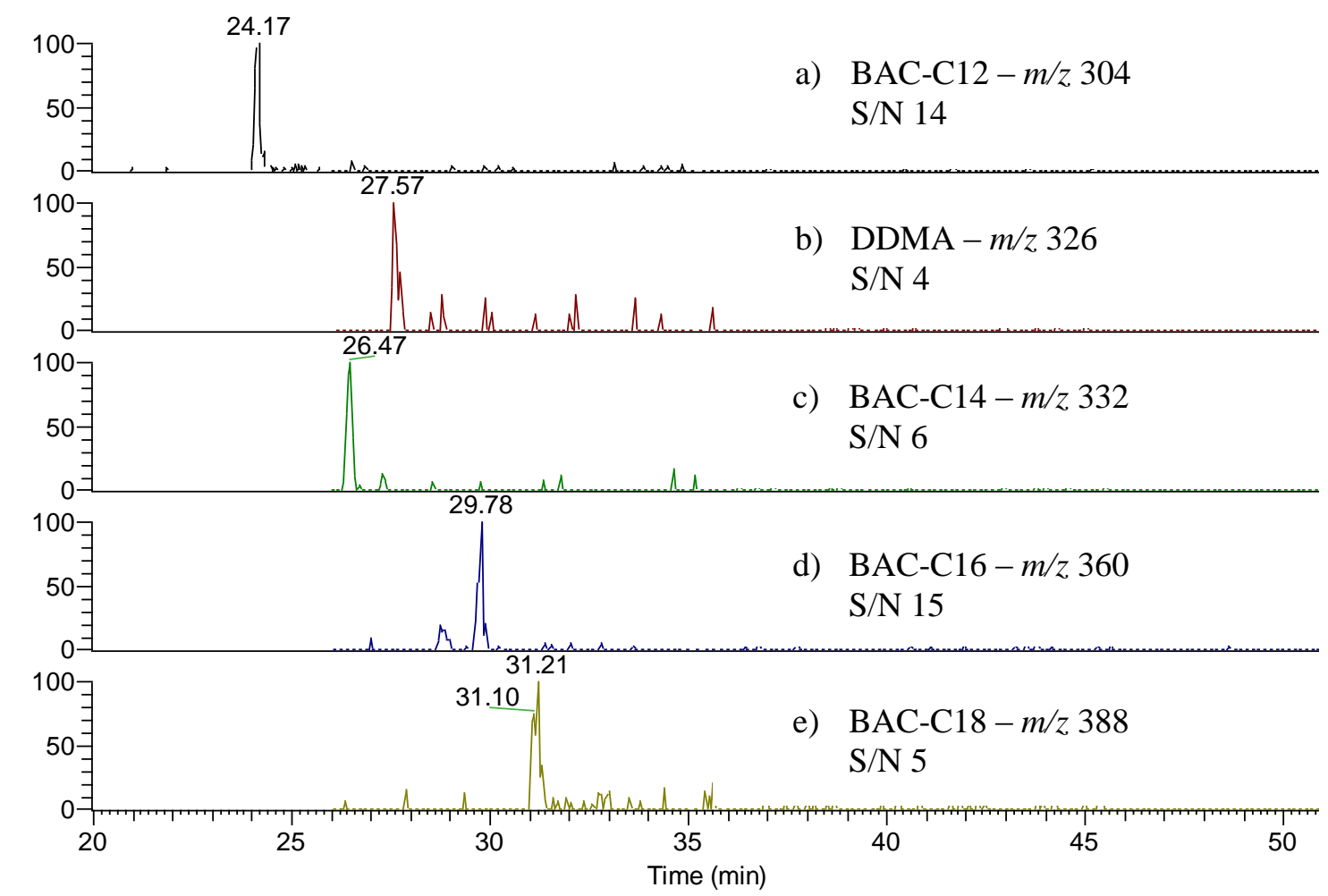

Figure 6.3: Extracted ion chromatograms showing the biocides detected within effluent a) BAC-C12, b) DDMA, c) BAC-C14, d) BAC-C16 and e) BAC-C18 with the signal-to-noise for each peak.

\subsection{3: Biota}

A sample of locally sourced mussel tissue was homogenised and extracted using the optimised QuEChERS method to determine whether the pharmaceuticals and biocides accumulate similarly to sludgecake due to a comparative lipid content $[14,15]$. A "wet" sample was analysed alongside a lyophilised aliquot, in keeping with the sample pretreatment of the sludgecake. The total ion chromatogram recorded for both the wet and lyophilised aliquots looked similar to sludgecake, with lyophilised biota showing a slightly higher background signal, possibly due to the concentration effect of freeze-drying, see Figure 6.4. 


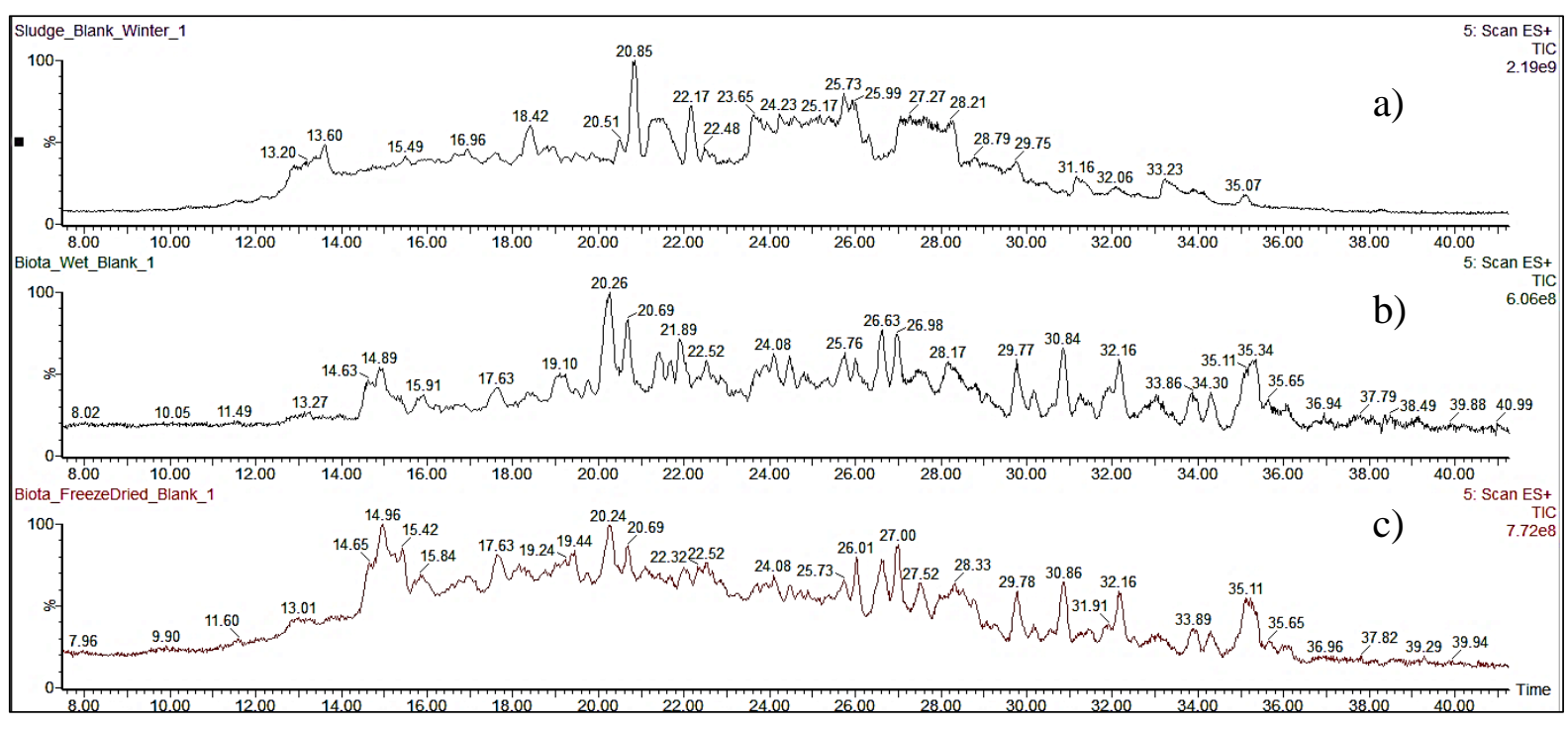

Figure 6.4: The total ion chromatograms (TIC) for a) an extracted blank sample of treated sludgecake b) an extracted blank sample of wet homogenised biota and c) an extracted blank sample of lyophilised homogenised biota, which show a higher background signal for the sludgecake and biota samples that were lyophilised.

Once again, in both samples, there is limited selectivity at the transition for acetaminophen(methyl)- $\mathrm{d}_{3}$, with a background response being recorded at the retention time. As with all the matrices examined, there is interference with the solvent front and therefore reliable quantitation of acetaminophen was not possible.

\subsubsection{1: Wet Biota}

Three pharmaceuticals were detected within this sample; propranolol, diclofenac and ibuprofen, with a $\mathrm{S} / \mathrm{N}$ of 4,5 and 9 , respectively, therefore quantitation of these peaks was unachievable. The majority of the SIM scans for the pharmaceuticals did not show a discernible signal from the background, however, the extracted ion chromatograms of the full mass scan showed a strong signal indicative of fluoxetine (S/N 177), loratadine (S/N 8) and another peak for diclofenac (S/N 59) at differing retention times than typically observed. These peaks were also observed within the LCQ data, however only the precursor ion was seen for fluoxetine and loratadine, while the product ion for diclofenac was observed. 


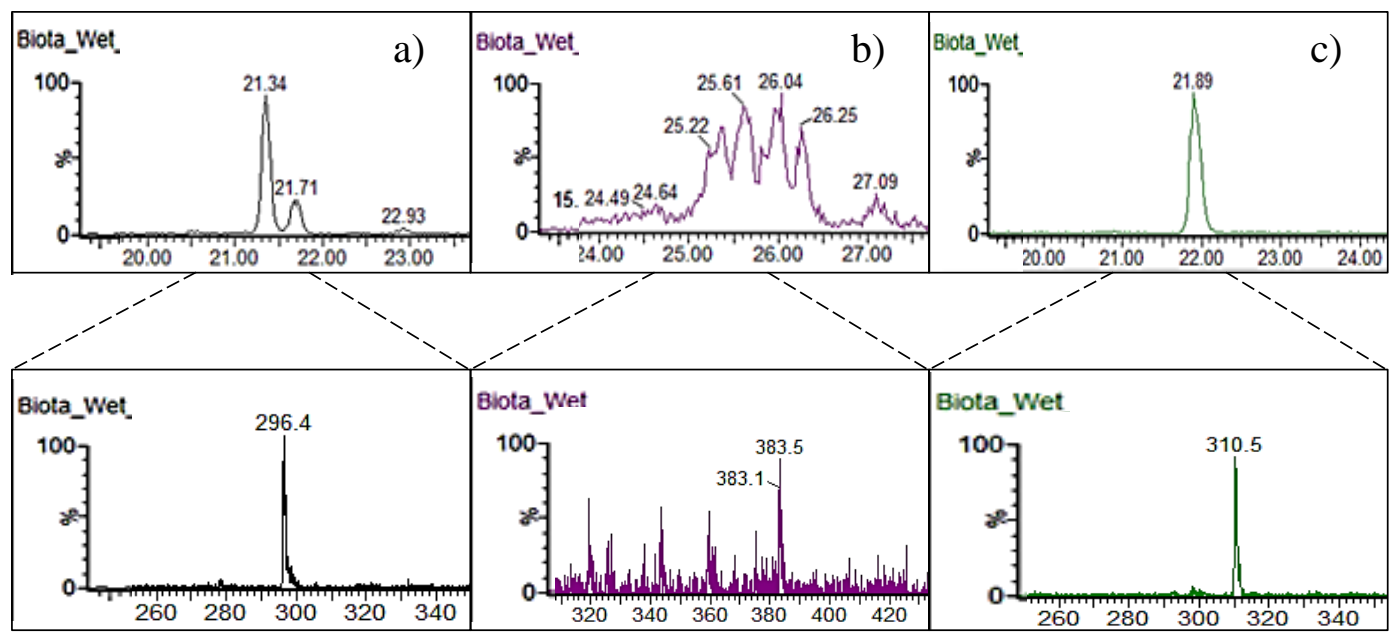

Figure 6.5: The extracted ion chromatograms showing the later elution times for a) diclofenac $(\mathrm{m} / \mathrm{z} 296) \mathrm{b})$ loratadine $(\mathrm{m} / \mathrm{z}, 383) \mathrm{c})$ fluoxetine $(\mathrm{m} / \mathrm{z} 310)$ and the corresponding expanded spectra showing the precursor ions for each of the compounds.

In addition to pharmaceuticals, there was significant evidence suggesting the target biocides were present, specifically, BAC-C12, BAC-C14, BAC-C18 and DDMA. This is important as it provides evidence to suggest that these are either mobilised from farmland through agricultural run-off or are present within the "clean" water fraction that is dispensed into the water course. These were observed at lower amounts than sludgecake, with determined concentrations of BAC-C12, BAC-C18 and DDMA found to be 7.4, 6.7 and $8.1 \mu \mathrm{g} / \mathrm{kg}$, respectively, perhaps suggesting that direct disposal into the water habitat for these animals is less likely and other, more indirect routes described above are more likely to be the route of exposure here.

\subsubsection{2: Lyophilised Biota}

Lyophilised (freeze-dried) biota was analysed to determine whether the removal of water from the sample had affected the concentration of the compounds of interest observed. Similar to the wet biota sample, three pharmaceuticals were detected by both techniques; carbamazepine, loratadine and ibuprofen, and additionally, diclofenac was detected within the SIM data only. Extracted ion chromatograms of the full mass scan showed a signal indicative of citalopram ( $/ \mathrm{N}$ 6) and fluoxetine (S/N 16), with additional peaks indicative of loratadine (S/N 7) and diclofenac (S/N 14) at differing retention time than typically observed. The majority of the literature regarding pollutants in biota are concerning the concentration of pharmaceuticals within mussel tissue, and show that carbamazepine is the most commonly detected within different species of biota. A UK based study has also shown this within 
Gammarus Pulex from the Thames, London, where a concentration of $6 \mathrm{ng} / \mathrm{g}$ of carbamazepine was recorded [16], and a study conducted on multiple classes of biota in Spain reported a concentration of $1.3 \mathrm{ng} / \mathrm{g}$ of carbamazepine. Other pharmaceuticals include citalopram, with a reported concentration of $1.9 \mathrm{ng} / \mathrm{g}$ within Crassostrea gigas (Pacific oyster) [17]. Positively, although the species of biota differs to this study, both carbamazepine and citalopram were detected within the biota samples tested, albeit at a concentration below the IQL.

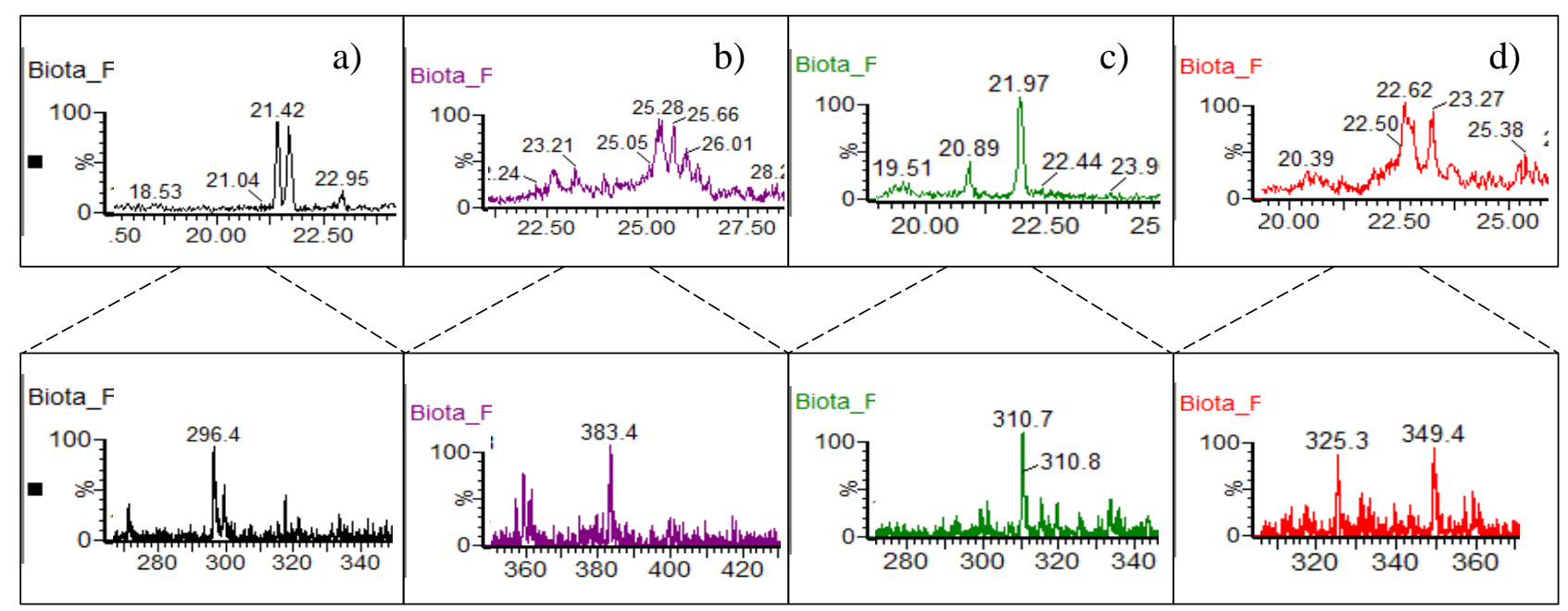

Figure 6.6: The extracted ion chromatograms showing the later elution times for a) diclofenac $(\mathrm{m} / \mathrm{z}$ 296) b) loratadine $(\mathrm{m} / \mathrm{z} 383) \mathrm{c})$ fluoxetine $(\mathrm{m} / \mathrm{z} 310) \mathrm{d})$ citalopram $(\mathrm{m} / \mathrm{z} 325)$ and the corresponding expanded spectra showing the precursor ions for each of the compounds.

Similar to the wet samples, BAC-C12, BAC-C18 and DDMA were also detected at lower concentrations, these made the precursor ions difficult to distinguish from the complex matrix interferences. Only BAC-C12 had a distinct signal within the SRM, with $\mathrm{S} / \mathrm{N}>10$, and a measured concentration of $5.5 \mu \mathrm{g} / \mathrm{kg}$, slightly lower than the concentration seen in the wet sample, which could be due to "free" BAC binding to certain lipid classes [18] within the biota sample (i.e. phospholipids), potentially as a result of the reduction of liquid during lyophilisation, however further analysis would need to be conducted for confirmation.

\subsubsection{3: Manual Integration of Later Eluting Peaks}

Both samples of biota showed peaks with precursor ions consistent with fluoxetine, loratadine, and diclofenac, and a peak consistent with citalopram was detected within the lyophilised sample. These were integrated manually within the extracted ion chromatogram for quantitation as they were not captured by the automated processing method. To ensure 
consistency each peak of interest was automatically integrated using the chromatogram window within MassLynx and then manually divided by the automated peak area of the corresponding internal standard to generate the RRF, used to determine concentration. The calculated values are much higher than those reported within the literature, and also those in the SIM scan.

Table 6.6: A summary of the concentrations of pharmaceuticals detected and quantified using manual integration of the extracted ion chromatogram within the two homogenised biota samples tested.

\begin{tabular}{|c|c|c|}
\cline { 2 - 3 } \multicolumn{1}{c|}{} & \multicolumn{2}{c|}{$\begin{array}{c}\text { Concentration in Biota } \\
(\boldsymbol{\mu g} / \mathbf{k g})\end{array}$} \\
\hline Compound & Wet & Lyophilised \\
\hline Fluoxetine & 499.5 & 77.4 \\
\hline Diclofenac & 4511.1 & 894.8 \\
\hline
\end{tabular}

As these values were calculated from peaks with a later retention time than expected, further data is needed to support the identification of these compounds, so these calculated concentrations can only be used as an estimation of quantities of contaminants within this matrix.

\section{2: Qualitative Screen using Accurate Mass}

To support the quantitative analysis performed on the ion trap and quadrupole platforms, a qualitative screen was carried out using an LTQ Orbitrap mass spectrometer using data dependant acquisition (DDA). Extracted matrix blanks were analysed to obtain confirm selectively. Similarly to the quantitative analysis, a three-pronged approach was used to identify compounds of interest; the retention time, precursor (full mass scan) and product ion (DDA) data based on known standards targeted within this project. Using the high mass resolution capability of this platform, elemental formula were proposed for both precursor and fragment ion species, assisting in identification along with any observed isotope patterns.

\subsection{1: Treated Sludgecake}

The samples were first searched to determine whether the target compounds were detected, corroborating the findings of the quantitative analysis. Of the target pharmaceuticals and biocides, five pharmaceuticals and six biocides were identified using the parameters described above, with assigned elemental formulas matching with an error of <1ppm. In addition to the identification of the target compounds, the data was represented and studied 
using the base peak chromatogram to help identify the most abundant species recorded. Within the two samples of sludgecake (one collected in winter and one collected in summer) there were 10 peaks of interest common to both samples at similar retention times, over a mass range of $m / z$ 200-700. The compounds with lower $m / z$ (between $m / z$ 200-370) eluted towards the end of the chromatographic gradient, between 22-27 minutes, similar to the target QACs (see Appendix 6.2). These compounds did not appear to show distinctive isotope patterns indicative of halogenated species but a simple carbon isotope. Using the elemental formula search these unidentified compounds showed similarities to the QACs, with most consisting of carbon, hydrogen and nitrogen only, indicative of ammonium biocides, with the exception of $\mathrm{m} / \mathrm{z} 211.0867$ and 258.2796; these were predicted to contain an oxygen atom, supported by the product ion data with losses consistent with $\mathrm{H}_{2} \mathrm{O}$ (18 Da).

Table 6.7: A summary of the ions common to the winter and summer sludgecake samples, showing the retention time $(\mathrm{RT})$, mass-to-charge $(\mathrm{m} / \mathrm{z})$ and possible chemical formulas generated from Xcalibur 3.0 with the associated error of $<1 \mathrm{ppm}$, providing confidence in the identification.

\begin{tabular}{|c|c|c|c|c|c|c|}
\hline RT & $\boldsymbol{m} / \boldsymbol{z}$ & $\begin{array}{c}\text { Possible } \\
\text { Formula }\end{array}$ & $\begin{array}{c}\text { Error } \\
(\mathbf{p p m})\end{array}$ & $\begin{array}{c}\text { Product } \\
\text { Ion }\end{array}$ & $\begin{array}{c}\text { Possible } \\
\text { Formula }\end{array}$ & $\begin{array}{c}\text { Error } \\
(\mathbf{p p m})\end{array}$ \\
\hline 22.24 & 211.0867 & $\mathrm{C}_{13} \mathrm{H}_{11} \mathrm{~N}_{2} \mathrm{O}$ & 0.25 & $\begin{array}{c}193.0765 \\
169.0762\end{array}$ & $\begin{array}{c}\mathrm{C}_{13} \mathrm{H}_{9} \mathrm{~N}_{2} \\
\mathrm{C}_{11} \mathrm{H}_{9} \mathrm{~N}_{2}\end{array}$ & $\begin{array}{c}0.45 \\
0.16\end{array}$ \\
\hline 23.93 & 258.2796 & $\mathrm{C}_{16} \mathrm{H}_{36} \mathrm{NO}$ & 0.27 & 214.2531 & $\mathrm{C}_{14} \mathrm{H}_{32} \mathrm{~N}$ & 0.18 \\
\hline 24.56 & 200.2372 & $\mathrm{C}_{13} \mathrm{H}_{30} \mathrm{~N}$ & -0.09 & - & - & - \\
\hline 25.80 & 228.2684 & $\mathrm{C}_{15} \mathrm{H}_{34} \mathrm{~N}$ & -0.09 & - & - & - \\
\hline 25.80 & 270.3155 & $\mathrm{C}_{18} \mathrm{H}_{40} \mathrm{~N}$ & 0.42 & 158.1907 & $\mathrm{C}_{10} \mathrm{H}_{24} \mathrm{~N}$ & -0.26 \\
\hline 33.43 & 368.4256 & $\mathrm{C}_{25} \mathrm{H}_{54} \mathrm{~N}$ & -0.05 & - & - & - \\
\hline
\end{tabular}

The four peaks of interest with higher $\mathrm{m} / \mathrm{z}$ at 520.3323, 608.3846, 652.4119 and 696.4381, eluted earlier in the chromatogram, around 14 minutes. Again, these compounds only showed an isotope pattern consistent with ${ }^{13} \mathrm{C}$, and product ion data showed a loss of 17 or 18 , which is typically related to the loss of $\mathrm{NH}_{3}$ or $\mathrm{H}_{2} \mathrm{O}$, respectively. A study of PEG 400 and its oligomers by Bhaskar et al. [19] using ESI showed similarities in the observed ions. All four ions of interest were present within the literature and identified as polyethylene glycol 400 (PEG 400) oligomers that had formed ammonium adducts. This supports the product ion data observed within the sludgecake samples, and as PEG 400 is commonly used as a pharmaceutical excipient [20] there is reason to support the presence of these compounds within sludge however, further work with standards would need to be carried out to positively identify these peaks. 


\subsection{2: Effluent}

The quantitative method for the effluent sample showed there was limited compound recovery for the pharmaceuticals, due to the lipophilicity of the analytes, indicating their proclivity to adsorb to sludge. However, similarly to the quantitative analysis, ions indicative of the full suite of biocides were detected within the qualitative screen, with BAC-C12 detected in highest abundance. Product ion data was recorded for BAC-C12 only, with identification of the other biocides achieved using the precursor $\mathrm{m} / \mathrm{z}$. This further supports the literature that QACs are present within aqueous samples, albeit at lower amounts due to potential micelle form reducing the concentration of "free" surfactant [12].

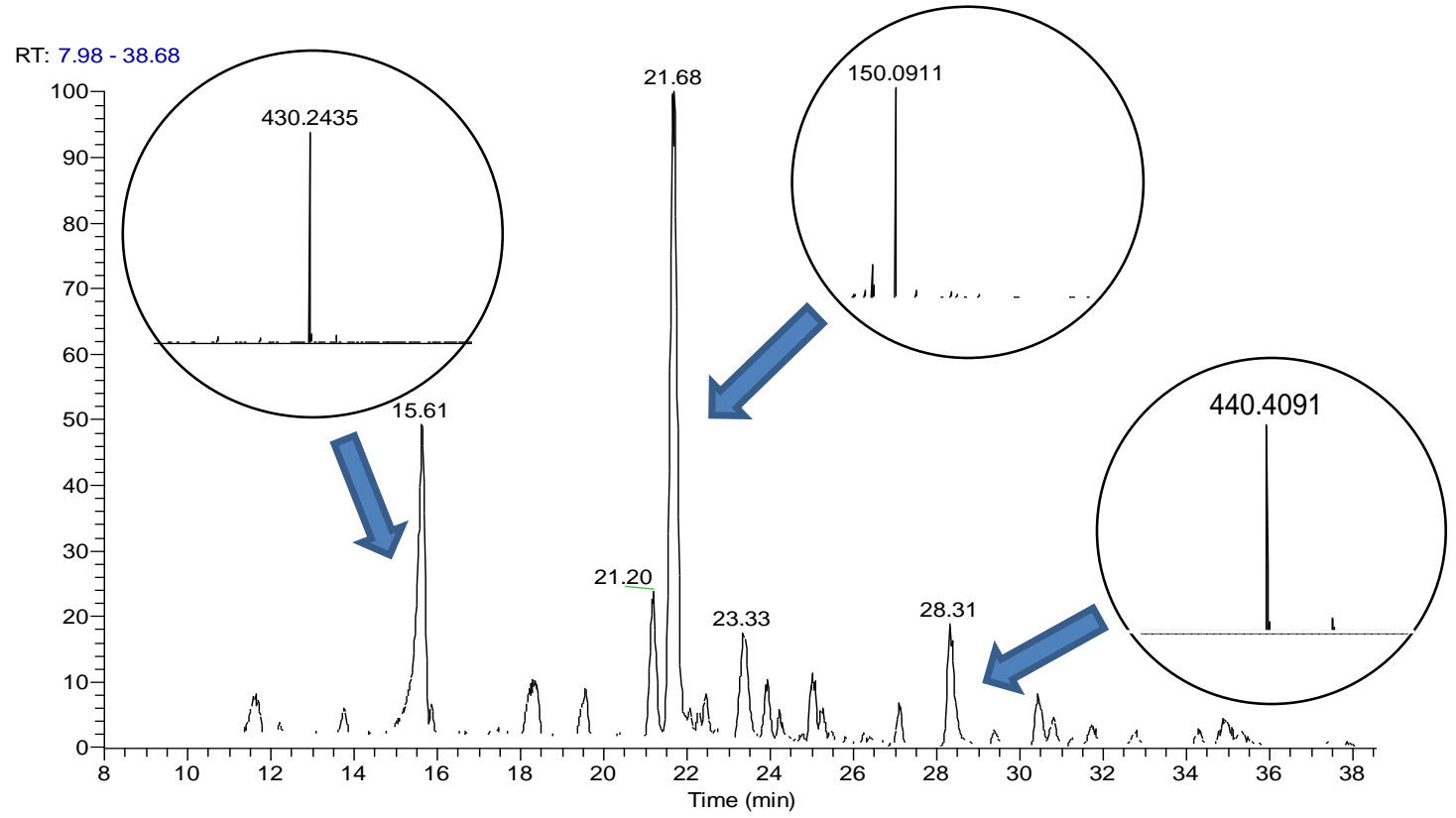

Figure 6.7: The chromatogram showing the peaks of interest for treated effluent, with the corresponding mass spectra of $m / z, 430.2435,150.0911$ and 440.4091 .

In addition to the identification of the target compounds, a series of peaks within the base peak chromatogram eluting between 15-30 minutes were investigated. The 3 peaks of interest had a similar $m / z$ range of $400-450$, with the exception of the base peak at 21 minutes, which was $m / z$ 150. Unfortunately the DDA did not manage to record product ion data so potential identification is difficult. From previous studies in the literature a peak at $\mathrm{m} / \mathrm{z} 150.0911$ in wastewater effluent has been observed, attributable to methamphetamine $\left(\mathrm{C}_{10} \mathrm{H}_{15} \mathrm{~N}\right)$ [21], however the determined accurate mass does not reflect this elemental formula, therefore further analysis is needed to identify this compound. Similar issues were encountered with the remaining peaks of interest at $\mathrm{m} / \mathrm{z} 430.2435$ and 440.4091 ; each had corresponding 
product ion data and the elemental formulas generated were $\leq 1 \mathrm{ppm}$, however, definitive identification of these peaks is not possible without further analytical data.

Table 6.8: A summary of the ions identified within the effluent sample, showing the retention time (RT), massto-charge $(\mathrm{m} / \mathrm{z})$ and possible chemical formulas generated from Xcalibur 3.0 with the associated error of $\leq 1 \mathrm{ppm}$, providing confidence in the identification.

\begin{tabular}{|c|c|c|c|c|c|c|}
\hline RT & $\boldsymbol{m} / \boldsymbol{z}$ & $\begin{array}{c}\text { Possible } \\
\text { Formula }\end{array}$ & $\begin{array}{c}\text { Error } \\
(\mathbf{p p m})\end{array}$ & $\begin{array}{c}\text { Product } \\
\text { Ion }\end{array}$ & $\begin{array}{c}\text { Possible } \\
\text { Formula }\end{array}$ & $\begin{array}{c}\text { Error } \\
(\mathbf{p p m})\end{array}$ \\
\hline 15.61 & 430.2430 & $\mathrm{C}_{21} \mathrm{H}_{36} \mathrm{O}_{8} \mathrm{~N}$ & -0.40 & 216.1232 & $\mathrm{C}_{10} \mathrm{H}_{18} \mathrm{O}_{4} \mathrm{~N}$ & 0.22 \\
& & $\mathrm{C}_{20} \mathrm{H}_{30} \mathrm{O}_{3} \mathrm{~N}_{8}$ & -0.41 & & $\mathrm{C}_{8} \mathrm{H}_{16} \mathrm{O}_{3} \mathrm{~N}_{4}$ & 1.56 \\
\hline 28.31 & 440.4091 & $\mathrm{C}_{25} \mathrm{H}_{52} \mathrm{O}_{2} \mathrm{~N}_{4}$ & 0.52 & 226.2166 & $\mathrm{C}_{12} \mathrm{H}_{26} \mathrm{~N}_{4}$ & 1.35 \\
\hline
\end{tabular}

\subsection{3: Biota}

Surprisingly, the detection of target analytes within the two biota samples was the same, indicating that the lyophilisation process had little effect on potential pollutants within the samples. Each sample had precursor ion data for 4 pharmaceuticals and 1 biocide; propranolol, fluoxetine, loratadine, diclofenac and BAC-C12, however only BAC-C12 could be positively identified due to the additional product ion data recorded. The precursor ion, $\mathrm{m} / \mathrm{z} 304.2999$ and product ion, $\mathrm{m} / \mathrm{z} 212.2375$ were observed at a corresponding retention time and formulas, $\mathrm{C}_{21} \mathrm{H}_{38} \mathrm{~N}$ and $\mathrm{C}_{14} \mathrm{H}_{30} \mathrm{~N}(<1 \mathrm{ppm})$.

As with the previous matrices, the base peak chromatograms for both biota samples were investigated. Despite the difference in preparation between the two samples (wet and lyophilised), there were 7 main peaks of interest common to both samples at similar retention times, at $\mathrm{m} / \mathrm{z} 430.2432,150.0909,482.3591,228.2320,254.2477,280.2639$ and 282.2791 (see Figure 6.8).

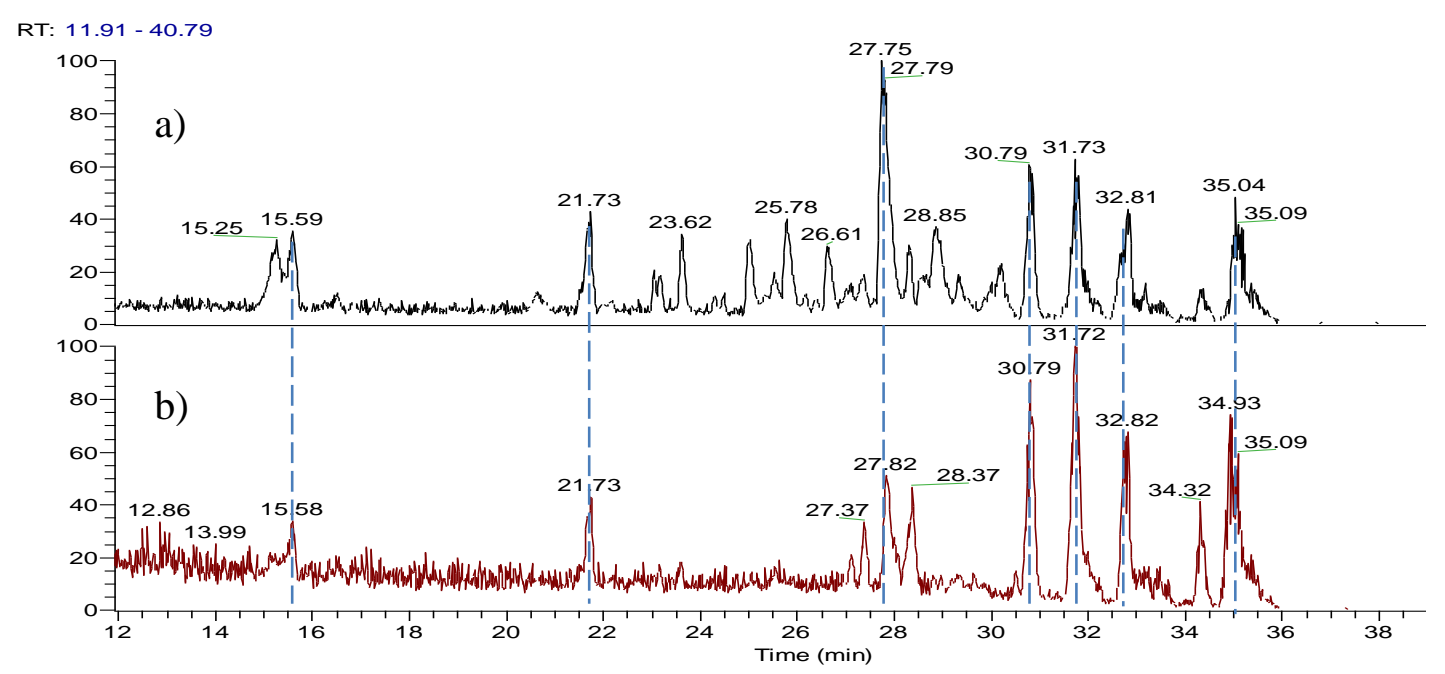

Figure 6.8: The chromatograms showing the common peaks of interest between a) wet and b) lyophilised biota. 
The first two peaks, $m / z 430.2432$ and 150.0909 are also observed with the treated effluent sample at similar retention times of 15 and 21 minutes, respectively. Isotope patterns were assessed and each compound showed an isotope consistent with the ${ }^{13} \mathrm{C}$ only, ruling out the presence of any chlorine or bromine atoms. Product ion data recorded for $m / z, 430.2432$ corresponds to a loss of $214 \mathrm{Da}$, leaving a product ion $\mathrm{m} / \mathrm{z} 216.1231$, with potential chemical formulas correlating to $\mathrm{C}_{21} \mathrm{H}_{36} \mathrm{O}_{8} \mathrm{~N}$ and $\mathrm{C}_{10} \mathrm{H}_{18} \mathrm{O}_{4} \mathrm{~N}$, respectively, consistent with the effluent sample. A product ion consistent with a loss of 17 was observed for $\mathrm{m} / z$ 150.0909, leaving $\mathrm{m} / \mathrm{z}$ 133.0881; however, the only formulas generated for these ions that are consistent with a loss of $\mathrm{NH}_{3}$ are $\mathrm{C}_{8} \mathrm{H}_{12} \mathrm{~N}_{3}$ and $\mathrm{C}_{8} \mathrm{H}_{9} \mathrm{~N}_{2}$ respectively, with an associated error of <12ppm, therefore without more complimentary analytical data, positive identification of these peaks is not possible. While there was no product ion data recorded for $m / z 228.2320,280.2639$ and 282.2791, the DDA for $m / z 482.3591$ shows an ion consistent with a loss of $\mathrm{H}_{2} \mathrm{O}(18 \mathrm{Da})$, indicating the presence of at least one oxygen atom and for $m / z 254.2477$ a product ion consistent with a loss of $\mathrm{NH}_{3}(17 \mathrm{Da})$ was observed at $m / z$ 237.2211, however, further analysis would be required to achieve a positive identification of these peaks.

Table 6.9: A summary of the ions common to the wet and lyophilised biota samples, showing the retention time (RT), mass-to-charge $(\mathrm{m} / \mathrm{z})$ and possible chemical formulas generated from Xcalibur 3.0 with the associated error.

\begin{tabular}{|c|c|c|c|c|c|c|}
\hline RT & $m / z$ & $\begin{array}{l}\text { Possible } \\
\text { Formula }\end{array}$ & $\begin{array}{l}\text { Error } \\
(\mathbf{p p m})\end{array}$ & $\begin{array}{l}\text { Product } \\
\text { Ion }\end{array}$ & $\begin{array}{l}\text { Possible } \\
\text { Formula }\end{array}$ & $\begin{array}{l}\text { Error } \\
\text { (ppm) }\end{array}$ \\
\hline 15.58 & 430.2432 & $\mathrm{C}_{21} \mathrm{H}_{36} \mathrm{O}_{8} \mathrm{~N}$ & -0.65 & 216.1231 & $\mathrm{C}_{10} \mathrm{H}_{18} \mathrm{O}_{4} \mathrm{~N}$ & 0.07 \\
\hline 21.73 & 150.0909 & $\mathrm{C}_{8} \mathrm{H}_{12} \mathrm{~N}_{3}$ & -11.7 & 133.0881 & $\mathrm{C}_{8} \mathrm{H}_{9} \mathrm{~N}_{2}$ & 12.20 \\
\hline 27.82 & 482.3591 & $\mathrm{C}_{27} \mathrm{H}_{44} \mathrm{ON}_{7}$ & -1.11 & 464.3503 & $\mathrm{C}_{27} \mathrm{H}_{42} \mathrm{~N}_{7}$ & 0.62 \\
\hline 30.79 & 228.2320 & $\begin{array}{l}\mathrm{C}_{14} \mathrm{H}_{30} \mathrm{ON} \\
\mathrm{C}_{12} \mathrm{H}_{28} \mathrm{~N}_{4}\end{array}$ & $\begin{array}{l}-0.18 \\
1.16 \\
\end{array}$ & - & - & - \\
\hline 31.72 & 254.2477 & $\mathrm{C}_{14} \mathrm{H}_{30} \mathrm{~N}_{4}$ & 1.23 & 237.2211 & $\mathrm{C}_{14} \mathrm{H}_{27} \mathrm{~N}_{3}$ & 1.20 \\
\hline 32.81 & 280.2639 & $\begin{array}{l}\mathrm{C}_{18} \mathrm{H}_{34} \mathrm{ON} \\
\mathrm{C}_{16} \mathrm{H}_{32} \mathrm{~N}_{4}\end{array}$ & $\begin{array}{l}0.23 \\
1.58\end{array}$ & - & - & - \\
\hline 35.04 & 282.2791 & $\begin{array}{l}\mathrm{C}_{18} \mathrm{H}_{36} \mathrm{ON} \\
\mathrm{C}_{16} \mathrm{H}_{34} \mathrm{~N}_{4}\end{array}$ & $\begin{array}{l}0.18 \\
1.31\end{array}$ & - & - & - \\
\hline
\end{tabular}

\section{3: Conclusion}

Current industrial sample preparation methods for complex matrices such as wastewater products and biota are typically time-consuming and labour intensive, unsuitable for high throughput screening. The modified QuEChERS method offers an alternative sample preparation method which reduces the extraction time from hours to approximately 20 
minutes per sample. This method was successfully applied to a wastewater effluent, treated sludgecake and homogenised mussel tissue, with results showing the method's ability to extract five targeted pharmaceuticals and six biocides within the treated sludgecake samples, three biocides within effluent and four pharmaceuticals and four biocides within the mussel tissue samples using a dual ion trap and quadrupole platform. The calculated concentrations obtained significantly exceeded the suggested IDL values outlined in the CIP objectives, suggesting that significant bioaccumulation or replenishment due to insufficient removal during the wastewater treatment process may occur; a problem that will only continue to worsen without adequate remediation measures. Further implications of the presence of these compounds within the environment is the future risk to public health as a result of exposure of these compounds to the food chain through uptake from aquatic animals, either through contaminated wastewater effluent being released into watercourses or as a result of agricultural run-off from application of sludge to land.

The concentration of biocides reported within these fractions is also a concern; while alternative studies in Europe and China have shown the presence of QACs within sludge samples at equivalent concentration, biocides in effluent and biota have not been studied before. The detection of these QACs supports the need to identify these biocides as compounds of potential environmental concern and for monitoring through programmes such as CIP to inform UK Water Industry Research and EU policies, such as WFD.

A qualitative screen was successfully carried out on each of the environmental matrices of interest to this study, with results showing a selection of ions with similar predicted elemental formulas based upon their accurate mass. These predicted formulas suggest there could be a potential series of compounds, similar to the BACs, present within these sample types, indicating that they may be future compounds of interest for further work. 


\section{References}

[1] European Union. Directive 2013/39/EU of the European Parliament and of the council of 12 August 2013. Aberdeen: Official Journal of the European Union; 2013. Report No.: L226.

[2] Bhal SK. LogP-Making Sense of the Value. Advanced Chemistry Development, Inc. Available from: https://www.acdlabs.com/download/app/physchem/making_sense.pdf

[3] Berthod L, Roberts G, Whitley DC, Sharpe A, Mills GA.A solid-phase extraction method for rapidly determining the adsorption coefficient of pharmaceuticals in sewage sludge. Water Research. 2014; 67: 292-298.

[4] Boyd RK, Basic C, Bethem RA. Trace Quantitative Analysis by Mass Spectrometry. England: John Wiley \& Sons Ltd; 2008.

[5] Magnér J, Rosenqvist L, Magnus Rahmberg M, Graae L, Eliaeson K, Örtlund L, Fång J, Brorström-Lundén E. Fate of pharmaceutical residues - in sewage treatment and on farmland fertilized with sludge. IVL Swedish Environmental Research Institute Ltd. 2016: Report No.: B2264.

[6] Evans SE, Davies P, Lubben A, Kasprzyk-Hordern B. Determination of chiral pharmaceuticals and illicit drugs in wastewater and sludge using microwave assisted extraction, solid-phase extraction and chiral liquid chromatography coupled with tandem mass spectrometry. Analytica Chimica Acta. 2015; 882: 112-126.

[7] Miao X, Yang J, Metcalfe CD. Carbamazepine and Its Metabolites in Wastewater and in Biosolids in a Municipal Wastewater Treatment Plant. Environmental Science and Technology. 2005; 39 (19): 7469-7475.

[8] Gerba CP. Quaternary Ammonium Biocides: Efficacy in Application. Applied and Environmental Microbiology. 2015; 81(2): 464-469.

[9] Baracuda Pool Algaecide MSDS. Available from: http://www.baracuda.com.au/wpcontent/uploads/2015/09/X0629300_Baracuda-Pool-Algaecide.pdf

[10] European Union. Directive 2000/60/EC of the European Parliament and of the council of 23 October 2000. Aberdeen: Official Journal of the European Union; 2000. Report No.: L327.

[11] Silverman RB, Holladay MW. The Organic Chemistry of Drug Design and Drug Action. $3^{\text {rd }}$ Edition. USA: Academic Press; 2014: 19-122.

[12] Liu J, Lu GW, Sandoval M, Ciringh Y, Xue G, Jaeger D, Kompanik K, Jiao J, Gelotte KM. Determination of Benzalkonium Chloride Partition in Micelle Solutions Using Ultrafiltration Method. American Association of Pharmaceutical Scientists. 2009; 10 (4): 1216-1223.

[13] Ivanković T, Hrenović J. Surfactants in the Environment. Archives of Industrial Hygiene and Toxicology. 2010; 61: 95-110. 
[14] Zhu F, Wu X, Zhao L, Liu X, Qi J, Wang X, Wang J. Lipid profiling in sewage sludge. Water Research. 2017; 116: 149-158.

[15] Uno S, Yun JH, Kaneniwa M, Koyama J, Yamada H, Ikeda K. Lipid class and fatty acid composition of mussel, Mytilus trossulus, in Vancouver Harbor. PICES Scientific Report. 2001; 16: 43-46.

[16] Miller TH, McEneff GL, Brown RJ, Owen SF, Bury NR, Barron LP. Pharmaceuticals in the freshwater invertebrate, Gammarus pulex, determined using pulverised liquid extraction, solidphase extraction and liquid chromatography-tandem mass spectrometry. Science of the Total Environment. 2015; 511: 153-160.

[17] Alvarez-Muñoz D, Huerta B, Fernandez-Tejedor M, Rodríguez-Mozaz S, Barceló D. Multiresidue method for the analysis of pharmaceuticals and some of their metabolites in bivalves. Talanta. 2015; 136: 174-182.

[18] Maris P. Modes of action of disinfectants. Scientific and Technical Review of the Office International des Epizooties. 1995; 14(1): 47-55.

[19] Bhaskara VV, Middhab A, Tiwaria S, Shivakumara S. Liquid chromatography/tandem mass spectrometry method for quantitative estimation of polyethylene glycol 400 and its applications. Journal of Chromatography B. 2013; 926: 68- 76.

[20] Basit AW, Newton JM, Short MD, Waddington WA, Ell PJ, Lacey LF. The effect of polyethylene glycol 400 on gastrointestinal transit: implications for the formulation of poorlywater soluble drugs. Pharmaceutical Research. 2001; 18(8):1146-50.

[21] Bijlsma L, Sancho JV, Hernández F, Niessen WM. Fragmentation pathways of drugs of abuse and their metabolites based on QTOF MS/MS and MS(E) accurate-mass spectra. Journal of Mass Spectrometry. 2011; 46(9): 865-75. 


\section{Chapter 7: Conclusion and Further Work}

The Chemical Investigation Programme (CIP), established in 2009, is a UK based initiative aiming to identify and understand the prevalence of potential pollutants within wastewater samples, and to establish quality standards in wastewater similar to those outlined in the environmental quality standards directive (EQSD) (2008/105/EC). The initial CIP study was one of a number that directly fed into the amended environmental directive, 2013/39/EU, which also encompassed findings from the Water Framework Directive (WFD) and EQSD. This highlighted substances, including a selection of pharmaceuticals, of emerging concern to environmental contamination that are not yet subject to legislation but specified on a "watch list". Substances on the "watch list" (currently three pharmaceuticals) are considered to be hazardous and are therefore subject to a monitoring period, gathering data to determine the risk within the environment. In 2015, the second phase of the CIP study (CIP II) was launched to investigate these pharmaceuticals and other compounds identified as a potential environmental concern. This ongoing study focused on understanding their concentrations in environmental samples to inform policy on which technologies and processes provide the best contaminant removal at the most economical cost. There are a wide range of pollutants within the environment and pharmaceuticals are one such class of pollutant. Typically small molecules at relatively low abundance, the detection of these compounds within complex matrices, such as soils and wastewater, can be problematic. As $80 \%$ of treated sewage sludge is used as fertiliser on agricultural land, it is necessary to monitor whether this disposal route may have a negative impact on agricultural soil as a result of bioaccumulation of common pharmaceuticals. Previous research has also shown certain biocides are also prone to bioaccumulation within treated sewage sludge. Biocides and antibiotics share some similarities, whereby both classes of compounds control or destroy bacterial growth. The presence of these compounds within treated sewage sludge, and subsequently within agricultural soils could lead to an environment that is conducive to bacterial resistance.

Current recognised methods to analyse complex environmental matrices such as soil and wastewater effluent are generally multi-step analyses that are time consuming and laborious, with extractions taking up to 2 hours per sample, making them unsuitable for high-throughput analysis. These methods, when tested in-house (even on "simple" samples) have shown poor extraction of pharmaceuticals with poor repeatability. Given the disadvantages of these approaches, an alternative method capable of preparing these complex samples with minimal 
matrix effects that may adversely influence the measured signal, repeatable recovery and performance involving less time and resources is required.

The main aim of this project was to develop and evaluate a single modified QuEChERS sample preparation method suitable for detection and quantitation of compounds of interest to CIP within a selection of environmental matrices of interest to WFD. Sample types investigated were soil, treated effluent, treated sludgecake and homogenised biota (mussel tissue). All extracted samples were analysed using LC-MS with an internal standard approach to quantitation.

\section{1: Quantitative LC-MS Method Fitness for Purpose}

A quantitative method for detecting and measuring a suite of 10 pharmaceuticals and 4 internal standards was successfully developed using a ZQ4000 single quadrupole mass spectrometer. This method was adapted to include 6 quaternary ammonium compounds with 1 internal standard (QACs) using an LCQ ion trap mass spectrometer. Both methods were successfully evaluated using recognised performance criteria for analytical testing. The evaluation confirmed the while the pharmaceutical method was suitable for accurate and precise quantitation for concentrations between $15-400 \mathrm{ng} / \mathrm{mL}$, the performance of the biocide method was limited, and would benefit from additional development to ensure that the best possible data for reliable quantitation could be achieved. The method was evaluated for QAC concentrations between 8-80 $\mathrm{ng} / \mathrm{mL}$, and deemed fit for purpose for this initial "proof-of-concept" study, with IDL values determined empirically to be less than $1 \mathrm{ng} / \mathrm{mL}$ and $2 \mathrm{ng} / \mathrm{mL}$ for pharmaceuticals and QACs, respectively.

\section{2: Modified QuEChERS Extraction Fitness for Purpose}

A modified QuEChERS sample preparation method was developed in collaboration with Biotage GB. The method uses a custom extraction mixture of $4 \mathrm{~g}$ magnesium sulphate $\left(\mathrm{MgSO}_{4}\right)$ and $1.5 \mathrm{~g}$ sodium acetate and an EN dSPE kit comprised of magnesium sulphate and primary secondary amine (PSA). This method was successfully used to extract both suites of pharmaceuticals and biocides from water and soil samples. The matrix effects and extraction recovery of each compound were characterised within water and soil samples. The matrix effects observed with the modified QuEChERS method were greatly improved versus the standardised methods, with recorded percentages for each compound around 100\%, showing only slight matrix enhancement for some compounds. Although recovery was less 
than the traditional methods reported in the literature, the QuEChERS extraction and preparation method significantly reduced the time and reagents required by traditional sample preparation methods such as liquid-liquid, and solid-phase extractions.

\section{3: Qualitative and Quantitative Analysis within Environmental Matrices}

The modified QuEChERS extraction method was tested on more complex environmental samples; treated effluent, treated sludgecake and homogenised biota (mussel tissue). The compounds present within each sample were confirmed using their chromatographic retention time, precursor $m / z$ and any fragment $m / z$, with the latter being used to confirm identity with accurate mass data. Each of the QAC biocides were successfully detected and quantified within the summer and winter sludgecake samples, with $50 \%$ and $80 \%$ of the pharmaceutical suite detected respectively. Two pharmaceuticals were detected at a quantifiable concentration within the winter sludgecake sample, citalopram and fluoxetine, the most abundant of which, citalopram, was measured at $995.31 \mu \mathrm{g} / \mathrm{kg}$, which is slightly higher than previous studies. The observed concentration of biocide were much higher, with concentrations measured in the $\mathrm{mg} / \mathrm{kg}$ range, the highest being BAC-C12 at $70.37 \mathrm{mg} / \mathrm{kg}$ (within the summer sample), which is much higher than previous studies, and the concentration of DDMA and HDTMA (26.81 and $41.39 \mathrm{mg} / \mathrm{kg}$, respectively) have not been seen at this level. For both preparations of homogenised biota (wet and lyophilised) only 2 pharmaceuticals and 4 QACs were detected, with measured concentrations of biocides much lower than within the sludgecake samples. Although some work has been carried out to detect biocides in wastewater sludge, their presence and concentration has not been extensively studied within biota previously. The quantitation of compounds of interest from the treated effluent was more challenging; the sample was more dilute, therefore the pharmaceuticals detected were below the IDL, and the BAC compounds detected were below $5 \mathrm{ng} / \mathrm{mL}$.

A qualitative screen was successfully performed with each of the sample matrices and in addition to confirming the identity of the target compounds, a selection of ions detected $(\mathrm{m} / \mathrm{z}$ $150.0911,228.2320$ and 430.2435) were common to all three matrices. Identity of these compounds could be useful to further understand the extent of bioaccumulation/replenishment of compounds other than pharmaceuticals, within multiple wastewater fractions. 


\section{4: Impact of the Findings}

The developed QuEChERS method has shown that it is capable of extracting both pharmaceuticals and biocides from multiple environmental matrices. This is the first study to demonstrate the ability of a QuEChERS sample preparation method in co-extracting pharmaceuticals and QACs from a single sample preparation method, in these environmental matrices. The WFD and the CIP programme have specified low limits of detection for potential pollutants of interest with suggested IDLs at $0.01 \mu \mathrm{g} / \mathrm{L}$ ( or $10 \mathrm{pg} / \mathrm{mL}$ ), challenging the quantitative ability for most analytical instrumentation. However, from our initial screen it was apparent that perceived concentrations were considerably higher. Both the ZQ4000 and the LCQ ion trap are low-resolution mass spectrometers that have shown sufficient sensitivity for the selected pharmaceuticals and QAC biocides respectively, with good levels of quantitative accuracy and precision. It has detected and quantified 7 pharmaceuticals and 6 biocides and confirmed the higher than expected concentrations in samples during different seasons (suggesting all-year round release), their presence in effluent as well as the solid fraction of wastewater, and a transfer to biota, which along with the detection of QACs in these sample, could have a potentially greater impact on public health and ecological risk if released into the environment. This project has also created a new product line for Biotage $\mathrm{GB}$, in the modified QuEChERS extraction mixture, which is not only suitable for treated sludgecake, but has also been successfully applied to soil, treated effluent and homogenised biota samples. The loss in recovery from the extraction, although significant for some compounds, does not outweigh the benefit of reducing preparation time from hours to 20 minutes per sample, reducing solvent usage from approximately $200 \mathrm{~mL}$ to $15 \mathrm{~mL}$, and eliminating the need for a complex vacuum or distillation apparatus as seen in current regulatory methods (NRW and EPA). The concentrations at which both the pharmaceuticals and the biocides were detected in the sludgecake at high $\mu \mathrm{g} / \mathrm{kg}$ and $\mathrm{mg} / \mathrm{kg}$ contributes to the CIP II investigation and also the investigation by which biocides are contributing to antibiotic resistance. This, with the quantities at which these QACs were present within effluent and biota samples, supports the need for QACs to be recognised within CIP and the WFD as a compound of emerging concern.

\section{5: Further Work}

Although the LC-MS method and QuEChERS extraction were fit for purpose, both can be improved upon to detect and quantify the suite of pharmaceuticals and biocides at even lower 
concentrations. While the analytical method for the pharmaceutical suite was deemed suitable for quantitation, the use of isotopically labelled internal standards and performing a complete method validation would further improve the validity of this method for future research and publication opportunities. Amendments to the quantitative method for the suite of biocides would also be beneficial in improving the reliability of the data; firstly, additional segmenting of the method could be investigated to improve the number of data points across the chromatographic peak for quantitation and secondly, the calibration range could be considered to help improve the linearity by reducing the concentration of the top standard to prevent any potential signal saturation. The use of alternative instrumentation could increase the ability to quantify at the lower limits of detection with improved precision and accuracy, which subsequently could lead to further improvement of the modified QuEChERS method. For example, by using more sensitive instrument, such as a triple quadrupole or quadrupole time-of-flight mass spectrometer, a dilution factor could be introduced into the QuEChERS protocol, further reducing matrix effects and providing a cleaner extract for analysis. Alternative instrumentation could also aid in the identification of target compounds, either using a high resolution instrument to acquire accurate mass information, or by using a triple quadrupole mass spectrometer, which is a more sensitive instrument than the ZQ4000 or LCQ used in this study, and can also provide MS/MS data, which would be particularly useful for the identification of the suite of pharmaceuticals. The modified QuEChERS method, while successfully applied to the environmental matrices investigated, could be further developed. Smaller sample volumes, or a miniaturisation of the protocol as a whole could be investigated to further reduce the operational costs further and reduce sample handling risks associated with more complex samples. The presence of both pharmaceuticals and biocides within the local sludgecake is the first step in understanding the effect of these compounds in the wider environment. While the method was only used to characterise the extraction procedure in soil, a quantitative analysis of agricultural soils could be useful to determine whether these compounds of interest are leaching into the soils from the sludgecake used a fertiliser. This information may also be used to underpin studies in mapping genetic change in soil bacteria and the potential development in antimicrobial resistance. The presence of both pharmaceuticals and QACs within the biota samples tested also highlights an important aspect in determining the main route of exposure to these animals. Initially it was hypothesised that uptake of contaminant may be occurring via the filtering of contaminated waters, however, the concentrations of pharmaceutical and QAC 
within wastewater effluent were found to be low, therefore suggesting an alternative route of exposure, such as absorption via the sediment within the biota beds could be the cause, due to the lipophilic nature of these compounds. An investigation into the presence of QACs in the environment could be useful to determine how they relate to antibiotic resistant bacteria, which could be used to inform policy makers about the public and industrial use of quaternary ammonium biocides, and how wastewater and sludge are treated for these emerging pollutants. 


\section{Appendix 1.1}

A preliminary chromatogram of treated sludgecake obtained in-house and a summary of potential compounds detected. Data was analysed using a combination of Mass Frontier (to provide small-molecule structural elucidation) and Xcalibur 2.0.7 software.

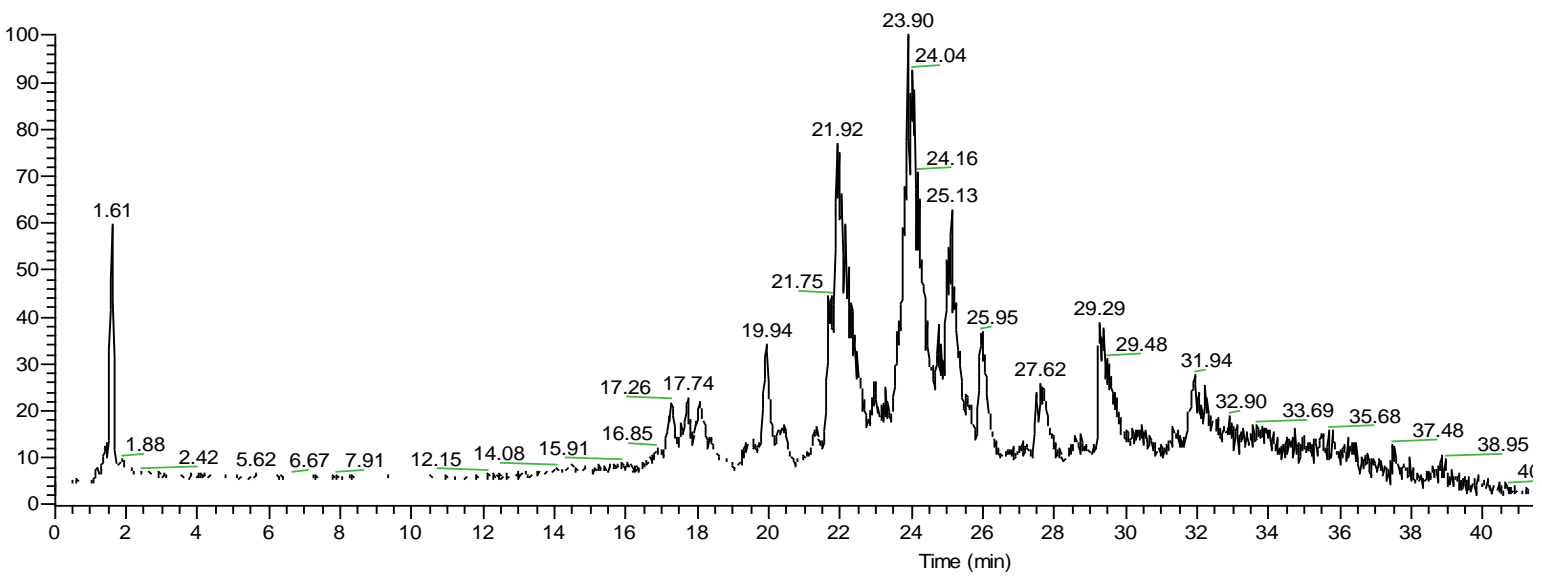

\begin{tabular}{|c|c|c|c|c|}
\hline $\begin{array}{c}\text { Retention } \\
\text { Time (min) }\end{array}$ & $\begin{array}{c}\text { Ion of Interest } \\
(\mathbf{m} / \mathbf{z})\end{array}$ & Possible ID & Formula & $\begin{array}{c}\text { Error } \\
\text { (ppm) }\end{array}$ \\
\hline 1.71 & 152.07 & Acetaminophen & $\mathrm{C}_{8} \mathrm{H}_{9} \mathrm{NO}_{2}$ & -3.65 \\
\hline 14.67 & 260.164 & Propranolol & $\mathrm{C}_{16} \mathrm{H}_{21} \mathrm{NO}_{2}$ & -2.865 \\
\hline 15.24 & 505.324 & Dipyridamole & $\mathrm{C}_{24} \mathrm{H}_{40} \mathrm{~N}_{8} \mathrm{O}_{4}$ & -0.907 \\
\hline 15.57 & 325.171 & Citalopram & $\mathrm{C}_{20} \mathrm{H}_{21} \mathrm{FN}_{2} \mathrm{O}$ & -0.794 \\
\hline 15.58 & 267.185 & Cyclizine & $\mathrm{C}_{18} \mathrm{H}_{22} \mathrm{~N}_{2}$ & -1.479 \\
\hline 16.57 & 407.196 & Carvedilol & $\mathrm{C}_{24} \mathrm{H}_{26} \mathrm{~N}_{2} \mathrm{O}_{4}$ & -1.114 \\
\hline 17.00 & 455.291 & Verapamil & $\mathrm{C}_{27} \mathrm{H}_{38} \mathrm{~N}_{2} \mathrm{O}_{4}$ & 0.804 \\
\hline 17.24 & 515.2431 & Telmisartan & $\mathrm{C}_{33} \mathrm{H}_{30} \mathrm{~N}_{4} \mathrm{O}_{2}$ & -0.683 \\
\hline 17.32 & 278.1897 & Amitriptyline & $\mathrm{C}_{20} \mathrm{H}_{23} \mathrm{~N}$ & -0.166 \\
\hline 18.22 & 383.152 & Loratadine & $\mathrm{C}_{22} \mathrm{H}_{23} \mathrm{ClN}_{2} \mathrm{O}_{2}$ & -1.232 \\
\hline 21.92 & 304.2993 & BAC-C12 & $\mathrm{C}_{21} \mathrm{H}_{38} \mathrm{~N}$ & -0.777 \\
\hline 23.90 & 332.3303 & BAC-C14 & $\mathrm{C}_{23} \mathrm{H}_{42} \mathrm{~N}$ & -0.877 \\
\hline 23.90 & 284.3305 & HDTMA & $\mathrm{C}_{19} \mathrm{H}_{42} \mathrm{~N}$ & -0.737 \\
\hline 25.13 & 326.377 & DDMA & $\mathrm{C}_{22} \mathrm{H}_{48} \mathrm{~N}$ & -0.627 \\
\hline 25.95 & 360.3617 & BAC-C16 & $\mathrm{C}_{25} \mathrm{H}_{46} \mathrm{~N}$ & -0.537 \\
\hline 25.95 & 312.3621 & Octadecyltrimethylammonium & $\mathrm{C}_{21} \mathrm{H}_{46} \mathrm{~N}$ & -0.177 \\
\hline 27.60 & 388.3932 & BAC-C18 & $\mathrm{C}_{27} \mathrm{H}_{50} \mathrm{~N}$ & -0.761 \\
\hline 29.29 & 368.425 & Trioctylmethylammonium cation & $\mathrm{C}_{25} \mathrm{H}_{54} \mathrm{~N}$ & -0.487 \\
\hline 29.69 & 368.425 & Trioctylmethylammonium cation & $\mathrm{C}_{25} \mathrm{H}_{54} \mathrm{~N}$ & -0.487 \\
\hline 30.89 & 315.231 & Delta-1-THC - also Delta-9-THC & $\mathrm{C}_{21} \mathrm{H}_{30} \mathrm{O}_{2}$ & -3.25 \\
\hline
\end{tabular}




\section{Appendix 2.1}

A summary of pharmaceutical data used in this study organised in order of $\log \mathrm{P}$. All pKa and $\log \mathrm{P}$ da

\begin{tabular}{|c|c|c|c|}
\hline Pharmaceuticals & Ion & Formula & Structure \\
\hline Acetaminophen & {$[\mathrm{M}+\mathrm{H}]^{+}=152$} & $\mathbf{C}_{8} \mathbf{H}_{9} \mathbf{N O}_{2}$ & \\
\hline Acetaminophen-(methyl)- $\mathrm{d}_{3}$ & {$[\mathrm{M}+\mathrm{H}]^{+}=155$} & $\mathrm{C}_{8} \mathrm{H}_{6} \mathbf{D}_{3} \mathrm{NO}_{2}$ & \\
\hline $\begin{array}{l}\text { Benzyldimethyldodecylammonium } \\
\text { Chloride (BAC-C12) }\end{array}$ & {$[\mathrm{M}-\mathrm{Cl}]^{+}=304$} & $\mathbf{C}_{21} \mathbf{H}_{38} \mathbf{N}$ & \\
\hline $\begin{array}{l}\text { Hexadecyltrimethylammonium } \\
\text { Chloride (HDTMA) }\end{array}$ & {$[\mathrm{M}-\mathrm{Cl}]^{+}=284$} & $\mathbf{C}_{19} \mathbf{H}_{42} \mathbf{N}$ & \\
\hline Citalopram Hydrobromide & {$[\mathrm{M}-\mathrm{HBr}+\mathrm{H}]^{+}=325$} & $\mathbf{C}_{20} \mathbf{H}_{21} \mathrm{FN}_{2} \mathbf{O}$ & \\
\hline $\begin{array}{l}\text { Didecyldimethylammonium Bromide } \\
\text { (DDMA) }\end{array}$ & {$[\mathrm{M}-\mathrm{Br}]^{+}=326$} & $\mathbf{C}_{22} \mathbf{H}_{48} \mathbf{N}$ & \\
\hline $\begin{array}{l}\text { Benzyldimethyltetradecylammonium } \\
\text { Chloride (BAC-C14) }\end{array}$ & {$[\mathrm{M}-\mathrm{Cl}]^{+}=332$} & $\mathbf{C}_{23} \mathbf{H}_{42} \mathbf{N}$ & \\
\hline $\begin{array}{l}\mathrm{d}_{7} \text {-Benzyldimethyltetradecylammonium } \\
\text { Chloride }\left(\mathrm{BAC}-\mathrm{C} 14-\mathrm{d}_{7}\right)\end{array}$ & {$[\mathrm{M}-\mathrm{Cl}]^{+}=339$} & $\mathbf{C}_{23} \mathbf{H}_{35} \mathbf{D}_{\underline{7}} \mathbf{N}$ & \\
\hline
\end{tabular}


Rachel Townsend

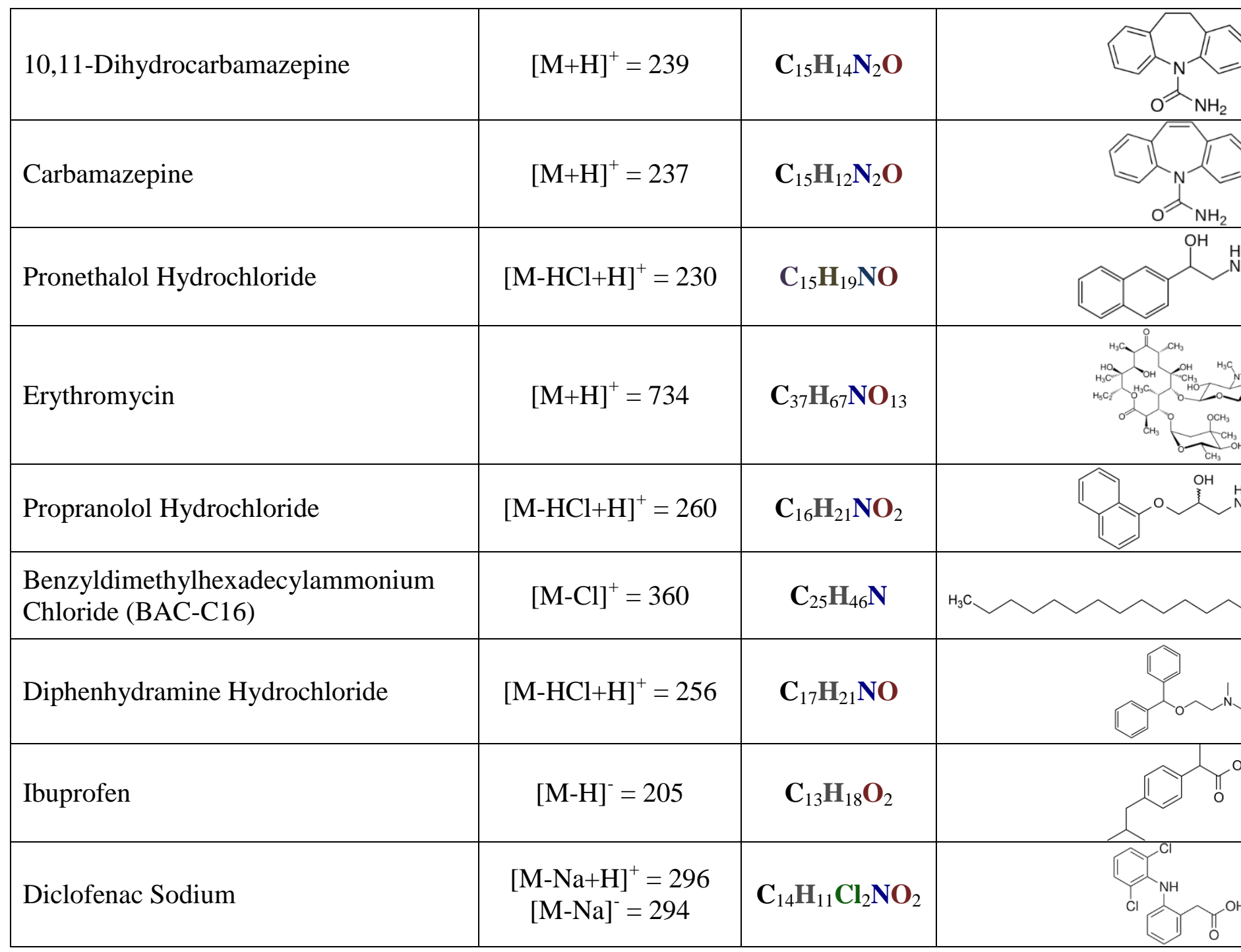


Rachel Townsend

\begin{tabular}{|c|c|c|}
\hline Fluoxetine Hydrochloride & {$[\mathrm{M}-\mathrm{HCl}+\mathrm{H}]^{+}=310$} & $\mathbf{C}_{17} \mathbf{H}_{18} \mathbf{F}_{3} \mathbf{N O}$ \\
\hline Stearalkonium Chloride (BAC-C18) & {$[\mathrm{M}-\mathrm{Cl}]^{+}=388$} & $\mathbf{C}_{27} \mathbf{H}_{50} \mathbf{N}$ \\
\hline Talopram Hydrochloride & {$[\mathrm{M}-\mathrm{HCl}+\mathrm{H}]^{+}=296$} & $\mathbf{C}_{20} \mathbf{H}_{25} \mathbf{N O}$ \\
\hline Loratadine & {$[\mathrm{M}+\mathrm{H}]^{+}=383$} & $\mathbf{C}_{22} \mathrm{H}_{23} \mathrm{CIN}_{2} \mathbf{O}_{2}$ \\
\hline
\end{tabular}




\section{Appendix 3.1}

Mass spectra obtained during direct infusion for each pharmaceutical. Inset: a structure and a data table detailing the molecular formula, monoisotopic mass, precursor ion observed and the optimum cone voltage $(\mathrm{CV})$ for each compound. All structures were drawn using ChemDraw ${ }^{\circledR}$ software, and the data was taken from the ChemSpider database.

\section{Acetaminophen-(methyl)-d 3}

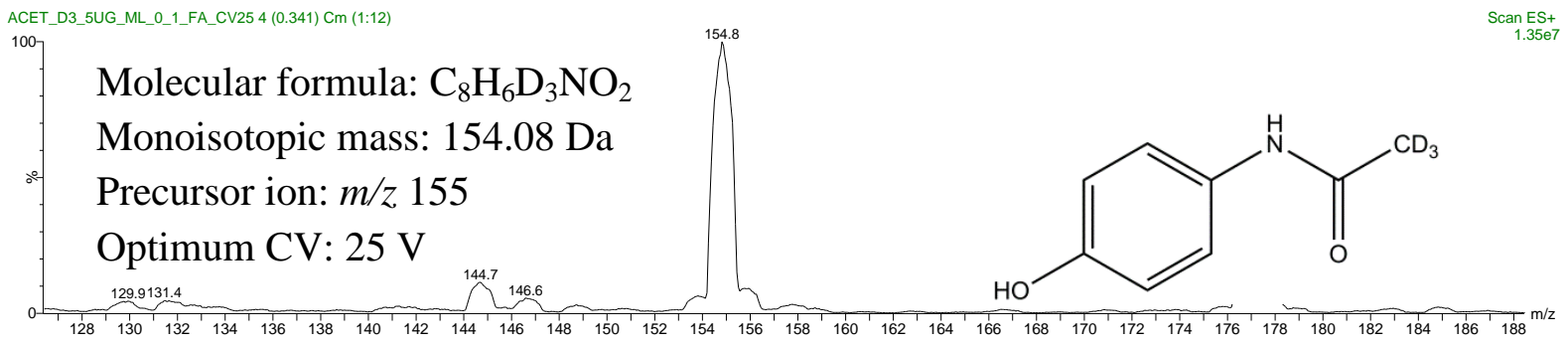

\section{Atenolol}

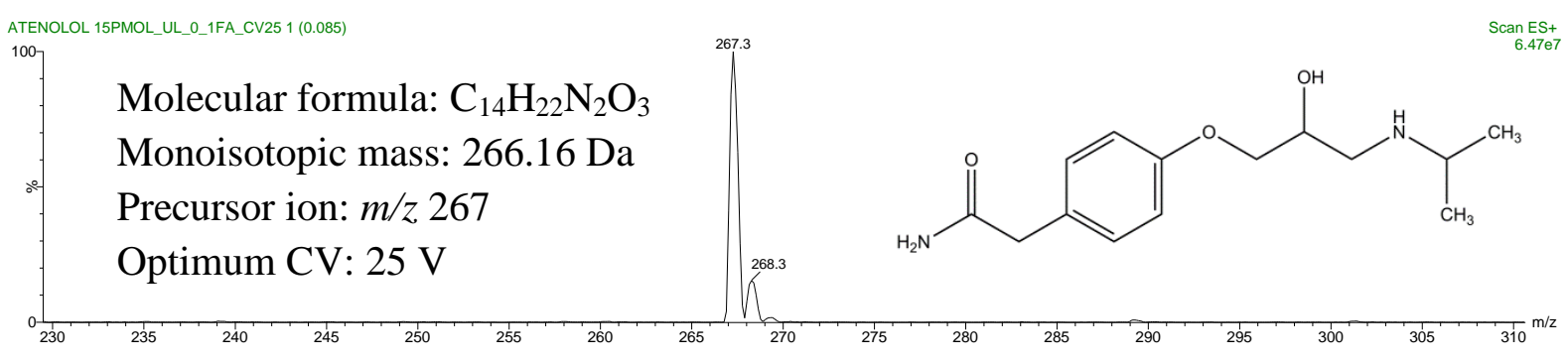

\section{Carbamazepine}

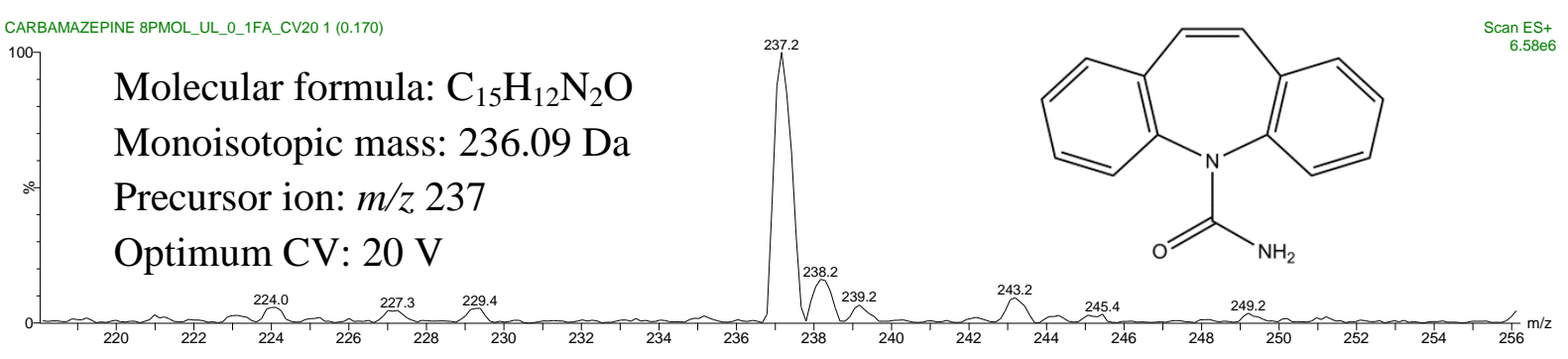




\section{Diclofenac}

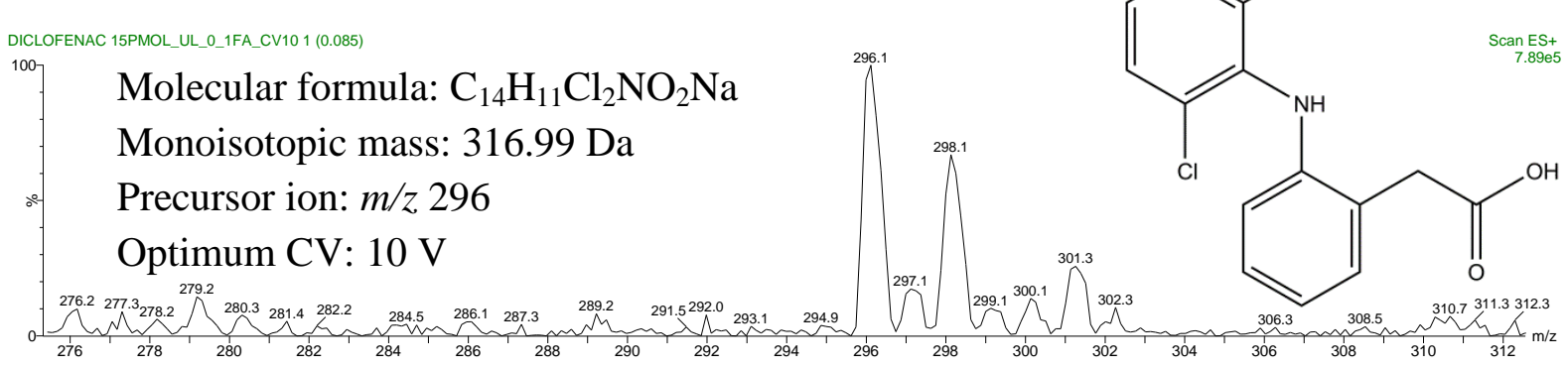

\section{Erythromycin}

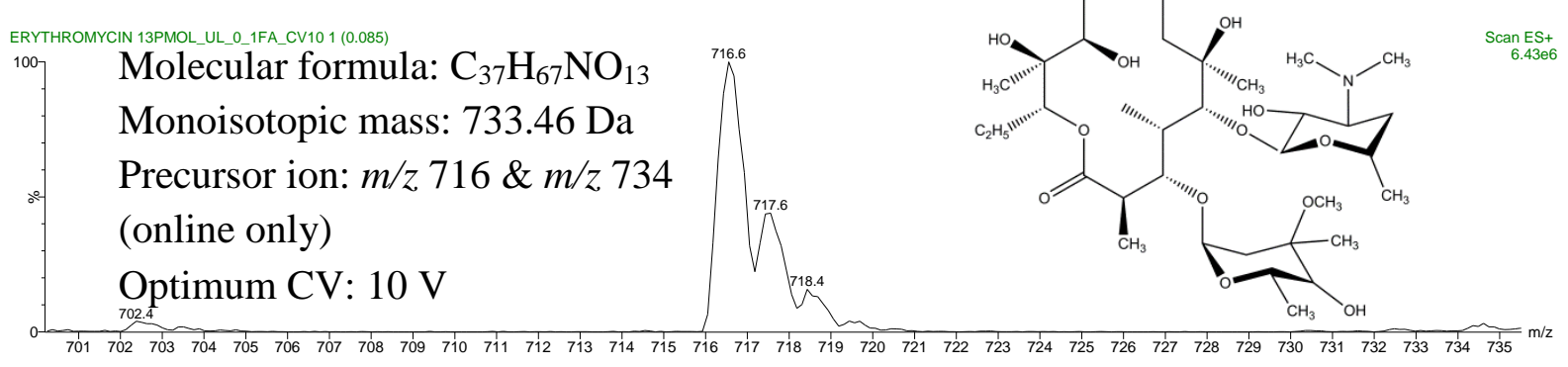

\section{Fluoxetine}

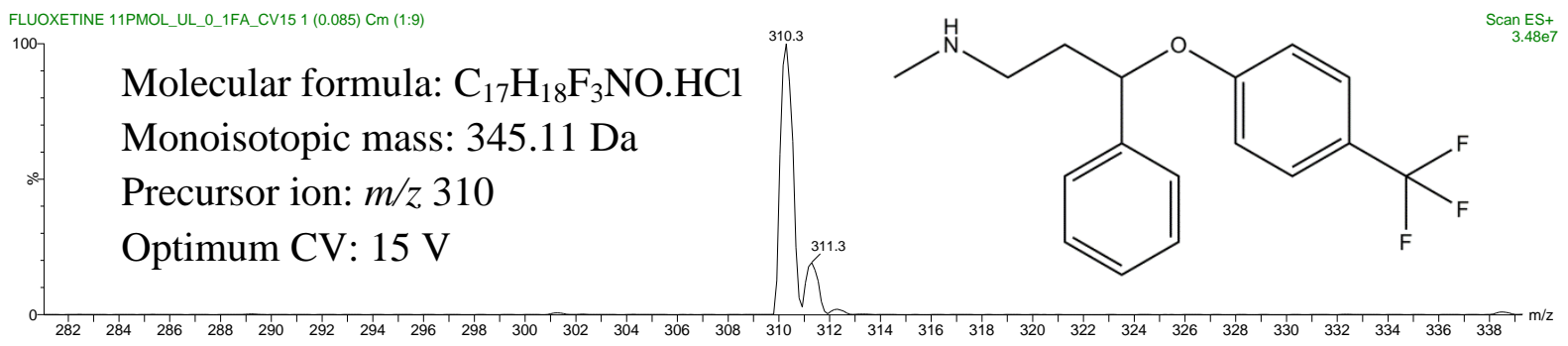

\section{Loratadine}

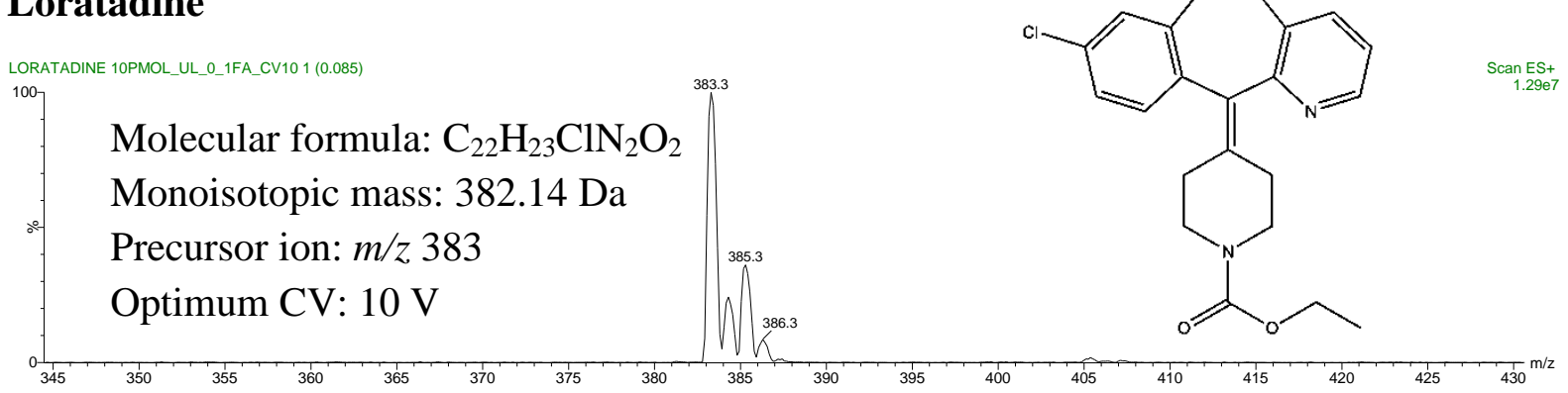




\section{Pronethalol}

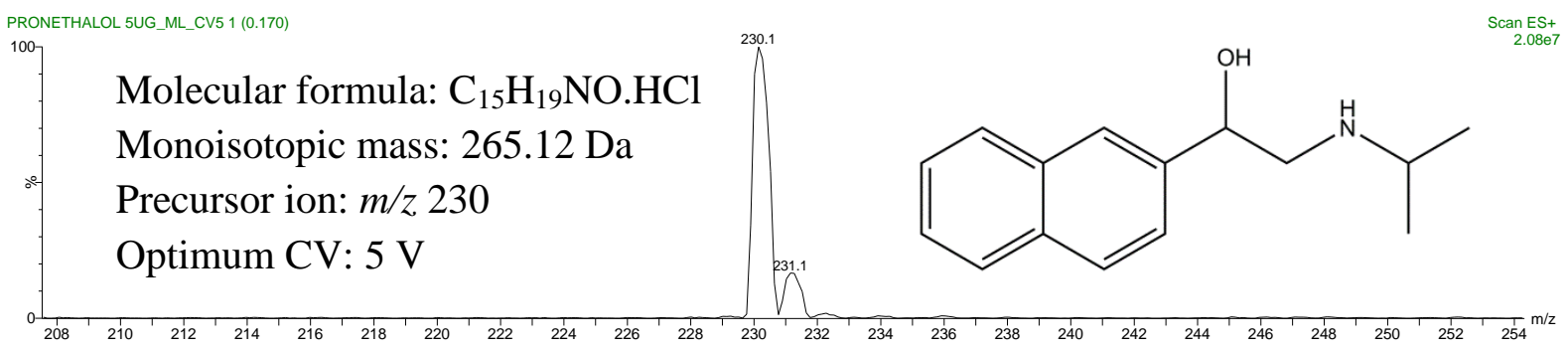

\section{Propranolol}

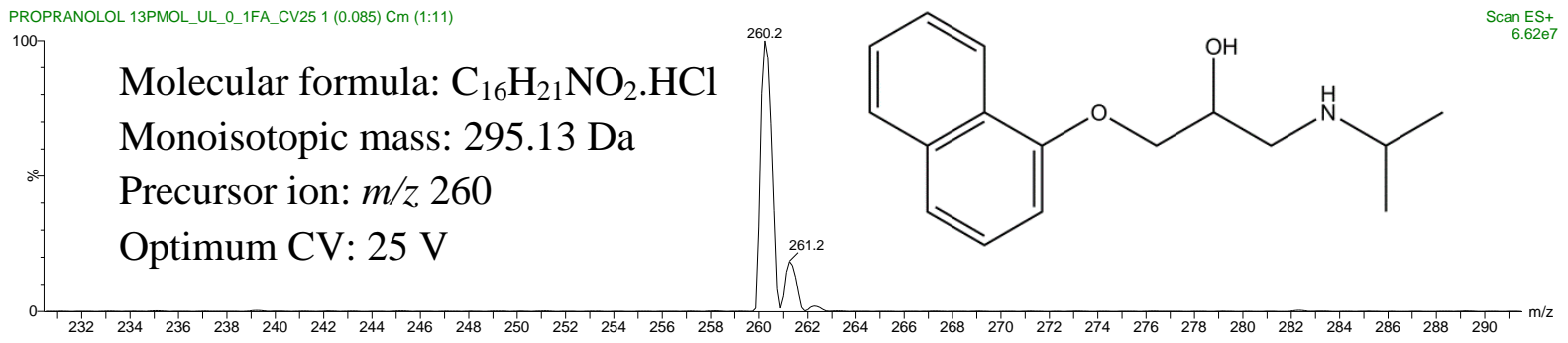

\section{0,11-Dihydrocarbamazepine}

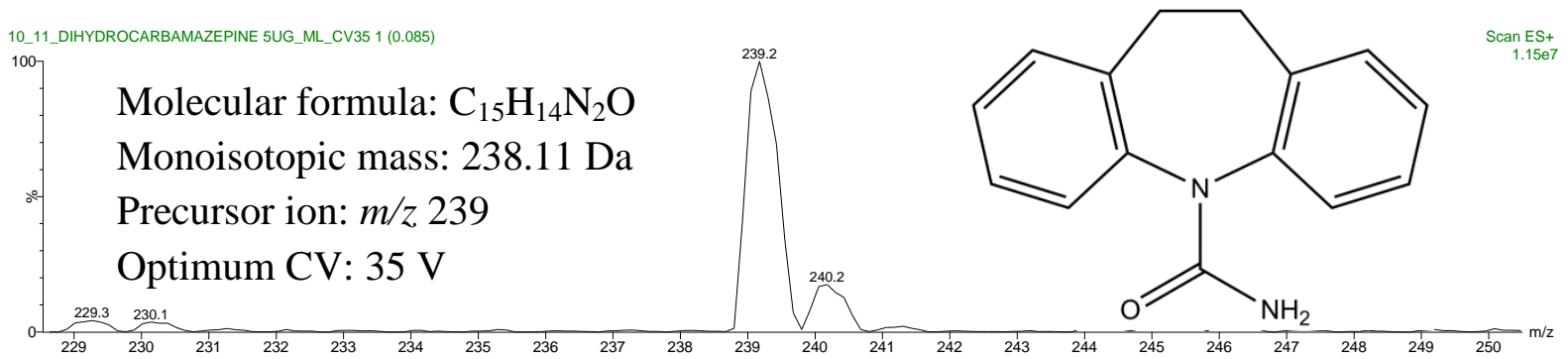




\section{Appendix 3.2}

A table summarising the injection repeatability data obtained using the Xbridge C18 column.

\begin{tabular}{|c|c|c|c|}
\hline \multirow[b]{2}{*}{$m / z$} & \multicolumn{2}{|c|}{ Peak Area } & \multirow{2}{*}{$\begin{array}{c}\begin{array}{c}\text { Critical } \\
\text { Value } 4.484\end{array} \\
\text { F-Test }\end{array}$} \\
\hline & $\begin{array}{c}\text { \% CV Day } \\
1\end{array}$ & $\begin{array}{c}\text { \% CV Day } \\
2\end{array}$ & \\
\hline $\begin{array}{c}230 \\
\text { Pronethalol }\end{array}$ & 12.23 & 15.20 & 1.45 \\
\hline $\begin{array}{c}260 \\
\text { Propranolol }\end{array}$ & 6.69 & 11.73 & 4.91 \\
\hline $\begin{array}{c}256 \\
\text { Diphenhydramine }\end{array}$ & 6.12 & 13.33 & 4.80 \\
\hline $\begin{array}{c}325 \\
\text { Citalopram }\end{array}$ & 7.87 & 22.51 & 5.64 \\
\hline $\begin{array}{c}734 \\
\text { Erythromycin }\end{array}$ & 15.70 & 8.59 & 4.98 \\
\hline $\begin{array}{c}237 \\
\text { Carbamazepine } \\
\end{array}$ & 7.15 & 8.01 & 2.51 \\
\hline $\begin{array}{c}239 \\
10,11-\mathrm{DHC}\end{array}$ & 7.16 & 11.68 & 2.31 \\
\hline $\begin{array}{c}310 \\
\text { Fluoxetine }\end{array}$ & 6.01 & 15.36 & 5.68 \\
\hline $\begin{array}{c}296 \\
\text { Talopram }\end{array}$ & 10.81 & 11.87 & 4.39 \\
\hline $\begin{array}{c}383 \\
\text { Loratadine }\end{array}$ & 6.84 & 8.37 & 4.27 \\
\hline $\begin{array}{c}296 \\
\text { Diclofenac }\end{array}$ & 5.34 & 9.98 & 3.31 \\
\hline
\end{tabular}




\section{Appendix 3.3}

A table summarising the reproducibility data obtained using the Xselect HSS T3 column.

\begin{tabular}{|c|c|c|c|c|}
\hline \multirow[b]{2}{*}{$m / z$} & \multicolumn{2}{|c|}{$\begin{array}{c}\text { Adjusted Retention } \\
\text { Time }\end{array}$} & \multirow{2}{*}{$\begin{array}{c}F_{(9,5)} \\
6.681 \\
\text { F-Test }\end{array}$} & \multirow{2}{*}{$\begin{array}{c}F_{(5,9)} \\
4.484 \\
\text { F-Test }\end{array}$} \\
\hline & $\begin{array}{c}\text { \% CV Day } \\
1\end{array}$ & $\begin{array}{c}\text { \% CV Day } \\
2\end{array}$ & & \\
\hline $\begin{array}{c}155 \\
\text { Acet-d }\end{array}$ & 2.84 & 4.24 & & 2.42 \\
\hline $\begin{array}{c}152 \\
\text { Acetaminophen }\end{array}$ & 2.84 & 5.50 & & 4.00 \\
\hline $\begin{array}{c}230 \\
\text { Pronethalol }\end{array}$ & 1.59 & 0.40 & 16.66 & \\
\hline $\begin{array}{c}260 \\
\text { Propranolol }\end{array}$ & 1.00 & 0.28 & 13.26 & \\
\hline $\begin{array}{c}256 \\
\text { Diphenhydramine }\end{array}$ & 0.86 & 0.29 & 9.02 & \\
\hline $\begin{array}{c}325 \\
\text { Citalopram }\end{array}$ & 0.78 & 0.36 & 4.87 & \\
\hline $\begin{array}{c}734 \\
\text { Erythromycin }\end{array}$ & 0.75 & 0.23 & 11.18 & \\
\hline $\begin{array}{c}237 \\
\text { Carbamazepine }\end{array}$ & 0.74 & 0.31 & 5.85 & \\
\hline $\begin{array}{c}239 \\
10,11-\mathrm{DHC} \\
\end{array}$ & 0.73 & 0.23 & 10.60 & \\
\hline $\begin{array}{c}310 \\
\text { Fluoxetine }\end{array}$ & 0.56 & 0.24 & 5.41 & \\
\hline $\begin{array}{c}296 \\
\text { Talopram }\end{array}$ & 0.68 & 0.26 & 7.10 & \\
\hline $\begin{array}{c}383 \\
\text { Loratadine }\end{array}$ & 0.55 & 0.18 & 9.16 & \\
\hline $\begin{array}{c}296 \\
\text { Diclofenac } \\
\end{array}$ & 0.38 & 0.23 & 2.86 & \\
\hline $\begin{array}{c}205 \\
\text { Ibuprofen }\end{array}$ & 0.51 & 0.19 & 7.60 & \\
\hline
\end{tabular}




\section{Appendix 3.4}

Summary of data obtained to evaluate the heteroscedasticity of the calibration standards using the Xselect HSS T3 column.

\begin{tabular}{|l|c|c|c|c|c|}
\hline \multirow{2}{*}{ Compound } & \multicolumn{2}{|c|}{$\begin{array}{c}\text { Variance of Relative } \\
\text { Response Factor }\end{array}$} & \multirow{2}{*}{ F-Calc } & $\begin{array}{c}\text { F-Stat (2,2, } \\
\mathbf{0 . 9 5} \text { one } \\
\text { tailed }\end{array}$ & Homoscedastic? \\
\cline { 2 - 3 } & $\mathbf{S 1}$ & $\mathbf{S 9}$ & & 19 & No \\
\hline Acetaminophen & 0.000019 & 0.009825 & 517.1228 & 19 & No \\
\hline Carbamazepine & $3.33 \mathrm{E}-07$ & 0.004446 & 13339 & 19 & No \\
\hline Citalopram & $1.33 \mathrm{E}-06$ & 0.000224 & 168.25 & 19 & No \\
\hline Diclofenac & $4.33 \mathrm{E}-06$ & $5.033 \mathrm{E}-05$ & 11.6154 & 19 & No \\
\hline Diphenhydramine & $2.33 \mathrm{E}-06$ & 0.003046 & 1305.571 & 19 & No \\
\hline Erythromycin & $1.82 \mathrm{E}-09$ & $2.03 \mathrm{E}-05$ & 11202.9 & 19 & No \\
\hline Fluoxetine & $2.33 \mathrm{E}-06$ & 0.000625 & 268 & 19 & No \\
\hline Ibuprofen & $3.33 \mathrm{E}-07$ & $3.1 \mathrm{E}-05$ & 93 & 19 & No \\
\hline Loratadine & $8.24 \mathrm{E}-08$ & 0.006922 & 84008.55 & 19 & No \\
\hline Propranolol & 0.00014 & 0.14724 & 1049.219 & & \\
\hline
\end{tabular}




\section{Appendix 3.5}

Calibration graphs for each compound in the suite, generated using MassLynx 4.1 software. These graph statistics for the Xselect HSS T3 column.
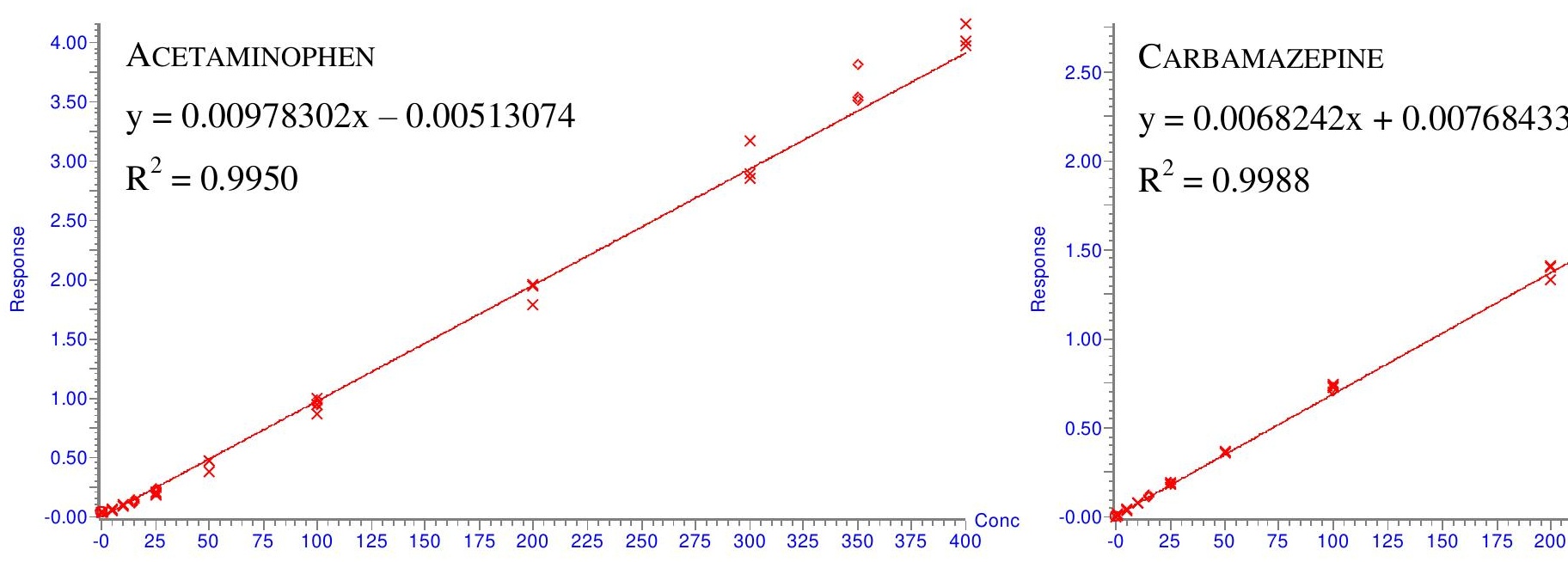

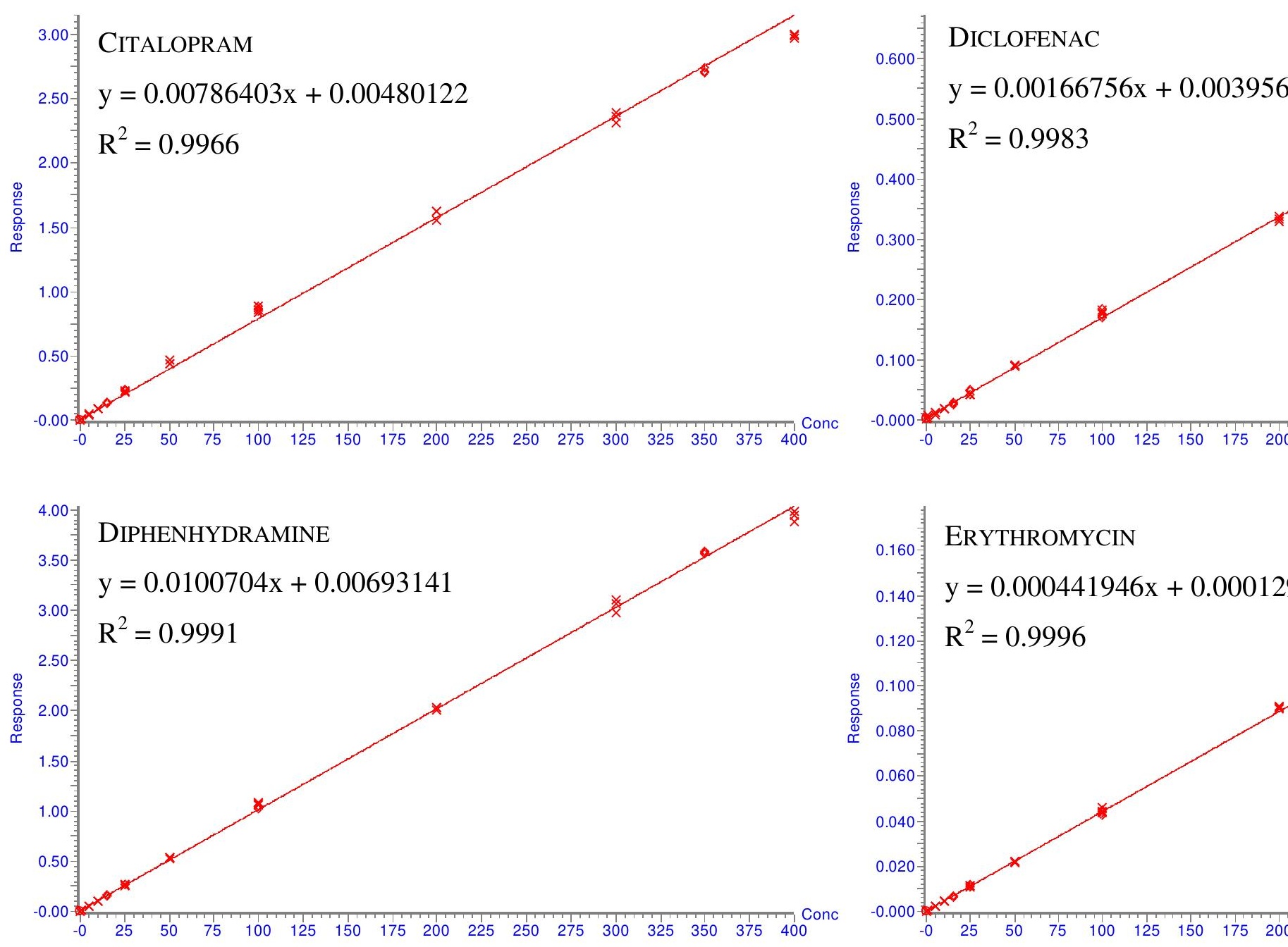

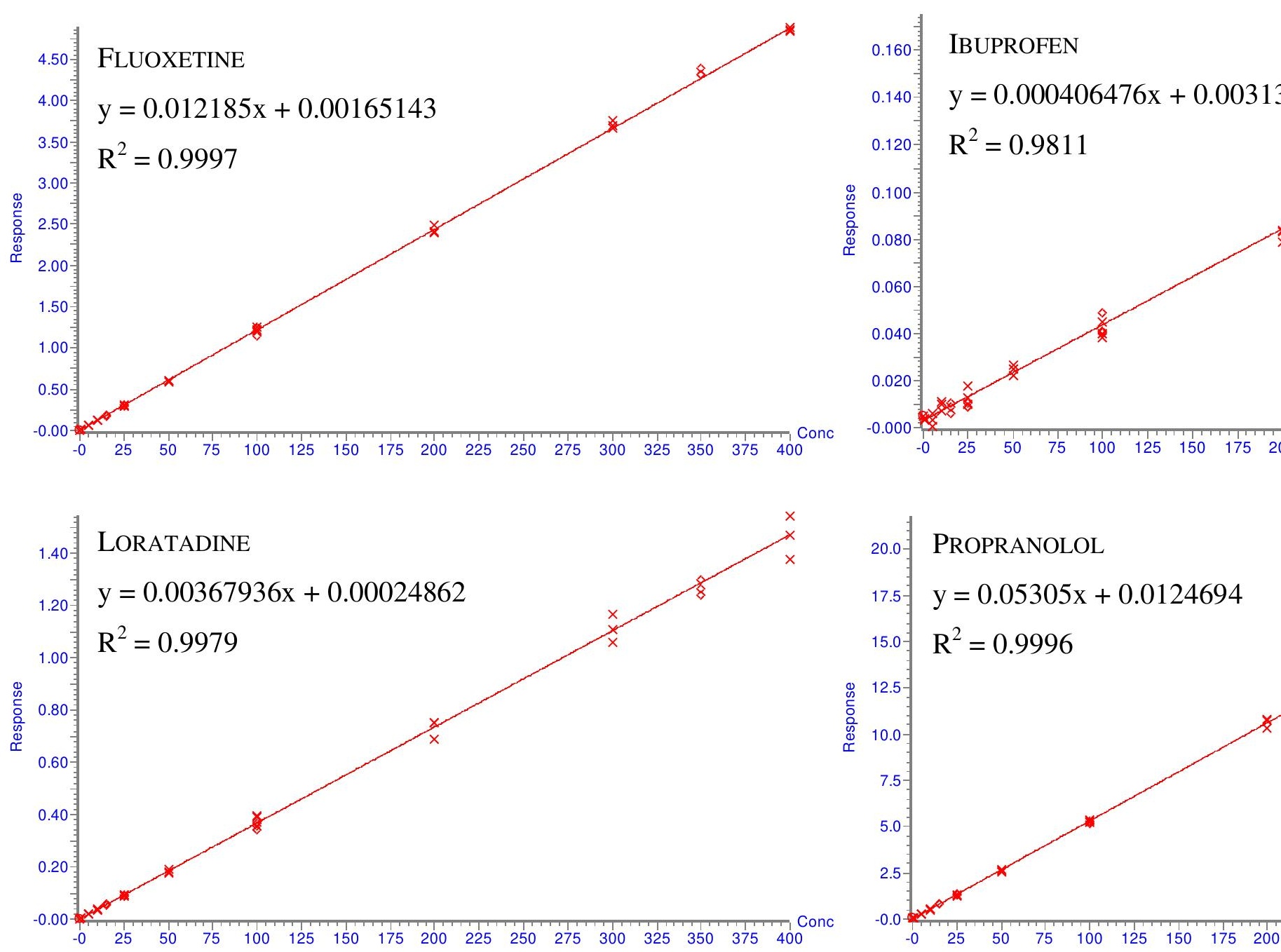


\section{Appendix 3.6}

Summary of the $1 / \mathrm{x}$ weighted quantitative data including linearity $\left(\mathrm{R}^{2}\right)$, instrument detection limit (IDL), instrument quantitation limit (IQL), mean percentage accuracy and precision of quality control sample (QC) concentration for each pharmaceutical analysed using the Xselect HSS T3 column.

\begin{tabular}{|c|c|c|c|c|c|c|c|c|c|c|c|}
\hline \multirow{3}{*}{ Pharmaceutical } & \multirow{3}{*}{$\begin{array}{c}\text { Linearity } \\
\left(\mathbf{R}^{2}\right)\end{array}$} & \multirow{3}{*}{$\begin{array}{c}\text { IDL } \\
(\mathbf{n g} / \mathbf{m L})\end{array}$} & \multirow{3}{*}{$\begin{array}{c}\text { IQL } \\
(\mathbf{n g} / \mathbf{m L})\end{array}$} & \multicolumn{8}{|c|}{ QCs } \\
\hline & & & & \multicolumn{4}{|c|}{ Mean Accuracy (\%) } & \multicolumn{4}{|c|}{ Mean Precision (\%) } \\
\hline & & & & QC1 & QC2 & QC3 & QC4 & QC1 & QC2 & QC3 & $\overline{Q C 4}$ \\
\hline Acetaminophen & 0.9950 & 5.87 & 19.58 & -12.59 & -7.86 & -1.51 & 5.87 & 9.48 & 3.54 & 2.75 & 4.51 \\
\hline Propranolol & 0.9996 & 0.78 & 2.61 & 1.85 & 0.49 & -1.63 & 0.38 & 1.75 & 1.78 & 1.51 & 1.19 \\
\hline Diphenhydramine & 0.9991 & 0.30 & 0.99 & 0.92 & 4.61 & 2.72 & 1.32 & 3.20 & 1.70 & 1.18 & 0.35 \\
\hline Citalopram & 0.9966 & 0.22 & 0.73 & 12.22 & 16.00 & 9.57 & -1.39 & 2.59 & 2.84 & 2.06 & 0.86 \\
\hline Erythromycin & 0.9996 & 0.55 & 1.82 & 0.22 & 0.24 & -1.34 & -0.85 & 3.48 & 6.23 & 2.88 & 2.23 \\
\hline Carbamazepine & 0.9988 & 0.51 & 1.69 & 7.30 & 7.06 & 3.94 & -1.92 & 1.80 & 2.45 & 1.61 & 1.00 \\
\hline Fluoxetine & 0.9997 & 0.14 & 0.47 & -2.40 & -1.10 & -0.66 & 1.65 & 3.28 & 4.49 & 4.50 & 1.13 \\
\hline Loratadine & 0.9979 & 0.47 & 1.57 & 0.31 & -2.99 & -3.34 & -1.44 & 2.15 & 2.56 & 3.02 & 2.28 \\
\hline Diclofenac & 0.9983 & 1.04 & 3.46 & -2.93 & 4.42 & 3.41 & 3.45 & 8.58 & 10.50 & 4.42 & 0.80 \\
\hline Ibuprofen & 0.9811 & 17.04 & 64.41 & -12.01 & -37.95 & -0.97 & 0.60 & 45.37 & 12.14 & 12.15 & 6.41 \\
\hline
\end{tabular}




\section{Appendix 3.7}

A summary of stability data, detailing the peak areas for each compound at each concentration and the calcu t0 and each data point used to assess compound stability as a mixture over time.

\begin{tabular}{|c|c|c|c|c|c|c|c|c|c|}
\hline \multirow[b]{2}{*}{ Compound } & \multirow[b]{2}{*}{$Q C$} & \multirow[b]{2}{*}{$\begin{array}{c}\text { Conc } \\
(n g / m L)\end{array}$} & \multicolumn{2}{|c|}{$\begin{array}{c}\text { Mean } \\
\text { Concentration of } \\
\text { Drug }\end{array}$} & \multirow{2}{*}{$\begin{array}{c}\% \text { Diff } \\
\text { between } \\
\text { t28 and } \\
\text { t0 }\end{array}$} & \multicolumn{2}{|c|}{ Peak Area for IS } & \multirow{2}{*}{$\begin{array}{c}\% \text { Diff } \\
\text { between } \\
\text { t28 and } \\
\text { t0 }\end{array}$} & \\
\hline & & & 0 & 28 & & 0 & 28 & & \\
\hline \multirow{4}{*}{$\begin{array}{l}\text { Acetaminophen } \\
\text { (IS: } \\
\text { Acetaminophen- } \\
\quad \text { (methyl)-d } \mathrm{d}_{3}\end{array}$} & Vlow & 15 & 13.63 & 13.39 & $-1.80 \%$ & 41870.08 & 41713.28 & $-0.37 \%$ & 0.5 \\
\hline & Low & 25 & 23.78 & 22.32 & $-6.14 \%$ & 40486.69 & 40798.40 & $0.77 \%$ & 1.2 \\
\hline & Mid & 100 & 101.69 & 97.41 & $-4.21 \%$ & 40900.50 & 40318.96 & $-1.42 \%$ & 1.3 \\
\hline & High & 350 & 373.13 & 349.15 & $-6.43 \%$ & 41133.97 & 41479.97 & $0.84 \%$ & 11. \\
\hline \multirow{4}{*}{$\begin{array}{l}\text { Propranolol (IS: } \\
\text { Pronethalol) }\end{array}$} & Vlow & 15 & 11.35 & 14.63 & $28.96 \%$ & 19978.07 & 17846.54 & $-10.67 \%$ & 0. \\
\hline & Low & 25 & 19.14 & 23.64 & $23.49 \%$ & 18864.60 & 18198.21 & $-3.53 \%$ & 0.3 \\
\hline & Mid & 100 & 77.87 & 98.45 & $26.42 \%$ & 18983.17 & 17669.82 & $-6.92 \%$ & 0.6 \\
\hline & High & 350 & 271.47 & 339.08 & $24.90 \%$ & 18797.19 & 17503.08 & $-6.88 \%$ & 1.4 \\
\hline \multirow{4}{*}{$\begin{array}{l}\text { Diphenhydramine } \\
\text { (IS: Talopram) }\end{array}$} & Vlow & 15 & 15.21 & 14.64 & $-3.79 \%$ & 84227.65 & 83972.36 & $-0.30 \%$ & 0.5 \\
\hline & Low & 25 & 26.52 & 24.15 & $-8.92 \%$ & 77260.56 & 84348.39 & $9.17 \%$ & 0.8 \\
\hline & Mid & 100 & 107.92 & 101.23 & $-6.20 \%$ & 80884.38 & 82499.21 & $2.00 \%$ & 2. \\
\hline & High & 350 & 368.14 & 343.26 & $-6.76 \%$ & 81117.51 & 83873.93 & $3.40 \%$ & 9.3 \\
\hline \multirow{4}{*}{$\begin{array}{l}\text { Citalopram (IS: } \\
\text { Talopram) }\end{array}$} & Vlow & 15 & 18.53 & 15.92 & $-14.08 \%$ & 84227.65 & 83972.36 & $-0.30 \%$ & 0.9 \\
\hline & Low & 25 & 31.89 & 26.90 & $-15.65 \%$ & 77260.56 & 84348.39 & $9.17 \%$ & 0.3 \\
\hline & Mid & 100 & 121.77 & 106.68 & $-12.39 \%$ & 80884.38 & 82499.21 & $2.00 \%$ & 0.9 \\
\hline & High & 350 & 387.99 & 332.01 & $-14.43 \%$ & 81117.51 & 83873.93 & $3.40 \%$ & 9.7 \\
\hline
\end{tabular}




\begin{tabular}{|c|c|c|c|c|c|c|c|c|c|}
\hline \multirow{4}{*}{$\begin{array}{c}\text { Erythromycin (IS: } \\
\text { 10,11-DHC) }\end{array}$} & Vlow & 15 & 14.77 & 16.12 & $9.13 \%$ & 175705.19 & 133721.23 & $-23.89 \%$ & \\
\hline & Low & 25 & 23.44 & 27.57 & $17.64 \%$ & 173365.62 & 130305.01 & $-24.84 \%$ & \\
\hline & Mid & 100 & 94.04 & 110.10 & $17.08 \%$ & 175577.59 & 131581.60 & $-25.06 \%$ & \\
\hline & High & 350 & 326.38 & 363.47 & $11.36 \%$ & 178604.02 & 134953.90 & $-24.44 \%$ & 19 \\
\hline \multirow{4}{*}{$\begin{array}{l}\text { Carbamazepine } \\
\text { (IS: } 10,11-\mathrm{DHC})\end{array}$} & Vlow & 15 & 15.82 & 15.51 & $-1.99 \%$ & 175705.19 & 133721.23 & $-23.89 \%$ & \\
\hline & Low & 25 & 25.76 & 25.89 & $0.49 \%$ & 173365.62 & 130305.01 & $-24.84 \%$ & \\
\hline & Mid & 100 & 103.97 & 103.06 & $-0.88 \%$ & 175577.59 & 131581.60 & $-25.06 \%$ & \\
\hline & High & 350 & 343.56 & 348.96 & $1.57 \%$ & 178604.02 & 134953.90 & $-24.44 \%$ & \\
\hline \multirow{4}{*}{$\begin{array}{l}\text { Fluoxetine (IS: } \\
\text { Talopram) }\end{array}$} & Vlow & 15 & 19.30 & 16.55 & $-14.28 \%$ & 84227.65 & 83972.36 & $-0.30 \%$ & \\
\hline & Low & 25 & 28.82 & 26.50 & $-8.05 \%$ & 77260.56 & 84348.39 & $9.17 \%$ & \\
\hline & Mid & 100 & 117.71 & 102.73 & $-12.73 \%$ & 80884.38 & 82499.21 & $2.00 \%$ & \\
\hline & High & 350 & 443.86 & 392.98 & $-11.46 \%$ & 81117.51 & 83873.93 & $3.40 \%$ & \\
\hline \multirow{4}{*}{$\begin{array}{c}\text { Loratadine (IS: } \\
\text { 10,11-DHC) }\end{array}$} & Vlow & 15 & 18.93 & 14.74 & $-22.14 \%$ & 175705.19 & 133721.23 & $-23.89 \%$ & \\
\hline & Low & 25 & 31.30 & 24.31 & $-22.31 \%$ & 173365.62 & 130305.01 & $-24.84 \%$ & \\
\hline & Mid & 100 & 127.58 & 99.24 & $-22.21 \%$ & 175577.59 & 131581.60 & $-25.06 \%$ & \\
\hline & High & 350 & 432.83 & 346.47 & $-19.95 \%$ & 178604.02 & 134953.90 & $-24.44 \%$ & \\
\hline \multirow{4}{*}{$\begin{array}{c}\text { Diclofenac (IS: } \\
\text { Talopram) }\end{array}$} & Vlow & 15 & 15.66 & 14.84 & $-5.22 \%$ & 84227.65 & 83972.36 & $-0.30 \%$ & \\
\hline & Low & 25 & 28.26 & 25.20 & $-10.80 \%$ & 77260.56 & 84348.39 & $9.17 \%$ & \\
\hline & Mid & 100 & 96.20 & 98.29 & $2.18 \%$ & 80884.38 & 82499.21 & $2.00 \%$ & \\
\hline & High & 350 & 323.99 & 334.93 & $3.38 \%$ & 81117.51 & 83873.93 & $3.40 \%$ & \\
\hline \multirow{4}{*}{$\begin{array}{l}\text { Ibuprofen (IS: } \\
\text { Pronethalol) }\end{array}$} & Vlow & 15 & 7.70 & 16.60 & $115.67 \%$ & 19978.07 & 17846.54 & $-10.67 \%$ & \\
\hline & Low & 25 & 19.13 & 21.08 & $10.17 \%$ & 18864.60 & 18198.21 & $-3.53 \%$ & \\
\hline & Mid & 100 & 97.49 & 88.61 & $-9.10 \%$ & 18983.17 & 17669.82 & $-6.92 \%$ & \\
\hline & High & 350 & 393.38 & 355.32 & $-9.67 \%$ & 18797.19 & 17503.08 & $-6.88 \%$ & 32 \\
\hline
\end{tabular}




\section{Appendix 4.1}

Mass spectra obtained for each of the biocides studied. Inset: compounds information, including name, product ion and optimum collision energy (CE) used to induce fragmentation.

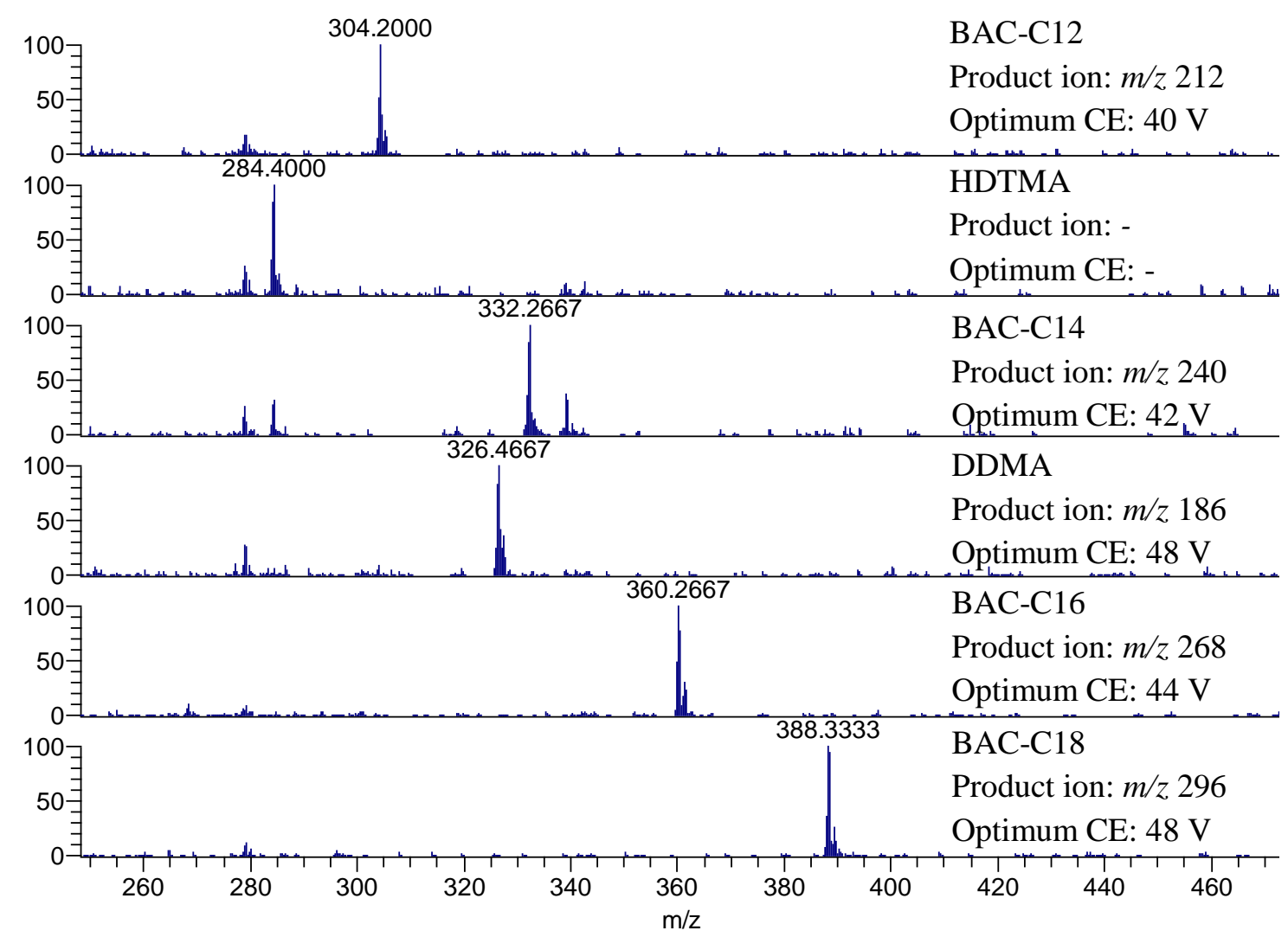




\section{Appendix 4.2}

Data used to compare linear and $1 / \mathrm{x}$ weighted regression functions, including the linearity, instrument detection limit (IDL), mean accuracies and mean precision values for each biocide.

\section{Linear}

\begin{tabular}{|c|c|c|c|c|c|c|c|c|c|c|}
\hline \multirow{3}{*}{ Biocide } & \multirow{3}{*}{$\begin{array}{c}\text { Linearity } \\
\qquad\left(\mathbf{R}^{2}\right)\end{array}$} & \multirow{3}{*}{$\begin{array}{c}\text { IDL } \\
(\mathrm{ng} / \mathrm{mL})\end{array}$} & \multicolumn{8}{|c|}{ QCs } \\
\hline & & & \multicolumn{4}{|c|}{ Mean Accuracy (\%) } & \multicolumn{4}{|c|}{ Mean Precision (\%) } \\
\hline & & & QC1 & QC2 & QC3 & QC4 & QC1 & QC2 & QC3 & QC4 \\
\hline BAC-C12 & .9843 & $0.30 \pm 0.1$ & 3.74 & -7.48 & -11.11 & -4.63 & 13.90 & 16.31 & 17.62 & 9.47 \\
\hline BAC-C14 & 0.9854 & $0.27 \pm 0.1$ & 8.85 & -1.97 & 1.12 & -4.64 & 9.76 & 15.96 & 20.09 & 13.27 \\
\hline BAC-C16 & 0.9694 & $0.19 \pm 0.1$ & 17.99 & -12.42 & -3.67 & -9.86 & 17.25 & 22.60 & 13.71 & 5.09 \\
\hline BAC-C18 & 0.9604 & $0.76 \pm 0.3$ & 29.04 & -13.99 & -0.48 & -6.86 & 16.58 & 19.12 & 15.05 & 1.43 \\
\hline DDMA & 0.9794 & $0.99 \pm 0.3$ & 18.47 & -6.68 & -2.50 & -8.23 & 8.12 & 24.75 & 10.33 & 10.76 \\
\hline HDTMA & 0.9729 & $1.79 \pm 0.6$ & 26.67 & -5.43 & 0.50 & -4.29 & 7.30 & 13.60 & 10.95 & 7.23 \\
\hline
\end{tabular}

$1 / \mathbf{x}$

\begin{tabular}{|c|c|c|c|c|c|c|c|c|c|c|}
\hline \multirow{3}{*}{ Biocide } & \multirow{3}{*}{$\begin{array}{c}\text { Linearity } \\
\left(\mathbf{R}^{2}\right)\end{array}$} & \multirow{3}{*}{$\begin{array}{c}\text { IDL } \\
(\mathrm{ng} / \mathrm{mL})\end{array}$} & \multicolumn{8}{|c|}{ QCs } \\
\hline & & & \multicolumn{4}{|c|}{ Mean Accuracy (\%) } & \multicolumn{4}{|c|}{ Mean Precision (\%) } \\
\hline & & & QC1 & QC2 & QC3 & QC4 & QC1 & QC2 & QC3 & QC4 \\
\hline BAC-C12 & 9729 & $0.32 \pm 0.1$ & -12.80 & -15.24 & -5.56 & -1.82 & 17.40 & 18.00 & 18.17 & 9.68 \\
\hline BAC-C14 & 0.9891 & $0.28 \pm 0.1$ & -1.28 & -1.05 & -0.59 & -2.90 & 11.12 & 16.85 & 20.45 & 13.46 \\
\hline BAC-C16 & 0.9759 & $0.20 \pm 0.1$ & 2.95 & -7.20 & -10.83 & -7.45 & 20.76 & 24.64 & 14.14 & 5.21 \\
\hline BAC-C18 & 0.9669 & $0.82 \pm 0.3$ & 5.04 & -6.11 & -11.49 & -2.61 & 22.06 & 21.94 & 15.83 & 1.48 \\
\hline DDMA & 0.9806 & $1.03 \pm 0.3$ & 6.54 & -5.28 & -5.20 & -6.25 & 9.39 & 26.48 & 10.58 & 10.95 \\
\hline HDTMA & 0.9764 & $1.87 \pm 0.6$ & 13.38 & -2.56 & -3.67 & -1.84 & 8.53 & 14.66 & 11.24 & 7.37 \\
\hline
\end{tabular}




\section{Appendix 4.3}

Calibration graphs for each compound in the suite, generated using QuanBrowser 2.0.1 software. These graphs show the linear regression statistics for the Xselect HSS T3 column.
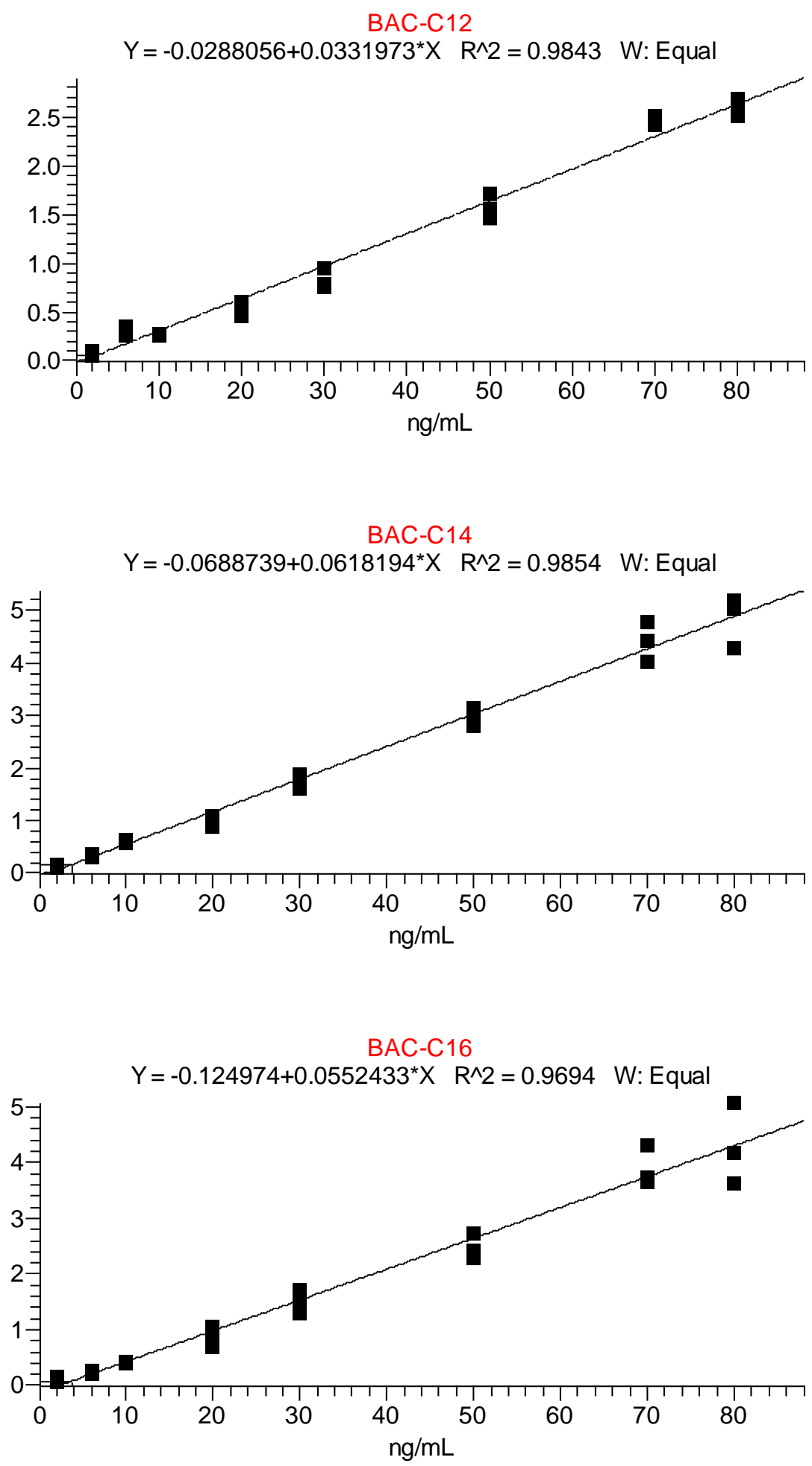
$\mathrm{BAC}-\mathrm{C} 18$

$Y=-0.141772+0.0440929^{\star} X \quad R^{\wedge} 2=0.9604 \quad W:$ Equal

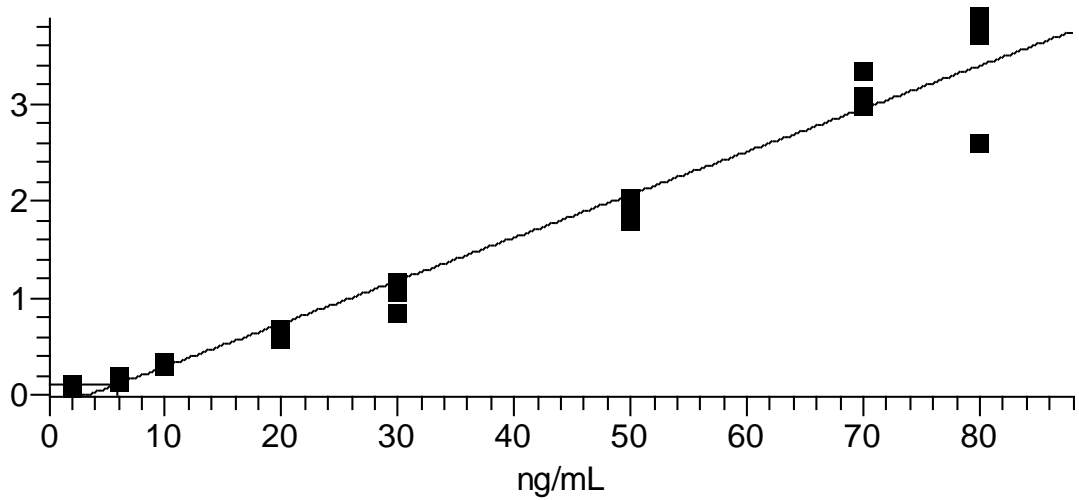

DDMA

$Y=-0.0255394+0.0249155^{\star} X \quad R^{\wedge} 2=0.9794 \quad W:$ Equal

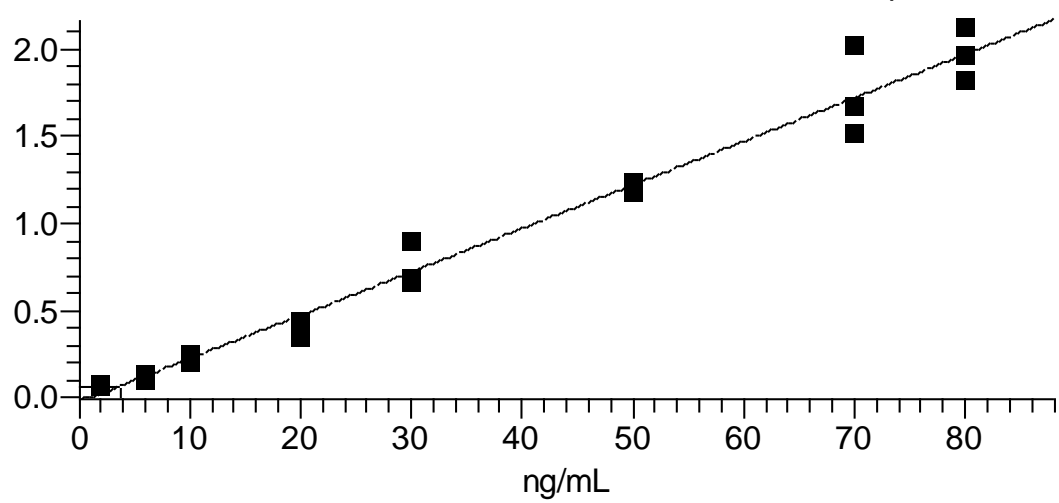

HDTMA

$Y=-0.0497472+0.0548498^{*} X \quad R^{\wedge} 2=0.9729 \quad W:$ Equal

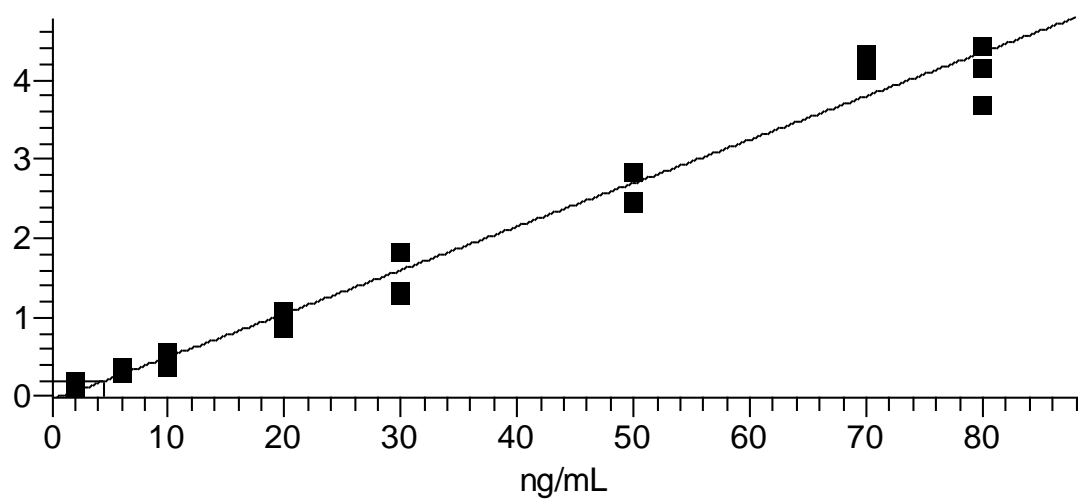




\section{Appendix 4.4}

The table shows the intra- and inter-day precision values determined for each biocide using the LCQ analytical method. Inter-day precision was determined over three data sets.

\begin{tabular}{|c|c|c|c|c|c|}
\hline \multirow[b]{2}{*}{ Compound } & \multirow[b]{2}{*}{$\begin{array}{c}\text { Precision } \\
\text { (\% } \% \text { RSD) }\end{array}$} & \multicolumn{4}{|c|}{$Q C s$} \\
\hline & & $\begin{array}{c}\text { QC1 } \\
(8 \mathrm{ng} / \mathrm{mL})\end{array}$ & $\begin{array}{c}\mathrm{QC2} \\
(20 \mathrm{ng} / \mathrm{mL})\end{array}$ & $\begin{array}{c}\text { QC3 } \\
(60 \mathrm{ng} / \mathrm{mL})\end{array}$ & $\begin{array}{c}\text { QC4 } \\
(80 \mathrm{ng} / \mathrm{mL})\end{array}$ \\
\hline \multirow{2}{*}{ BAC-C12 } & Intra-Day & 13.90 & 16.31 & 17.62 & 9.47 \\
\hline & Inter-Day & 12.55 & 17.67 & 19.10 & 10.83 \\
\hline \multirow{2}{*}{ BAC-C14 } & Intra-Day & 9.76 & 15.96 & 20.08 & 13.27 \\
\hline & Inter-Day & 9.26 & 12.02 & 17.19 & 13.60 \\
\hline \multirow{2}{*}{ BAC-C16 } & Intra-Day & 17.25 & 22.60 & 13.71 & 5.09 \\
\hline & Inter-Day & 11.83 & 11.38 & 14.49 & 8.95 \\
\hline \multirow{2}{*}{ BAC-C18 } & Intra-Day & 16.58 & 19.12 & 15.05 & 1.43 \\
\hline & Inter-Day & 14.77 & 15.40 & 19.21 & 13.11 \\
\hline \multirow{2}{*}{ DDMA } & Intra-Day & 8.12 & 24.75 & 10.33 & 10.76 \\
\hline & Inter-Day & 19.08 & 17.27 & 18.34 & 13.39 \\
\hline \multirow{2}{*}{ HDTMA } & Intra-Day & 7.30 & 13.60 & 10.95 & 7.23 \\
\hline & Inter-Day & 11.84 & 12.24 & 14.49 & 9.19 \\
\hline
\end{tabular}




\section{Appendix 5.1}

Summary of the calculated matrix effects (\%ME) and recovery (\%REC) and associated precision of measurements (\%RSD) of each of the pharmaceuticals studied when extracted using standardised unbuffered QuEChERS methods.

\begin{tabular}{|c|c|c|c|c|c|}
\hline Compound & $\begin{array}{c}\text { Method } \\
\text { (unbuffered) }\end{array}$ & $\% \mathrm{ME}$ & \% RSD & $\%$ REC & $\%$ RSD \\
\hline \multirow{2}{*}{$\begin{array}{l}\text { Acetaminophen- } \\
\text { (methyl)- } \mathrm{d}_{3}\end{array}$} & EN Method with PSA dSPE & 134.11 & 7.07 & 71.53 & 2.03 \\
\hline & AOAC Method with PSA dSPE & 137.67 & 36.01 & 37.66 & 16.25 \\
\hline \multirow{2}{*}{ Acetaminophen } & EN Method with PSA dSPE & 114.24 & 3.94 & 53.23 & 7.21 \\
\hline & AOAC Method with PSA dSPE & 119.49 & 30.06 & 25.37 & 11.80 \\
\hline \multirow{2}{*}{ Pronethalol } & EN Method with PSA dSPE & 141.77 & 3.67 & 41.62 & 0.65 \\
\hline & AOAC Method with PSA dSPE & 101.05 & 0.47 & 12.01 & 21.77 \\
\hline \multirow{2}{*}{ Propranolol } & EN Method with PSA dSPE & 286.59 & 2.40 & 43.05 & 1.44 \\
\hline & AOAC Method with PSA dSPE & 103.16 & 7.71 & 11.34 & 10.51 \\
\hline \multirow{2}{*}{ Diphenhydramine } & EN Method with PSA dSPE & 256.87 & 5.45 & 38.21 & 13.20 \\
\hline & AOAC Method with PSA dSPE & 119.42 & 24.08 & 10.78 & 6.05 \\
\hline \multirow{2}{*}{ Citalopram } & EN Method with PSA dSPE & 255.37 & 1.55 & 43.91 & 3.56 \\
\hline & AOAC Method with PSA dSPE & 116.09 & 20.00 & 12.58 & 9.89 \\
\hline \multirow{2}{*}{ Erythromycin } & EN Method with PSA dSPE & 308.95 & 6.42 & 40.2 & 1.67 \\
\hline & AOAC Method with PSA dSPE & 103.52 & 9.35 & 12.91 & 6.71 \\
\hline \multirow{2}{*}{ Carbamazepine } & EN Method with PSA dSPE & 101.10 & 0.88 & 48.25 & 1.82 \\
\hline & AOAC Method with PSA dSPE & 99.72 & 0.71 & 24.05 & 17.05 \\
\hline \multirow{2}{*}{$\begin{array}{l}10,11- \\
\text { Dihydrocarbamazepine }\end{array}$} & EN Method with PSA dSPE & 103.91 & 1.79 & 46.54 & 0.35 \\
\hline & AOAC Method with PSA dSPE & 101.50 & 2.06 & 23.33 & 14.56 \\
\hline \multirow{2}{*}{ Fluoxetine } & EN Method with PSA dSPE & 159.18 & 4.79 & 43.90 & 2.42 \\
\hline & AOAC Method with PSA dSPE & 122.83 & 27.15 & 7.70 & 13.55 \\
\hline \multirow{2}{*}{ Talopram } & EN Method with PSA dSPE & 210.10 & 4.17 & 42.23 & 0.08 \\
\hline & AOAC Method with PSA dSPE & 127.72 & 32.81 & 5.15 & 18.5 \\
\hline \multirow{2}{*}{ Loratadine } & EN Method with PSA dSPE & 100.22 & 2.41 & 44.9 & 4.88 \\
\hline & AOAC Method with PSA dSPE & 96.53 & 1.74 & 22.43 & 15.36 \\
\hline \multirow{2}{*}{ Diclofenac } & EN Method with PSA dSPE & 97.74 & 3.12 & 10.96 & 16.95 \\
\hline & AOAC Method with PSA dSPE & 96.01 & 1.34 & 8.89 & 5.81 \\
\hline \multirow{2}{*}{ Ibuprofen } & EN Method with PSA dSPE & 109.8 & 12.84 & 9.48 & 33.50 \\
\hline & AOAC Method with PSA dSPE & 108.34 & 24.3 & 3.26 & 53.34 \\
\hline
\end{tabular}




\section{Appendix 5.2}

Summary of the calculated matrix effects (\%ME) and recovery (\%REC) and associated precision of measurements (\%RSD) of each of the pharmaceuticals studied when extracted using modified QuEChERS methods during method development.

\begin{tabular}{|c|c|c|c|c|c|}
\hline Compound & $\begin{array}{c}\text { Method } \\
\text { (custom extraction) }\end{array}$ & $\% \mathrm{ME}$ & $\%$ RSD & \% REC & $\%$ RSD \\
\hline \multirow{3}{*}{$\begin{array}{l}\text { Acetaminophen- } \\
(\text { methyl })-\mathrm{d}_{3}\end{array}$} & Method A & 121.62 & 8.84 & 84.42 & 2.42 \\
\hline & Method B & 74.94 & 2.03 & 81.94 & 4.49 \\
\hline & Method C & 76.78 & 4.77 & 90.05 & 2.61 \\
\hline \multirow{3}{*}{ Acetaminophen } & Method A & 64.86 & 5.68 & 62.88 & 14.20 \\
\hline & Method B & 57.45 & 6.31 & 79.85 & 17.75 \\
\hline & Method C & 63.36 & 5.73 & 88.23 & 4.54 \\
\hline \multirow{3}{*}{ Pronethalol } & Method A & 35.72 & 6.25 & 43.61 & 4.27 \\
\hline & Method B & 39.43 & 4.91 & 51.05 & 20.49 \\
\hline & Method C & 43.15 & 6.77 & 82.74 & 18.41 \\
\hline \multirow{3}{*}{ Propranolol } & Method A & 42.30 & 4.53 & 40.65 & 12.37 \\
\hline & Method B & 41.65 & 4.61 & 52.48 & 16.17 \\
\hline & Method C & 49.66 & 2.03 & 91.62 & 21.50 \\
\hline \multirow{3}{*}{ Diphenhydramine } & Method A & 47.76 & 0.76 & 43.04 & 10.59 \\
\hline & Method B & 47.22 & 11.32 & 58.26 & 17.82 \\
\hline & Method C & 60.71 & 5.51 & 92.37 & 25.88 \\
\hline \multirow{3}{*}{ Citalopram } & Method A & 46.03 & 4.86 & 42.39 & 12.26 \\
\hline & Method B & 45.58 & 9.03 & 56.91 & 18.85 \\
\hline & Method C & 58.74 & 2.23 & 91.05 & 25.91 \\
\hline \multirow{3}{*}{ Erythromycin } & Method A & 44.59 & 10.37 & 35.73 & 5.66 \\
\hline & Method B & 42.02 & 4.85 & 41.71 & 4.36 \\
\hline & Method C & 49.18 & 6.83 & 94.00 & 19.10 \\
\hline \multirow{3}{*}{ Carbamazepine } & Method A & 43.82 & 4.54 & 46.75 & 21.06 \\
\hline & Method B & 43.79 & 2.14 & 55.17 & 2.03 \\
\hline & Method C & 44.57 & 4.95 & 81.33 & 4.16 \\
\hline
\end{tabular}




\begin{tabular}{|c|c|c|c|c|c|}
\hline \multirow{3}{*}{$\begin{array}{l}\text { 10,11- } \\
\text { Dihydrocarbamazepine }\end{array}$} & Method A & 42.92 & 0.60 & 47.09 & 7.18 \\
\hline & Method B & 41.5 & 2.35 & 56.7 & 3.14 \\
\hline & Method C & 44.23 & 1.90 & 81.75 & 3.06 \\
\hline \multirow{3}{*}{ Fluoxetine } & Method A & 58.91 & 3.94 & 41.47 & 10.46 \\
\hline & Method B & 51.66 & 13.32 & 52.11 & 11.23 \\
\hline & Method C & 52.78 & 16.13 & 76.90 & 3.42 \\
\hline \multirow{3}{*}{ Talopram } & Method A & 51.86 & 3.34 & 41.32 & 12.14 \\
\hline & Method B & 50.45 & 12.72 & 56.53 & 19.84 \\
\hline & Method C & 73.05 & 4.16 & 75.52 & 10.19 \\
\hline \multirow{3}{*}{ Loratadine } & Method A & 40.71 & 5.53 & 50.71 & 5.46 \\
\hline & Method B & 43.84 & 3.32 & 56.20 & 4.46 \\
\hline & Method C & 44.78 & 1.65 & 81.01 & 2.63 \\
\hline \multirow{3}{*}{ Diclofenac } & Method A & 40.74 & 0.74 & 3.99 & 45.15 \\
\hline & Method B & 44.02 & 7.47 & 19.85 & 49.55 \\
\hline & Method C & 43.51 & 4.60 & 6.17 & 37.78 \\
\hline \multirow{3}{*}{ Ibuprofen } & Method A & 42.46 & 13.37 & 28.36 & 13.71 \\
\hline & Method B & 42.49 & 21.97 & 30.13 & 35.49 \\
\hline & Method C & 39.47 & 5.33 & 2.45 & 50.48 \\
\hline
\end{tabular}




\section{Appendix 5.3}

Summary of the calculated matrix effects (\%ME) and recovery (\%REC) and associated precision of measurements (\%RSD) of each of the pharmaceuticals studied when extracted using alternative QuEChERS dSPE sorbents during method development.

\begin{tabular}{|c|c|c|c|c|c|}
\hline Compound & $\begin{array}{c}\text { Method } \\
\text { (custom extraction) }\end{array}$ & $\% \mathrm{ME}$ & $\%$ RSD & \% REC & $\%$ RSD \\
\hline \multirow{3}{*}{$\begin{array}{l}\text { Acetaminophen- } \\
\text { (methyl)- } \mathrm{d}_{3}\end{array}$} & EN F\&V dSPE & 105.17 & 1.05 & 115.30 & 19.85 \\
\hline & EN Waxed dSPE & 201.63 & 28.19 & 84.41 & 23.93 \\
\hline & EN Pigmented dSPE & 284.09 & 6.37 & 102.59 & 8.07 \\
\hline \multirow{3}{*}{ Acetaminophen } & EN F\&V dSPE & 59.73 & 6.60 & 90.88 & 10.11 \\
\hline & EN Waxed dSPE & \multicolumn{4}{|c|}{ Not able to integrate - split chromatography } \\
\hline & EN Pigmented dSPE & 77.09 & 7.23 & 74.06 & 16.63 \\
\hline \multirow{3}{*}{ Pronethalol } & EN F\&V dSPE & 40.40 & 4.94 & 59.43 & 17.80 \\
\hline & EN Waxed dSPE & 46.36 & 6.50 & 20.64 & 18.24 \\
\hline & EN Pigmented dSPE & 43.90 & 5.35 & 48.50 & 5.71 \\
\hline \multirow{3}{*}{ Propranolol } & EN F\&V dSPE & 61.53 & 4.92 & 69.07 & 40.28 \\
\hline & EN Waxed dSPE & 67.67 & 8.51 & 17.02 & 37.34 \\
\hline & EN Pigmented dSPE & 63.37 & 12.3 & 42.30 & 7.83 \\
\hline \multirow{3}{*}{ Diphenhydramine } & EN F\&V dSPE & 71.52 & 6.53 & 37.71 & 13.08 \\
\hline & EN Waxed dSPE & 78.42 & 2.20 & 18.50 & 23.46 \\
\hline & EN Pigmented dSPE & 71.13 & 17.72 & 32.09 & 8.59 \\
\hline \multirow{3}{*}{ Citalopram } & EN F\&V dSPE & 89.36 & 0.46 & 56.76 & 13.05 \\
\hline & EN Waxed dSPE & 79.17 & 2.95 & 33.99 & 22.64 \\
\hline & EN Pigmented dSPE & 90.30 & 12.89 & 59.14 & 8.04 \\
\hline \multirow{3}{*}{ Erythromycin } & EN F\&V dSPE & 57.85 & 3.38 & 32.19 & 19.98 \\
\hline & EN Waxed dSPE & 62.13 & 12.48 & 23.08 & 39.58 \\
\hline & EN Pigmented dSPE & 59.82 & 17.28 & 28.44 & 13.29 \\
\hline \multirow{3}{*}{ Carbamazepine } & EN F\&V dSPE & 53.21 & 8.33 & 71.68 & 0.27 \\
\hline & EN Waxed dSPE & 42.71 & 4.68 & 65.94 & 10.34 \\
\hline & EN Pigmented dSPE & 43.80 & 5.84 & 68.43 & 3.69 \\
\hline
\end{tabular}




\begin{tabular}{|c|c|c|c|c|c|}
\hline \multirow{3}{*}{$\begin{array}{l}\text { 10,11- } \\
\text { Dihydrocarbamazepine }\end{array}$} & EN F\&V dSPE & 42.03 & 4.35 & 67.59 & 3.81 \\
\hline & EN Waxed dSPE & 39.65 & 4.59 & 61.71 & 3.22 \\
\hline & EN Pigmented dSPE & 40.33 & 0.94 & 68.16 & 4.44 \\
\hline \multirow{3}{*}{ Fluoxetine } & EN F\&V dSPE & 41.07 & 0.24 & 44.48 & 3.40 \\
\hline & EN Waxed dSPE & 52.08 & 5.03 & 19.44 & 14.25 \\
\hline & EN Pigmented dSPE & 55.74 & 4.00 & 44.31 & 4.17 \\
\hline \multirow{3}{*}{ Talopram } & EN F\&V dSPE & 69.35 & 4.87 & 51.18 & 6.37 \\
\hline & EN Waxed dSPE & 67.53 & 8.57 & 18.86 & 11.08 \\
\hline & EN Pigmented dSPE & 73.91 & 5.71 & 51.34 & 3.32 \\
\hline \multirow{3}{*}{ Loratadine } & EN F\&V dSPE & 43.32 & 2.00 & 64.54 & 0.40 \\
\hline & EN Waxed dSPE & 42.72 & 5.39 & 60.23 & 4.33 \\
\hline & EN Pigmented dSPE & 45.34 & 4.69 & 66.88 & 2.83 \\
\hline \multirow{3}{*}{ Diclofenac } & EN F\&V dSPE & 40.36 & 2.04 & 0.80 & 141.42 \\
\hline & EN Waxed dSPE & 44.21 & 4.54 & 1.28 & 109.58 \\
\hline & EN Pigmented dSPE & 47.63 & 6.48 & 1.18 & 134.15 \\
\hline \multirow{3}{*}{ Ibuprofen } & EN F\&V dSPE & 44.82 & 11.29 & 43.53 & 14.37 \\
\hline & EN Waxed dSPE & 48.61 & 11.49 & 15.79 & 134.25 \\
\hline & EN Pigmented dSPE & 46.59 & 14.60 & 27.80 & 10.32 \\
\hline
\end{tabular}




\section{Appendix 5.4}

Summary of the calculated matrix effects (\%ME) and recovery (\%REC) and associated precision of measurements (\%RSD) of each of the pharmaceuticals studied to compare the difference between a QuEChERS extraction using a dSPE step and the equivalent sorbent type in a typical SPE cartridge form.

\begin{tabular}{|c|c|c|c|c|c|}
\hline Compound & $\begin{array}{c}\text { Method } \\
\text { (custom extraction) }\end{array}$ & $\% \mathrm{ME}$ & $\%$ RSD & $\%$ REC & $\%$ RSD \\
\hline \multirow{3}{*}{$\begin{array}{l}\text { Acetaminophen- } \\
(\text { methyl })-\mathrm{d}_{3}\end{array}$} & PSA dSPE & 164.03 & 4.28 & 84.46 & 9.91 \\
\hline & PSA Cartridge-SPE $-6 \mathrm{~mL}$ & 141.03 & 5.07 & 49.80 & 19.49 \\
\hline & PSA Cartridge-SPE - $15 \mathrm{~mL}$ & 149.50 & 4.45 & 71.51 & 14.11 \\
\hline \multirow{3}{*}{ Acetaminophen } & PSA dSPE & 96.35 & 3.43 & 92.13 & 20.87 \\
\hline & PSA Cartridge-SPE $-6 \mathrm{~mL}$ & 99.71 & 11.67 & 49.88 & 26.41 \\
\hline & PSA Cartridge-SPE - $15 \mathrm{~mL}$ & 94.94 & 2.74 & 70.54 & 3.83 \\
\hline \multirow{3}{*}{ Pronethalol } & PSA dSPE & 97.16 & 1.10 & 44.5 & 9.36 \\
\hline & PSA Cartridge-SPE $-6 \mathrm{~mL}$ & 89.87 & 8.98 & 31.83 & 33.53 \\
\hline & PSA Cartridge-SPE - $15 \mathrm{~mL}$ & 85.67 & 3.54 & 36.08 & 28.57 \\
\hline \multirow{3}{*}{ Propranolol } & PSA dSPE & 118.61 & 10.11 & 44.04 & 9.81 \\
\hline & PSA Cartridge-SPE $-6 \mathrm{~mL}$ & 119.94 & 6.51 & 39.65 & 71.35 \\
\hline & PSA Cartridge-SPE - $15 \mathrm{~mL}$ & 108.89 & 0.78 & 34.06 & 19.31 \\
\hline \multirow{3}{*}{ Diphenhydramine } & PSA dSPE & 110.2 & 6.98 & 47.62 & 10.30 \\
\hline & PSA Cartridge-SPE - $6 \mathrm{~mL}$ & 164.11 & 9.48 & 42.04 & 44.84 \\
\hline & PSA Cartridge-SPE - $15 \mathrm{~mL}$ & 137.60 & 9.21 & 52.49 & 30.68 \\
\hline \multirow{3}{*}{ Citalopram } & PSA dSPE & 109.88 & 2.12 & 47.73 & 11.94 \\
\hline & PSA Cartridge-SPE $-6 \mathrm{~mL}$ & 150.71 & 27.80 & 43.25 & 69.81 \\
\hline & PSA Cartridge-SPE - $15 \mathrm{~mL}$ & 114.95 & 4.51 & 47.53 & 25.74 \\
\hline \multirow{3}{*}{ Erythromycin } & PSA dSPE & 112.36 & 3.95 & 43.83 & 12.71 \\
\hline & PSA Cartridge-SPE - $6 \mathrm{~mL}$ & 127.98 & 10.01 & 20.04 & 56.08 \\
\hline & PSA Cartridge-SPE - $15 \mathrm{~mL}$ & 109.98 & 1.30 & 45.53 & 16.44 \\
\hline \multirow{2}{*}{ Carbamazepine } & PSA dSPE & 101.19 & 5.28 & 55.4 & 12.7 \\
\hline & PSA Cartridge-SPE - $6 \mathrm{~mL}$ & 132.46 & 2.03 & 69.44 & 2.18 \\
\hline
\end{tabular}




\begin{tabular}{|c|c|c|c|c|c|}
\hline & PSA Cartridge-SPE - $15 \mathrm{~mL}$ & 114.39 & 2.64 & 72.47 & 4.18 \\
\hline \multirow{3}{*}{$\begin{array}{l}\text { 10,11- } \\
\text { Dihydrocarbamazepine }\end{array}$} & PSA dSPE & 100.45 & 3.62 & 53.36 & 10.57 \\
\hline & PSA Cartridge-SPE - $6 \mathrm{~mL}$ & 103.16 & 1.11 & 59.83 & 5.75 \\
\hline & PSA Cartridge-SPE - $15 \mathrm{~mL}$ & 97.57 & 3.09 & 62.4 & 2.88 \\
\hline \multirow{3}{*}{ Fluoxetine } & EN F\&V dSPE & 95.26 & 1.71 & 46.59 & 14.27 \\
\hline & EN Waxed dSPE & 97.55 & 2.50 & 25.27 & 21.84 \\
\hline & EN Pigmented dSPE & 91.70 & 0.97 & 38.08 & 16.96 \\
\hline \multirow{3}{*}{ Talopram } & PSA dSPE & 125.32 & 13.36 & 42.76 & 13.84 \\
\hline & PSA Cartridge-SPE $-6 \mathrm{~mL}$ & 153.62 & 1.69 & 17.28 & 22.54 \\
\hline & PSA Cartridge-SPE - $15 \mathrm{~mL}$ & 143.81 & 5.14 & 34.98 & 19.71 \\
\hline \multirow{3}{*}{ Loratadine } & PSA dSPE & 102.65 & 2.49 & 53.25 & 14.29 \\
\hline & PSA Cartridge-SPE $-6 \mathrm{~mL}$ & 103.16 & 2.93 & 55.66 & 10.89 \\
\hline & PSA Cartridge-SPE - $15 \mathrm{~mL}$ & 95.12 & 1.17 & 61.98 & 4.74 \\
\hline \multirow{3}{*}{ Diclofenac } & PSA dSPE & 107.19 & 3.38 & 1.10 & 124.92 \\
\hline & PSA Cartridge-SPE $-6 \mathrm{~mL}$ & 104.03 & 4.29 & 0.24 & 34.64 \\
\hline & PSA Cartridge-SPE - $15 \mathrm{~mL}$ & 102.24 & 1.45 & 0.21 & 68.33 \\
\hline \multirow{3}{*}{ Ibuprofen } & PSA dSPE & 105.77 & 16.49 & 12.36 & 55.44 \\
\hline & PSA Cartridge-SPE $-6 \mathrm{~mL}$ & 120.19 & 8.25 & 4.01 & 91.85 \\
\hline & PSA Cartridge-SPE - $15 \mathrm{~mL}$ & 119.08 & 3.88 & 3.51 & 101.07 \\
\hline
\end{tabular}




\section{Appendix 5.5}

A summary of the QuEChERS data obtained for the extractions of pharmaceuticals, and pharmaceutica percentage recoveries, matrix effects and relative standard deviation (\%RSD) for each extraction.

\begin{tabular}{|c|c|c|c|c|c|c|c|c|c|}
\hline \multirow[b]{3}{*}{ Compounds } & \multicolumn{6}{|c|}{ 20th June 2017 - Soil - Pharms Only } & \multirow{2}{*}{\multicolumn{3}{|c|}{$\begin{array}{c}\text { 1st August } 2017 \text { - Soil } \\
\text { Recovery }\end{array}$}} \\
\hline & \multicolumn{3}{|c|}{ Recovery } & \multicolumn{3}{|c|}{ Matrix Effects } & & & \\
\hline & Mean RE & St Dev & $\%$ RSD & Mean ME & St Dev & \% RSD & Mean RE & St Dev & \\
\hline Acet-d $\mathrm{d}_{3}$ & $75.86 \%$ & 0.04 & 5.25 & $150.34 \%$ & 0.06 & 4.27 & $100.12 \%$ & 0.12 & \\
\hline Acetaminophen & $68.21 \%$ & 0.03 & 4.56 & $90.43 \%$ & 0.04 & 3.88 & $72.10 \%$ & 0.12 & \\
\hline Pronethalol & $40.91 \%$ & 0.06 & 14.01 & $105.51 \%$ & 0.07 & 6.53 & $45.57 \%$ & 0.05 & \\
\hline Propranolol & $36.81 \%$ & 0.07 & 19.95 & $138.69 \%$ & 0.06 & 4.10 & $42.14 \%$ & 0.03 & \\
\hline Diphenhydramine & $35.59 \%$ & 0.08 & 22.61 & $149.98 \%$ & 0.10 & 6.86 & $39.81 \%$ & 0.04 & \\
\hline Citalopram & $44.99 \%$ & 0.08 & 17.27 & $155.14 \%$ & 0.08 & 4.91 & $52.51 \%$ & 0.04 & \\
\hline Erythromycin & $37.82 \%$ & 0.08 & 20.56 & $131.26 \%$ & 0.03 & 2.62 & $48.35 \%$ & 0.05 & \\
\hline Carbamazepine & $67.95 \%$ & 0.04 & 6.02 & $109.12 \%$ & 0.04 & 3.93 & $68.14 \%$ & 0.03 & \\
\hline 10,11-DHC & $59.95 \%$ & 0.03 & 4.31 & $95.27 \%$ & 0.03 & 2.82 & $66.66 \%$ & 0.01 & \\
\hline Fluoxetine & $41.88 \%$ & 0.03 & 8.00 & $98.50 \%$ & 0.06 & 6.26 & $48.12 \%$ & 0.04 & \\
\hline Talopram & $46.49 \%$ & 0.05 & 10.04 & $161.16 \%$ & 0.07 & 4.60 & $56.13 \%$ & 0.03 & \\
\hline Loratadine & $57.38 \%$ & 0.02 & 2.90 & $105.35 \%$ & 0.07 & 7.04 & $64.40 \%$ & 0.03 & \\
\hline Diclofenac & $2.45 \%$ & 0.01 & 28.45 & $107.98 \%$ & 0.02 & 2.08 & $1.67 \%$ & 0.01 & \\
\hline Ibuprofen & $25.62 \%$ & 0.20 & 79.36 & $104.17 \%$ & 0.06 & 5.43 & $23.88 \%$ & 0.09 & \\
\hline
\end{tabular}




\section{Appendix 6.1}

The chromatograms for each of the biocides detected within the sample of sludgecake sampled during winter using the ZQ4000 mass spectrometer; a) BAC-C12 b) BAC-C14 c) BAC-C16 d) BAC-C18 e) DDMA f) HDTMA. The legend shows the $m / z$ of each compound and the signal intensity.

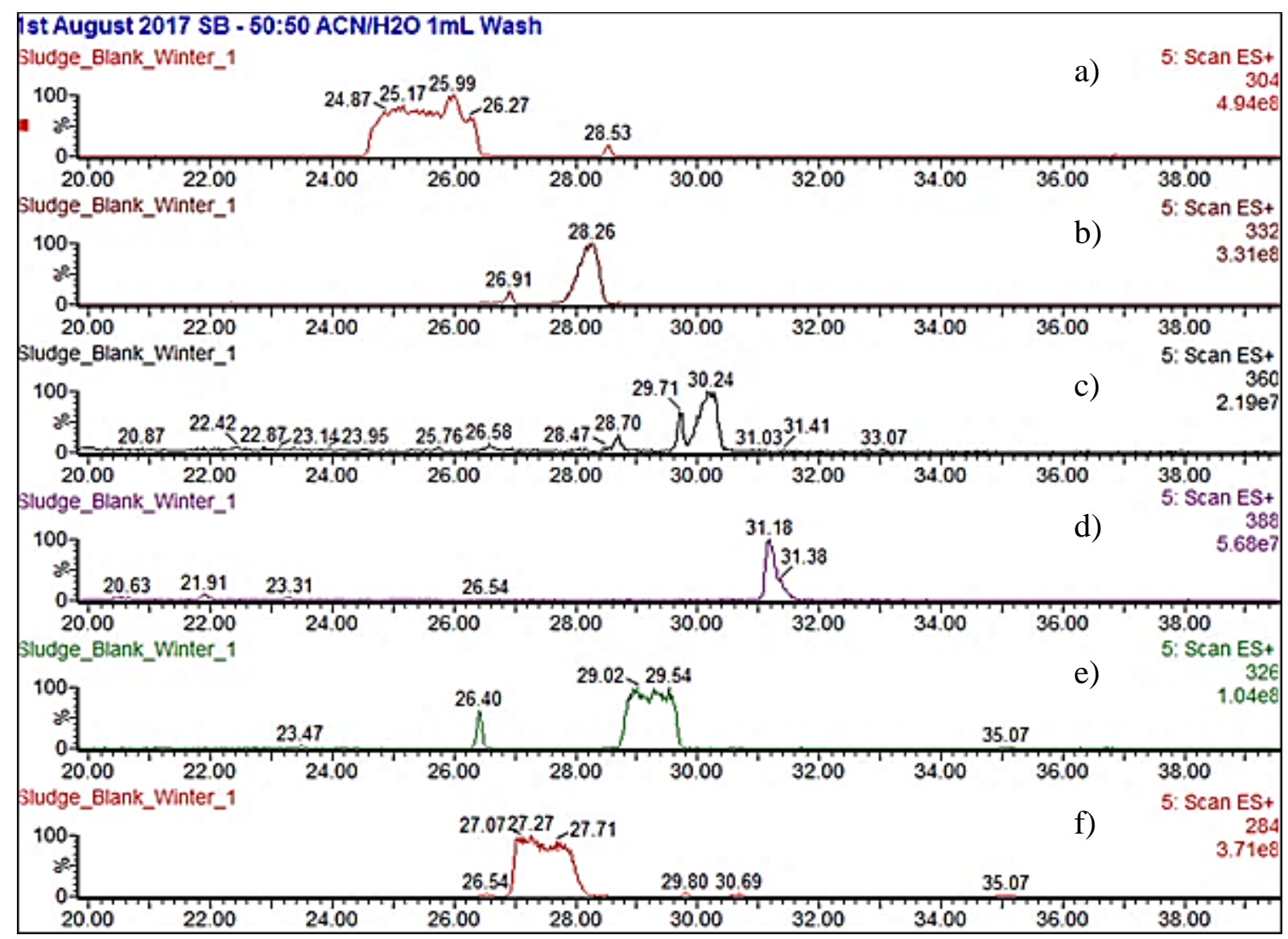


The chromatograms for each of the biocides detected within the 1:400 dilution of the sample of sludgecake sampled during winter using the LCQ mass spectrometer; a) BAC-C12 b) BAC-C14 c) BAC-C16 d) BAC-C18 e) DDMA f) HDTMA. The legend shows the $\mathrm{m} / \mathrm{z}$ of each compound and the signal intensity.

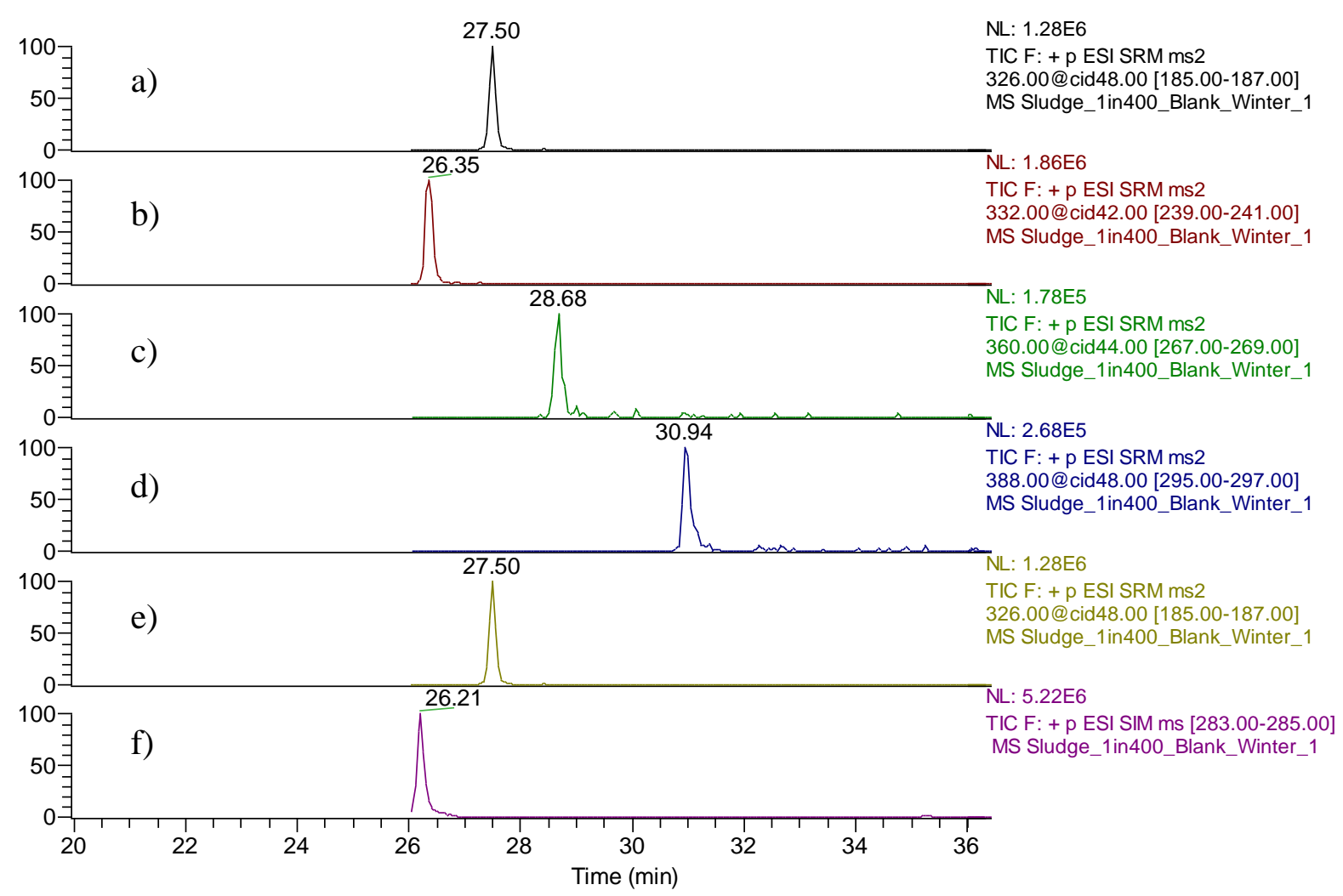




\section{Appendix 6.2}

The chromatograms for each of environmental matrices investigated using the LTQ Orbtitrap mass spectrometer showing the base peaks for; a) treated effluent b) winter sludgecake c) summer sludgecake d) wet biota e) lyophilised biota.

RT: 12.99 - 40.48

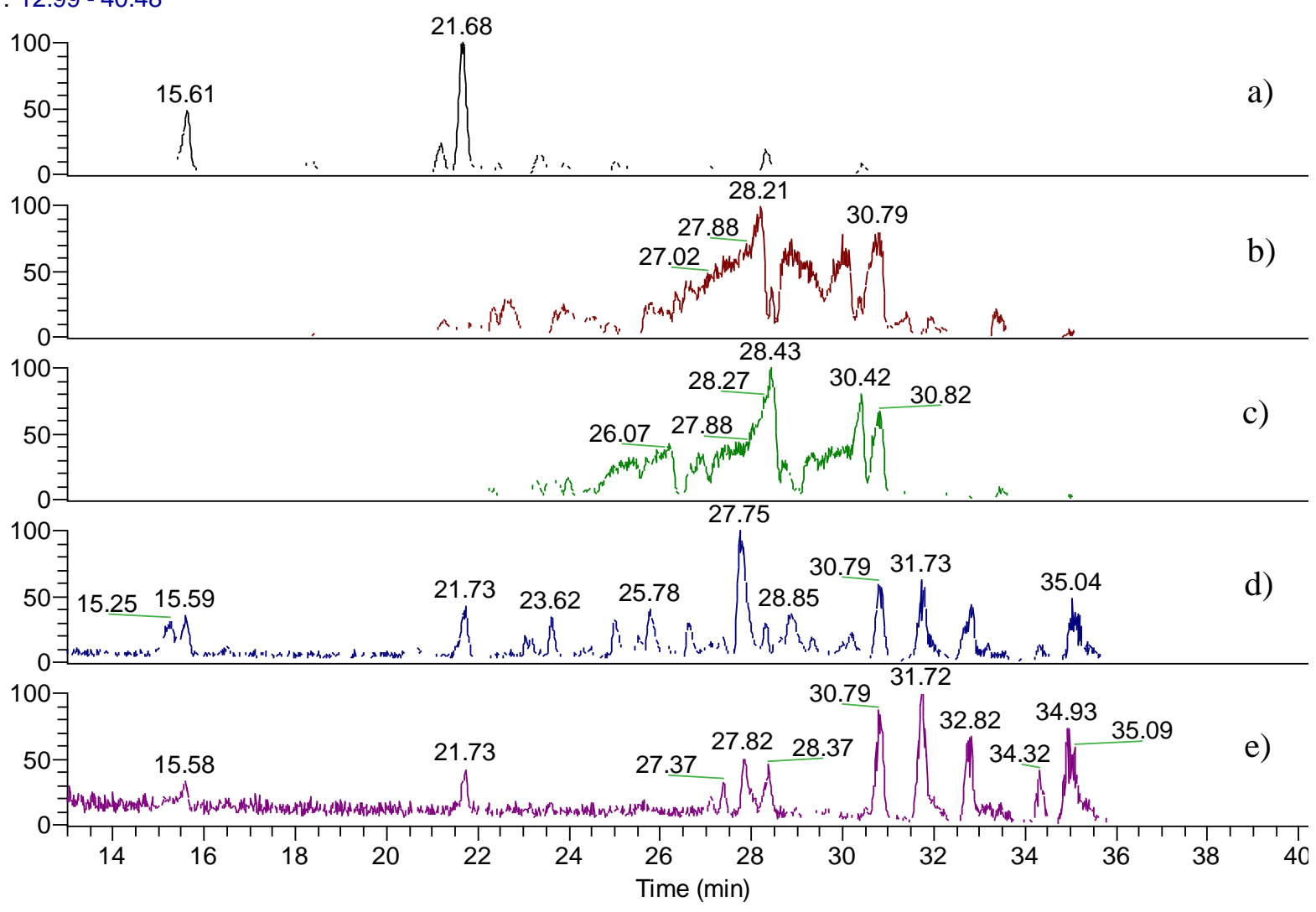

